\title{
Fouling Indices for Quantification of Natural Organic Matter Fouling and Cleaning in Ceramic and Polymeric Membrane Systems
}

\author{
by \\ Mohammad Alresheedi, B.A.Sc., M.A.Sc.
}

A thesis submitted to the Faculty of Graduate and Postdoctoral Affairs in partial fulfilment of the requirements for the degree of

Doctor of Philosophy
in

Environmental Engineering*

Department of Civil and Environmental Engineering

Carleton University

Ottawa, Ontario

Canada

*The Doctor of Philosophy in Environmental Engineering program is a joint program with the University of Ottawa administered by the Ottawa-Carleton Institute for Environmental Engineering

(C) 2019

Mohammad Alresheedi 


\begin{abstract}
$\underline{\text { Abstract }}$
Polymeric membranes have emerged as an economically effective treatment option to produce drinking water. More recently, ceramic membranes are raising interest in this field due to their unique physical properties, which may prove to be important in moving towards more robust and sustainable drinking water treatment methods. However, the loss of membrane permeability as a result of natural organic matter (NOM) fouling remains one of the biggest challenges for sustainable polymeric and ceramic membranes operation. A key challenge in membrane system is to understand how operating pressure and water temperature may impact fouling and subsequent cleaning in relationship to NOM. Further there is limited data to ascertain if ultrafiltration (UF) polymeric and ceramic systems will respond in similar or different manners to NOM fouling which then further impacts how respective systems need to be cleaned.
\end{abstract}

Fouling indices have been developed by means of simple, short, empirical filtration tests to assess the fouling potential of membrane feed water. The modified ultrafiltration fouling index (MFI-UF) is a standard test that is used to estimate a fouling index value that gives a general indication about the treatability of feed water or the need for pretreatment prior to a membrane unit. Unlike the MFI-UF, the unified membrane fouling index (UMFI) is used to quantify fouling a membrane is subjected to (i.e. reversible vs. irreversible), which provide different data on fouling. However, different approaches in fouling assessment may suggest that direct comparison lack context which lead to some disconnect between predicted and actual fouling in the field. Thus, the applicability of the MFI-UF to be effectively used in complement with the UMFI to predict NOM fouling under changes in 
filtration conditions needs to be examined with both ceramic and polymeric membranes systems.

In addition, due to the superior chemical resistance of ceramic membranes, the utilization of high cleaning $\mathrm{pH} 12$ solutions in a single, stepwise, or combined approach with sodium hypochlorite $(\mathrm{NaOCl})$ and sodium hydroxide $(\mathrm{NaOH})$ and ozone $\left(\mathrm{O}_{3}\right)$ clean in place $(\mathrm{CIP})$, which are not recommended with polymeric membranes, could highlight an advantage to ceramic membranes in drinking water applications for irreversible NOM fouling control.

The research showed that all NOM types exhibited higher MFI-UF values, and therefore, higher fouling propensity as pressure increased from 1 to 3 bars and water temperature decreased from $35^{\circ} \mathrm{C}$ to $5^{\circ} \mathrm{C}$ indicating the effect of pressure and temperature on the MFIUF fouling prediction. The NOM fouling potential order was consistent at different temperature which was the highest for the NOM mixture and proteins (BSA) followed by alginate and lastly humic acid. The MFI-UF normalization model was useful in estimating the fouling potential away from standard testing conditions $\left(2\right.$ bar and $\left.20^{\circ} \mathrm{C}\right)$. Therefore, MFI-UF values measured at standard testing conditions can be altered to actual filtration conditions by adjusting for the pressure and viscosity terms in the MFI-UF equation.

While the MFI-UF was impacted by operational conditions, variation in feed water temperature in polymeric and ceramic membrane systems demonstrated negative impacts on NOM fouling and cleaning. NOM fouling increased as water temperature decreased from $20^{\circ} \mathrm{C}$ to $5^{\circ} \mathrm{C}$ while fouling decreased as temperature increased from $20^{\circ} \mathrm{C}$ to $35^{\circ} \mathrm{C}$. 
The UMFI analysis showed that irreversible NOM fouling ratios increased at cold water condition $\left(5^{\circ} \mathrm{C}\right)$, along with decreased in backwash and chemical cleaning effectiveness of both membrane types. The UMFI results obtained in the polymeric and ceramic UF systems demonstrated useful fit with the MFI-UF prediction for establishing NOM fouling trend and order with temperature. Therefore, utilizing the MFI-UF is useful to monitor changes in NOM fouling behavior with temperature to achieve long-term operational sustainability.

Under an equivalent fouling and cleaning conditions, NOM fouling order of a ceramic UF membrane was found to be similar to their polymeric counterparts in the following order: the NOM mixture $>$ BSA $>$ alginate $+\mathrm{Ca}^{+2}>$ alginate- $-\mathrm{Ca}^{+2}>$ humic acid, suggesting that both membranes are susceptible to fouling by proteins. Ceramic membranes, however, demonstrated better performance in terms of backwashing, thus, lower irreversible fouling highlighting the ease of integration of ceramic membranes into drinking water applications. Chemical cleaning of a ceramic UF membrane using $\mathrm{O}_{3}$ CIP for 1 hour at a ratio of 0.50 $\mathrm{mgO}_{3} / \mathrm{mgC}$ demonstrated higher reduction of irreversible fouling of hydrophobic (humic acid) and hydrophilic (alginate) NOM fractions and lower sensitivity to water quality conditions compared to 4 hours cleaning using single, stepwise, or combined use of $\mathrm{NaOCl}$ and $\mathrm{NaOH} \mathrm{CIP}$. Since the ceramic membrane is quite robust, it allows for some creativity regarding fouling mitigation techniques, particularly backwashes and chemical cleaning. 


\section{Acknowledgements}

I wholeheartedly want to express my gratitude to my supervisor Dr. Onita Basu. Her support and understanding, both academically and personally, have made this long journey less arduous and more rewarding. Her support from start to finish, encouragement and exceptional guidance throughout this research project has been a constant source of motivation.

I wish to express my deep and sincere gratitude to Dr. Benoit Barbeau for his guidance and contribution to this research as well as providing me with excellent laboratories for conducting my research. My deepest appreciation goes to Mireille Blais and all the technicians at the CREDEAU laboratories at Polytechnique, Montréal, for their excellent assistance. There are too many names to list but a huge thank you to everyone in the Dr. Barbeau research group both past and present.

I also would like to thank all the faculty and staff in the Department of Civil and Environmental Engineering at Carleton University, especially Dr. Marie Tudoret, for her constant availability to provide help and advice in the laboratory. I am very grateful to all my colleagues in the Dr. Basu research group for their cooperative manner and wonderful support.

I give special thanks to Geoff Seatter and Kerwin Lewis for their help with installation of the automated membrane filtration system. Their patience and countless efforts to explain 
the operation of solenoid valves, resistors, arduino board, etc., are much appreciated. Thank you.

I would like to thank summer students, Bia Pereira and Marina Guimarães for their assistance during portions of the experiments.

I would like to express my appreciation for the love and support of my family and friends. Their unconditional understanding and encouragement during this research project have motivated me to keep fighting but without struggling.

I would also like to acknowledge and thank all the members of my thesis defense committee. Thank you for your time and contributions.

This research was funded by the Saudi Arabia Ministry of Education (MOE) and Natural Sciences and Engineering Research Council of Canada (NSERC). 


\section{Table of Contents}

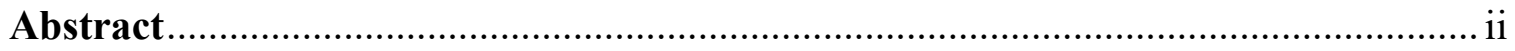

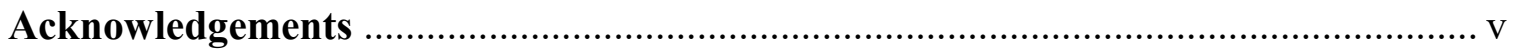

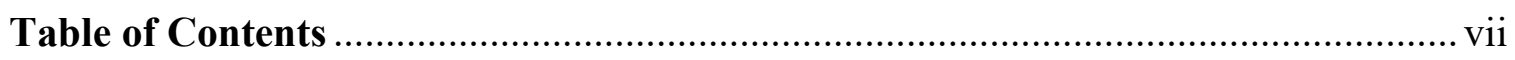

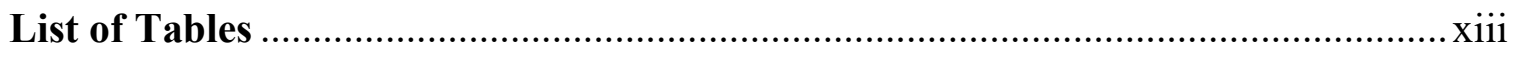

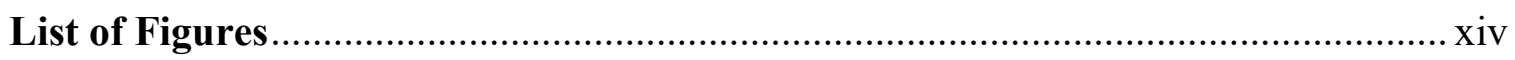

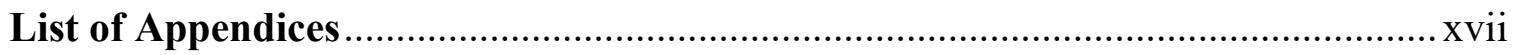

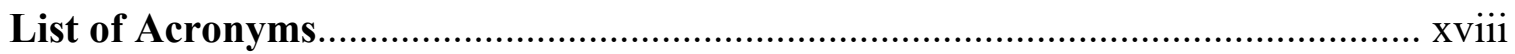

Chapter 1- Introduction and Research Objectives ............................................... 1

1.1 Statement of Problem............................................................................. 1

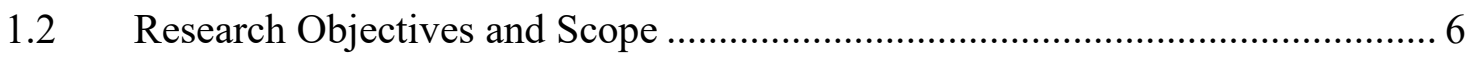

1.3 Thesis Structure...................................................

1.4 Articles Summary and Authors Contribution............................ 10

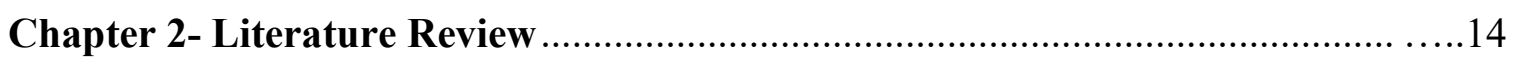

$2.1 \quad$ Natural Organic Matter (NOM) .................................................................. 15

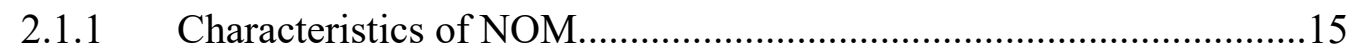

2.1.2 Measurements of NOM in Water................................ 17

2.2 Membrane Filtration Processes for Drinking Water Treatment....................... 19

2.3 Membrane Fouling: Source, Mechanism, and Assessment..................22

2.3.1 Fouling Source......................................... 22

2.3.2 Fouling Mechanism...................................... 23

2.3.3 Fouling Assessment.........................................24

2.3.3.1 Resistance in Series (RIS)........................24

2.3.3.2 Unified Membrane Fouling Index (UMFI).............25

2.3.3.3 Fouling Indices.................................26

2.3.3.3.1 Silt Density Index (SDI) ..................27

2.3.3.3.2 Modified Fouling Index $\left(\mathrm{MFI}_{0.45}\right) \ldots \ldots \ldots . . .29$

2.3.3.3.3 Modified Ultrafiltration Fouling Index (MFI-UF)

2.4 Water Contaminants Removal Using Polymeric and Ceramic Membranes ....33

2.4.1 Polymeric Membranes Applications in Drinking Water Treatment....33 
2.4.2 Ceramic Membranes Applications in Drinking Water Treatment.......35

2.5 Effect of Water Temperature on Membrane Filtration Performance............39

2.6 Chemical Cleaning of Polymeric and Ceramic Membranes..................40

2.6.1 Polymeric Membranes Cleaning..............................42

2.6.2 Ceramic Membranes Cleaning..................................44

2.7 Summary of Research Needs.....................................................46

Chapter 3- Application of MFI-UF Fouling Index with NOM Fouling under Various

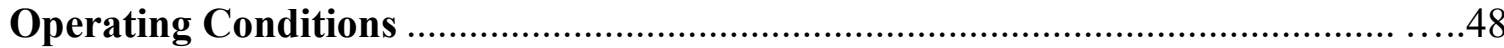

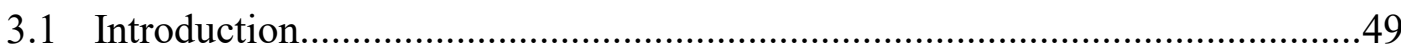

3.2 Materials and Methods................................................ 51

3.2.1 Experimental Setup and Approach...............................51

3.2.2 Feed Solutions...........................................54

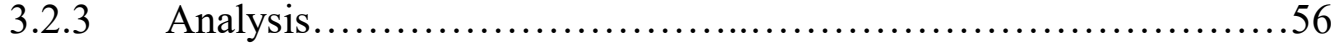

3.2.3.1 Molecular Weight Fractionation of NOM...............56

3.2.3.2 MFI-UF Data Analysis...........................57

3.2.3.3 Modelling of the MFI-UF.............................58

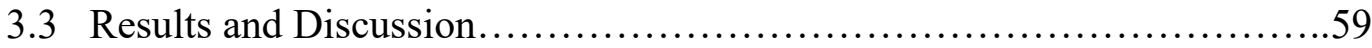

3.3.1 Molecular Weight Distribution of Feed Solutions..................59

3.3.2 Effect of Variation of Operating Pressure and Water Temperature on the MF-UF Measurements to Predict NOM Fouling...........................60

3.3.2.1 MFI-UF Measurements under Variable Operating Pressure .60

3.3.2.2 MFI-UF Measurements under Variable Water Temperature

3.3.2.3 MFI-UF Surface Plots and TOC Rejection................66

3.3.3 Modelling of MFI-UF (MFI-UF $F_{\text {exp }}, M^{2}$ MFI $_{\text {nor, }}$ MFI-UF $F_{\text {pr }}$ )..........69

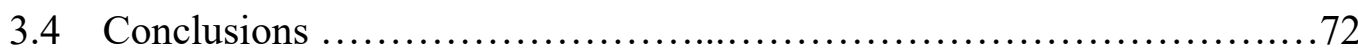

3.5 Acknowledgements ......................................... 73

3.6 References.................................................... 73

Chapter 4- Investigation into the Temperature Effect on NOM Fouling andCleaning in Submerged Polymeric Membrane Systems................................... 78

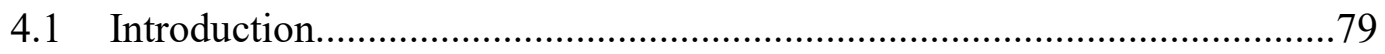


4.2 Materials and Methods.................................................... 81

4.2.1 Feed Solutions.................................................. 81

4.2.2 Experimental Setup and Approach............................83

4.2.2.1 Submerged Polymeric Membrane Setup.................83

4.2.2.2 Fouling Resistances using the Unified Membrane Fouling Index (UMFI) Method …............................... 85

4.2.2.3 Specific Cake Resistance...............................87

4.2.2.4 MFI-UF Fouling Index.................................88

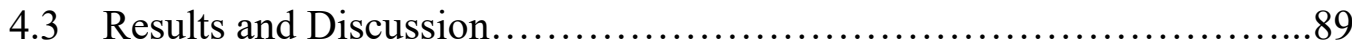

4.3.1 Effect of Water Temperature Condition on NOM Fouling ..........89

4.3.2 Fouling Indices Assessment at Different Water Temperature

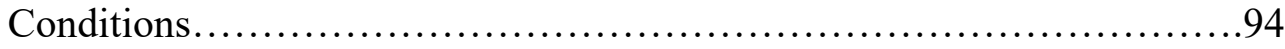

4.3.2.1 Reversible and Irreversible Fouling Indices...............94

4.3.2.2 Correlation of UMFI and MFI-UF Indices..................98

4.3.3 Impact of Water Temperature on Chemical Cleaning ..................99

4.4 Conclusions ...................................................... 102

4.5 Acknowledgements ................................................ 103

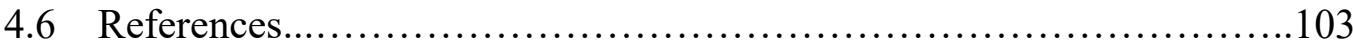

\section{Chapter 5- Effects of Feed Water Temperature on Irreversible Fouling of Ceramic}

Ultrafiltration Membranes.........................................................................109

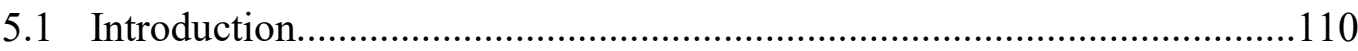

5.2 Materials and Methods.............................................113

5.2.1 Feed Water Solution........................................ 113

5.2.2 Ceramic UF Fouling and Cleaning Experiments.................114

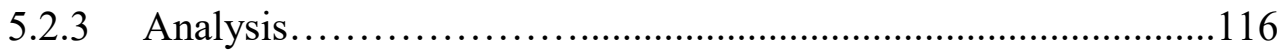

5.2.3.1 The Unified Membrane Fouling Index (UMFI)..........116

5.2.3.2 Specific Cake Resistance.............................117

5.2.3.3 Carbon and Fluorescence Excitation and Emission Matrix (FEEM) Analyses.....................................................118

5.2.3.4 MFI-UF Fouling Index.............................120

5.2.3.5 Statistical Analysis....................................121

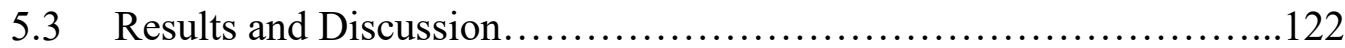

5.3.1 Effect of Feed Water Temperature on Ceramic UF Fouling ...122 
5.3.2 Impact of Feed Water Temperature on Backwash and Chemical Cleaning Effectiveness............................................126

5.3.3 Suitability of the MFI-UF Index for Fouling Prediction with Ceramic Membranes...................................................131

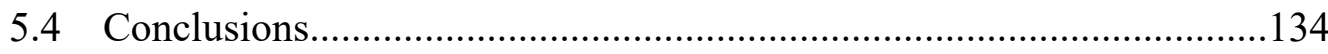

5.5 Acknowledgements............................................. 136

5.6 References......................................................

\section{Chapter 6- Comparisons of NOM Fouling and Cleaning of Ceramic and Polymeric}

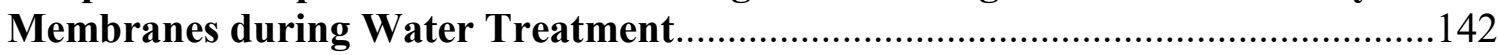

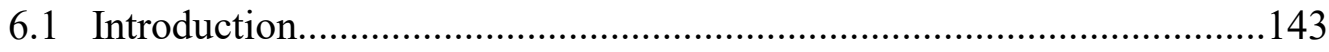

6.2 Materials and Methods.................................................146

6.2.1 Model Foulants................................................146

6.2.2 Fouling Experiments.....................................................

6.2.3 Chemical Cleaning Experiments...................................150

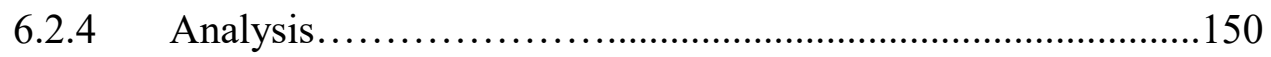

6.2.4.1 Fouling Resistances and Specific Flux Recovery....150

6.2.4.2 Carbon Mass Balance................................151

6.2.4.3 Fluorescence Excitation and Emission (FEEM)......152

6.3 Results and Discussion..............................................

6.3.1 Ceramic versus Polymeric UF Performance Comparison.......153

6.3.1.1 NOM Fouling and Removal by Ceramic and Polymeric UF Membranes ............................................... 153

6.3.1.2 Hydraulic BW Efficacy for Ceramic and Polymeric UF Membranes............................................... 157

6.3.1.3 Fouling Analysis Based on a Carbon Mass

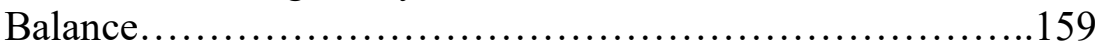

6.3.1.4 FEEM Analysis of Permeate and Backwash Waters.................................................. 160

6.3.2 Ceramic versus Polymeric UF Cleaning under Equivalent

Cleaning Protocols

6.3.2.1 Fouling Resistance Removal and Specific Flux Recovery.

6.3.2.2 Chemically Reversible and Irreversible Carbon Mass Balance in Wash Waters.......................................163

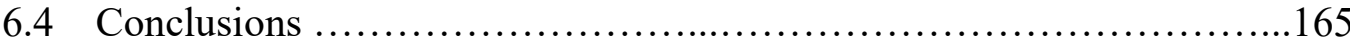




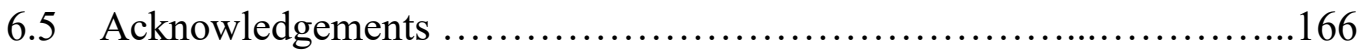

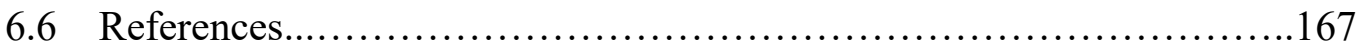

\section{Chapter 7- Chemical Cleaning of Ceramic Ultrafiltration Membranes - Ozone versus Conventional Cleaning Chemicals.}

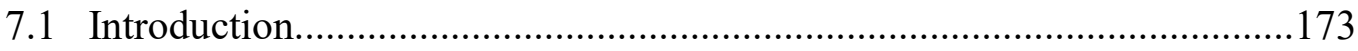

7.2 Materials and Methods.............................................. 176

7.2.1 Model Foulants................................................ 176

7.2.2 Experimental Setup and Approach.................................177

7.2.3 Chemical Cleaning Experiments....................................179

7.2.3.1 Cleaning $\mathrm{pH}$ Effect (pH 11 vs. $\mathrm{pH} 12)$..................179

7.2.3.2 Chemicals Cleaning Sequence $(\mathrm{NaOCl} / \mathrm{NaOH}$,

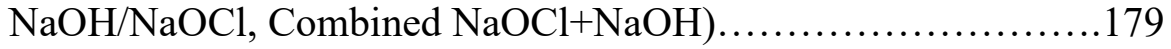

7.2.3.3 Ceramic UF Cleaning using $\mathrm{O}_{3}$ CIP...........................180

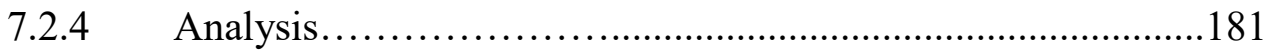

7.2.4.1 The Unified Membrane Fouling Index (UMFI) .......181

7.2.4.2 Carbon Mass Balance..................................182

7.2.4.3 Surface Tension $(\lambda)$ Measurements..................182

7.3 Results and Discussion............................................... 183

7.3.1 Ceramic UF Fouling by Alginate and Humic Acids...............183

7.3.2 Ceramic UF Cleaning: Effect of Cleaning $\mathrm{pH}$ and Cleaning Agent

7.3.3 Ceramic UF Cleaning Sequence: combined use of $\mathrm{NaOCl}$ and

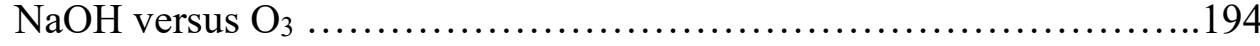

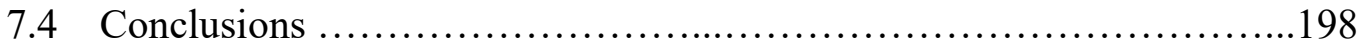

7.5 Acknowledgements ................................................... 198

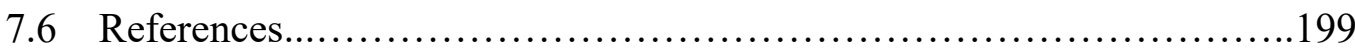

Chapter 8- Summary, Conclusions and Recommendations.......................205

8.1 Summary of Findings and Conclusions ...................................................205

8.2 Overall Significant Conclusions and Contributions.....................208

8.3 Recommendations for Future Work................................210

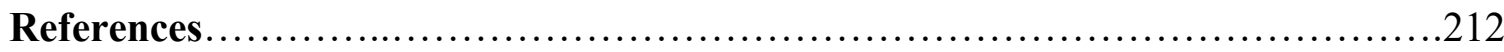




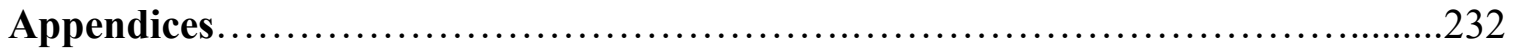

Appendix (A) - Experimental Methods...............................2232

Appendix (B) - MFI-UF Statistical Analysis Summary......................240

Appendix (C) - Macro Filter Code used in Fouling Data Analysis............243

Appendix (D) - Randomized Block Design (RBD) (SPSS Output)...........245 


\section{List of Tables}

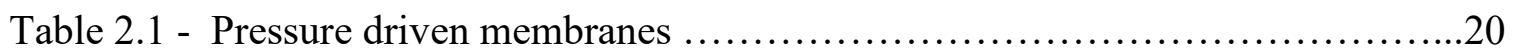

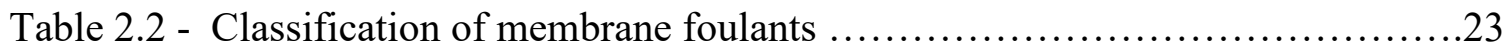

Table 2.3 - Classification of various cleaning chemicals ...........................41

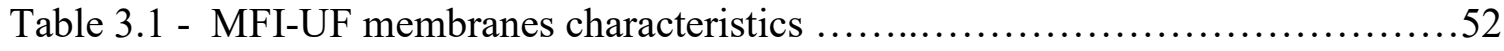

Table 4.1 - Molecular weight fractionation and zeta potential of feed solutions..........83

Table 4.2 - Operational conditions of filtration experiments $\ldots \ldots \ldots \ldots \ldots \ldots \ldots \ldots \ldots \ldots . .84$

Table 4.3 - MFI-UF membrane characteristics ..................................... 89

Table 4.4 - MFI-UF index prediction of NOM fouling at different temperatures..........98

Table 5.1 - Estimated specific cake resistance and NOM retention values at different

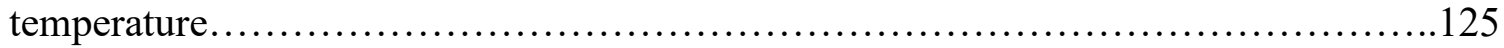

Table 6.1 - Molecular weight fractionation and zeta potential of feed solutions.........147

Table 6.2 - Ceramic and polymeric membranes characteristics......................149

Table 6.3 - NOM removals by the ceramic and polymeric UF ......................156

Table $6.4-\mathrm{R}_{\mathrm{hr}}$ and $\mathrm{R}_{\text {hir }}$ slope values of different feed NOM solutions .................157

Table 7.1 - Hydraulically reversible and irreversible carbon percentages...............186

Table 7.2 - Average Surface tension values of CIP water ...............................191

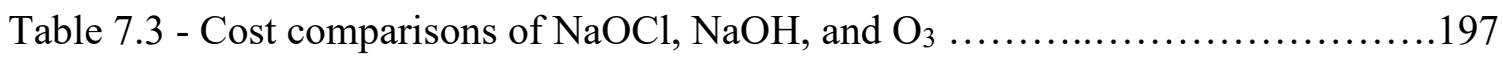




\section{List of Figures}

Figure 2.1 - Illustration of membrane fouling mechanisms (blocking models)............24

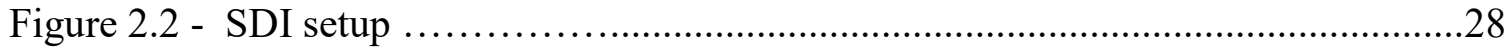

Figure 2.3 - Ratio of filtration time and filtrate volume $(\mathrm{t} / \mathrm{V})$ as a function of filtrate

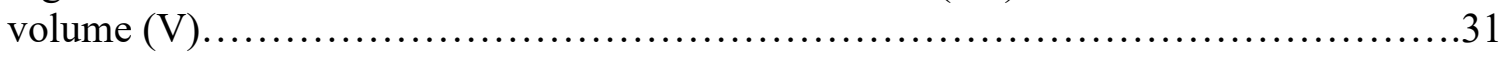

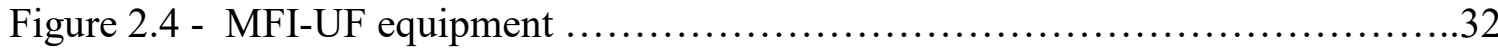

Figure 3.1 - A schematic representation of the MFI-UF setup .......................54

Figure 3.2 - Molecular weight distribution of feed water solutions ..................60

Figure 3.3 - Example of MFI-UF and filtration mechanism graphs for the NOM fractions at different pressure $\left(\mathrm{T}=20{ }^{\circ} \mathrm{C}\right)$; (A)MFI-UF vs time $(\mathrm{P}=1 \mathrm{bar}) ;(\mathrm{B}) \mathrm{d}^{2} \mathrm{t} / \mathrm{dV}^{2}$ vs dt/dV curves $\left(\mathrm{P}=1\right.$ bar); (C)MFI-UF vs time ( $\mathrm{P}=3$ bar); (D) $\mathrm{d}^{2} \mathrm{t} / \mathrm{dV}^{2} \mathrm{vs} d \mathrm{dt} / \mathrm{dV}$ curves $(\mathrm{P}=3$ bar).......

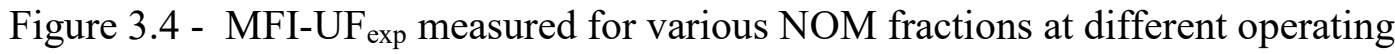
pressure $\left(\mathrm{T}=20{ }^{\circ} \mathrm{C}\right)$.

Figure 3.5 - Example of MFI-UF and filtration mechanism graphs for the NOM fractions at different temperature ( $\mathrm{P}=1 \mathrm{bar}) ;(\mathrm{A}) \mathrm{MFI}-\mathrm{UF}$ vs time $\left(\mathrm{T}=5^{\circ} \mathrm{C}\right) ;(\mathrm{B}) \mathrm{d}^{2} \mathrm{t} / \mathrm{dV}^{2} \mathrm{vs} \mathrm{dt} / \mathrm{dV}$ curves $\left(\mathrm{T}=5^{\circ} \mathrm{C}\right)$; (C) MFI-UF vs time $\left(\mathrm{T}=35^{\circ} \mathrm{C}\right) ;(\mathrm{D}) \mathrm{d}^{2} \mathrm{t} / \mathrm{dV}^{2} \mathrm{vs} \mathrm{dt} / \mathrm{dV}$ curves $(\mathrm{T}=35$ $\left.{ }^{\circ} \mathrm{C}\right)$

Figure 3.6 - MFI-UF exp measured for various NOM fractions at different water temperature $(\mathrm{P}=2$ bar $)$.

Figure 3.7 - 3D surface plots of the MFI-UF as a function of pressure and temperature.68

Figure 3.8 - MFI-UF and TOC rejection with water temperature conditions ( $\mathrm{P}=2$ bar).69

Figure 3.9 - MFI-UF as a function of filtered water volume for the 4 different synthetic solutions (A) Humic Acid, (B) BSA, (C) Sodium Alginate and (D) NOM Mixture......70

Figure 3.10 - MFI-UF exp, vs. MFI-UF nor vs. MFI-UF pr $_{\text {for }}$ different model solutions......72

Figure 4.1 - A schematic representation of the bench scale polymeric membrane setups

Figure 4.2 - Estimation of fouling resistances using the UMFI method . .86

Figure 4.3 - NOM Fouling graphs (A) Humic Acid; (B) BSA; (C) Sodium Alginate; (D)

Mixture.

Figure 4.4 - (A) Changes in specific cake resistance with temperature; (B) estimated

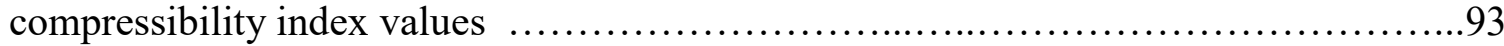

Figure 4.5 - SEM images of NOM fouling layers at $5^{\circ} \mathrm{C}$ and $20^{\circ} \mathrm{C}(\mathrm{P}=1$ bar $)$ using $0.45 \mu \mathrm{m}$ membrane filters. 
Figure 4.6 - Estimated UMFI values at different temperature conditions. .96

Figure 4.7 - Hydraulically irreversible to reversible fouling index ratios at different water temperature conditions.

Figure 4.8- Relationship between UMFI fouling resistances and MFI-UF fouling index.99 Figure 4.9 - Chemical cleaning efficiency at different filtration water temperature.

(A) $\mathrm{UMFI}_{\mathrm{cr}} / \mathrm{UMFI}_{\text {hir }}$ ratios; (B) Specific flux recovery

Figure 5.1- A schematic representation of the ceramic UF setup.

Figure 5.2- Changes in the UMFI fouling index at different water temperature.

(A) $\mathrm{UMFI}_{\mathrm{f}}$ (exp): UMFI before viscosity correction; (B) UMFI (nor): UMFI after viscosity correction

Figure 5.3- Changes in $\mathrm{UMFI}_{\mathrm{f}}$ values due to changes in viscosity versus fouling with temperature

Figure 5.4- FEEM images of the ceramic UF permeate of NOM mixture at 5 and $20^{\circ} \mathrm{C}$

Figure 5.5 - UMFI fouling indices at different water temperature conditions

Figure 5.6 - Effect of water temperature on backwash effeciency.

Figure 5.7 - FEEM images of the ceramic UF backwash water of NOM mixture at 5 and $20^{\circ} \mathrm{C}$.

Figure 5.8 - Effect of water temperature on chemical cleaning effeciency. 130

Figure 5.9 - FEEM images of the ceramic UF $\mathrm{NaOCl}$ wash water of NOM mixture at 5 and $20{ }^{\circ} \mathrm{C}$

Figure 5.10 - (A) MFI-UF fouling index values at different temperature; (B) Changes in specific cake resistance with temperature; (C) NOM retention by the MFI-UF membrane at different temperature.

Figure 5.11 - (A) Relationship between $\mathrm{UMFI}_{\mathrm{f}}$ and MFI-UF fouling indices; (B) Specific cake resistance with temperature; (C) NOM retention by MFI-UF and ceramic UF membranes.

Figure 6.1 - Automated membrane system .................................. 148

Figure 6.2 - Resistances estimation using the slopes fouling graphs

Figure 6.3 - (A) Ceramic UF fouling by different NOM solutions (Flux: 100 LMH, BW: every 4 hours for 20s at $30 \mathrm{psi}$ ); (B) Polymeric UF fouling by different NOM solutions (Flux: $100 \mathrm{LMH}, \mathrm{BW}$ : every 4 hours for $20 \mathrm{~s}$ at $30 \mathrm{psi}$ )..... 154

Figure 6.4 - Backwash efficiency ( $\mathrm{R}_{\mathrm{hr}} \mathrm{R}_{\mathrm{f}}$ ratios). (A) Ceramic UF; (B) Polymeric UF. Higher ratios indicates higher BW efficiency and lower irreversibility... 158 
Figure 6.5 - Mass balance of carbon (expressed as \% of the feed) found as hydraulically reversible (in the BW water), hydraulically irreversible (left on the membrane) or in the

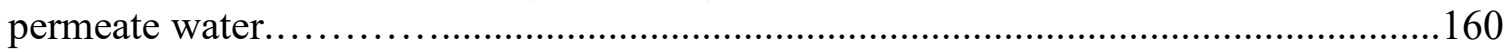

Figure 6.6 - FEEM plots of NOM mixture (permeate and backwash water)............161

Figure 6.7 - Cleaning efficiency based on the removal of chemically reversible resistance $\left(\mathrm{R}_{\mathrm{cr}}\right)$ - $\mathrm{pH} 11 ; 35^{\circ} \mathrm{C}$. (A) Ceramic UF; (B) Polymeric UF.......................... 162

Figure 6.8 - Chemically reversible and irreversible \%carbon $\left(\mathrm{pH} 11 ; 35^{\circ} \mathrm{C}\right) \ldots \ldots \ldots . .164$

Figure 6.9 - FEEM plots of $\mathrm{NOM}$ mixture $(\mathrm{NaOCl}$ and $\mathrm{NaOH}$ wash water $) .. . \ldots \ldots \ldots . . .165$

Figure 7.1 - Automated ceramic membrane system.............................178

Figure 7.2 - Ceramic UF membrane fouling by different NOM solutions (Constant Flux: $100 \mathrm{LMH}$, BW: every 4 hours $\left(0.4 \mathrm{~m}^{3} / \mathrm{m}^{2}\right)$ for $20 \mathrm{~s}$ at 30 psi. $\mathrm{pH}=7.5$. UMFI: Unified

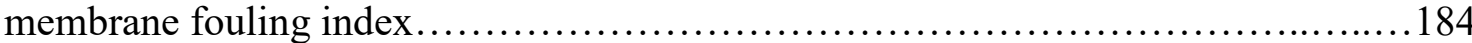

Figure 7.3 - Hydraulically reversible fouling index (UMFI $\left.{ }_{h r}\right)$ and hydraulically irreversible fouling index $\left(\mathrm{UMFI}_{h i r}\right)$ ratios 185

Figure 7.4 - Effect of cleaning $\mathrm{pH}$ and cleaning agent on the removal of $\mathrm{UMFI}_{\mathrm{cr}}$ of ceramic UF

Figure 7.5 - Correlations between chemically reversible fouling index recovery $\left(\mathrm{UMFI}_{\mathrm{cr}}\right)$ and $\%$ carbon removed by various chemical cleaning: (A) $\mathrm{NaOCl}$ CIP vs. $\mathrm{O}_{3} \mathrm{CIP}$; (B) $\mathrm{NaOH}$ CIP vs. $\mathrm{O}_{3}$ CIP. Ozone CIP at a ratio of $0.50 \mathrm{mg} \mathrm{O} / \mathrm{mg} \mathrm{C}$.

Figure 7.6 - (A) Cumulative $\mathrm{O}_{3}$ demand versus cleaning time; (B) Cumulative carbon removed versus cleaning time. $\mathrm{O}_{3} \mathrm{CIP}$ at a ratio of $0.50 \mathrm{mg} \mathrm{O} 3 / \mathrm{mg} \mathrm{C}$

Figure 7.7 - Recovery of UMFIcr following various ceramic UF cleaning sequence. (A) Alginate $+\mathrm{Ca}^{+2}$; (B) Alginate-Ca ${ }^{+2}$ (C) Humic acids 196 


\section{List of Appendices}

Appendix (A) - Experimental Methods.....................................2232

Appendix (B) - MFI-UF Statistical Analysis Summary..........................240

Appendix (C) - Macro Filter Code used in Fouling Data Analysis................243

Appendix (D) - Randomized Block Design (RBD) (SPSS Output)..............245 


\section{List of Acronyms}

\begin{tabular}{|c|c|}
\hline Alginate - $\mathbf{C a}^{+2}$ & Alginate without calcium \\
\hline Alginate $+\mathbf{C a}^{+2}$ & Alginate with calcium \\
\hline ANOVA & Analysis of variance \\
\hline ASTM & American Society for Testing and Materials \\
\hline BAC & Biological activated carbon \\
\hline BDOC & Biodegradable dissolved organic carbon \\
\hline BSA & Bovine serum albumin \\
\hline CA & Cellulose acetate \\
\hline CEB & Chemical enhanced backwash \\
\hline CIP & Clean in place \\
\hline CRBD & Completely randomized block design \\
\hline CWF & Clean water flux \\
\hline DBPs & Disinfection by-products \\
\hline DI & Deionized water \\
\hline DOC & Dissolved organic carbon \\
\hline EDTA & Ethylenediaminetetraacetic acid \\
\hline EPA & Environmental Protection Agency \\
\hline EPS & Extracellular polymer substances \\
\hline FEEM & Fluorescence excitation and emission matrix \\
\hline GLM & Generalized linear model \\
\hline HAA & Haloacetic acids \\
\hline $\mathbf{H C l}$ & Hydrochloric acid \\
\hline ID & Inside diameter \\
\hline kDa & Kilodaltons \\
\hline LMH & Liter per membrane area per hour \\
\hline LPM & Liter per minute \\
\hline MBR & Membrane bio reactor \\
\hline MF & Microfiltration \\
\hline
\end{tabular}




\begin{tabular}{|c|c|}
\hline MFI 0.45 & Modified fouling index \\
\hline MFI-UF & Modified ultrafiltration fouling index \\
\hline MFI-UF $\exp$ & Experimental modified ultrafiltration fouling index \\
\hline MFI-UF nor & Normalized modified ultrafiltration fouling index \\
\hline MFI-UFpr & Predicted modified ultrafiltration fouling index \\
\hline MGD & Million gallon per day \\
\hline MW & Molecular weight \\
\hline MWCOs & Molecular weight cut offs \\
\hline $\mathrm{NaOCl}$ & Sodium hypochlorite \\
\hline $\mathrm{NaOCl} / \mathrm{NaOH}$ & $\mathrm{NaOCl}$ followed by $\mathrm{NaOH}$ \\
\hline $\mathrm{NaOCl}+\mathrm{NaOH}$ & Combined $\mathrm{NaOCl}$ and $\mathrm{NaOH}$ \\
\hline $\mathrm{NaOH}$ & Sodium hydroxide \\
\hline $\mathrm{NaOH} / \mathrm{NaOCl}$ & $\mathrm{NaOH}$ followed by $\mathrm{NaOCl}$ \\
\hline $\mathbf{N F}$ & Nanofiltration \\
\hline NOM & Natural organic matter \\
\hline NTU & Nephelometric turbidity unit \\
\hline $\mathbf{O}_{3}$ & Ozone \\
\hline OD & Outside diameter \\
\hline PAN & Polyacrylonitrile \\
\hline PES & Polyethersulfone \\
\hline POC & Particulate organic carbon \\
\hline PS & Polysulfone \\
\hline PVDF & Polyvinylidene fluoride \\
\hline $\mathbf{R}_{\mathbf{c}}$ & Cake layer resistance \\
\hline Reir & Chemically irreversible fouling resistance \\
\hline $\mathbf{R}_{\text {cr }}$ & Chemically reversible fouling resistance \\
\hline $\mathbf{R}_{\mathbf{f}}$ & Total fouling resistance \\
\hline $\mathbf{R}_{\text {hir }}$ & Hydraulically irreversible fouling resistance \\
\hline $\mathbf{R}_{\mathbf{h r}}$ & Hydraulically reversible fouling resistance \\
\hline RIS & Resistance in series \\
\hline
\end{tabular}




\begin{tabular}{|c|c|}
\hline $\mathbf{R}_{\mathbf{m}}$ & Intrinsic membrane resistance \\
\hline RO & Reverse osmosis \\
\hline SDI & Silt density index \\
\hline SEM & Sodium dodecyl sulfate \\
\hline SMPs & Soluble microbial products \\
\hline SUVA & Specific ultraviolet absorbance \\
\hline THM & Trihalomethane \\
\hline TMP & Transmembrane pressure \\
\hline TOC & Total organic carbon \\
\hline UF & Ultrafiltration \\
\hline UMFI & Unified membrane fouling index \\
\hline UMFI & Chemirally irreversible fouling index \\
\hline UMFI & Chemically reversible fouling index \\
\hline UMFI & Total unified fouling index \\
\hline UMFI & Hydraulically irreversible fouling index \\
\hline UMFI & Hydraulically reversible fouling index \\
\hline UVA254 & Ultraviolet absorbance \\
\hline Vs & Specific filtered water volume \\
\hline
\end{tabular}




\section{Chapter 1}

\section{Introduction and Research Objectives}

\subsection{Statement of problem}

The application of polymeric membranes has gained wide acceptance as an effective technology in drinking water treatment. More recently, ceramic membranes are raising interest in this field due to their unique physical properties, which may prove to be important in moving towards more robust and sustainable drinking water treatment methods. The main advantages of ceramic membranes over polymeric membranes lies in their superior mechanical, thermal, and chemical stability, which make them ideal for industrial applications that deal with challenging water conditions such as in food and beverage production (Vasanth et al., 2013; Zhou et al., 2010). These characteristics can also prove valuable in drinking water treatment applications due to the higher fluxes and backwash pressures that can be applied, and robustness against aggressive chemical cleaning conditions. Unfortunately, a current major limitation preventing their widespread use over polymeric membranes in drinking water treatment is cost. However, the cost of production due to technological advances has started to decrease and their higher capital cost may be compensated by lower operating costs due to higher permeabilities and longer lifetimes (Lee et al., 2014; Freeman and Shorney-Darby, 2011; Lehman and Liu, 2009). As a result, ceramic membranes are becoming cost-competitive for full-scale operations and may potentially become the more economical alternative to polymeric membranes.

Fouling is the major challenge for successful implementation of both polymeric and ceramic membranes in water filtration. Fouling can decrease membrane separation, 
increase operating costs, and ultimately decreases the lifetime of the membrane. Previous studies on membrane fouling have provided valuable insights on the major foulants present during the filtration of natural waters. This includes natural organic matter (NOM), inorganic substances, particulate/colloids matter, and microbiological compounds (Guo et al., 2012; Zularisam et al., 2006). Among these foulants, fouling by NOM such as humic acids, proteins and polysaccharide-like substances, is one of the greatest challenges in membrane-based drinking water processes (Hashino et al., 2011; Katsoufidou et al., 2010; Sioutopoulos et al., 2010; Xiao et al., 2009). Currently, the study of NOM fouling and cleaning in water treatment was almost exclusively focused on polymeric membranes, while less research were conducted with ceramic membranes.

First of all, it is necessary to investigate the NOM fouling characteristics of ceramic membranes compared to polymeric membranes under equivalent operational conditions. The results would suggest any similarities and differences in NOM fouling mechanisms between the two membrane types. The identification of the dominant contributing resistances and relative contribution of the resistances would influence the cleaning strategy for different membrane materials. Therefore, research into the impacts of NOM fouling on ceramic membranes is needed to better clarify if and how it fouls differently than the more common polymeric membranes in drinking water treatment. To truly understand NOM fouling it is important to clarify the ease of use of fouling indices, water temperature influences, and cleaning regimes. The sections below briefly outline the needs of research in these various membrane areas. 
While NOM is generally understood to be a significant contributor to membrane fouling, the methods for analysis and prediction have also been developing over time. A great deal of effort has been made to understand the nature of the membrane fouling, develop fouling predication and quantification methods, and improve the filtration performance. Fouling prediction focuses on the utilization of fouling index methods, such as the silt density index (SDI), modified fouling index (MFI $\left.{ }_{0.45}\right)$, and modified ultrafiltration fouling index MFIUF, to assess the fouling potential of feed water and/or pretreatment efficiency prior to membrane systems. These tests are typically performed under specific standard conditions of filtration mode, membrane materials, pressure, and temperature. Fouling quantification focuses on the application of resistance in series (RIS) and unified membrane fouling index (UMFI) methods for identifying the reversible and irreversible fouling nature in an operating system. Fouling mechanisms describe how fouling occurs whether on the membrane surface, within the membrane pores, or a combination of both. Fouling characteristics focus more on identifying the operational conditions and foulant properties that play an important role in fouling during membrane filtration. Polymeric membranes have been almost exclusively dealt with in current fouling research although the same cannot be said for ceramic membranes.

Fouling indices have been developed by means of simple, short, empirical filtration tests to quantify the level or degree of membrane fouling. The $\mathrm{MFI}_{0.45}$ method is one of the most commonly used method for assessing the fouling potential of membrane feed water. This standardized method uses a $0.45 \mu \mathrm{m}$ flat sheet microfiltration (MF) membrane and typically performed at constant standard testing conditions of pressure and temperature. Water 
temperature changes seasonally and over filtration cycle of membrane systems. Thus, the actual capacity of the MFI testing and the normalization model to predict NOM fouling at non-standard testing conditions need to be clarified. Moreover, although few researchers used a flat sheet ultrafiltration (UF) membrane with the MFI testing with RO systems (Jeong and Vigneswaran, 2015; Rodriguez et al., 2015), the application of the MFI-UF method proposed by Boerlage et al. (2002), utilizing a $13 \mathrm{kDa}$ hollow fiber UF membrane, for NOM fouling prediction has not been researched in the past. Full-scale membrane systems are typically operated under conditions that are not similar to the MFI-UF testing conditions and designed with different polymeric or ceramic membrane materials. Thus, these characteristics can have significant impacts on evaluating the fouling potential of feed water, specifically if NOM is the main source of fouling.

Water temperature is another key design parameter that influence fouling and cleaning of membrane systems. Previous research on the impact of water temperature on fouling has been conducted with high pressure nanofiltration (NF) and reverse osmosis systems (RO) with temperature greater than $20^{\circ} \mathrm{C}$ and more focused on the solution diffusivity but not on changes in membrane fouling and cleaning with temperature (Zhao and Zou, 2011; Jin et al., 2009). In fact, little to no research exists that investigate water temperature effect on low pressure membranes systems. More specifically, the changes in the hydraulically and chemically irreversible and reversible fouling indices with temperature for low pressure polymeric and ceramic membrane systems need to be examined to better determine potential changes in backwash and chemical cleaning regimes for irreversible fouling control. Additionally, the actual capacity of the MFI-UF to be effectively used with the 
UMFI for NOM fouling prediction with polymeric and ceramic membranes under changes in water temperature condition needs more research. Thus, it is necessary to examine how water of different fouling indices affects polymeric and ceramic membranes in terms of fouling and cleaning efficiency. This type of research is important in order to optimize the membrane performance and ultimately reduce the treatment costs.

Chemical cleaning is an integral part of membrane systems operation. The main goal of chemical cleaning is to reduce membrane fouling, particularly irreversible fouling. The characteristics of feed water and compatibility of membrane materials influence the selection of a cleaning agent (Regula et al., 2014). Studies addressing the impacts of different cleaning parameters of sodium hypochlorite $(\mathrm{NaOCl})$ and sodium hydroxide $(\mathrm{NaOH})$ for cleaning polymeric membranes have shown that chemical concentration and cleaning sequence impact the cleaning efficiency and membrane physical structure (Puspitasari et al., 2010; Hilal et al., 2005;). High concentration of chemicals can oxidize membrane polymers and cause swelling of membrane fibers, thus, shortening the membrane lifetime (Puspitasari et al., 2010; Arkhangelsky et al., 2007). Moreover, frequent cleaning can cause changes in membrane functional groups and surface properties (Wang et al., 2018). Thus, polymeric membranes are typically restricted to lower $\mathrm{pH}$ and chlorine concentration conditions. Unlike polymeric membranes, ceramic membranes have superior chemical resistance allowing much more aggressive cleaning approaches without risk of damaging membrane integrity (Lee et al., 2014). Therefore, utilization of high $\mathrm{pH}$ cleaning in a single or stepwise approach with the $\mathrm{NaOCl}$ and $\mathrm{NaOH}$ solutions can be beneficial for 
controlling irreversible fouling of ceramic membranes for drinking water treatment applications. However, little to no research in this area.

Ozone $\left(\mathrm{O}_{3}\right)$ cleaning is another option to cleaning of ceramic membranes that is not available to polymeric membranes. The use of $\mathrm{O}_{3}$ as a pretreatment or with the backwash step with ceramic membranes for the treatment of natural waters has shown to reduce membrane fouling (Zhang et al., 2013; Kim et al., 2008; Sartor et al., 2008). However, limited to no research exists that investigate $\mathrm{O}_{3}$ CIP cleaning with respect NOM fouling control compared to conventional cleaning chemicals (i.e., $\mathrm{NaOCl}$ and $\mathrm{NaOH}$ ) with ceramic membranes. A comprehensive chemical cleaning study allows for identifying cleaning methodologies that control irreversible fouling of ceramic membranes, which can ultimately translate into a reduction in ceramic membranes operation costs due to the minimization of chemicals or treatment required.

\subsection{Research objectives and scope}

The objectives of this research are to assess the fouling response of NOM under various operating conditions and with both polymeric and ceramic membrane systems. To evaluate the capacity of the MFI-UF method in predicting NOM fouling under changes in operating conditions. Further to assess the MFI-UF effectiveness for use in complement with the UMFI with polymeric and ceramic membrane systems under changes in water temperature conditions. Moreover, this research presents side-by-side evaluation of fouling and chemical cleaning of ceramic and polymeric membranes using model NOM solutions. The specific objectives of this research were to: 
1) To assess the MFI-UF method in predicting the fouling potential of various NOM fractions commonly found in surface water sources under changes in pressure and water temperature conditions. Further to propose an empirical model that can be used to extend its useful application range for fouling studies.

2) To investigate the effects of feed water temperature on NOM fouling and cleaning of polymeric and ceramic UF membrane systems. Identify the temperature impacts on irreversible and reversible fouling ratios using the UMFI index method. Determine the capacity of the MFI-UF to be effectively used with the UMFI for predicting NOM fouling for the polymeric and ceramic UF under changing temperature conditions.

3) To quantitatively compare NOM fouling and cleaning of a tubular ceramic UF and hollow fiber polymeric UF membranes. To elucidate differences in NOM reversible and irreversible fouling mechanisms with the two membrane types.

4) To evaluate chemical cleaning regimes of a tubular ceramic UF membrane using $\mathrm{O}_{3} \mathrm{CIP}$ and conventional cleaning chemicals $(\mathrm{NaOCl}$ or $\mathrm{NaOH})$ in controlling irreversible fouling by hydrophilic and hydrophobic NOM fractions. Assess the application of $\mathrm{O}_{3} \mathrm{CIP}$ cleaning in comparison to a single, stepwise, or combined chemical cleaning approach using $\mathrm{NaOCl}$ and/or $\mathrm{NaOH}$. 


\subsection{Thesis structure}

The thesis is divided into five main stages; generally, each stage builds upon the previous. The chapters were written in journal article format; with Chapters 3, 4, 5, 6, and 7 each representing one research article.

Chapter 1: introduces the research statement and specific objectives of the research presented.

Chapter 2: is a background section that covers general information that is relevant to the research.

Chapter 3 (Published): presents the results of the first stage, which investigates the application of the MFI-UF index methods with NOM under variable testing conditions. The capacity of the MFI-UF to predict NOM fouling away from standard testing conditions was examined using regression models.

Chapter 4 (Published): presents the results of the second stage, which evaluates the impact of water temperature on fouling and cleaning behavior of a submerged polymeric UF membrane. Different model NOM solutions were used and the changes in irreversible and reversible fouling ratios with temperature were quantified using the UMFI method. Chemical cleaning of NOM was assessed for more insights into the impact of temperature on membrane resistance and permeability recoveries. The MFI-UF method was used to 
predict the fouling potential of different NOM components with temperature and its correlation with the UMFI was examined.

Chapter 5 (Submitted): presents the results of the third stage, which investigates the impact of water temperature on fouling and cleaning behavior of a tubular ceramic UF membrane. A mixture of different NOM solutions was used and the changes in irreversible to reversible fouling ratios with temperature were quantified using the UMFI method and fluorescence excitation and emission matrix (FEEM) analysis. The application of the MFIUF method for use with ceramic membranes in complement with the UMFI analysis was assessed with the NOM mixture solution.

Chapter 6 (Published): presents the results of the fourth stage, which compares fouling and cleaning behaviors of a ceramic and polymeric UF membranes with different NOM components. Membranes performance was evaluated using the RIS method, for respective differences in reversible and irreversible fouling mechanisms, NOM retention, carbon balance, and FEEM analysis of backwash and chemical wash water.

Chapter 7 (Submitted): presents the results of the final stage, which examines chemical cleaning of a tubular ceramic UF membrane using $\mathrm{O}_{3}$ in comparison to conventional cleaning chemicals $(\mathrm{NaOCl}$ and $\mathrm{NaOH})$. Hydrophobic and hydrophilic NOM solutions were used, and the cleaning efficiency was assessed using the UMFI method and carbon balance of chemical wash water. 
Chapter 8: summarizes the research work with a focus on significant conclusions and contributions and potential future work.

\subsection{Articles summary and authors contribution}

Article 1: Application of MFI-UF Fouling Index with NOM Fouling under Various Operating Conditions

Authors: Mohammad T. Alresheedi ${ }^{1}$, Onita D. Basu ${ }^{1}$

Published: Journal of Desalination and Water Treatment

Mohammad T. Alresheedi: $\quad$ First Author, Corresponding Author

Onita D. Basu: Second Author

${ }^{1}$ Department of Civil and Environmental Engineering, Carleton University, 1125 Colonel by Drive, Ottawa, ON, K1S 5B6.

\section{Authors contribution:}

Author 1: Mohammad T. Alresheedi

- Designed and performed the experiments

- Collected the data

- Performed data analysis

- Wrote the paper

Author 2: Dr. Onita D. Basu

- Contributed to experimental design and data analysis

- Contributed to the paper writing

Article 2: Investigation into the Temperature Effect on NOM Fouling and Cleaning in Submerged Polymeric Membrane Systems

Authors: Mohammad T. Alresheedi ${ }^{1}$, Onita D. Basu ${ }^{1}$

Published: Journal of Desalination and Water Treatment

Mohammad T. Alresheedi: $\quad$ First Author, Corresponding Author

Onita D. Basu: $\quad$ Second Author 
${ }^{1}$ Department of Civil and Environmental Engineering, Carleton University, 1125 Colonel by Drive, Ottawa, ON, K1S 5B6.

\section{Authors contribution:}

Author 1: Mohammad T. Alresheedi

- Designed and performed the experiments

- Collected the data

- Performed data analysis

- Wrote the paper

Author 2: Dr. Onita D. Basu

- Contributed to experimental design and data analysis

- Contributed to the paper writing

\section{Article 3: Effects of Feed Water Temperature on Irreversible Fouling of Ceramic}

\section{Ultrafiltration Membranes}

Authors: Mohammad T. Alresheedi ${ }^{1}$, Onita D. Basu ${ }^{1}$

Submitted to: Journal of Separation and Purification Technology

Mohammad T. Alresheedi: $\quad$ First Author, Corresponding Author

Onita D. Basu: $\quad$ Second Author

${ }^{1}$ Department of Civil and Environmental Engineering, Carleton University, 1125 Colonel by Drive, Ottawa, ON, K1S 5B6.

\section{Authors contribution:}

Author 1: Mohammad T. Alresheedi

- Designed and performed the experiments

- Collected the data

- Performed data analysis

- Wrote the paper

Author 2: Dr. Onita D. Basu 
- Contributed to experimental design and data analysis

- Contributed to the paper writing

\section{Article 4: Comparisons of NOM Fouling and Cleaning of Ceramic and Polymeric}

\section{Membranes during Water Treatment}

Authors: Mohammad T. Alresheedi ${ }^{1}$, Benoit Barbeau ${ }^{2}$, Onita D. Basu ${ }^{1}$

Published: Journal of Separation and Purification Technology

Mohammad T. Alresheedi: $\quad$ First Author, Corresponding Author

Benoit Barbeau Second Author

Onita D. Basu: Third Author

${ }^{1}$ Department of Civil and Environmental Engineering, Carleton University, 1125 Colonel by Drive, Ottawa, ON, K1S 5B6.

2 Department of Civil, Geological and Mining Engineering, Polytechnique, C.P. 6079

Succursale Centre-Ville, Montreal, QC, H3C 3A7.

\section{Authors contribution:}

Author 1: Mohammad T. Alresheedi

- Designed and performed the experiments

- Collected the data

- Performed data analysis

- Wrote the paper

Author 2: Dr. Benoit Barbeau

- Contributed to experimental design and data analysis

- Contributed to the paper writing

Author 3: Dr. Onita D. Basu

- Contributed to experimental design and data analysis

- Contributed to the paper writing 
Article 5: Chemical Cleaning of Ceramic Ultrafiltration Membranes - Ozone versus Conventional Cleaning Chemicals

Authors: Mohammad T. Alresheedi ${ }^{1}$, Onita D. Basu ${ }^{1}$, Benoit Barbeau ${ }^{2}$

Submitted to: Journal of Chemosphere

Mohammad T. Alresheedi: $\quad$ First Author, Corresponding Author

Onita D. Basu: $\quad$ Second Author

Benoit Barbeau Third Author

${ }^{1}$ Department of Civil and Environmental Engineering, Carleton University, 1125 Colonel by Drive, Ottawa, ON, K1S 5B6.

2 Department of Civil, Geological and Mining Engineering, Polytechnique, C.P. 6079

Succursale Centre-Ville, Montreal, QC, H3C 3A7.

\section{Authors contribution:}

Author 1: Mohammad T. Alresheedi

- Designed and performed the experiments

- Collected the data

- Performed data analysis

- Wrote the paper

Author 2: Dr. Onita D. Basu

- Contributed to experimental design and data analysis

- Contributed to the paper writing

Author 3: Dr. Benoit Barbeau

- Contributed to experimental design and data analysis

- Contributed to the paper writing 


\section{Chapter 2}

\section{Literature Review}

The main goal of all water treatment technologies is to remove pollutants that can pose health risks. Natural surface water and ground water sources are impacted by different sources of contamination such as seasonal precipitation and surrounding industrial and agricultural activities. Therefore, most water sources can contain dissolved and suspended contaminants that are harmful to human health. The Environmental Protection Agency (EPA) categorized drinking water contaminants into chemical and microbiological. Chemical contaminants include organic and inorganic compounds, heavy metals, disinfection by-products, and micro pollutants, such as pharmaceuticals and personal care products. Microbiological contaminants include coliform bacteria, E. coli, giardia, cryptosporidium, and viruses. The use of polymeric membranes in drinking water treatment has been commonly accepted as an effective technology to remove most of the chemical and microbiological contaminants. More recently, ceramic membranes have gained considerable attention in the drinking water industry due to their robustness and unique mechanical, thermal, and chemical properties. Among all water contaminants, the presence of natural organic matter $(\mathrm{NOM})$ in source water and their removal are critical issues in the operation of water treatment systems specifically membrane processes. The presence of NOM in source water is known to significantly contribute to the poor production efficiency of membranes in terms of separation performance and permeate flux. This chapter presents background information and discussion on NOM characterization, NOM fouling and cleaning, and their removals using membrane processes, with a focus on low pressure polymeric and ceramic microfiltration (MF) and ultrafiltration (UF) membranes for 
drinking water treatment applications. The literature discussion is mainly on that material which is not included in other chapters since each chapter already contains a literature review that is relevant to that specific set of objectives and experimental results. Cited references are in the list of references at the end of the thesis.

\subsection{Natural Organic Matter (NOM)}

\subsubsection{Characteristics of NOM in water}

NOM is a complex matrix of organic compounds and a key component in aquatic environments. NOM is derived both from the breakdown of terrestrial plants and as the byproduct of bacteria and algae. It consists of a range of compounds with a wide variety of chemical compositions, molecular size, molecular weight, and structure. NOM found in natural waters can be categorized into two categories: hydrophobic and hydrophilic components. The hydrophobic part, known as humic substances, represents up to $50 \%$ of total organic carbon (TOC) in most water. Hydrophobic NOM is rich in aromatic carbon, phenolic structures, and conjugated double bonds (Zularisam et al., 2006). Hydrophilic NOM contains a higher proportion of aliphatic carbon such as carbohydrates, sugars, and amino acids (Guo et al., 2012; Zularisam et al., 2006). The presence of NOM in water bodies is not known to have any direct effects on human health; however, it has significant impacts on many aspects of water treatment including performance of treatment processes, chemical usage, and the biological stability of the water. NOM can affect water properties such as color, taste, and odor. NOM can react with disinfectants used in water treatment, such as chlorine, and produce undesirable disinfection by-products (DBPs) such as trihalomethane (THM) and haloacetic acids (HAA), which are known to be carcinogenic 
to humans (Trang et al., 2012; Shao et al., 2011; Lowe and Hossain, 2008). NOM can also impact the treatment process design such as coagulation and disinfection by increasing coagulant and disinfectant dose requirements (Matilainen et al., 2011). Therefore, NOM removal is essential to meet stricter drinking water treatment regulations and overcome problems with water quality.

Membrane processes are considered reliable option for NOM removal during drinking water treatment. In fact, NOM has been identified as a major foulant during membrane filtration, which causes more fouling than any other water constituents due to its adsorptive capacity on the membrane surface (Shao et al., 2011; Zularisam et al., 2006). The presence of NOM in water is believed to be responsible for the reduction in membrane productivity during operation and shortening the lifetime of the membrane unit. NOM fractions that have been linked to fouling of polymeric MF and UF membranes as humic acids, protein, and polysaccharides substances (Wang X. et al., 2018; Hashino et al., 2011; Katsoufidou et al. 2010; Sioutopoulos et al. 2010). However, it is not clear which fraction of NOM is the dominant foulant. Some studies reported that humic acid is the fraction of dissolved organics that caused severe fouling due to adsorption (Xiao et al, 2012; Shao et al., 2011; Zularisam et al., 2011); whereas other studies found that protein is the dominant organic fraction in membrane fouling (Liu et al., 2011; Henderson et al., 2008). Recent studies have reported that sodium alginate had a significant contribution to fouling than humic acid and protein during surface water treatment (Katsoufidou et al., 2010). It is clear that humic, proteins, and polysaccharides NOM fractions are the main contributor to membrane fouling. 


\subsubsection{Measurements of NOM in water}

Characterization of NOM in terms of quantity and composition are useful for NOM optimal removal. In practice, NOM is usually represented by the measurement of total organic carbon (TOC), adsorption of UV-light $\left(\mathrm{UVA}_{254}\right)$, and/or specific ultraviolet absorbance (SUVA). TOC is composed of two carbon fractions: dissolved organic carbon (DOC) and particulate organic carbon (POC). DOC is the carbon fraction remaining in a sample after filtering the sample through a $0.45 \mu \mathrm{m}$ filter whereas POC is the carbon fraction in particulate form that is retained by a $0.45 \mu \mathrm{m}$ filter. $\mathrm{UVA}_{254}$ analysis in drinking water treatment is important to understand the composition of NOM in the source water. It is commonly used to provide insight into the aromatic compound (unsaturated double-bond) such as humic acid that can produce color and contribute to the formation of DBPs during water treatment (Zularisam et al., 2006). Normally, any wavelength from 220 to $280 \mathrm{~nm}$ has been considered to be the most appropriate for NOM measurements (Matilainen et al., 2011). The wavelengths are believed to identify different chromophores of NOM. For example, absorbance at $220 \mathrm{~nm}$ is associated with both the carboxylic and aromatic chromophores, whereas, absorbance at $254 \mathrm{~nm}$ is typical for the aromatic groups, such as humic acids. SUVA can be defined as the ratio of UV to DOC and is reported as $(\mathrm{L} / \mathrm{mg}-$ $\mathrm{cm})$. It provides insight into the hydrophobicity and biodegradability of NOM in water in which higher SUVA value indicates the presence of NOM with high aromaticity (i.e. humic substances) and lower biodegradability. Previous studies reported that SUVA of NOM from natural water is in the range of 2 to $4 \mathrm{~L} / \mathrm{mg}-\mathrm{m}$ (Zularisam et al., 2006).

Another advanced approach for the characterization of NOM is the measurements of 
fluorescence excitation and emission matrix (FEEM). FEEM is a method in which the analyte molecules are excited by irradiation at a certain wavelength and the emitted radiation is measured at wavelengths range from $200 \mathrm{~nm}$ to $500 \mathrm{~nm}$ (Matilainen et al., 2011; Zepp et al., 2004). The specific excitation and emission wavelengths are the characteristics of a particular molecular conformation, called fluorophore. These fluorophores are helpful in describing the structural compositions of NOM in water (Bieroza et al., 2010; Zhang et al., 2008; Uyguner et al., 2007; Zepp et al., 2004). FEEM has received increased attention in the drinking water treatment, particularly due to its advantages such as rapid and sensitive characterization of NOM, no sample preparation is required, and the potential for online monitoring of NOM treatability (Markechova, et al., 2013; Matilainen et al., 2011).

Various studies have demonstrated the usefulness of FEEM for drinking water treatment. For example, Gone et al. (2009) used fluorescence intensities to evaluate the coagulationflocculation process efficiency for removing dissolved organic carbon (DOC). Aluminium sulphate (alum) was used as coagulant and DOC residual and fluorescence intensities were acquired. The results illustrated a linear relationship between DOC removal and fluorescence intensities. Decrease in fluorescence peaks for each raw and treated water was correlated well with measured DOC removal $\left(\mathrm{R}^{2} \approx 0.90\right)$. The study recommended the use of fluorescence spectroscopy as an analytical technique for DOC removal efficiency in water treatment. Baghoth et al. (2011) also found that coagulation significantly reduced fluorescence intensities of humic-like as well as those of the tyrosine-like components, hence, resulting in a high DOC removal. The change of the fluorescence peaks before and 
after coagulation was also used to evaluate the performance of coagulation process at different pH levels (Bieroza et al., 2011; Bieroza et al., 2010). Moreover, FEEM analysis was used to investigate the characteristics of DOC for the formation of DBPs (Hao et al., 2012; Pifer and Fairey, 2012). Results indicated good correlation between the measured fluorescence intensities and the DBPs concentration in water $\left(\mathrm{R}^{2} \approx 0.85\right)$. In addition, fluorescence analysis can provide insights into the composition of NOM responsible for fouling during membrane filtration and that cause short and/or long-term impact on membrane performance. Peldszus et al. (2011) used FEEM analysis to identify water constituents responsible for reversible and irreversible fouling of ultrafiltration (UF) membrane and the effect of biofiltration pre-treatment on membrane fouling. Protein-like substances were found to highly correlate with irreversible fouling of the UF membrane. It was suggested that fouling transition from reversible to irreversible fouling was dependent on feed water composition and operating time. Direct biofiltration reduced the protein-like fluorescence peaks indicating the removal of protein from the membrane feed water, thus, reduced the irreversible fouling.

\subsection{Membrane Filtration Processes for Drinking Water Treatment}

The main goal of membrane filtration is to act as a physical barrier, allowing some constituents to pass through the membrane while blocking the passage of others. In membrane filtration, the water is pushed through a semi-permeable membrane. The water that passes through the membrane is called permeate or filtrate while the water remaining on the feed side is called retentate. The movement of materials across a membrane requires a driving force such as gradients in concentration or pressure across the membrane. 
Membrane processes commonly used in drinking water treatment applications use pressure as the driving force. There are four types of pressure driven membrane processes: MF, UF, NF and RO. MF and UF, known as low-pressure membranes, are commonly used to remove suspended particles, a fraction of NOM, and microbes such as viruses, E. coli, giardia, and cryptosporidium. NF and RO, known as high-pressure membranes, are commonly employed to remove NOM, DBPs and dissolved ions such as salt. The distinction between all types of membranes is typically based upon the molecular weight cut off (MWCO) or pore size. MWCO is typically defined as molecular weight of a solute that has a $90 \%$ rejection coefficient for a given membrane (Van der Bruggen et al., 2003). The characteristics of pressure driven membranes are summarized in Table 2.1.

Table 2.1- Pressure driven membranes (Adapted from Bruggen et al., 2003)

\begin{tabular}{|c|c|c|c|c|}
\hline & MF & UF & NF & RO \\
\hline $\begin{array}{c}\text { Pore size } \\
\text { (MWCO) }\end{array}$ & $\begin{array}{c}0.1-10 \mu \mathrm{m} \\
(>100,000 \mathrm{Da})\end{array}$ & $\begin{array}{c}0.01-0.1 \mu \mathrm{m} \\
(1000-100,000 \\
\text { Da) }\end{array}$ & $\begin{array}{c}1-2 \mathrm{~nm} \\
(200-400 \mathrm{Da})\end{array}$ & $\begin{array}{c}<1 \mathrm{~nm} \\
(50-200 \mathrm{Da})\end{array}$ \\
\hline $\begin{array}{c}\text { Applied } \\
\text { pressure, } \\
\text { psi (bar) }\end{array}$ & $\begin{array}{c}1.5-30 \\
(0.1-2)\end{array}$ & $\begin{array}{c}1.5-75 \\
(0.1-5)\end{array}$ & $\begin{array}{c}45-300 \\
(3-20)\end{array}$ & $\begin{array}{c}75-1800 \\
(5-120)\end{array}$ \\
\hline Rejection & $\begin{array}{c}\text { algae, } \\
\text { protozoa } \\
\text { Particles/turbidity } \\
\text { bacteria, }\end{array}$ & $\begin{array}{c}\text { Dissolved } \\
\text { organic matter, } \\
\text { Particles, } \\
\text { viruses }\end{array}$ & $\begin{array}{c}\text { Dissolved } \\
\text { organic matter, } \\
\text { ions, particles }\end{array}$ & $\begin{array}{c}\text { Dissolved } \\
\text { organic matter, } \\
\text { ions, particles }\end{array}$ \\
\hline Application & $\begin{array}{c}\text { Surface water/ } \\
\text { Groundwater }\end{array}$ & $\begin{array}{c}\text { Surface water/ } \\
\text { Groundwater }\end{array}$ & $\begin{array}{c}\text { Groundwater/ } \\
\text { Brackish water }\end{array}$ & $\begin{array}{c}\text { Seawater } \\
\text { Desalination }\end{array}$ \\
\hline
\end{tabular}

For water treatment applications, membranes are constructed from organic (polymeric) or inorganic (ceramic) materials. Polymeric membranes largely dominate the application of membrane filtration in drinking water treatment. These types of polymeric membrane 
materials can be classified into two categories: hydrophilic polymers such as cellulose acetate (CA) and polysulfone (PS), and hydrophobic membranes such as polyethersulfone (PES), polyvinylidene fluoride (PVDF), and polyacrylonitrile (PAN). Polymeric membranes have low cost and can be manufactured in different configurations such as hollow fibers, flat sheet, spiral-wound, and tubular. However, in general they have low resistance against aggressive chemical cleaning conditions (i.e. $\mathrm{pH}>11$ ) cleaning agents (i.e. chlorine) and low stability at high temperature and high pressure (Regula et al., 2014). In addition, due to their surface hydrophobicity, polymeric membranes were reported to have higher tendency to foul which limits their use without proper pretreatment or surface modification (Shao et al., 2011; Wei et al., 2006).

Ceramic membranes are composed of inorganic materials and are available as metal oxides such as aluminium oxide or alumina $\left(\alpha-\mathrm{Al}_{2} \mathrm{O}_{3}\right.$ and $\left.\gamma-\mathrm{Al}_{2} \mathrm{O}_{3}\right)$, zirconium dioxide $\left(\mathrm{ZrO}_{2}\right)$, titanium dioxide $\left(\mathrm{TiO}_{2}\right)$, and silicon carbide $(\mathrm{SiC})$. These types of membranes have an asymmetric structure, where there is a distinct transition between the dense filtration layer and the support structure, and multi-channel elements. Ceramic membranes are commonly manufactured in flat sheet or tubular configurations. These types of membranes are known for their superior mechanical, chemical, and thermal properties compared to polymeric membranes (Lee and Kim, 2014; Xia et al., 2013). Mechanical stability allows for higher fluxes, higher backwash pressures, and longer life span. The high chemical resistance allows for higher concentrations and longer exposure times to chemicals and the operation at higher $\mathrm{pH}$ conditions (i.e., $\mathrm{pH}>11$ ). Thermal stability allows for operation at high water temperatures. However, ceramic membranes currently impose higher capital costs 
compared to polymeric membranes which has thus far largely limited their use to industrial applications (e.g., food and beverage, oil and gas produced waters) (Vasanth et al., 2013; Zhou et al., 2010). However, due to their inherent operational advantages, the use of ceramic membrane for drinking water treatment is expected to grow over the next years.

\subsection{Membrane Fouling: Source, Mechanism, and Assessment}

\subsubsection{Fouling source}

Fouling is a common yet serious problem in drinking water treatment using membrane processes. Membrane fouling can be defined as the accumulation of unwanted feed impurities (e.g. particulates, colloidal matters, organic and inorganic materials, and microorganisms) on the membrane surface or within membrane pores. The adverse effects of fouling include decrease in the efficiency of separation performance (i.e., low rejection), decrease in permeate flux, and increase in transmembrane pressure (TMP). These effects can eventually impact the economics of membrane processes by increasing the frequency of cleaning and energy requirements. Previous studies have demonstrated that membrane fouling is influenced by the membrane properties (i.e. hydrophobicity, surface charge and MWCO), source water composition, and mode of operation (Kenari and Barbeau, 2016; Guo et al., 2012; Sentana et al., 2011; Shao et al., 2011). Among these factors, water composition plays an important role in fouling. Previous studies on fouling have classified membrane foulants into four categories as shown in Table 2.2. These foulants can cause either reversible or irreversible fouling. The loss in membrane performance due to reversible fouling can be restored through appropriate physical cleaning methods (i.e. backwashing and air scour) and chemical cleaning (using chemical agents). On the other 
hand, irreversible fouling cannot be removed through physical and chemical cleaning methods and the membrane must go through extensive chemical cleaning or to be replaced.

Table 2.2- Classification of membrane foulants (Adapted from Guo et al., 2012)

\begin{tabular}{|c|c|c|}
\hline Source of Foulant & Example & Fouling Mechanism \\
\hline Organics & $\begin{array}{c}\text { organic compounds } \\
\text { (e.g. humic and proteins) }\end{array}$ & Adsorption, pore blocking \\
\hline Inorganics & $\begin{array}{c}\text { Inorganic compounds } \\
\text { (e.g. calcium and silica) }\end{array}$ & $\begin{array}{c}\text { Precipitate onto the membrane } \\
\text { surface (scaling) }\end{array}$ \\
\hline Colloids & $\begin{array}{c}\text { organic or inorganic } \\
\text { colloids }\end{array}$ & Cake formation, pore blocking \\
\hline Biological Organisms & Algae, bacteria & Biofouling (biofilm formation) \\
\hline
\end{tabular}

\subsubsection{Fouling mechanism}

Over the past decades, researchers have identified several filtration models known as blocking law models that describe the relation between flux, TMP, and fouling during membrane filtration. Blocking models describe four main mechanisms of fouling: cake formation, intermediate blocking, standard blocking, and complete blocking (Blankert et al., 2006; Hermia, 1982). Fouling due to cake formation is based on the assumption that particles that are larger than the membrane pore size are retained due to cake formation on the membrane surface. Other particles will tend to deposit on the cake layer which will eventually increase the filtration resistance. In the intermediate blocking model, large and small particles tend to deposit on previously deposited particles or can narrow membrane pores. Standard blocking occurs when particles smaller than membrane pore size deposit on the internal membrane pores. Complete blocking is based on the assumption that particles tend to block the membrane pores by sealing the pores completely (Guo et al., 2012). Figure 2.1 illustrates different membrane fouling mechanisms. 


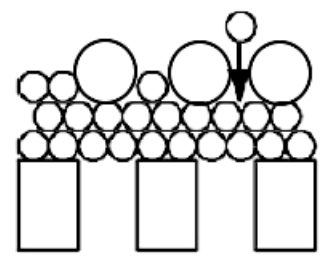

(A)

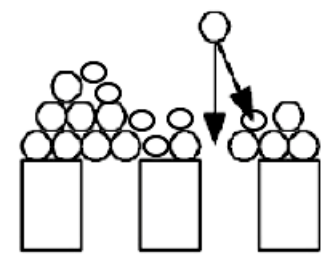

(B)

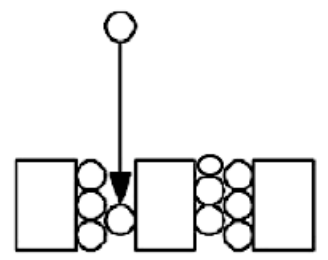

(C)

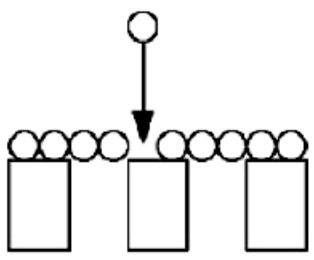

(D)

Figure 2.1 - Illustration of membrane fouling mechanisms (blocking models): (A) Cake formation, (B) Intermediate blocking, (C) Standard blocking, (D) Complete blocking. Adapted from Blankert et al. (2006).

\subsubsection{Fouling assessment}

\subsubsection{Resistance in series (RIS)}

During membrane filtration, foulants in source water can cause either reversible and/or irreversible fouling resistance. The RIS method, Equation 2.1, (Crittenden et al., 2005), is commonly applied to estimate each contributing fouling resistance using operational data such as flux, pressure, and temperature.

$\mathrm{J}=\frac{\Delta \mathrm{P}}{\mu \mathrm{R}_{\mathrm{t}}}=\frac{\Delta \mathrm{P}}{\mu\left(\mathrm{R}_{\mathrm{m}}+\mathrm{R}_{\mathrm{hr}}+\mathrm{R}_{\mathrm{cr}}+\mathrm{R}_{\mathrm{cir}}\right)}$

Equation (2.1)

Where $\mathrm{J}$ is the filtration flux $(\mathrm{m} / \mathrm{s}) ; \Delta \mathrm{p}$ is the applied transmembrane pressure (psi); $\mu$ is the water viscosity ( $\mathrm{kg} / \mathrm{m} . \mathrm{s})$; and $\mathrm{R}_{\mathrm{t}}$ is total resistance $\left(\mathrm{m}^{-1}\right)$. $\mathrm{R}_{\mathrm{t}}$ is a function of the intrinsic membrane resistance $\left(\mathrm{R}_{\mathrm{m}}\right)$; hydraulically reversible resistance $\left(\mathrm{R}_{\mathrm{hr}}\right)$ that can be removed by backwash, and hydraulically irreversible resistance $\left(\mathrm{R}_{\text {hir }}\right)$ that resist backwash. $\mathrm{R}_{\text {hir }}$ can be divided into chemically reversible resistance $\left(\mathrm{R}_{\mathrm{cr}}\right)$ which can be removed by chemical 
cleaning, and chemically irreversible resistance $\left(\mathrm{R}_{\mathrm{cir}}\right)$ which cannot be removed by chemical cleaning. The later resistant is undesirable as it causes long term loss in membrane permeability and shorten the lifetime expectancy of the membrane unit. $R_{h r}$ and $R_{h i r}$ are calculated by collecting flux and pressure data before and immediately after backwash respectively; whereas $\mathrm{R}_{\mathrm{cr}}$ and $\mathrm{R}_{\mathrm{cir}}$ are calculated by collecting flux and pressure data before and after chemical cleaning, respectively.

\subsubsection{Unified membrane fouling index (UMFI)}

The UMFI model, Equation 2.2 (Huang et al., 2008) has been introduced as a method for calculating reversible and irreversible fouling based on the relation between the normalized specific flux and specific permeate volume.

$\frac{1}{\mathrm{~J}_{\mathrm{s}}{ }^{\prime}}=1+(\mathrm{UMFI}) \mathrm{x} \mathrm{V}_{\mathrm{S}}$

Where:

$\mathrm{J}_{\mathrm{S}}$ : is the normalized specific flux, $\mathrm{Js}^{\prime}=(\mathrm{J} / \mathrm{P}) /\left(\mathrm{J}_{0} / \mathrm{P}_{0}\right)$, (unitless)

$V_{\mathrm{S}}$ : is the specific permeate volume $\left(\mathrm{m}^{3} / \mathrm{m}^{2}\right)$

UMFI: is an estimate of the extent of fouling $\left(\mathrm{m}^{-1}\right)$.

During membrane filtration, the UMFI is divided into different fouling indices as shown in Equation 2.3.

$\mathrm{UMFI}_{\mathrm{f}}=\mathrm{UMFI}_{\mathrm{hr}}+\mathrm{UMFI}_{\mathrm{hir}}=\mathrm{UMFI}_{\mathrm{hr}}+\mathrm{UMFI}_{\mathrm{cr}}+\mathrm{UMFI}_{\mathrm{cir}}$ Equation (2.3) Where:

UMFIf: is the total fouling resistance index $\left(\mathrm{m}^{-1}\right)$ 
$\mathrm{UMFI}_{\mathrm{hr}}$ : is hydraulically reversible fouling resistance index (removable by backwash, $\mathrm{m}^{-1}$ ) $\mathrm{UMFI}_{\text {hir: }}$ is hydraulically irreversible fouling resistance index (remained after backwash, $\left.\mathrm{m}^{-1}\right)$. UMFI $\mathrm{Uir}_{\text {ir }}$ can be divided into $\mathrm{UMFI}_{\mathrm{cr}}$ and $\mathrm{UMFI}_{\mathrm{cir}}$.

$\mathrm{UMFI}_{\mathrm{cr}}$ is chemically reversible fouling resistance index (removable by chemical cleaning, $\left.\mathrm{m}^{-1}\right)$

UMFI $_{\text {cir: }}$ is chemically irreversible fouling resistance index (remained after chemical cleaning, $\left.\mathrm{m}^{-1}\right)$.

Each fouling index is calculated as following: $\mathrm{UMFI}_{\mathrm{f}}$ represents the slope of the $1 / \mathrm{J}_{\mathrm{S}}$ ' versus $V_{S}$ data from the start to end of filtration. $\mathrm{UMFI}_{h i r}$ represents the slope of the $1 / \mathrm{J}_{\mathrm{S}}$ ' versus Vs data after the backwash step. $\mathrm{UMFI}_{\mathrm{hr}}$ is the difference between $\mathrm{UMFI}_{\mathrm{f}}$ and $\mathrm{UMFI}_{h i r}$. $\mathrm{UMFI}_{\text {cir }}$ represents the slope of the $1 / \mathrm{J}_{\mathrm{S}}$ versus $\mathrm{V}_{\mathrm{S}}$ data the chemical cleaning step. $\mathrm{UMFI}_{\mathrm{cr}}$ is the difference between $\mathrm{UMFI}_{\text {hir }}$ and $\mathrm{UMFI}_{\text {cir }}$.

\subsubsection{Fouling indices}

Membrane fouling is an inevitable problem impairing all membrane processes. Over the past several years, researchers have developed fouling prediction methods that can be employed to assess the fouling tendency of membrane feed water. The silt density index (SDI), modified fouling index (MFI ${ }_{0.45}$ ), and modified ultrafiltration fouling index (MFIUF) methods have been proposed subsequently (ASTM, 2014; Boerlage et al., 2002; Schippers and Verdouw, 1980). These short filtration tests are used to estimate a fouling index value that give a general indication about the treatability of feed water or the need for pretreatment prior to the membrane unit. 


\subsection{Silt density index (SDI)}

The SDI measurement is performed according to a standard method (ASTM-D4189 07/2014). The feed water is pumped into a flat sheet MF membrane with a pore size of $0.45 \mu \mathrm{m}$ in dead-end filtration mode at a constant pressure of 2 bar (30 psi) and constant water temperature of $20^{\circ} \mathrm{C}$. The SDI is a time based test in which the plugging rate of a membrane filter $(\mathrm{P})$ is estimated by measuring the time required to collect the first $500 \mathrm{~mL}$ of filtrate sample at the beginning of filtration $\left(\mathrm{t}_{\mathrm{i}}\right)$ and the time needed for collecting a second $500 \mathrm{~mL}$ of filtrate sample $\left(\mathrm{t}_{\mathrm{f}}\right)$, usually after 5,10 , or 15 minutes of filtration $(\mathrm{T})$. The SDI value can be calculated using Equation 2.4 (ASTM, 2014). A schematic representation of the SDI setup is shown in Figure 2.2.

$\mathrm{SDI}=\frac{\% \mathrm{P}}{\mathrm{T}}=\frac{\left(1-\frac{\mathrm{ti}}{\mathrm{tf}}\right) \times 100}{\mathrm{~T}}$

Equation (2.4)

The SDI value represents the expected percentage of flux decline per minute of filtration for a specific membrane system. In general, feed water with the SDI value $<3 \% / \mathrm{min}$ would result in lower fouling rates and consider suitable for membrane feed without pretreatment. For feed water having an SDI value $>3 \% / \mathrm{min}$, it is preferable to be pretreated prior to the membrane process in order to control fouling (Koo et al., 2012). The common pretreatment methods include flocculation, PAC adsorption, ozonation, and/or media filtration. 


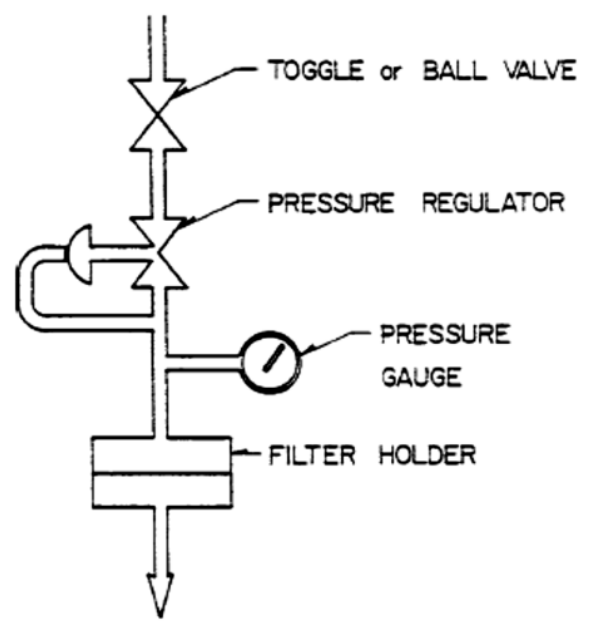

Figure 2.2 - SDI setup (adapted from ASTM-D4189 - 07/2014)

Previous research and field observations have reported major drawbacks of the SDI measurement. Lack of a correlation between the concentration of particles in water and the SDI value estimated. some membrane plants experienced severe fouling when receiving feed water of a low SDI value (i.e. low fouling potential) whereas water with low turbidity had high SDI values (Rachman et al., 2011; Burashid and Hussain, 2004; Ando et al., 2003). Severe colloidal fouling problems were observed in a RO membrane treating feed water with an SDI $<1 \% /$ min (Yiantsios et al., 2005). RO permeate with turbidity around 0.06 NTU have an SDI around 2\%/min (Rachman et al., 2011). In addition, the SDI test is based on a comparison between two flow rates at two different times, which are at the beginning and at the end of filtration. Thus, the distinction between different fouling mechanisms, (i.e. pore blocking and cake filtration) is not considered during testing. As a consequence of the lack of a filtration model supporting the calculation of the SDI, the predictability of the fouling rate by the SDI is questionable (Koo et al., 2012). The lack of agreement between SDI operational conditions and membranes may also result in an overestimation or underestimation of the fouling potential of feedwater. The SDI testing 
was also found to be influenced by the membrane resistance and pore size (Habib et al., 2013; Alhadidi et al., 2011; Rachman et al., 2011). In addition, while the SDI test is mainly used as an indicator of particulates and colloids fouling, other water quality attributes such as NOM also contribute to fouling, which leads to some of disconnect between predicted SDI values and actual observed fouling in the field. Theoretically, water filtered with MF and UF membranes would achieve better quality of permeate whereby the SDI usually should be $<3 \% / \mathrm{min}$. However, Mosset et al. (2008) study found that NOM fouling had occurred in the membrane system despite the fact that a low value of SDI (i.e., $\mathrm{SDI}_{15}$ of $2 \% / \mathrm{min}$ ) was obtained upon pretreatment with UF membrane. From these studies, the reliability of the SDI to predict NOM fouling under changes in testing conditions is questionable.

\subsection{Modified fouling index $\left(\mathrm{MFI}_{0.45}\right)$}

The $\mathrm{MFI}_{0.45}$ is determined using the same equipment setup for the SDI (refer to Figure 2.2), however is performed differently which results in different data on fouling. The volume of filtered water is recorded at an interval of 30 seconds over a 15 minutes filtration period (ASTM, 2016; Schippers and Verdouw 1980). Equation 2.5 can be used to calculate the $\mathrm{MFI}_{0.45}$. Where $\mathrm{t}$ is the filtration time (s); $\mathrm{V}$ is permeate produced $\left(\mathrm{m}^{3}\right) ; \Delta \mathrm{P}$ is the pressure (bar); $\mu$ is the viscosity of water $(\mathrm{kg} / \mathrm{m} \mathrm{s})$; $A$ is the membrane area $\left(\mathrm{m}^{2}\right) ; \mathrm{R}_{\mathrm{m}}$ is the membrane resistance $\left(\mathrm{m}^{-1}\right)$; $\alpha$ is the specific cake resistance $(\mathrm{m} / \mathrm{kg})$; and $\mathrm{C}_{\mathrm{b}}$ is the bulk concentration $\left(\mathrm{kg} / \mathrm{m}^{3}\right)$. The $\mathrm{MFI}_{0.45}$ is defined as the slope of the inverse flow rate (t/V) versus permeate volume (V). 
$\frac{\mathrm{t}}{\mathrm{V}}=\frac{\mu \mathrm{R}_{\mathrm{m}}}{\Delta \mathrm{PA}}+\left(\frac{\mu \mathrm{C}_{\mathrm{b}} \alpha}{2 \Delta \mathrm{PA} \mathrm{A}^{2}}\right) \mathrm{V}=\frac{\mu \mathrm{R}_{\mathrm{m}}}{\Delta \mathrm{PA}}+(\mathrm{MFI}) \mathrm{V} \quad$ Equation (2.5)

Unlike the SDI, the $\mathrm{MFI}_{0.45}$ is based on observations that different fouling mechanisms occur during testing (Boerlage et al 2002; Schippers and Verdouw, 1980). The MFI 0.45 graph (Figure 2.3) is divided into three regions. The first region represents membrane pore blocking by particles. The second region represents cake formation while the third region represents cake formation with compression. The $\mathrm{MFI}_{0.45}$ is the slope of the line in the second region considering that cake formation is the dominant fouling mechanism during filtration. Feed water having an $\mathrm{MFI}_{0.45}$ between $0-10 \mathrm{~s} / \mathrm{L}^{2}$ is acceptable for membrane feed (Koo et al., 2012). However, the $\mathrm{MFI}_{0.45}$ value was found to be dependent on the membrane filter pore size. Schippers and Verdouw, (1980) found that the $\mathrm{MFI}_{0.45}$ value for river water increased with decreasing membrane pore size from $0.8-0.05 \mu \mathrm{m}$. The increase of $\mathrm{MFI}_{0.45}$ was attributed to the presence of smaller colloids in the river water. Therefore, the $\mathrm{MFI}_{0.45}$ was found to be unable to account for small colloids. Lower $\mathrm{MFI}_{0.45}$ values were obtained for the membrane with $0.45 \mu \mathrm{m}$ pore size or higher due to its failure to capture the smaller particles (Schippers and Verdouw, 1980). In addition, the measurement method of $\mathrm{MFI}_{0.45}$ is relatively more complicated than the SDI test. MFI involves a more complex test analysis, whereby the value of $\mathrm{MFI}_{0.45}$ could only be obtained by plotting a graph and estimating the slope of the linear region which represents the $\mathrm{MFI}_{0.45}$. That is why SDI testing is usually conducted by operators on site (Koo et al., 2013). 


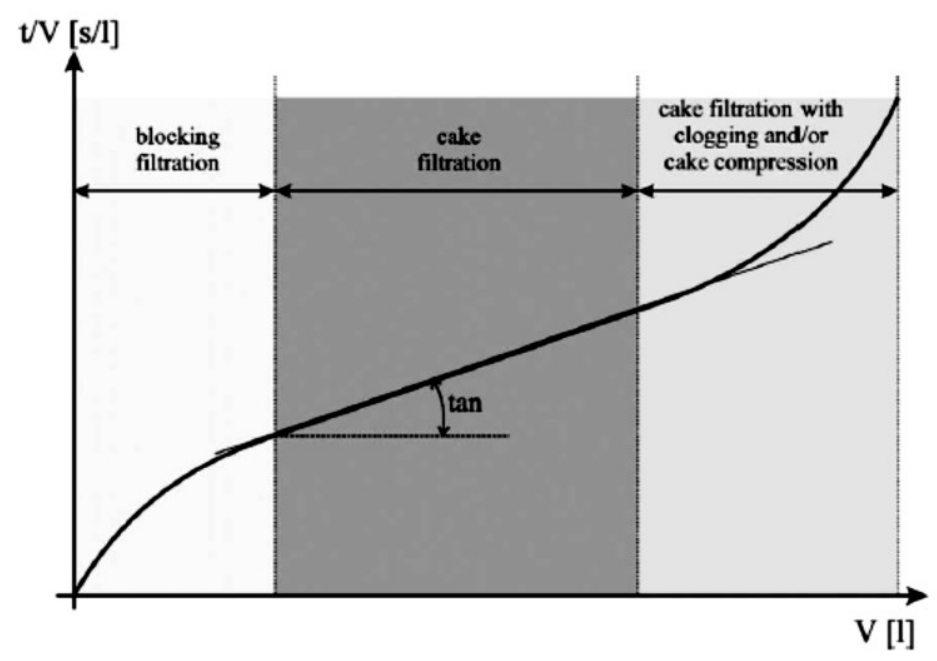

Figure 2.3 - Ratio of filtration time and filtrate volume $(\mathrm{t} / \mathrm{V})$ as a function of filtrate volume (V), (adapted from Boerlage et al., 2003)

\subsection{Modified ultrafiltration fouling index (MFI-UF)}

As previously noted, the SDI and $\mathrm{MFI}_{0.45}$ are the most widely applied methods to measure the fouling potential of feedwater. The two methods use specific membranes with pore size of $0.45 \mu \mathrm{m}$ but are performed differently. However, researchers have observed that smaller particles (i.e. $<0.45 \mu \mathrm{m}$ ) contribute to membrane fouling (Boerlage et al., 2002; Schippers and Verdouw,1980). It was hypothesized that these particles limit the ability of SDI and $\mathrm{MFI}_{0.45}$ in predicting fouling rate during membrane operation. Boerlage et al. (2002) developed a MFI utilizing a UF membrane (MFI-UF) to incorporate the fouling of smaller particles (i.e. $<0.45 \mu \mathrm{m}$ ). The MFI-UF is determined by pumping feed water into a PAN hollow fiber UF membrane with molecular weight cutoff (MWCO) of $13 \mathrm{kDa}$ in dead-end filtration mode at constant pressure of 2 bar and constant water temperature of $20{ }^{\circ} \mathrm{C}$ (Boerlage et al., 2003; Boerlage et al., 2002). A typical MFI-UF setup is shown in Figure 2.4 . 


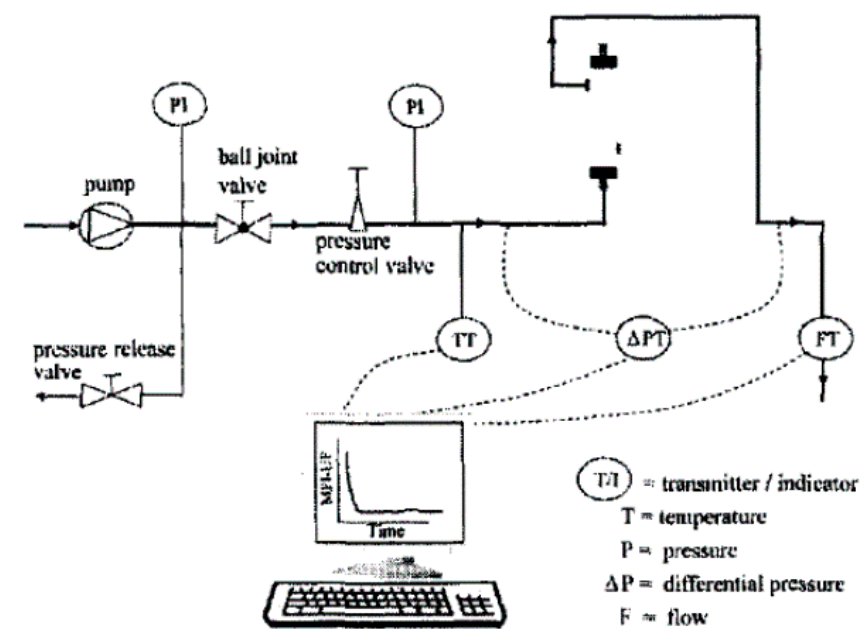

Figure 2.4 - MFI-UF equipment (adapted from Boerlage et al., 2002)

Similar to the $\mathrm{MFI}_{0.45}$, the MFI-UF value is determined graphically by plotting the inverse of the flow rate $(\mathrm{t} / \mathrm{V})$ versus cumulative filtered water volume $(\mathrm{V})$, (refer to Figure 2.3). The slope of the line in the second region (cake filtration region) is the MFI-UF. Feed water having an MFI-UF $<3000 \mathrm{~s} / \mathrm{L}^{2}$ is equivalent to $\mathrm{SDI}<3 \% / \mathrm{min}$, which is acceptable for membrane feed (Boerlage et al., 2003; Boerlage et al., 2002). The effectiveness of the MFIUF in predicting the fouling potential of RO processes was assessed using different hollow fiber UF membranes with different pore sizes (1 to $100 \mathrm{kDa})$. The values of the MFI-UF reported were 400-1400 times greater than the corresponding SDI and $\mathrm{MFI}_{0.45}$ due to the retention of smaller particles by the UF membrane (Boerlage et al., 2002). A PAN $13 \mathrm{kDa}$ UF membrane was proposed as the most promising reference membrane for the MFI-UF testing (Boerlage et al., 2002; Boerlage et al., 2003).

The utilization of the MFI-UF for assessing the fouling propensity has been carried out in recent studies with high pressure RO systems (Jeong and Vigneswaran, 2015; Ju et al., 
2015; Rodriguez et al., 2015; Villacorte et al., 2015; Taheri et al., 2013; Sim et al., 2011). For example, the MFI-UF was used to assess particulate and biofouling potential for RO membranes (Jeong and Vigneswaran, 2015; Rodriguez et al., 2015). Moreover, MFI-UF was applied to assess fouling potential of algae (Villacorte et al., 2015). Other studies (Ju et al., 2015; Taheri et al., 2013), investigated the influence of inorganic silica and calcium colloids on the MFI-UF fouling index for seawater RO systems. It should be noted that in the above studies, a flat sheet UF membrane was used which was different than the hollow fiber (13 kDa) PAN membrane proposed by Boerlage et al. (2003). Thus, the filtration configuration and rejection mechanism differ. In fact, to date, little to no research exists on the ability of the MFI-UF to predict NOM fouling for low pressure polymeric and ceramic membrane systems under changes of membrane process conditions, which is necessary for expanding the MFI-UF application for NOM fouling prediction.

\subsection{Water Contaminants Removal Using Polymeric and Ceramic Membranes}

2.4.1 Polymeric membranes applications in drinking water treatment

Polymeric MF and UF membrane processes have gained considerable acceptance in the drinking water industry over the past decades, and have demonstrated excellent capabilities for removal of particles, turbidity, microorganisms such as giardia and cryptosporidium and a certain amount of NOM. In fact, it is well known that various NOM fractions such as humic acids, proteins, and polysaccharides substances are the main contributors to polymeric membranes fouling during water treatment (Tian et al., 2013; Hashino et al., 2011; Katsoufidou et al., 2010; Xiao et al., 2009; Katsoufidou et al., 2007; Yuan and Zydney, 2000). Yuan and Zydney (2000) studied humic acid fouling during MF and found 
that humic acid was responsible for the initial stage of fouling. Furthermore, the fouling mechanism was substantially due to deposition and internal pore adsorption. Van de Ven et al., (2008) study found that the irreversible fouling resistance of the alginate increased during filtration and showed an adverse impact on membrane permeability. This can be supported by Sioutopoulos et al. (2010) who investigated alginate and humic acid fouling of a PAN membrane. Alginate resulted in a greater drop in membrane permeability than humic acid. The cake resistance of alginate and humic acid was found to increase with increasing alginate concentration. This was similar to findings by Katsoufidou et al. (2010) who found that the cake resistance of the alginate and humic acid mixture was much closer to the cake resistance of alginate alone. Xiao et al. (2009) study reported that the adsorption of protein to a PVDF membrane was much lower than the adsorption of humic acid. This was not the case in the Hashino et al. (2011) study that reported higher protein fouling with hydrophobic membranes. Tian et al. (2013) study showed that humic acid alone resulted in higher fouling than when humic acid was filtered together with a protein. From these studies, it is clear that different NOM compositions contribute differently to fouling of different polymeric membranes.

Other applications of MF and UF membranes in water treatment include microbial removal by physical sieving which is considered as the major mechanism of removal of giardia and cryptosporidium and other microbes. The pore sizes for MF and UF used in water treatment processes is lower in magnitude than the size of protozoan cysts $(4-15 \mu \mathrm{m})$, therefore, it has been generally accepted that MF and UF can provide complete removal of all protozoan cysts. Studies have demonstrated that MF and UF membranes provide log removals of 
giardia and cryptosporidium ranging from 99 to $99.9999 \%$ (2-6 log removal) (Fiksdal and Leiknes, 2006; Betancourt and Rose, 2004). However, biofouling remains as one of the biggest issue using polymeric MF and UF membranes in water treatment applications (Henderson et al., 2008).

\subsubsection{Ceramic membranes applications in drinking water treatment}

Ceramic membranes, which are made of inorganic materials, are a rapidly emerging technology due to many advantages over polymeric membranes. Ceramic membranes are superior in their mechanical and chemical characteristics, which allows for higher fluxes and backwash pressures as well as aggressive cleaning approaches without risk of damaging membrane integrity (Lee and Kim, 2014; Pendergast and Hoek, 2011). These advantages would potentially increase the operational life expectancy of ceramic membranes and decrease the cleaning and replacement requirements. The downside of ceramic membranes is the high capital costs compared to polymeric membranes which have limited the use of polymeric membranes to industrial applications that deal with challenging and high polluted waters (Vasanth et al., 2013; Zhou et al., 2010). However, due to their inherent operational advantages, the implementation of ceramic membranes for drinking water production is increasing. For example, in the United States (US), 2.5 MGD and 10 MGD plants are being designed in California and Colorado respectively (Freeman and Shorney-Darby, 2011). In Japan, over 50 ceramic membrane systems have been constructed (Freeman and Shorney-Darby, 2011; McAliley and D'Adamo, 2009). In the Middle East, ceramic membranes have been implemented as pretreatment step for NF/RO systems in desalination plants (Dramas and Groue, 2013; Hamad et al., 2013). 
Several studies have applied ceramic membranes for water treatment. For example, Konieczny et al. (2006) used coagulation and ceramic MF processes to treat water containing 7 and $10 \mathrm{mg} \mathrm{C} / \mathrm{L}$ of humic acids. Two 19- channel ceramic membranes with pore sizes of 0.1 and $0.2 \mu \mathrm{m}$ respectively, composed of a monolithic support layer of $\mathrm{TiO}_{2} / \mathrm{Al}_{2} \mathrm{O}_{3}$ and an active layer of $\mathrm{ZrO}_{2} / \mathrm{TiO}_{2}$ were used. The membrane filtration process was operated using a cross flow configuration at constant pressure. The removal of TOC by coagulation alone, ceramic MF alone and a combination of coagulation and MF processes were investigated. Ceramic MF membrane showed a complete removal of TOC (i.e. $100 \%$ ) compared to coagulation alone. However, a significant flux decline due to organic adsorption and pore blocking was observed when using MF process alone. The use of coagulation prior to the MF process showed significant improvements in fouling reduction and flux. It was suggested that performance by MF alone is not suitable for optimal operation, and when used with pre-coagulation, it can be improved with stable fluxes. This can be supported by studies (Meyn et al., 2012; Meyn et al., 2008; Lehman et al., 2007) that showed a combination of coagulation and ceramic MF processes was effective in improving DOC removal and maintaining a stable membrane operation.

A number of studies (Fan et al., 2014; Guo et al. 2013; Lehman and Liu, 2009; Kim et al., 2008) have used ozonation process prior to ceramic membranes and this is in part due to the fact that ceramic membranes have superior resistance to strong oxidants. Kim et al. (2008) investigated the effects of three ozone dosages (1.5, 5.5 and $\left.9.5 \mathrm{~g} / \mathrm{m}^{3}\right)$, cross-flow velocities $(0.11,0.47$, and $0.88 \mathrm{~m} / \mathrm{s})$ and TMP $(0.68,1.36$, and 2.03 bar $)$ on the performance of tubular ceramic UF membrane composed of $\alpha-\mathrm{Al}_{2} \mathrm{O}_{3}$ and $\mathrm{TiO}_{2}$. The ceramic membrane 
system was used to treat lake water with average TOC concentration of $11.8 \mathrm{mg} / \mathrm{L}$ under a cross flow mode. A significant improvement in permeate fluxes was observed at higher ozone dosage and cross-flow velocity and lower TMP. However, the percent removal trend of TOC across the membrane was observed to decrease with increasing ozone dosage. It was hypothesized that this was due to partial degradation of NOM to form smaller molecules that can easily pass through the membrane. Guo et al. (2013) used a flat sheet ceramic UF membrane with a pore size of 50-60 nm with ozonation and biological activated carbon filtration $(\mathrm{BAC})$ to treat highly polluted river water $(\mathrm{TOC} \approx 12-15 \mathrm{mg} / \mathrm{L})$. Ozone dose was between $2.0-2.5 \mathrm{mg} / \mathrm{L}$. Results showed that ceramic UF membrane was able to remove most of turbidity and suspended particulate matters (average effluent turbidity $\approx 0.14 \mathrm{NTU})$. Ozonation altered the molecular structure of organics which reduced membrane fouling and extended the membrane operation. In addition, the ozonation improved the biodegradability of organic matter, and helped the BAC process to remove up to $90 \%$ of the DOC available in the water.

Few papers have investigated the performance of ceramic membranes to treat water without pretreatment. Rubia et al. (2006) used a tubular UF ceramic membrane with a MWCO of $15 \mathrm{kDa}$ to treat a $10 \mathrm{mg} / \mathrm{L}$ of synthetic humic acid solution under various $\mathrm{pH}$ and ionic strengths. $99 \%$ removal of DOC was obtained at $\mathrm{pH}$ of 7.9 and low ionic strength whereas at low $\mathrm{pH}$ of 2.4 and high ionic strength, the DOC removals decreased to $74 \%$. It was concluded that the $\mathrm{pH}$ and the ionic strength conditions affected the filtration of humic acid by changing the properties of both the ceramic membrane surface and humic acid. Xia et al. (2013) used a $0.01 \mu \mathrm{m}$ tubular UF ceramic membrane to treat two sources of water: 
synthetic humic acid solutions (TOC of $2.78-9.91 \mathrm{mg} / \mathrm{L}$ ) and raw lake water (TOC of 3.9 $\mathrm{mg} / \mathrm{L})$. Membrane flux decreased with increasing humic acid concentration. It was hypothesized that increasing humic acid concentration would accelerate pore blockage and particle accumulation on the membrane surface, hence, higher fouling. The removal of humic acid from the lake water was below $20 \%$ compared to the results with the synthetic humic acid solution (> 50\%). This was related to differences in feed composition and molecular sizes (Xia et al., 2013).

Few published studies have compared the performance of ceramic membranes to polymeric membranes for drinking water treatment applications (Kenari et al., 2016; Lee et al. 2014; Hofs et al., 2011). It should be noted that these studies used ceramic and polymeric membranes with different characteristics (i.e., different pore size and surface area), different backwash procedures, and short filtration experiments ( $<5$ hours). However, the results of these studies are promising. Hofs et al., (2011) compared fouling of four ceramic $\left(\mathrm{TiO}_{2}, \mathrm{ZrO}_{2}, \mathrm{Al}_{2} \mathrm{O}_{3}\right.$, and $\left.\mathrm{SiC}\right)$ to a PES polymeric MF membrane. NOM and UV removals were around $30 \%$ for the ceramic membranes and between $13-25 \%$ for the polymeric membrane. The $\mathrm{TiO}_{2}$ and $\mathrm{SiC}$ membranes showed the lowest TMP increase compared to the $\mathrm{Al}_{2} \mathrm{O}_{3}, \mathrm{ZrO}_{2}$ and polymeric membrane. Kenari and Barbeau (2016) used ceramic and polymeric UF membranes for iron and manganese removal under changes in water chemistry. Water $\mathrm{pH}$, hardness, and ionic strength had little impact on the overall iron and manganese removal efficiencies but did contribute to fouling. Physical cleaning of iron and manganese deposits was more effective for the ceramic UF membranes compared to the polymeric one. The results of the above studies have shown a great potential for the 
utilization of ceramic membranes for drinking water production. However, little available research that focus on NOM fouling of ceramic membranes which is needed to better clarify if and how it fouls differently than the more common polymeric membranes in drinking water treatment.

\subsection{Effect of Water Temperature on Membrane Filtration Performance}

Water temperature is a key design parameter that influence membrane performance. The majority of previous studies that have examined temperature associated impacts with membranes were done with high pressure membrane systems NF and RO at temperatures greater than $20^{\circ} \mathrm{C}$ or wastewater membrane bio reactor (MBR) systems (Ma et al., 2013; Van den Brink et al., 2011; Zhao and Zou, 2011; Jin et al., 2009; Mo et al., 2008). These type of studies tends to focus more on solution viscosity and solute diffusivity challenges with operation but not changes in fouling behavior. For example, Jin et al., (2009) studied the effect of temperature on humic acid fouling with RO membranes. At higher temperatures of $35^{\circ} \mathrm{C}$, humic acid rejection was lower due to changes in the membrane structure and in the fraction of dissolved organic. Flux decline was higher at $15^{\circ} \mathrm{C}$, but was identical at 25 and $35^{\circ} \mathrm{C}$. Their study suggested that higher feed water temperature reduced energy consumption by RO system. Zhao and Zou, (2011) studied the effect of temperature on RO systems and showed that higher temperature would afford higher initial fluxes and higher water recoveries, but also caused more adverse effects on membrane scaling and cleaning. The results of Zhao and Zou (2011) are different than those reported by Jin et al. (2009), however, both studies showed the impact of temperature on RO membranes. Other studies (Ma et al., 2013; Van den Brink et al., 2011) studied the effect of temperature 
variation on membrane fouling and microbial community structure in MBRs. Higher membrane fouling rates with low fouling reversibility of proteins and polysaccharides were obtained at lower temperature of $7^{\circ} \mathrm{C}$ compared to $25^{\circ} \mathrm{C}$ (Van den Brink et al., 2011). Extracellular polymer substances (EPS) and soluble microbial products (SMPs) increased with decreasing temperature, which increased membrane fouling (Ma et al., 2013).

Studies that deal with temperature effect on low pressure polymeric and ceramic membranes are very limited (Steinhauer et al., 2015). Drinking water systems have in general neglected the interplay of water temperature and fouling on low pressure membrane systems. Although one study with low pressure membrane systems found that current standards for membrane integrity testing overestimated safety design values by not taking into account temperatures below $5{ }^{\circ} \mathrm{C}$ (Farahbakhsh and Smith, 2006), highlighting the need for more research in this area. More specifically, the changes in reversible and irreversible fouling ratios with temperature for low pressure polymeric and ceramic membranes need to be identified to help control fouling and maintain membrane productivity.

\subsection{Chemical Cleaning of Polymeric and Ceramic Membranes}

Chemical cleaning is an integral part of the operation of membrane processes. Chemical cleaning is performed to remove foulants that are not removed by physical cleaning (i.e., backwashing). This type of fouling is referred to it as chemically reversible fouling. Membrane chemical cleaning can be performed directly by immersing the fouled membrane in the chemicals (clean-in-place (CIP)) or by cleaning in conjunction with the 
backwash step (a chemical enhanced backwash (CEB)) (Shi et al., 2014). The common chemicals that are used for cleaning membranes are classified into five categories as shown in Table 2.3. The selection of a cleaning agent depends on the fouling source, compatibility of membrane materials and recommendations of membrane manufacture (Regula et al., 2014).

Table 2.3 - Classification of various cleaning chemicals (Adapted from Regula et al., 2014)

\begin{tabular}{|c|c|c|c|}
\hline Category & $\begin{array}{c}\text { Primary Foulant } \\
\text { Targeted }\end{array}$ & Typical Chemicals & $\begin{array}{c}\text { Major } \\
\text { Function }\end{array}$ \\
\hline Caustic & $\begin{array}{c}\text { Organic and } \\
\text { microbial }\end{array}$ & $\mathrm{NaOH}$ & $\begin{array}{c}\text { Hydrolysis, } \\
\text { solubilization }\end{array}$ \\
\hline $\begin{array}{c}\text { Oxidants } \\
\text { Disinfectants }\end{array}$ & $\begin{array}{c}\text { Organic and } \\
\text { microbial }\end{array}$ & $\mathrm{NaOCl, \text {Ozone }}$ & $\begin{array}{c}\text { Oxidation, } \\
\text { disinfection }\end{array}$ \\
\hline Acids & $\begin{array}{c}\text { Scales, and } \\
\text { inorganic deposits }\end{array}$ & $\begin{array}{c}\text { Citric acid, nitric acid, } \\
\text { hydrochloric acid }\end{array}$ & Solubilization \\
\hline Chelating agents & $\begin{array}{c}\text { Scales, and } \\
\text { inorganic deposits }\end{array}$ & Citric acid, EDTA & Chelation \\
\hline Surfactants & Biofilms & Surfactants, detergents & $\begin{array}{c}\text { Surface } \\
\text { Conditioning }\end{array}$ \\
\hline
\end{tabular}

Sodium hypochlorite $(\mathrm{NaOCl})$ and sodium hydroxide $(\mathrm{NaOH})$ are the most commonly used chemicals in the maintenance and recovery cleaning of membranes to restore the permeability (Shi et al., 2014). They are used to clean NOM fouling through oxidation, hydrolysis, and solubilization. Oxidation by $\mathrm{NaOCl}$ degrades the $\mathrm{NOM}$ functional groups to carboxyl and aldehyde groups, which make them more susceptible to removal (Wang X. et al., 2018). On the other hand, $\mathrm{NaOH}$ encourages dissolution of carboxylic and phenolic functional groups such as protein and polysaccharides (Porcelli and Judd, 2010). The 
following sections provide summaries of chemical cleaning studies of polymeric and ceramic membranes with a focus on $\mathrm{NaOCl}$ and $\mathrm{NaOH}$ for $\mathrm{NOM}$ cleaning.

\subsubsection{Polymeric membranes cleaning}

Studies addressing the impacts of different cleaning parameters of $\mathrm{NaOCl}$ and $\mathrm{NaOH}$ for cleaning polymeric membranes have shown that chemicals concentration, cleaning sequence, and cleaning $\mathrm{pH}$ are important factors that govern the cleaning efficiency (Wang X. et al., 2018; Puspitasari et al., 2010; Ang et al., 2006; Hilal et al., 2005). Research has shown that there is an optimum concentration, above which cleaning efficiency does not improve and at times may, on the contrary, cause deterioration of membrane materials. Nigam et al. (2008) study on cleaning a UF membrane fouled with whey protein concentrate ( $3 \mathrm{wt} \%$ ) found that the membrane flux recovered by $0.2 \%$ (by mass) $\mathrm{NaOH}$ solution was almost two times higher than that by $0.3 \%$ and $0.5 \% \mathrm{NaOH}$ solutions. Puspitasari et al. (2010) reported that the repeated cleaning of PVDF membranes with $0.5 \%$ $\mathrm{NaOCl}$ was as effective as a single clean performed with 1\%. Wang X. et al. (2018) found that increasing chlorine concentration from 100 to $1000 \mathrm{mg} / \mathrm{l}$ of $\mathrm{NaOCl}$ resulted in fast diffusion of chlorine within the fouling layer, thus, higher oxidizing capability and cleaning efficiency. However, it is commonly known that high concentration of chemicals and frequent cleaning can oxidize membrane polymers and cause swelling of membrane fibers, thus, shortening the membrane lifetime (Puspitasari et al., 2010; Arkhangelsky et al., 2007). Moreover, frequent cleaning can cause changes in polymeric membrane functional groups,

physical structure, and surface properties (Wang Q. et al., 2018; Shi et al., 2014). As a 
result, polymeric membranes are typically restricted to chlorine concentration $<500 \mathrm{ppm}$ (Regula et al., 2014; Porcelli and Judd 2010).

Cleaning sequence is known to affect the degree of membrane permeability recovery (Porcelli and Judd, 2010). As a result, the selection of cleaning sequence is determined by the feed water composition and the type of fouling. For example, previous studies on filtration of surface water showed that caustic/acid or oxidant/acid sequence is more effective than the reverse (Liang et al., 2008; Huang et al., 2007; Kimura et al., 2004). Kimura et al., (2004) study showed that the flux recovery of a polymeric membrane fouled by polysaccharides improved with cleaning using $\mathrm{NaOH} / \mathrm{HCl}$ compared to $\mathrm{HCl} / \mathrm{NaOH}$ sequence. This could be explained by the effect of the charge on the membrane surface and foulants following the $\mathrm{NaOH}$ clean, along with swelling of both the foulant layer and the membrane at higher $\mathrm{pH}$. This allows enhanced cleaning by $\mathrm{HCl}$ in the subsequent step (Kimura et al., 2004).

Cleaning $\mathrm{pH}$ is another important factor that influence the cleaning efficiency of different chemicals. Ang et al. (2006) study on EDTA and sodium dodecyl sulfate (SDS) cleaning of RO membranes fouled with alginate calcium complexes showed that the cleaning efficiency increased noticeably from 25 to $44 \%$ with increasing $\mathrm{pH}$ from 4.9 to 11.0 for EDTA, while there was only a slight increase for SDS at higher $\mathrm{pH}$. For EDTA at pH 11.0, the alginate gel layer was broken down relatively more easily compared to lower $\mathrm{pH}$, whereas no improvement in SDS cleaning at higher pH. Wang X. et al., (2018) found that increasing $\mathrm{NaOCl} \mathrm{pH}$ from 5.0 to 11.0 resulted in fast diffusion of chlorine within the 
fouling layer, thus, higher oxidizing capability and cleaning efficiency. However, high cleaning $\mathrm{pH}$ ages polymeric membranes faster by changing membrane physical structure and surface properties. (Wang Q. et al., 2018; Regula et al., 2014). Thus, polymeric membranes are typically restricted to $\mathrm{pH} 11$ or lower (Regula et al., 2014).

\subsubsection{Ceramic membranes cleaning}

Ceramic membranes have many advantages over polymeric membranes as they are mechanically, thermally, and chemically stable (Kim et al., 2008). Most importantly, ceramic membranes have superior chemical resistivity allowing much more aggressive cleaning approaches (i.e. $\mathrm{pH}>11$ and high chemicals concentration) without risk of damaging membrane integrity (Lee et al., 2013). Moreover, utilization of high $\mathrm{pH}$ cleaning (i.e. $\mathrm{pH} 12$ or higher) in a single or stepwise approach with the $\mathrm{NaOCl}$ and $\mathrm{NaOH}$ solutions can be beneficial for controlling irreversible fouling of ceramic membranes for drinking water treatment applications. This is usually not available for polymeric membranes as they are less resistant to high $\mathrm{pH}$ and/or repetitive cleaning (Wang Q. et al., 2018). Few studies exist that investigate chemical cleaning performance of ceramic membranes most of which are related to ceramic membranes application in oil, gas, and food processing industry. For example, Yin et al., (2013) found that combination of $\mathrm{NaOCl}$ and $\mathrm{NaOH}$ followed by $\mathrm{HNO}_{3}$ was effective in cleaning ceramic UF membrane applied to desulfurization wastewater. This can be supported by Li et al., (2018) study on cleaning of ceramic UF membrane fouled by limed sugarcane juice which showed that more than $96 \%$ of flux recovery was achieved using a mixture of $1.0 \% \mathrm{NaOH}$ and $0.5 \% \mathrm{NaOCl}$, followed by $0.5 \% \mathrm{HNO}_{3}$ solution. 
In addition, ceramic membranes are ozone resistant, thus, the oxidation of NOM on the ceramic membrane surface can be expected, because of properties of metal oxides that form the separation layer of the membrane (Sanchez-Polo et al., 2006). The use of ozone with ceramic membranes for the treatment of natural waters has shown to reduce membrane fouling (Zhang et al., 2013; Kim et al., 2008). Ozone can oxidize NOM through a direct molecular ozone attack as well as an indirect one through: free radicals formation resulting from ozone decomposition in the bulk phase, ozone oxidation of NOM, or ozone reaction with the ceramic membrane surface (Regula et al., 2014; Van Geluwe et al., 2011). Karnik et al. (2005) found that membrane flux improved by $65 \%-90 \%$ when the ceramic membrane was combined with ozonation. Other studies such as Sartor et al. (2008) and Zhang et al. (2013), reported that in situ ozonation transformed organic molecules into smaller ones and more hydrophilic, thus, less fouling. It should be noted that in the above studies, ozone is dosed in a pre-ozonation mode or with backwash step, or continuously injected into the membrane tank. However, limited research exists that investigate alternative ozone CIP cleaning regimes with respect to NOM fouling control for ceramic membranes. Furthermore, the ability to use ozone CIP with ceramic membranes may prove to have superior fouling removal versus traditional cleaning chemicals (e.g., $\mathrm{NaOCl}$ and $\mathrm{NaOH})$ that are utilized with polymeric systems. Optimization of membrane cleaning protocols requires in depth understanding of the complex interactions between the foulant and the membrane. A more systematic approach is required to study the various aspects of fouling control. In addition, it is important to consider the economic impact of cleaning procedures, including the costs of the cleaning process itself along with the effect of the procedures on membrane lifetime and efficiency. While the majority of the current 
research on chemical cleaning used polymeric membranes, little to no research exists on cleaning of ceramic membranes for drinking water treatment. Research which examines CIP methodologies with different NOM fractions is required to increase operational understanding of cleaning regimes that control irreversible fouling of ceramic membranes.

\subsection{Summary of Research Needs}

Fouling is the major obstacle for successful implementation of membrane processes for drinking water treatment. As discussed in this chapter, a great deal of effort has been made to understand membrane fouling in terms of mechanism, nature, prediction, and prevention. However, inconsistent results have been reported regarding the primary NOM contributor of membrane fouling and still remains to be determined by further research. The composition of NOM changes with locations, thus, it is of interest to study the impact of various NOM fractions have on membrane fouling.

The application of fouling indices such as the SDI, $\mathrm{MFI}_{0.45}$, and MFI-UF as methods to assess NOM fouling is an area that has been frequently questioned in terms of reliability and precision. These methods are performed according to documented testing procedures to estimate an index value that is used as an indicator of the severity of fouling and not considering the typical operation of full-scale membrane systems. The ability of the MFIUF to predict NOM fouling under changes of membrane process conditions has received little attention, which is necessary for the successful implementation of MFI-UF for NOM fouling prediction. 
Fouling models based on the blocking law assume that only one fouling mechanism is controlling, this is probably not realistic. Furthermore, this approach can only be applied to one filtration cycle, while membrane systems operate with multiple filtration and backwash cycles and thus the fouling mechanisms may change from the initial cycles to subsequent cycles. The UMFI method is useful in that it does assume different fouling mechanism and quantify fouling based on foulants response to physical and chemical cleaning operations. However, different approaches in fouling assessment may suggest that direct comparison lack context. The applicability of the UMFI to be effectively used with the MFI-UF in assessing NOM fouling under changes in membrane process conditions, such as water temperature, with both polymeric and ceramic membranes needs to be clarified to help control fouling.

Ceramic membranes are an emerging technology for water treatment. Research with ceramic membranes in a drinking water context is severely limited, however, due to the potential advantages of ceramic membranes with regards to cleaning they are expected to grow in use over the next decade. It is necessary to investigate the NOM fouling characteristics of ceramic membranes to better clarify if and how it fouls differently than the more common polymeric membranes in drinking water treatment. The identification of the dominant contributing resistances would influence the cleaning strategy for different membrane materials. In addition, the robustness of ceramic membranes against rigorous chemical cleaning conditions (i.e. cleaning $\mathrm{pH} 12$, ozone, etc.) may prove to be useful in controlling irreversible fouling of ceramic membranes and may be the key advantage to ease of integration of ceramic membranes into drinking water applications. 


\title{
Chapter 3
}

\section{Application of MFI-UF Fouling Index with NOM Fouling under Various Operating Conditions}

\author{
Mohammad T. Alresheedi ${ }^{*}$ and Onita D. Basu* \\ *Department of Civil and Environmental Engineering, Carleton University, 1125 Colonel \\ By Drive, Ottawa, ON, K1S 5B6, Canada. \\ Published: Journal of Desalination and Water Treatment
}

\begin{abstract}
Fouling research with polymeric membranes has demonstrated that various NOM fractions contribute differentially to membrane fouling behavior. However, limited studies exist analyzing the sensitivity of the MFI-UF to be used as a tool to differentiate NOM fouling components. The results here indicate that MFI-UF is a suitable tool for assessing NOM fouling. Specifically, NOM fouling potential was in the order of organic proteins (as BSA), polymers (alginate), and humic acid, respectively. Further, a mixed solution containing BSA, alginate and humic acid fouled similarly to the BSA solution indicating the high fouling potential of organic proteins in membrane systems. The MFI-UF value was found to increase by $>30 \%$ with increasing pressure (1-3 bar) and decreasing temperature (35-5 $\left.{ }^{\circ} \mathrm{C}\right)$. The filtered water volume was found to correlate with the MFI-UF values indicating the dependency of the method on testing conditions. Incorporating water viscosity and pressure values against normalized conditions $\left(20^{\circ} \mathrm{C}\right.$ and 2 bar) with the standard MFI-UF equation was found to be useful to estimate MFI-UF values at variable operating
\end{abstract}


conditions, thus, enhances the potential application range of MFI-UF as a fouling index for NOM.

Keywords: MFI-UF; Humic acid; BSA; Sodium alginate; Temperature

\subsection{Introduction}

Ultrafiltration (UF) is considered a promising process for drinking water production because of its easy automation, compactness, and high removal rate of various water contaminants (Gao et al., 2011). However, one of the main challenges in membrane processes is fouling which decreases membrane efficiency in terms of flux, increases pressure requirements and cleaning frequency, and ultimately decreases the lifetime of the membrane unit (Peiris and Jaklewicz, 2013). Previous studies on membrane fouling have provided valuable insights on the major foulants during the filtration of natural waters. This includes natural organic matter (NOM), inorganic substances, particulate/colloids matter, and microbiological compounds (Guo et al., 2012; Betancourt and Rose, 2004). Among these foulants, membrane fouling by NOM is the major concern in membrane processes to treat surface water (Guo et al., 2012). The major NOM fractions that have been linked to membrane fouling were identified as humic acids, proteins, and polysaccharides like substances (Zularisam et al., 2011; Katsoufidou et al., 2010; Zazouli et al., 2010). Therefore, prevention and control of NOM fouling is essential for successful operation of membrane systems.

Over the past decades, researchers have focused their effort to develop fouling prediction methods such as the silt density index (SDI) and modified fouling index (MFI), to assess 
the fouling potential of membrane feed with a goal of reducing the fouling problem. These methods are indirect estimate of fouling potential making use of microfiltration (MF) filters with pores of $0.45 \mu \mathrm{m}$. The simplicity of these tests has led to their popularity; however, there are growing doubts about the accuracy of these tests for fouling prediction. Membranes manufacturers are frequently questioned that the filtered water does not meet the SDI/MFI values reported by the manufacturer nor the treatment requirement (Nagel et al., 1987). These observations could be attributed to the lack of agreement between fouling indices testing conditions and membrane systems. In fact, it has been proven that particles smaller than $0.45 \mu \mathrm{m}$ are responsible for fouling of the membrane surface (Boerlage et al., 2002; Schippers and Verdoauw, 1980). Therefore, Boerlage et al (2002) developed the MFI-UF, making use of ultrafiltration (UF) membranes to count for particles $<0.45 \mu \mathrm{m}$. The MFI-UF has been tested for seawater and used at constant pressure and constant flux (Boerlage et al., 2004; Boerlage et al., 2002). Feed water having an MFI-UF $<3000 \mathrm{~s} / \mathrm{L}^{2}$ is equivalent to SDI $<3 \% / \mathrm{min}$, which is acceptable for membrane feed (Boerlage et al., 2002).

The utilization of the MFI-UF for assessing the fouling propensity has been carried out in recent studies. For example, Rodriguez et al. (2015) and Jeong and Vigneswaran. (2015) used the MFI-UF to assess particulate and biofouling potential for RO systems. Moreover, MFI-UF was applied to assess fouling potential of different species of bloom forming algae in marine and freshwater sources (Villacorte et al., 2015). Other studies (Ju and Hong., 2015; Taheri et al., 2013), investigated the influence of inorganic silica and calcium colloids on the MFI-UF fouling index for seawater RO systems. The MFI-UF showed 
promising results with regards to assessing fouling, however, to date, the applicability of the MFI-UF testing to predict NOM fouling for low pressure membranes has received little attention.

The standardized MFI-UF test is performed under a constant pressure of 2 bar and temperature of $20{ }^{\circ} \mathrm{C}$. However, operating pressures and water temperatures change seasonally and over filtration cycles in membrane systems, thus developing a model away from standard conditions may extend its useful applicability. Moreover, to date, the ability of the MFI-UF to predict NOM fouling under changes of membrane process conditions has received little attention, which is necessary for the successful implementation of MFI-UF for NOM fouling prediction. Therefore, the objectives of this research are to evaluate the MFI-UF method as a tool to predict fouling of various NOM fractions commonly found in surface water sources. Further to assess the MFI-UF method under a range of pressure and temperature conditions in low pressure membrane systems and to propose empirical models that can be used to extend its useful application range for fouling studies.

\subsection{Materials and Methods}

\subsubsection{Experimental setup and approach}

The MFI-UF setup consisted of a digital gear pump (Cole Parmer: Drive No. 75211-30, Head No. 07003-04); a ball valve (Cole Parmer No. 01377-18); a pressure relief valve (Aquatrol No. 3ETU4), and a pressure regulator with gauge (Veolia No. LA512). PAN UF, hollow fiber, inside-out membrane module (Pall Corp.) was used and its characteristics are presented in Table 3.1. This UF membrane was proposed by Boerlage et al. (2002). High- 
capacity precision top loading balance (Adam Equipment NBL8201e) to measure the permeate volume. A schematic representation of the MFI-UF setup is shown in Figure 3.1.

Table 3.1 - MFI-UF membranes characteristics

\begin{tabular}{cc}
\hline Membrane Type & UF - 13 kDa \\
Membrane Materials & PAN hollow fiber \\
Configuration & Inside-out \\
\hline Fiber ID & $0.8 \mathrm{~mm}$ \\
Fiber OD & $1.4 \mathrm{~mm}$ \\
Number of Fiber & 400 \\
\hline Membrane Area & $0.2 \mathrm{~m}^{2}$ \\
Module Length & $347 \mathrm{~mm}$ \\
Module Diameter & $42 \mathrm{~mm}$ \\
\hline
\end{tabular}

The MFI-UF (constant pressure) was performed according to method described by Boerlage et al. (2002). Feed water was filtered through the UF membrane under dead-end mode, constant pressure while monitoring flux decline during the test. Permeate was collected in a tank set on the electronic balance which has an RS 232 interface with a computer in order to acquire permeate weight and filtration time data from the balance. Data were recorded every 1 minute and imported into MS Excel spread sheet with data terminal software (TeraTerm). The MFI-UF was then calculated using Equation 3.1 (Boerlage et al., 2002).

$\frac{\mathrm{t}}{\mathrm{V}}=\frac{\mu \mathrm{R}_{\mathrm{m}}}{\Delta \mathrm{PA}}+\left(\frac{\mu \mathrm{C}_{\mathrm{b}} \alpha}{2 \Delta \mathrm{PA}^{2}}\right) \mathrm{V}=\frac{\mu \mathrm{R}_{\mathrm{m}}}{\Delta \mathrm{PA}}+\mathrm{MFI}-\mathrm{UF} \exp (\mathrm{V})$ Equation (3.1)

Which can be rewritten as

$$
\mathrm{MFI}-\mathrm{UF}_{\exp }=\frac{\mathrm{d}\left(\frac{\mathrm{t}}{\mathrm{v}}\right)}{\mathrm{dV}} \quad \text { Equation (3.2) }
$$


Where $\mathrm{t}$ is the filtration time (s); $\mathrm{V}$ is permeate produced $\left(\mathrm{m}^{3}\right) ; \Delta \mathrm{P}$ is the pressure (bar); $\mu$ is the viscosity of water $(\mathrm{kg} / \mathrm{m} \mathrm{s})$; $A$ is the membrane area $\left(\mathrm{m}^{2}\right) ; \mathrm{R}_{\mathrm{m}}$ is the membrane resistance $\left(\mathrm{m}^{-1}\right) ; \alpha$ is the specific cake resistance $(\mathrm{m} / \mathrm{kg})$; and $\mathrm{C}_{\mathrm{b}}$ is the particle concentration $\left(\mathrm{kg} / \mathrm{m}^{3}\right)$. The MFI-UF experimental $\left(M F I-U F_{\text {exp }}\right)$ represents the slope of the linear portion of the t/V versus $V$ in Equation 2 (Boerlage et al., 2002). The slope of two data points from the $\mathrm{t} / \mathrm{V}$ and $\mathrm{V}$ measurements was calculated over time and used to graph the MFI-UF as a function of filtration time (Boerlage et al., 2003; Boerlage et al., 2002). Boerlage et al. (2002) study showed that the MFI-UF value increases sharply with time during first few hours of filtration due to pore blocking then stabilizes afterwards (i.e. remains unchanged for several hours). The stabilization of the MFI-UF value was believed to be due to the occurrence of cake filtration in which the rate of increase in the filtered water volume remains constant with time. Thus, in this study, the final MFI-UF was determined by averaging the MFI-UF data in the most stable region of the MFI-UF graph (Boerlage et al., 2002). To examine the effect of testing conditions on the MFI-UF measurements, experiments were carried out under various pressure and temperature conditions. The operating pressure was varied between 1,2 , and 3 bar $(15,30$, and 45 psi) whereas water temperature was varied between $5,10,20,30$, and $35^{\circ} \mathrm{C}$. 
BV: Ball Valve P: Pressure Regulator PR: Pressure Relief Valve G: Gauge
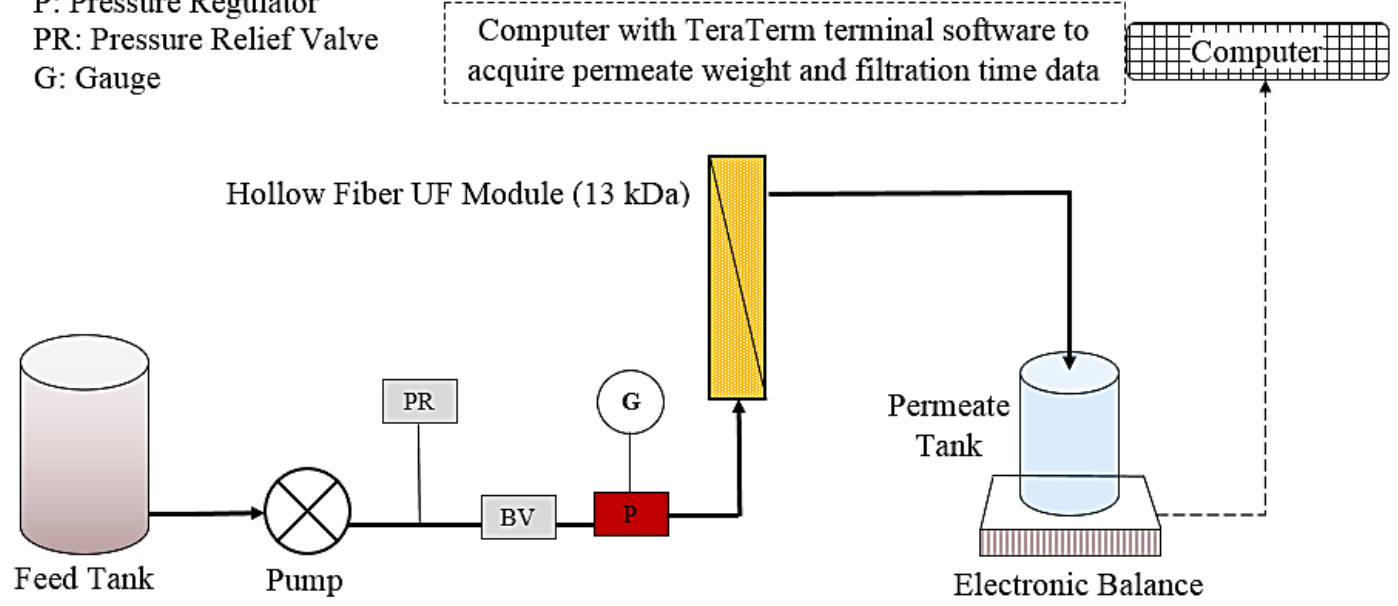

Figure 3.1- A schematic representation of the MFI-UF setup

The UF membrane was cleaned after each MFI-UF experiment. The membrane was first rinsed with DI water for approximately 30 minutes followed by chemical cleaning using sodium hypochlorite $(200 \mathrm{ppm})$, which was recirculated for 60 minutes at ambient temperature followed by citric acid clean (1\%). Clean water flux was measured before any experiment and calculated using Equation 3.3 (Crittenden et al., 2005) corrected to temperature of $20^{\circ} \mathrm{C}$ and pressure of 2 bar (recommended by the manufacture). This was important to ensure good stability of the UF membrane before any experiment.

$\mathrm{J}_{20}{ }^{\circ} \mathrm{C}=\mathrm{J}_{\mathrm{m}}(1.03)^{\mathrm{T}} 20^{\circ} \mathrm{C}^{-\mathrm{T}} \mathrm{m}$

Equation (3.3)

\subsubsection{Feed solutions}

Four different synthetic feed water solutions used in this study. Humic acids $(2.5 \mathrm{mgC} / \mathrm{L})$, a protein (bovine serum albumin, BSA, $2.5 \mathrm{mgC} / \mathrm{L}$ ), a polysaccharide (sodium alginate, 2.5 
$\mathrm{mgC} / \mathrm{L})$, and a mixture of the three NOM models $(0.83 \mathrm{mgC} / \mathrm{L} /$ each NOM model, total of $2.5 \mathrm{mgC} / \mathrm{L}$ ), were used as model NOM foulants. These NOM models are commonly found in surface water and can significantly contribute to membrane fouling (Hashino et al., 2011; Katsoufidou et al., 2010). All model substances were purchased from Sigma Aldrich. A moderate hardness and alkalinity of $75 \mathrm{mg} / \mathrm{L}$ calcium carbonate $\left(\mathrm{CaCO}_{3}\right)$ and a low level of turbidity ( $5 \mathrm{NTU})$ as kaolin clay particles were included in the synthetic water matric to represent the more complex conditions of a surface water source. Previous research on fouling during surface water treatment have reported that calcium will cause the NOM particles to be less negative due to double layer compression and reduction of the electrostatic repulsion forces between particles and membrane surface, thus, increasing NOM fouling rate (Zularisam et al., 2011; Jermann et al., 2008). In addition, the presence of kaolin clay particles during filtration was reported to act as an adsorbent of NOM, thus, increasing NOM adsorption onto the membrane surface and consequently resulting in high flux decline (Jermann et al., 2008). Therefore, in this study, it was necessary to examine NOM-calcium-particles interactions in water during MFI-UF testing. This is of particular importance because the raw water characteristics may vary significantly with respect to the NOM, calcium, and particle concentrations, thus, impacting fouling estimation.

Feed solutions were prepared using DI water and were mixed using a magnetic stirrer oneday prior any experiment to ensure that materials were dissolved completely. Feed water was continuously mixed using a VWR dual speed mixer to ensure homogeneous water conditions throughout the experiment. The feed tank was insulated to maintain constant temperature throughout the testing period. An immersion heater (Cole Parmer) and a 
compact chiller (LM series, Polyscience) were used to adjust the water temperature as required. Temperature and $\mathrm{pH}$ were monitored continuously using HACH (cat.no. 5825800) HQd Field Case equipment. The $\mathrm{pH}$ of the feed was maintained around $7.5 \pm 0.10$.

\subsubsection{Analysis}

\subsubsection{Molecular weight fractionation of NOM}

The molecular weight distribution of the feed solutions, which are comprised of the respective NOM components, kaolin clay, and calcium, was determined by ultrafiltration (UF) fractionation method using a $400 \mathrm{~mL}$ UF stirred cell (model 8400, Amicon Inc.) and five hydrophilic regenerated cellulose membranes (Millipore, Bradford MA) with different molecular weight cut offs (MWCOs) of 1,000, 5,000, 10,000, 30,000, and 100,000 Daltons. $2 \mathrm{~L}$ of feed water solutions were prepared for the fractionation. The fractionation was performed sequentially by passing $400 \mathrm{~mL}$ of sample through the membrane with the highest MWCO to that with the lowest MWCO. Samples from permeate and retentate of particular membrane were collected and total organic carbon (TOC) was analyzed using a Tekmar Dohrmann, Phoenix 8000 TOC analyzer. Excess permeate produced by each fractionation step was used as the feed to the next membrane with a lesser MWCO. The UF fractionation method used in this study is described in detail by (Mosqueda-Jimenez et al., 2003; Kitis et al., 2002). The TOC concentration of a particular molecular weight fraction was calculated by subtracting the TOC concentration of the filtrate from one membrane from the TOC concentration of the filtrate from the membrane of the next larger nominal MWCO. After the fractionation experiments, membranes were rinsed with MilliQ water for 60 minutes changing the water three times (i.e., every 20 minutes) and stored 
in $10 \%$ by volume ethanol/water solution and kept in refrigerator at $4{ }^{\circ} \mathrm{C}$ for later use. Prior to use, the membranes were rinsed several times after which Milli-Q water was filtered through them for three times.

\subsubsection{MFI-UF data analysis}

One-way ANOVA (using the F-test) on the data collected to determine the significance of the MFI-UF measured at different pressure and temperature conditions. The mean difference between conditions was considered to be significant at a $p$-value of $\leq 0.05$. Post ANOVA test (Tukey HSD test) was then performed to determine pair-wise comparisons between the various testing conditions.

To differentiate between pore blocking and cake filtration mechanisms during the MFI-UF testing, the combined model proposed by Ho and Zydney (2000) was used to analyze the filtration data. Four major fouling mechanisms are described as complete pore blocking (n $=2)$, standard blocking $(\mathrm{n}=1.5)$, intermediate blocking $(\mathrm{n}=1)$ and cake filtration $(\mathrm{n}=0)$. A generalized equation describing the mechanisms is shown in Equation 3.4.

$\frac{\mathrm{d}^{2} \mathrm{t}}{\mathrm{dV}^{2}}=\mathrm{k}\left(\frac{\mathrm{dt}}{\mathrm{dV}}\right)^{\mathrm{n}}$

Equation (3.4)

Which can be rewritten as

$\log \left(\frac{\mathrm{d}^{2} \mathrm{t}}{\mathrm{dV^{2 }}}=\log \mathrm{k}+\mathrm{n} \log \left(\frac{\mathrm{dt}}{\mathrm{dV}}\right)\right.$

Equation (3.5) 
In which $\mathrm{t}$ is time (s), $\mathrm{V}$ is volume (L), $\mathrm{k}$ is blocking law filtration coefficient (units vary depending on $\mathrm{n}$ ), and $\mathrm{n}$ is blocking law filtration exponent (unitless).

\subsubsection{Modelling of the MFI-UF}

Operating pressure and water temperature change seasonally and over filtration cycle time periods in membrane systems, thus, it may not be practical to alter an MFI-UF setup for different operating pressures or water temperatures. In this study, the MFI-UF exp calculated from the slope of the linear portion of the $t / \mathrm{V}$ versus $\mathrm{V}$ graphs (i.e. Equation 3.2) was normalized to $\left(\mathrm{MFI}-\mathrm{UF}_{\mathrm{nor}}\right)$ by including pressure and viscosity correction terms as shown in Equation 3.6. This was important to determine the ability of the MFI-UF to predict fouling conditions away from the standard reference conditions. The normalized model is derived from the modified fouling index (MF) model, ASTM method (2015). In addition, a general linear regression modelling (GLM) (Equation 3.7), was used to estimate the predicted MFI-UF value $\left(\mathrm{MFI}-\mathrm{UF}_{\mathrm{pr}}\right)$ at different conditions. The MFI-UF $\mathrm{Fr}_{\mathrm{pr}}$ was utilized to establish the influence of the operating pressure and water temperature on the final MFIUF values. The MFI-UF $F_{\text {exp }}$ was then compared to the MFI-UF $F_{\text {nor }}$ and MFI-UF $F_{\text {pr }}$.

$$
\begin{array}{ll}
\text { MFI-UFnor }=\frac{\mu_{20}{ }^{\circ} \mathrm{C}}{\mu_{\mathrm{T}}} \times \frac{\mathrm{P}}{\mathrm{P}_{0}} \times \mathrm{MFI}-\mathrm{UF}_{\mathrm{exp}} & \text { Equation (3.6) } \\
\text { MFI-UFpr }=\beta_{0}+\beta_{1} \text { Pressure }+\beta_{2} \text { Temperature } & \text { Equation (3.7) }
\end{array}
$$

Where: $\mu_{\mathrm{T}}$ and $\mathrm{P}$ are the water viscosity, operating pressure respectively. $\mu_{20}{ }^{\circ} \mathrm{C}, \mathrm{P}_{0}$, are included to normalize the MFI-UF values to the standard reference conditions of pressure 
and temperature (i.e. 2 bar and $20^{\circ} \mathrm{C}$ ). $\beta_{0} \beta_{1}, \beta_{2}$ are the intercept and regression coefficients related to the effect of pressure and temperature respectively.

\subsection{Results and Discussion}

\subsubsection{Molecular weight distribution of feed solutions}

The molecular weight distribution analysis of feed solutions (refer to Figure 3.2) indicate that the majority of the particles in the feedwater are greater than $30 \mathrm{kDa}$, and a higher percentage of particles $(>70 \%)$ are expected to be retained by the MFI UF $13 \mathrm{kDa}$ membrane. Feed solutions containing BSA and NOM mixture showed higher percentage of particles in the range of $>30 \mathrm{kDa}$, thus, they are expected to cause pore blocking and have a higher MFI-UF value than the other organic fractions. The MW distribution of the humic acid and alginate feed solutions is approximately similar which indicates that the differences in fouling would be highly influenced by their chemical and material properties versus size alone. However, all four model solutions are expected to foul the membranes by cake formation, pore blockage, or a combination of both as commonly occurs in a UF membrane process. 


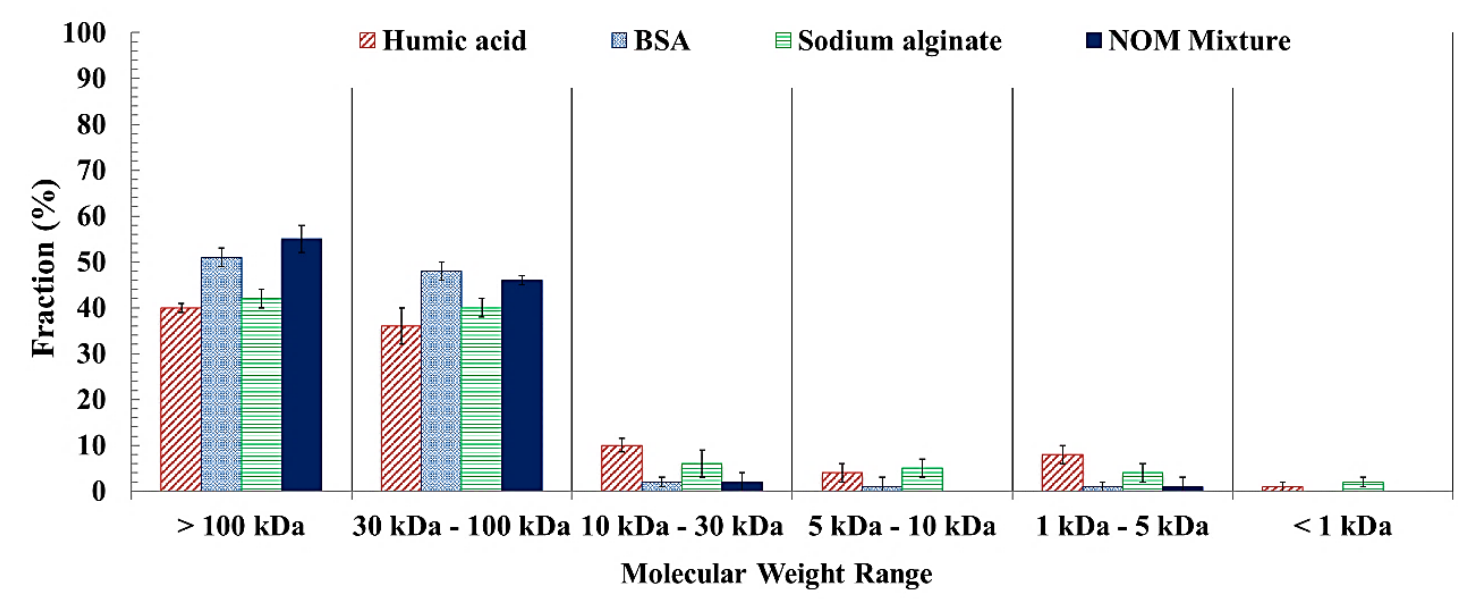

Figure 3.2- Molecular weight distribution of feed water solutions

\subsubsection{Effect of variation of operating pressure and water temperature on the MFI-}

\section{UF measurement to predict NOM fouling}

\subsubsection{MFI-UF measurement under variable operating pressure}

In order to determine the MFI-UF values for each testing condition, the slope of two data points from the $t / \mathrm{V}$ and $\mathrm{V}$ measurements are calculated over time and used to graph the MFI-UF as a function of filtration time (Boerlage et al., 2003; Boerlage et al., 2002). Figure 3.3 is an example of the MFI-UF curves as a function of time under different operating pressures. It can be clearly seen that the MFI-UF graph in Figures 3.3A and 3.3C is divided into two regions: at the beginning of filtration (the first region), the MFI-UF increases sharply during the first 1 to 1.5 hours of filtration. This sharp increase in the MFI-UF value is due to pore blocking as indicated by Boerlage et al. (2002). The blocking law exponent (n) values were determined for each testing condition by plotting the $\log d^{2} t / d V^{2} v s$. $\log$ $\mathrm{dt} / \mathrm{dV}$ (Figures 3.3B and 3.3D as an example) which range from 1.431 to 1.833 and from 1.772 to 2.040 for 1 bar and 3 bars, respectively. The $n$ values are very close to $1.5-2$ 
indicating the occurrence of standard and complete pore blocking mechanism within the first 1-1.5 hours of filtration. After 2.5-3 hours of filtration, the MFI-UF value rate change slows and then stabilizes for the duration of the test. The stabilization of the MFI-UF value is related to the occurrence of cake filtration (the second region) at which the increase in the accumulated filtered water volume remains constant with time (Boerlage et al., 2002). The $\mathrm{n}$ values for the cake filtration region (refer to Figures 3.3B and 3.3D as an example) are close to 0 which range from 0.014 to 0.082 and from 0.043 to 0.120 for 1 bar and 3 bars respectively. The final MFI-UF values are determined by taking the average of MFI-UF data in the most stable region in the MFI-UF curve. The MFI-UF was found to stabilize (i.e. minimum to no change) after 3-6 hours of filtration.
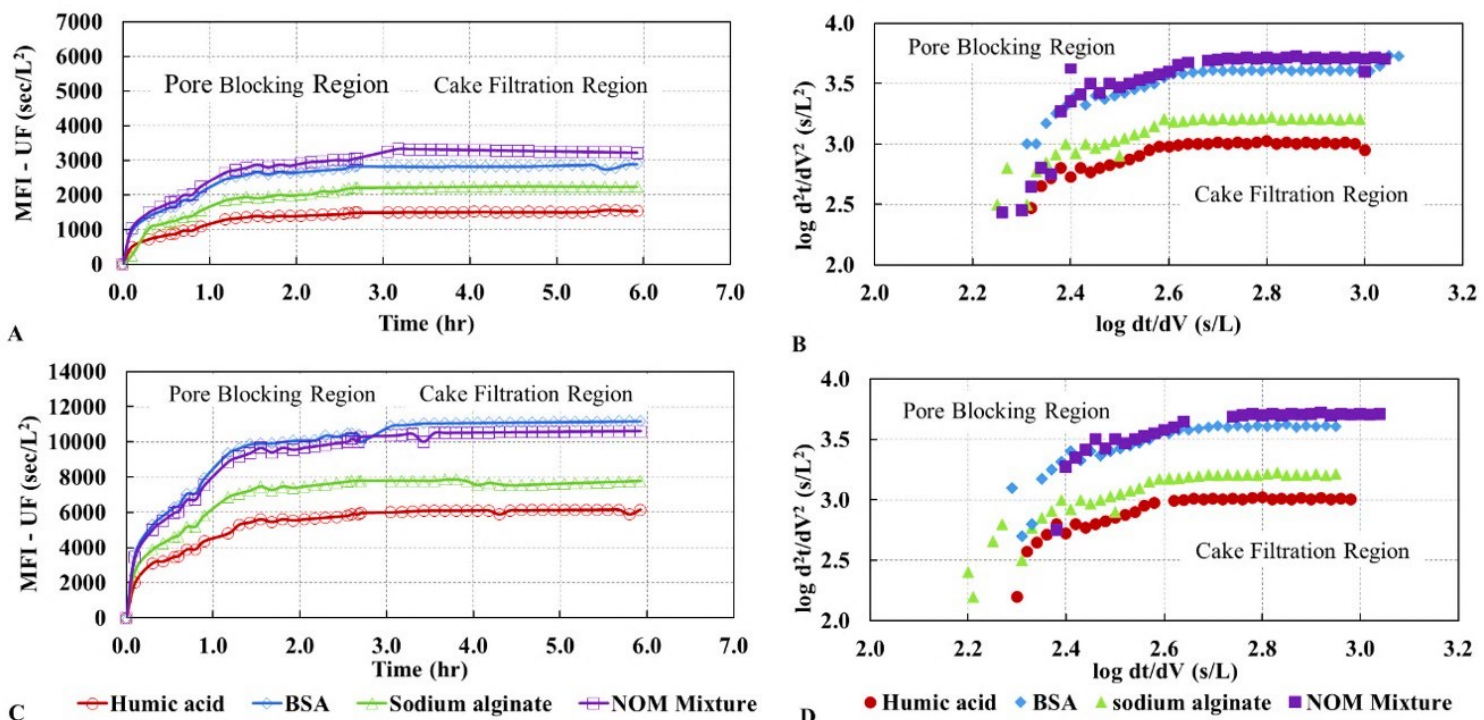

C - Humic acid $\leftarrow$ BSA $\leftarrow$ Sodium alginate $\leftarrow$ NOM Mixture

D

Figure 3.3 - Example of MFI-UF and filtration mechanism graphs for the NOM fractions at different pressure $\left(\mathrm{T}=20^{\circ} \mathrm{C}\right)$
(A) MFI-UF vs time ( $\mathrm{P}=1$ bar); (B) $\mathrm{d}^{2} \mathrm{t} / \mathrm{dV}^{2}$ vs $\mathrm{dt} / \mathrm{dV}$ curves $(\mathrm{P}=1$ bar)
(C) MFI-UF vs time ( $\mathrm{P}=3$ bar); (D) $\mathrm{d}^{2} \mathrm{t} / \mathrm{dV}^{2}$ vs $\mathrm{dt} / \mathrm{dV}$ curves $(\mathrm{P}=3$ bar $)$ 
The MFI-UF exp values (Figure 3.4) increased with increasing operating pressure. At higher operating pressure, more particles were forced towards the membrane forming a denser cake layer with lower porosity and higher resistance (Boerlage et al., 2003), hence, higher MFI-UF value. Moreover, the increase in filtration pressure led to an increase in flux which may cause compression of the cake layer and lead to higher MFI-UF value. The results from a one-way ANOVA (Appendix B, Table B1) indicate that the MFI-UF exp values estimated at different pressure conditions were significant $(\mathrm{p}<0.05)$. Post ANOVA analysis illustrates that the difference between all three pressure categories were also

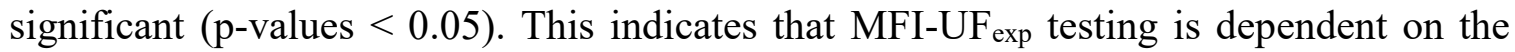
pressure condition and that the fouling rate of the membrane will change as pressure changes in the system; highlighting the importance of assessing fouling index values at both a reference standard condition to compare between general water system but also at operational conditions to increase the predictive power and analysis of the fouling tests.

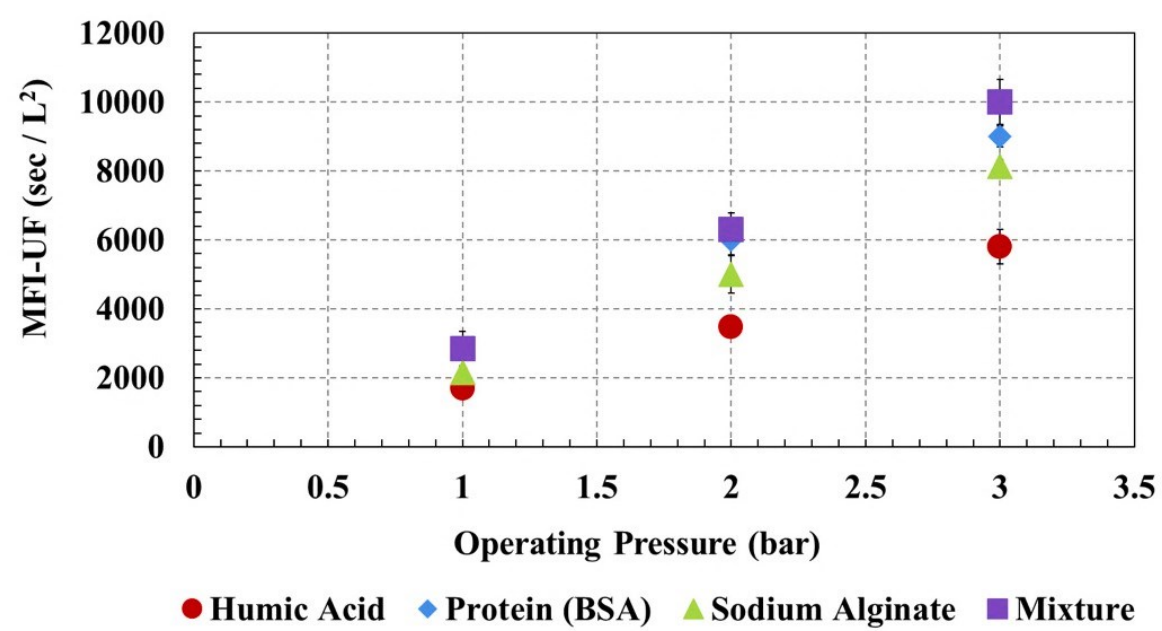

Figure 3.4 - MFI-UF $F_{\text {exp }}$ measured for various NOM fractions at different operating pressure $\left(\mathrm{T}=20^{\circ} \mathrm{C}\right)$ 
Fouling potential of different feed water types predicted by the MFI-UF (constant pressure) was in the order of NOM mixture, BSA (protein), sodium alginate, and humic acid, respectively. The differences between fouling among the four types of NOM could be attributed to the molecular weight distribution (refer to Figure 3.2). Feed water solutions containing BSA and NOM mixture have higher components with molecular weight in the range of 30-100 $\mathrm{kDa}$ and $>100 \mathrm{kDa}$ which was much larger than the MWCO of the UF membrane $(13 \mathrm{kDa})$ and may therefore resulted in more pore blocking leading to higher fouling, thus, higher MFI-UF value compared to sodium alginate and humic acid. These results are also analogous to those by Hashino et al. (2011) and Katsoufidou et al. (2010) using polymeric MF and UF membranes for fouling experiments, in which humic acid had lower fouling rate compared to BSA and alginate under constant pressure filtration experiments. The MFI-UF was able to predict this fouling order which highlights the applicability of the MFI-UF for NOM fouling prediction for low pressure membranes. The NOM mixture resulted in the highest MFI-UF value. This is attributed to the complete pore blocking mechanism of the NOM mixture (n ranges between 1.833 and 2.040), refer to Figures 3.3B and 3.3D, thus, higher final MFI-UF value compared to humic acid, BSA, and alginate alone. This can be supported by molecular weight fractionation (refer to Figure 3.2 ), in which the NOM mixture solution has higher percentage in the size range of $>30$ $\mathrm{kDa}$, thus, it is expected to cause complete pore blocking to the $13 \mathrm{kDa}$ UF membrane and higher MFI-UF. 


\subsubsection{MFI-UF measurement under variable water temperature}

Figure 3.5 is an example of the MFI-UF curves as a function of time under different temperature conditions. It can be clearly seen that the MFI-UF graph in Figures 3.5A and 3.5C is divided into two regions: at the beginning of filtration (the first region), the MFIUF increases sharply due to pore blocking. The blocking law exponent (n) values during the first 1-1.5 hours of filtration (refer to Figures 3.5B and 3.5D as an example) range from 1.800 to 2.060 and from 1.332 to 1.665 for 5 and $35^{\circ} \mathrm{C}$ respectively. The $\mathrm{n}$ values are very close to 2 indicating the occurrence of pore blocking mechanism. After 2.5-3 hours of filtration, the MFI-UF value rate change slows and then stabilizes for the duration of the test indicating the occurrence of cake filtration (the second region) (Boerlage et al., 2002). The $\mathrm{n}$ values for the cake filtration region (refer to Figures 3.5B and 3.5D as an example) range from 0.027 to 0.130 and from 0.011 to 0.066 for 5 and $35^{\circ} \mathrm{C}$, respectively. The final MFI-UF values are determined by taking the average of MFI-UF data in the most stable region in the MFI-UF curve. The MFI-UF was found to stabilize (i.e. minimum to no change) after 3-6 hours of filtration. The MFI-UF graphs at low water temperature $\left(5^{\circ} \mathrm{C}\right)$ follow similar trend as MFI-UF graphs at high pressure (3 bar) and vice versa. 

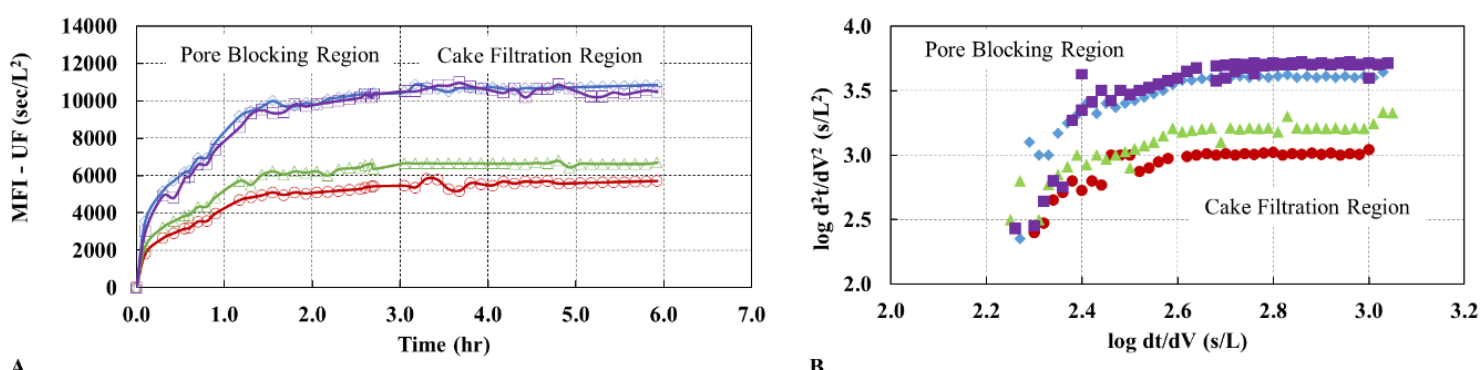

B
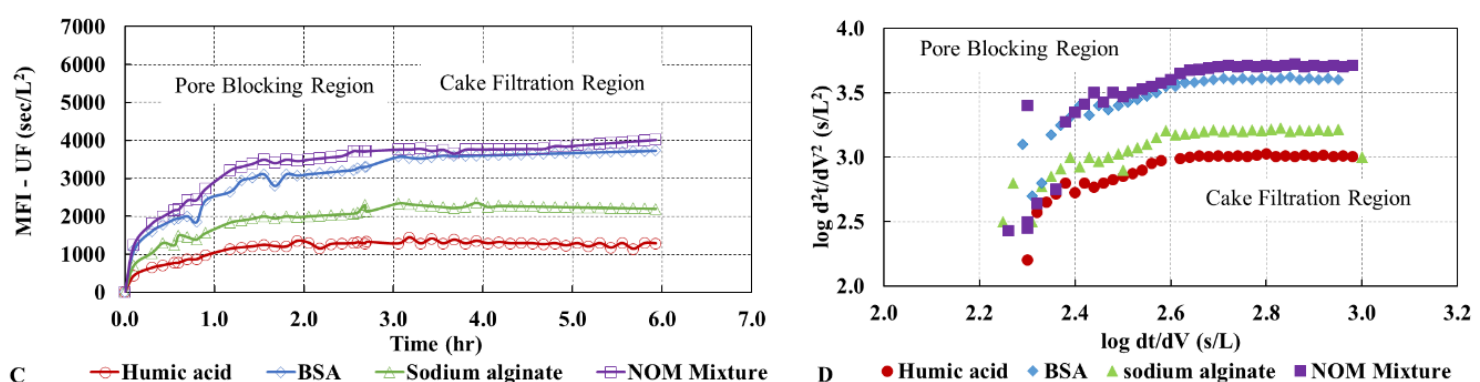

Figure 3.5 - Example of MFI-UF and filtration mechanism graphs for the NOM fractions at different temperature $(\mathrm{P}=1$ bar $)$
(A) MFI-UF vs time $\left(\mathrm{T}=5^{\circ} \mathrm{C}\right) ;(\mathbf{B}) \mathrm{d}^{2} \mathrm{t} / \mathrm{dV}^{2}$ vs dt/dV curves $\left(\mathrm{T}=5^{\circ} \mathrm{C}\right)$
(C) MFI-UF vs time $\left(\mathrm{T}=35^{\circ} \mathrm{C}\right) ;\left(\right.$ D) $\mathrm{d}^{2} \mathrm{t} / \mathrm{dV}^{2}$ vs dt/dV curves $\left(\mathrm{T}=35^{\circ} \mathrm{C}\right)$

The MFI-UF $F_{\exp }$ values (Figure 3.6) decreased with increasing water temperature for all NOM solutions. NOM in cold water temperatures appears to have higher fouling potential compared to that at warmer temperatures. This could be a critical issue in predicting NOM fouling specifically in regions where there is a large seasonal variation in water temperature, as in North America and some parts of Europe. The effect of water temperature on membrane flux was reported to be $3 \%$ per $1{ }^{\circ} \mathrm{C}$ changes in water temperature (Crittrnden et al., 2005). Under constant pressure, the variation in water temperature affected the flux through the UF membrane and ultimately impacted the final MFI-UF measurements. In addition, the decrease in water temperature may cause shrinkage to the cake layer leading to a formation of denser cake with high specific cake resistance, thus, higher MFI-UF (Kertész et al., 2008). The fouling potential order of NOM 
is comparable to that at different pressure conditions. ANOVA analysis (Appendix B, Table B2) shows that the MFI-UF $F_{\text {exp }}$ values estimated at different temperature conditions were significant $(\mathrm{p}<0.05)$. Post ANOVA test illustrates that the difference within groups (e.g. mean of humic acid MFI-UF at 5 vs. $30^{\circ} \mathrm{C}$, etc.) was also significant (p-values $<0.05$ ), demonstrating that the MFI-UF $F_{\text {exp }}$ testing is dependent on the temperature condition.

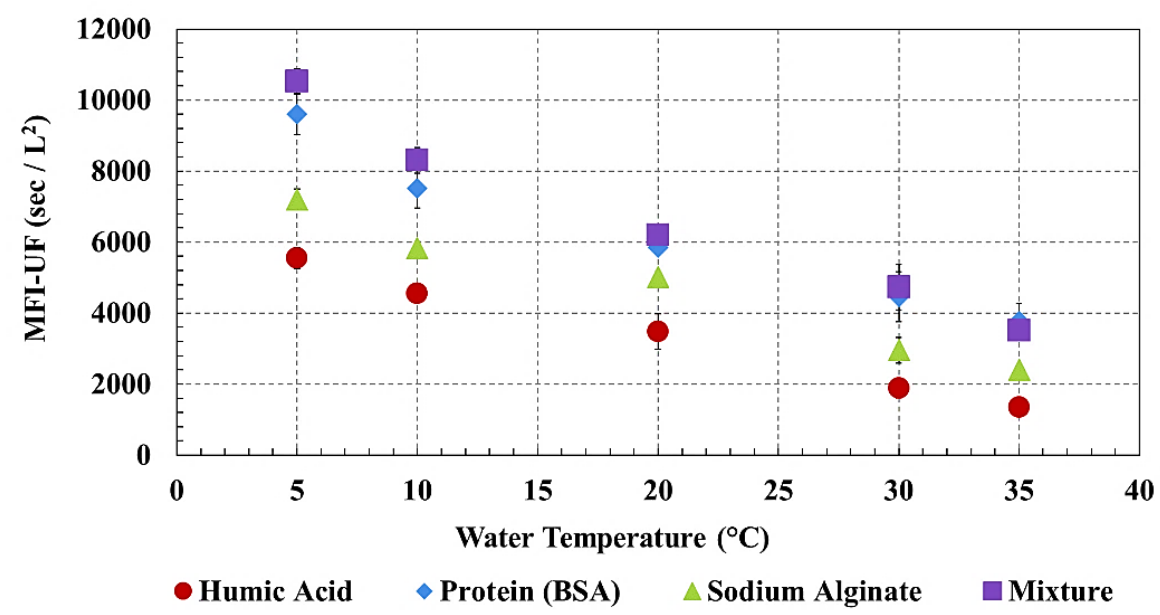

Figure 3.6 - MFI-UF $\mathrm{Fex}_{\mathrm{exp}}$ measured for various NOM fractions at different water temperature $(\mathrm{P}=2$ bar $)$

\subsubsection{MFI-UF surface plots and TOC rejection}

The MFI-UF pr, using GLM (Equation 3.7), was used to establish the influence of the operating pressure and water temperature on the final MFI-UF values. GLM results indicated that both parameters were of significance (Appendix B Table B3). Equations 3.7A-3.7D are the GLM models for different NOM fractions. It should be noted that the $\beta$ values are the standardized regression coefficients and thus, the relative importance of pressure and temperature can be compared regardless of their units. From Equations 3.7A- 
3.7D, it can be clearly seen that for all model NOM, the MFI-UF value increases by $0.652-$ 0.755 units with a unit increase in pressure, holding temperature as a constant. In contrast, the standardized regression coefficient for temperature indicates that for a unit increase in temperature, the MFI-UF decreases by 0.609-0.691 units, holding pressure as a constant. From these models, it is clear that the pressure and temperature both influence the MFI-UF value but in opposing directions.

$$
\begin{aligned}
& \text { MFI-UFpr }=\left(\beta_{0}+\beta_{1} \text { Pressure }+\beta_{2} \text { Temperature }\right) \quad \text { Equation (3.7) } \\
& \text { MFI-UFpr }(\text { Humic })=(1589+0.652 \mathrm{P}-0.623 \mathrm{~T}) \quad \text { Equation }(3.7 \mathrm{~A}) \\
& \text { MFI-UFpr }(\text { Protein })=(3352+0.721 \mathrm{P}-0.691 \mathrm{~T}) \quad \text { Equation }(3.7 \mathrm{~B}) \\
& \text { MFI-UFpr }(\text { Alginate })=(1189+0.755 \mathrm{P}-0.609 \mathrm{~T}) \quad \text { Equation }(3.7 \mathrm{C}) \\
& \text { MFI-UFpr }(\text { Mixture })=(2599+0.739 \mathrm{P}-0.653 \mathrm{~T}) \quad \text { Equation (3.7D) }
\end{aligned}
$$

Figure 3.7 presents 3D surface plots of the MFI-UF simulations as a function of pressure and temperature. The plots illustrates that the MFI-UF value increases with increasing pressure and decreasing temperature. According to Darcy's law, membrane resistance is dependent on both the pressure and water viscosity. The increase in the MFI-UF with pressure increase or temperature decrease could be attributed to resistance increase of the fouling layer, thus, higher MFI-UF values. Proteins (BSA) were found to be particularly sensitivity to increases in pressure, as was the NOM mixture, however this is attributed to the protein component in the mixture as a dominant foulant. 

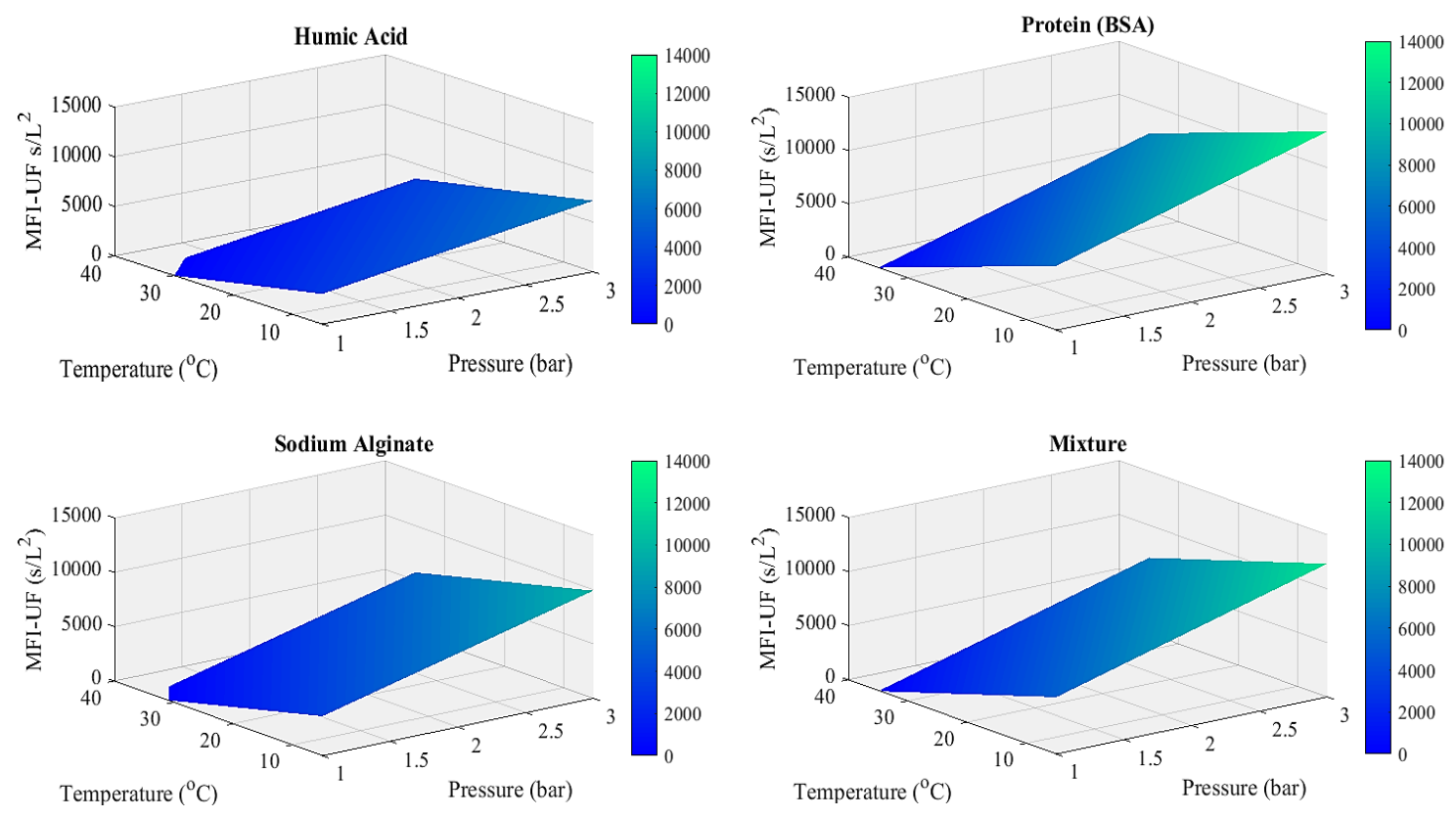

Figure 3.7 - 3D surface plots of the MFI-UF as a function of pressure and temperature

Figure 3.8 illustrates the TOC rejection by the $13 \mathrm{kDa}$ UF membrane versus the MFI-UF fouling index. The TOC measurements were utilized to assess the MFI-UF fouling prediction and to determine any direct correlations between the MFI-UF value determined and the TOC rejected by the UF membrane. This is particularly important as there is no research that has used the TOC measurement as a method of assessing the predictability of the MFI-UF testing.

The TOC rejection of different feed solutions was found to decrease with increasing temperature. At lower temperature $\left(5^{\circ} \mathrm{C}\right),>60 \%$ of TOC was rejected by the UF membrane for all model solutions whereas $<45 \%$ of TOC was rejected at $35^{\circ} \mathrm{C}$. Under all temperature conditions, the highest rejection was for BSA and NOM mixture whereas lower rejection for humic acids and alginate. Therefore, the increase in the MFI-UF value at low 
temperature was related to the high NOM rejection by the UF membrane whereas at high temperature, NOM were passing through the UF membrane to the filtrate side, thus, lower fouling and MFI-UF value.

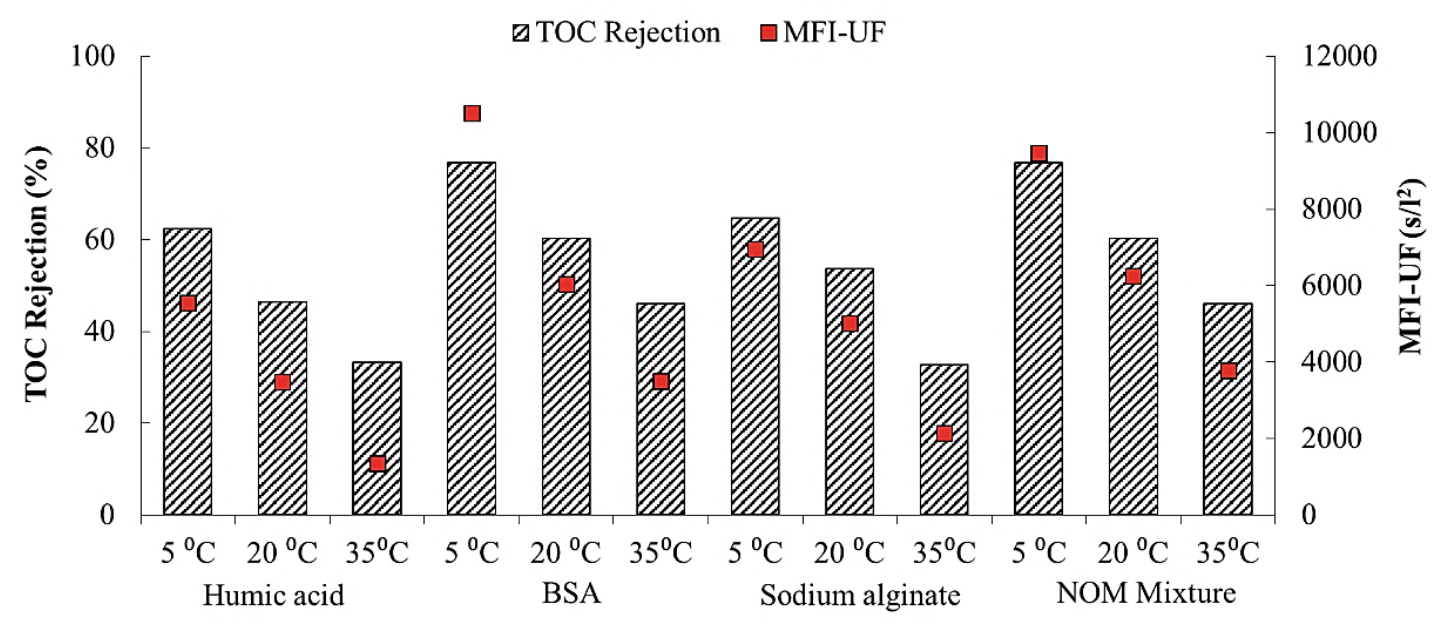

Figure 3.8 - MFI-UF and TOC rejection with water temperature conditions $(\mathrm{P}=2$ bar)

\subsubsection{Modelling of MFI-UF measurements (MFI-UF exp $_{\text {, MFI-UF }}$ nor, MFI-UF pr)}

Operating pressure and water temperature change seasonally and over filtration cycle time periods in membrane systems, thus the current value of the MFI-UF test performed at the standard conditions of 2 bar and $20^{\circ} \mathrm{C}$, while useful for comparing across studies, can overestimate or underestimate the fouling potential of NOM in site specific challenges. An empirical based model that can calculate MFI-UF values at non-standard operating conditions is proposed to help extend it's useful applicate range in terms of NOM fouling assessments.

The MFI-UF value is calculated from relationship between the inverse of the flow rate (t/V) and the permeate volume (V). The obserevd changes in the MFI-UF value at different 
pressure and temperature conditions could be related to the changes in filtration characteristics (e.g., flow, viscosity, etc.) and/or fouling layer properties. Therefore, the

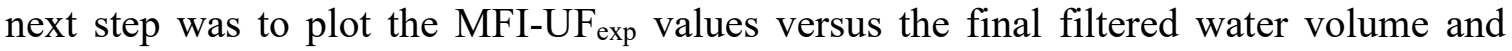
determine if the filtered water volume reflects the fouling conditions observed during testing. Ideally, higher filtered water volume indicates lower MFI-UF value and vice versa. Figure 3.9 presents the filtered water volume as a function of MFI-UF exp $_{\text {for }}$ different NOM solutions. Overall, it can be clearly seen that the water volume decreases with increasing MFI-UFexp value ( $\mathrm{R}^{2}$ between $0.68-0.78$ ) which indicates that both pressure and temperature conditions heavily influence results. The changes in the final filtered water volume is an indicator of the changes in filtration characteristics of NOM water at the different conditions examined.
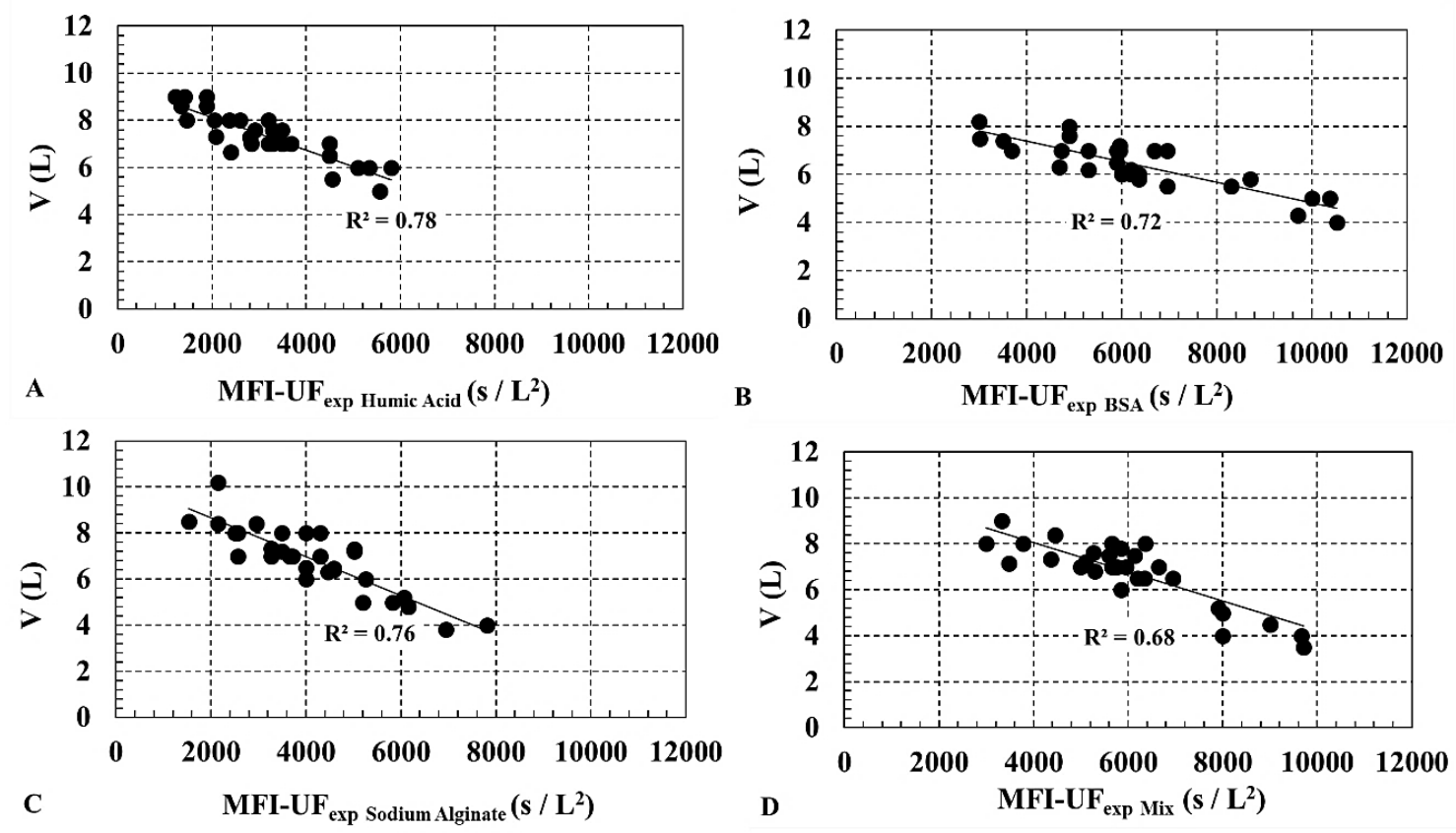

Figure 3.9 - MFI-UF as a function of filtered water volume for the 4 different synthetic solutions (A) Humic Acid, (B) BSA, (C) Sodium Alginate and (D) NOM Mixture 
As both parameters (pressure and temperature) were found to be statistically significant, the next step was to use the model that incorporates water temperature and operating

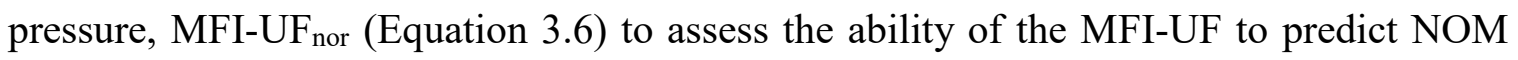
fouling conditions away from the standard reference conditions.

MFI-UF ${ }_{\text {nor }}=\frac{\mu_{20}{ }^{\circ} \mathrm{C}}{\mu_{\mathrm{T}}} \times \frac{\mathrm{P}}{\mathrm{P}_{0}} \times \mathrm{MFI}-\mathrm{UF}_{\mathrm{exp}}$

Equation (3.6)

Figure 3.10 presents a comparison between the MFI-UF $F_{\exp }$ (Equation 3.2), MFI-UF $F_{\text {nor, }}$ (Equation 3.6), and the MFI-UF $F_{\mathrm{pr}}$ (GLM, Equation 3.7). The MFI-UF $\mathrm{F}_{\text {nor }}$ values in Figure 3.10 match the MFI-UF $F_{\exp }$ at the referenced testing conditions (i.e. 2 bar, $20^{\circ} \mathrm{C}$ ) but deviate by $10-15 \%$ at other non-referenced conditions. Therefore, the observed increase or decrease in the MFI-UF $F_{\text {exp }}$ values measured at different conditions was mainly attributed to the fouling tendency of water at specific filtration conditions. Thus, the MFI-UF $F_{\exp }$ values measured at standard conditions can be altered to actual filtration conditions by adjusting the pressure and viscosity terms in the MFI-UF equation. 

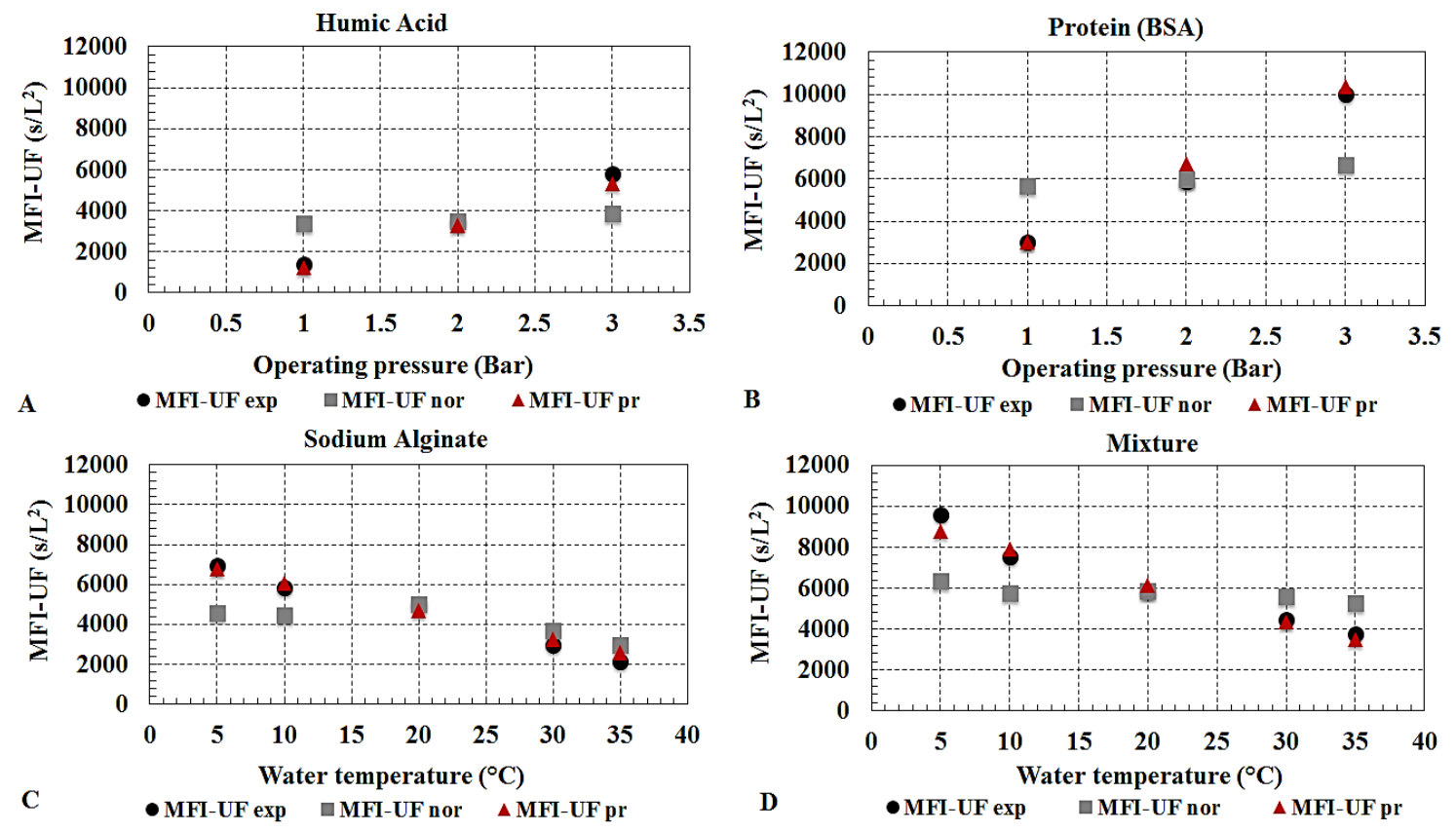

Figure 3.10 - MFI-UF exp, vs. MFI-UF nor vs. MFI-UF $F_{\text {pr }}$ for different model solutions

Thus, from these results, instead of having to design an MFI-UF test for the changes in temperature and pressure, the GLM equations 3.7A-3.7D can be used as preliminary assessment of NOM fouling potential under changes in operational conditions. Future research should investigate the effect of water chemistry (i.e. $\mathrm{pH}$, ionic strength, etc.) on the MFI-UF, as well as advancing the use of MFI-UF as a fouling prediction method for full scale membrane systems.

\subsection{Conclusions}

The presence of NOM in water poses major implications for membrane processes to maintain operational performance. This research investigated the application of the MFIUF method to predict fouling of various NOM fractions under changes in pressure and temperature. The following points summarize the outcomes of this research: 
- The NOM fouling potential order predicted by the MFI-UF exp $_{\text {was }}$ NOM mixture, BSA, alginate, humic acid, respectively. The NOM mixture and BSA solutions exhibited a higher propensity for pore fouling relative to the alginate and humic acid test waters.

- The NOM mixture and BSA were more sensitive to changes in pressure and temperature, as determined by GLM and 3D surface plots, and exhibited higher fouling as temperature decreased and pressure increased.

- The normalization model presented was useful in estimating the MFI-UF values away from standard conditions for the different NOM fractions, which varied between 10$15 \%$ as pressure and temperature changed.

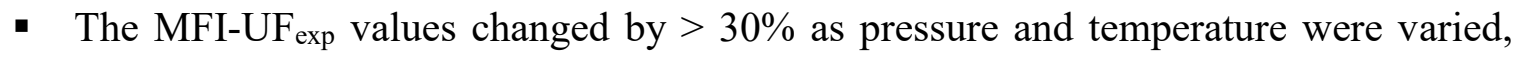
likely due to changes in fouling layer characteristics by cake compression and/or shrinkage.

\subsection{Acknowledgements}

The authors would like to acknowledge the Saudi Arabia Ministry of Education (MOE) and Natural Sciences and Engineering Research Council of Canada (NSERC) for helping fund this research.

\subsection{References}

ASTM International, Designation: (D8002 - 15, 2015). Standard test method for modified fouling index (MFI-0.45) of Water.

Betancourt, W., Rose, J., 2004. Drinking water treatment processes for removal of Cryptosporidium and Giardia. Veterinary Parasitology 126, 219-234. 
Boerlage, S., Kennedy, M., Aniye, M., Abogrean, E., Tarawneh, Z., Schippers, J., 2003. The MFI-UF as a water quality test and monitor. Journal of Membrane Science 211, 271289.

Boerlage, S., Kennedy, M., Dickson, M., El-Hodali, D., Schippers, J., 2002. The modified fouling index using ultrafiltration membranes (MFI-UF): characterisation, filtration mechanisms and proposed reference membrane. Journal of Membrane Science 197, 1-21.

Boerlage, S., Kennedy, M., Tarawneh, Z., Faber, R., Schippers, J., 2004. Development of the MFI-UF in constant flux filtration. Desalination 16, 103-113.

Crittenden, J., Trussell, R., Hand, D., Howe, K., Tchobanoglous, G., 2005. Water treatment: principles and design, 2nd edition, Montgomery Watson Harza Ed, Wiley, New Jersey, USA.

Gao, W., Liang, H., Ma, J., Han, M., Chen, Z., Han, Z., Li, G., 2011. Membrane fouling control in ultrafiltration technology for drinking water production: a review. Desalination $272,1-8$.

Guo, W., Ngo, H., Li, J., 2012. A mini-review on membrane fouling. Bioresources Technology 122, 27-34. 
Hashino, M., Hirami, K., Katagiri, M., Kubota, T., 2011. Effects of three natural organic matter types on cellulose acetate butyrate microfiltration membrane fouling. Journal of Membrane Science 379, 233- 238.

Ho, C., Zydney, A., 2000. A Combined pore blockage and cake filtration model for protein fouling during microfiltration. J. Colloid Interface Science 232, 389-399.

Jeong, S., Vigneswaran, S., 2015. Practical use of standard pore blocking index as an indicator of biofouling potential in seawater desalination. Desalination 365, 8-14.

Jermann, D., Pronk, W., Boller, M., 2008. Mutual influences between natural organic matter and inorganic particles and their combined effect on ultrafiltration membrane fouling. Environmental Science and Technology 42, 9129-9136.

Ju, I., Hong, S., 2015. Multiple MFI measurements for the evaluation of organic fouling in SWRO desalination. Desalination 365, 136-143.

Katsoufidou, K., Sioutopoulos, D., Yiantsios, S., Karabelas, A., 2010. UF membrane fouling by mixtures of humic acids and sodium alginate: Fouling mechanisms and reversibility. Desalination 264, 220-227.

Kertész, S., László, Z., Horváth, Z., Hodúr, C., 2008. Analysis of nanofiltration parameters of removal of an anionic detergent. Desalination 221, 303-311. 
Kitis, M., Karanfil, T., Wigton, A., Kilduff, J., 2002. Probing reactivity of dissolved organic matter for disinfection by products formation using XAD-8 resin adsorption and UF fractionation. Water Research 36, 3834-3848.

Mosqueda-Jimenez, D., 2003. Impact of manufacturing conditions of Poly ethersulfone membranes on final characteristics and fouling reduction, $\mathrm{PhD}$ thesis, Department of Civil Engineering, University of Ottawa, Ottawa, ON.

Nagel, R., 1987. Seawater desalination with polyamide hollow fiber modules at DROP. Desalination 63, 225-246.

Peiris, R., Jaklewicz, M., 2013. Assessing the role of feed water constituents in irreversible membrane fouling of pilot-scale ultrafiltration drinking water treatment systems. Water Research 47, 3364 -3374.

Rodriguez, S., Amy, G., Schippers, J.C., Kennedy, M., 2015. The Modified Fouling Index Ultrafiltration constant flux for assessing particulate/colloidal fouling of RO systems. Desalination 365, 79-91.

Schippers, J., Verdouw, J., 1980. The modified fouling index: a method of determining the fouling characteristics of water. Desalination 32, 137-148. 
Taheri, A., Sim, L., Haur, C., Alkhondi, E., Fane, A., 2013. The fouling potential of colloidal silica and humic acid and their mixtures. Journal of Membrane Science 433, 112 120.

Villacorte, L., Ekowati, Y., Winters, H., Amy, G., Schippers, J., Kennedy, M., 2015. MF/UF rejection and fouling potential of algal organic matter from bloom-forming marine and freshwater algae. Desalination 367, 1-10.

Zazouli, M., Nasseri, S., Ulbricht, M., 2010. Fouling effects of humic and alginic acids in nanofiltration and influence of solution composition. Desalination 250, 688-692.

Zularisam, A., Ahmad, A., Sakinah, M., Ismail, A., Matsuura, T., 2011. Role of natural organic matter (NOM), colloidal particles, and solution chemistry on ultrafiltration performance. Separation and Purification Technology 78(2), 189-20. 


\title{
Chapter 4
}

\section{Investigation into the Temperature Effect on NOM Fouling and Cleaning in Submerged Polymeric Membrane Systems}

\author{
Mohammad T. Alresheedi ${ }^{*}$ and Onita D. Basu* \\ *Department of Civil and Environmental Engineering, Carleton University, 1125 Colonel \\ By Drive, Ottawa, ON, K1S 5B6, Canada. \\ Published: Journal of Desalination and Water Treatment
}

\begin{abstract}
Limited research on the influence of changing temperature conditions on reversible and irreversible NOM fouling has been conducted. Fouling and cleaning of a submerged polymeric ultrafiltration membrane with different NOM components (humic acids, proteins, polysaccharides, and their mixture) was examined at 5, 20, and $35^{\circ} \mathrm{C}$. Membrane fouling was evaluated using the MFI-UF and UMFI index methods, analysis of cake layer properties, and specific flux recovery. Results showed that fouling increased by $15-35 \%$ when water temperature decreased from $20^{\circ} \mathrm{C}$ to $5{ }^{\circ} \mathrm{C}$ whereas fouling decreased by 15 $25 \%$ when the temperature increased to $35^{\circ} \mathrm{C}$. The $\mathrm{UMFI}_{\mathrm{f}}$ fouling order was consistent across all temperature conditions with the NOM mixture and BSA fouling more severely than the alginate and humic acid. The UMFI and MFI-UF exhibited the same fouling order and can be used in complement to each other. BSA was found to be more sensitive to temperature changes and irreversibly foul more than humic acid and alginate. The ratio of irreversible to reversible fouling (UMFI $\mathrm{Uir}_{\text {ir }} / \mathrm{UMFI}_{\mathrm{hr}}$ ) increased by $15-25 \%$ from $35^{\circ} \mathrm{C}$ to 20
\end{abstract}


${ }^{\circ} \mathrm{C}$ and by $30-40 \%$ from $20{ }^{\circ} \mathrm{C}$ to $5{ }^{\circ} \mathrm{C}$ indicating the need for altered cleaning strategies at cold water conditions.

Keywords: Fouling indices; MFI-UF; NOM fouling; Temperature; UMFI

\subsection{Introduction}

Membrane processes are considered a reliable option for safe drinking water production. However, fouling by natural organic matter (NOM) (such as humic, protein, and polysaccharide like substances) creates significant challenges for membrane processes to maintain good performance during operation. Fouling can decrease membrane efficiency, increase operating costs, and ultimately decrease the lifetime of the membrane.

Water temperature is a key design parameter that influences membrane operation in terms of fouling and cleaning. However, studies that have examined temperature associated impacts with membranes are more commonly done with high pressure systems (nanofiltration (NF) and reverse osmosis (RO)) at temperatures greater than $20^{\circ} \mathrm{C}$. These studies tend to focus more on solution viscosity and solute diffusivity challenges with operation but not changes in fouling behavior (Zhao and Zhou, 2011; Jin et al., 2009). Other studies have investigated temperature impacts with wastewater MBR systems (Ma et al., 2013; Gao et al., 2012), whereas drinking water low pressure membrane studies with a focus on lower temperatures are very limited (Steinhauer et al., 2015). In particular, the effect of low water temperatures (e.g. $5^{\circ} \mathrm{C}$ ) have not been investigated in depth. Drinking water systems have in general neglected the interplay of water temperature and fouling on low pressure membrane systems. Although one study with low pressure membrane systems 
found that current standards for membrane integrity testing overestimated safety design values by not taking into account temperatures below $5{ }^{\circ} \mathrm{C}$ (Farahbakhsh et al., 2006) highlighting the need for this type of research. Very little is known on the effect of water temperature on NOM fouling and cleaning of low pressure polymeric membranes which are critical for their design and operation for drinking water production.

Researchers have developed several methods to quantify fouling for membrane systems. Blocking laws quantify fouling location such as within the pores, pore blocking, and cake layer (Hermia et al., 1982). Fouling indices, which are considered simple and short filtration tests, are commonly used to predict the fouling potential of the membrane feed. The silt density index (SDI) and modified fouling index (MFI), using a $0.45 \mu \mathrm{m}$ pore size membranes, have been commonly used as fouling predictors for RO membranes (ASTM, 2014; Schippers and Verdouw, 1980). Boerlage et al. (2002) developed the MFI-UF, making use of ultrafiltration (UF) membranes to count for particles $<0.45 \mu \mathrm{m}$. Feed water having an MFI-UF $<3000 \mathrm{~s} / \mathrm{L}^{2}$ is equivalent to $\mathrm{SDI}<3 \% / \mathrm{min}$, which is considered acceptable for membrane feed (Boerlage et al., 2003; Boerlage et al., 2002). Few studies applied the MFI-UF for assessing biofouling with RO systems (Jeong and Vigneswaran, 2015; Villacorte et al., 2015). Other studies investigated the influence of inorganic silica and calcium colloids on the MFI-UF fouling index with RO systems (Taheri et al., 2013). However, limited to no research exists that evaluates the use of the MFI-UF to predict NOM fouling for low pressure membrane systems under changes in water temperature condition. 
The unified membrane fouling index (UMFI) model (Huang et al., 2008) has been applied to quantify fouling based on hydraulic and chemical reversibility and irreversibility components. Although different fouling types, i.e., reversible versus irreversible, are well defined in the literature, inconsistent bench scale results have been reported regarding membrane fouling by NOM, possibly due to different testing conditions, with many deviations from full-scale practice (Hashino et al., 2011; Katsoufidou et al., 2010; Xiao et al., 2009). These studies were often conducted for short durations at conditions that do not include hydraulic backwash cycles in the testing and use of different membrane materials. Moreover, correlations between MFI-UF and UMFI for assessing NOM fouling in polymeric membrane systems under changes in water temperature condition needs to be clarified. In this research, filtration performance, NOM fouling and chemical cleaning of a submerged polymeric UF membranes across a range of feed water temperatures $(5,20$, and $35^{\circ} \mathrm{C}$ ) are examined. The UMFI method is utilized to assess for respective reversible and irreversible fouling; while the MFI-UF is used to assess its effectiveness for predicting NOM fouling in general and under changing temperature conditions. The temperature impact on chemical cleaning is investigated with respect to membrane resistance and permeability recoveries with the various NOM solutions.

\subsection{Materials and Methods}

\subsubsection{Feed solutions}

Four different synthetic feed water solutions were used in this study to cover the range of hydrophobic and hydrophilic NOM types typically found in surface water sources (Hashino et al., 2011; Katsoufidou et al., 2010; Jermann et al., 2008). Humic acids (2.5 mgC/L); a 
protein (bovine serum albumin, $\mathrm{BSA}, 2.5 \mathrm{mgC} / \mathrm{L}$ ); a polysaccharide (sodium alginate, 2.5 $\mathrm{mgC} / \mathrm{L})$; and a mixture of the three NOM models $(0.83 \mathrm{mgC} / \mathrm{L} /$ each NOM model, total of $2.5 \mathrm{mgC} / \mathrm{L}$ ), were used as model NOM foulants. All model substances were purchased from Sigma Aldrich. A moderate hardness and alkalinity of $75 \mathrm{mg} / \mathrm{L}$ calcium carbonate $\left(\mathrm{CaCO}_{3}\right)$ and a low level of turbidity (5 NTU) as kaolin clay particles were included in the synthetic water matric to represent the more complex conditions of a surface water source (Jermann et al., 2008).

Feed solutions were prepared using DI water and were mixed using a magnetic stirrer oneday prior any experiment to ensure that materials were dissolved completely. Feed water was continuously mixed using a VWR dual speed mixer to ensure homogeneous water conditions throughout the experiment. The feed tank was insulated to maintain constant temperature throughout the testing period. An immersion heater (Cole Parmer) and a compact chiller (LM series, Polyscience) were used to adjust the water temperature as required. Temperature and $\mathrm{pH}$ were monitored continuously using $\mathrm{HACH}$ (cat.no. 5825800) HQd Field Case equipment. The $\mathrm{pH}$ of the feed was adjusted as needed to 7.5 with $\mathrm{NaOH}$. The molecular weight distribution and zeta potential of the feed solutions, which are comprised of the respective NOM components, kaolin clay, and calcium, were measured using a UF fractionation method described by Kitis et al. (2002) (shown in Table 4.1). A Malvern Nano-sizer was used to determine the zeta potential of feed water solutions. Triplicate measurements were performed for each sample. 
Table 4.1- Molecular weight fractionation and zeta potential of feed solutions

\begin{tabular}{ccccc}
\hline \multicolumn{5}{c}{ Model Feed Solutions } \\
\hline Molecular Weight & Humic acid & BSA & Alginate & Mixture \\
\hline$>100 \mathrm{kDa}$ & $38 \%$ & $51 \%$ & $36 \%$ & $55 \%$ \\
$30 \mathrm{kDa}-100 \mathrm{kDa}$ & $42 \%$ & $44 \%$ & $48 \%$ & $42 \%$ \\
$10 \mathrm{kDa}-30 \mathrm{kDa}$ & $10 \%$ & $2 \%$ & $6 \%$ & $2 \%$ \\
$5 \mathrm{kDa}-10 \mathrm{kDa}$ & $6 \%$ & $1 \%$ & $3 \%$ & $1 \%$ \\
$1 \mathrm{kDa}-5 \mathrm{kDa}$ & $5 \%$ & $2 \%$ & $4 \%$ & $1 \%$ \\
$<1 \mathrm{kDa}$ & $2 \%$ & $1 \%$ & $2 \%$ & $1 \%$ \\
\hline Zeta Potential $(\zeta)$ & $-22 \pm 5$ & $-18 \pm 4$ & $-20 \pm 5$ & $-16 \pm 7$ \\
\hline
\end{tabular}

\subsubsection{Experimental setup and approach}

\subsubsection{Submerged polymeric membrane setup}

Two submerged membrane systems were evaluated in parallel as shown in Figure 4.1. Zenon ZW-1 hollow fiber (UF) membranes made of polyvinylidene fluoride (PVDF), with a nominal pore size of $0.04 \mu \mathrm{m}$ (surface area of $0.047 \mathrm{~m}^{2}$ ) were used and operated under a vacuum pressure between 0 and -8.7 psi ( 0 and 0.6 bar). Filtration experiments were performed for 24 hrs under the operational conditions presented in Table 4.2. These operational conditions were applied based on previous work by De Souza and Basu (2013) and Alresheedi and Basu (2014). The flow rate and direction of permeate (i.e. forward and backward) were controlled using WinLN software. Pressure readings were recorded every 10 seconds using Operational Flux 2.0 program. 
Table 4.2- Operational conditions of filtration experiments

\begin{tabular}{cc}
\hline Parameter & \\
\hline Flux & $38 \mathrm{LMH}$ \\
Filtration Cycle & 15 minutes \\
Filtration Mode & Dead-end \\
Backwash Duration & 20 seconds \\
Air Scour & $5 \mathrm{~L} /$ min $(\mathrm{LPM})$ \\
Total Filtration Time & 24 hours \\
\hline
\end{tabular}

To assess the potential seasonal impacts of water temperature on membrane fouling and cleaning, feed water temperature was varied between 5,20 , and $35{ }^{\circ} \mathrm{C}$. Equation 4.1 (Crittenden et al., 2005) was used to correct flux measured at different temperature to standard temperature of $20^{\circ} \mathrm{C}$. This is important as the flux greatly depends on water temperature.

$\mathrm{J}_{20}{ }^{\circ} \mathrm{C}=\mathrm{J}_{\mathrm{m}}(1.03)^{\mathrm{T}} 20^{\circ} \mathrm{C}^{-\mathrm{T}} \mathrm{m} \quad$ Equation (4.1)

$\mathrm{J}_{20}{ }^{\circ} \mathrm{C}$ and $\mathrm{T}_{20}{ }^{\circ} \mathrm{C}$ are the flux and temperature at $20{ }^{\circ} \mathrm{C}$, respectively. $\mathrm{J}_{\mathrm{m}}$ and $\mathrm{T}_{\mathrm{m}}$ are the measured flux and temperature, respectively.

Membranes were cleaned in place (CIP) between each experiment following manufacture specified recommendations. Cleaning of the membrane units was done in two steps: soaking in a $250 \mathrm{ppm}$ of sodium hypochlorite $(\mathrm{NaOCl})$ solution for 4 hours followed by soaking in citric acid solution for another 4 hours. Chemical cleaning was conducted at room temperature. DI water was then filtered through the membranes at permeate flux of $38 \mathrm{LMH}$ and the membrane permeate flow was measured to ensure that the membranes were able to achieve the required flux before being placed back in operation. 


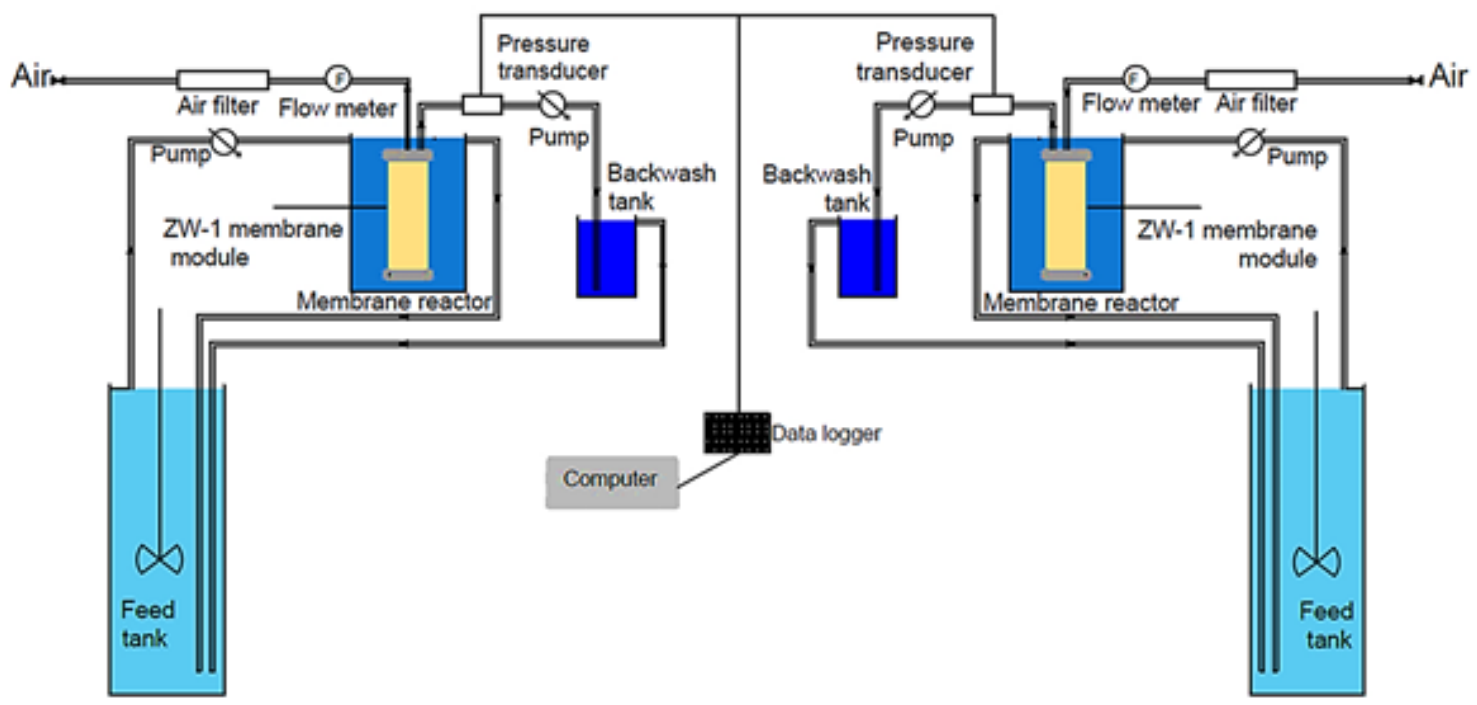

Figure 4.1- A schematic representation of the bench scale polymeric membrane setups

4.2.2.2 Fouling resistances using the unified membrane fouling index (UMFI) method

To assess polymeric membrane's fouling at different water temperature conditions, the UMFI model, Equation 4.2 (Huang et al., 2008) was applied to quantify each contributing fouling resistance.

$\frac{1}{\mathrm{~J}_{\mathrm{s}}{ }^{\prime}}=1+(\mathrm{UMFI}) \mathrm{x} \mathrm{Vs}$

Equation (4.2)

Where $\mathrm{J}_{\mathrm{S}}$ is the normalized specific flux (unitless), UMFI is an estimate of the extent of fouling $\left(\mathrm{m}^{-1}\right)$, and $\mathrm{V}_{\mathrm{S}}\left(\mathrm{m}^{3} / \mathrm{m}^{2}\right)$ is the specific permeate volume. UMFI can be calculated using Equation 4.3.

$\mathrm{UMFI}_{\mathrm{f}}=\mathrm{UMFI}_{\mathrm{hr}}+\mathrm{UMFI}_{\mathrm{hir}}=\mathrm{UMFI}_{\mathrm{hr}}+\mathrm{UMFI}_{\mathrm{cr}}+\mathrm{UMFI}_{\mathrm{cir}} \quad$ Equation (4.3)

Where $\mathrm{UMFI}_{\mathrm{f}}$ is the total fouling resistance index; $\mathrm{UMFI}_{\mathrm{hr}}$ is hydraulically reversible fouling resistance index (i.e. removed by backwash); $\mathrm{UMFI}_{\text {hir }}$ is hydraulically irreversible 
fouling resistance index (i.e. remained after backwash). $\mathrm{UMFI}_{\text {hir }}$ can be divided into $\mathrm{UMFI}_{\mathrm{cr}}$ and $\mathrm{UMFI}_{\mathrm{cir}}$. UMFI $\mathrm{cr}$ is chemically reversible fouling resistance index (i.e., removable by chemical cleaning); and $\mathrm{UMFI}_{\text {cir }}$ is chemically irreversible fouling resistance index (i.e., remained after chemical cleaning).

Figure 4.2 illustrates the UMFI method used to calculate different fouling resistances. $\mathrm{UMFI}_{\mathrm{f}}$ was calculated by using filtration data from the start to end of filtration. $\mathrm{UMFI}_{\text {hir }}$ represent the slope of the line after the start of each filtration cycle. $\mathrm{UMFI}_{\mathrm{hr}}$ is the difference between $\mathrm{UMFI}_{\mathrm{f}}$ and $\mathrm{UMFI}_{\text {hir. }} \mathrm{UMFI}_{\text {cir }}$ was calculated by collecting data after the chemical cleaning step. $U M F I_{c r}$ is the difference between $U M F I_{\text {hir }}$ and $U M F I_{c i r}$.

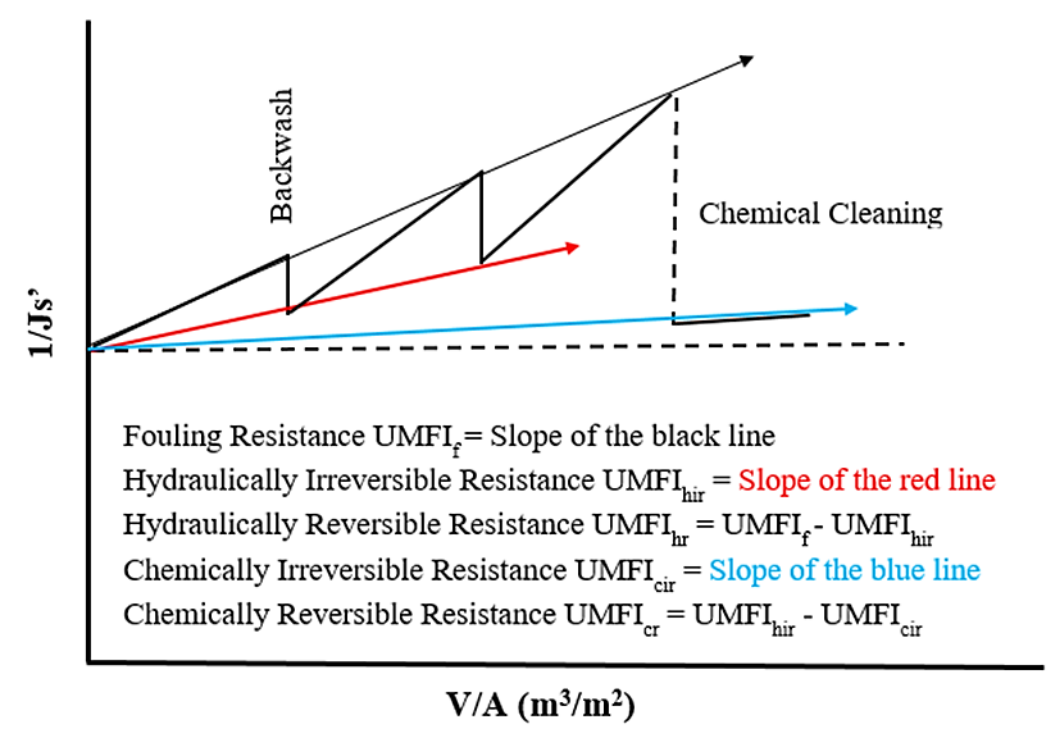

Figure 4.2 - Estimation of fouling resistances using the UMFI method (adapted from Alresheedi et al., 2017) 


\subsubsection{Specific cake resistance}

According to Darcy's law, the flux through a clean membrane can be written as:

$\mathrm{J}=\frac{\Delta \mathrm{P}_{0}}{\mu \mathrm{R}_{\mathrm{m}}}$

$\mathrm{J}$ is the membrane flux $\left(\mathrm{m}^{3} / \mathrm{m}^{2} . \mathrm{s}\right) ; \Delta \mathrm{P}_{0}$ is initial transmembrane pressure $(\mathrm{kPa}) ; \mathrm{R}_{\mathrm{m}}$ is the intrinsic membrane resistance $\left(\mathrm{m}^{-1}\right)$; and $\mu$ is the viscosity of the fluid ( $\left.\mathrm{kPa} \mathrm{s}\right)$.

For fouled membrane, if all fouling is assumed to be a result of cake filtration, then Equation 4.4 can be rewritten as:

$J=\frac{\Delta P}{\mu\left(R_{C}+R_{m}\right)}$

Equation (4.5)

$\Delta \mathrm{P}$ is final transmembrane pressure $(\mathrm{kPa}) ; \mathrm{Rc}$ is the cake layer resistance $\left(\mathrm{m}^{-1}\right)$.

The Rc can be expressed in terms of the specific cake resistance $\alpha_{C}(\mathrm{~m} / \mathrm{kg})$, the bulk concentration, $\mathrm{C}_{\mathrm{b}}\left(\mathrm{kg} / \mathrm{m}^{3}\right)$, and the volume of filtered water per unit membrane area $\left(\mathrm{V}_{\mathrm{s}}\right)$, as follows:

$\mathrm{R}_{\mathrm{C}}=\alpha_{\mathrm{c}} \mathrm{C}_{\mathrm{b}} \mathrm{V}_{\mathrm{S}}$

Equation (4.6)

Equations 4.4-4.6 can be combined to express the increase in transmembrane pressure at constant flux during cake filtration as shown in Equation 4.7 (Chellam and Wendong, 2006).

$\Delta \mathrm{P}=(\mathrm{J} \mu)\left(\alpha_{\mathrm{c}} \mathrm{C}_{\mathrm{b}} \mathrm{V}_{\mathrm{S}}+\mathrm{R}_{\mathrm{m}}\right)=\Delta \mathrm{P}_{0}+\left(\mathrm{J} \mu \mathrm{C}_{\mathrm{b}} \mathrm{V}_{\mathrm{S}}\right) \alpha_{\mathrm{c}}$ Equation (4.7)

The specific cake resistance $\alpha_{c}$ often increases according to a power law (Foley et al., 2006) as shown in Equation 4.8: 
$\alpha_{\mathrm{C}}=\alpha_{0} \Delta \mathrm{Pn}$

Equation (4.8)

in which $\alpha_{0}$ is a constant related primarily to the size and shape of the particles forming the cake, $\Delta \mathrm{P}$ is final pressure, and $\mathrm{n}$ is the cake compressibility index, which ranges from zero (incompressible cake) to 1 or greater (for a highly compressible cake).

Scanning electron microscopy (SEM) analysis was conducted using $0.45 \mu \mathrm{m}$ membrane filters fouled for 15 minutes at different feed water temperatures. SEM images were taken at $10.00 \mathrm{kx}$ magnification using the Tescan Vega-II XMU equipment for more insights into the effect of temperature on the fouling layer of different NOM fractions.

\subsubsection{MFI-UF fouling index}

Fouling potential of feed water solutions from the submerged membrane experiments (i.e. humic acids, BSA, alginate, and NOM mixture), was assessed using the MFI-UF method (Boerlage et al., 2002). Fouling indices are used to measure and predict the fouling potential of membrane feedwater, diagnose fouling at the design stage, and/or monitor pretreatment performance during membrane operation. The MFI-UF setup is described in detail by Boerlage et al. (2002); Alresheedi et al. (2018). The characteristics of the MFIUF membrane are presented in Table 4.3. 
Table 4.3 - MFI-UF membrane characteristics

\begin{tabular}{cc}
\hline & MFI-UF Membrane \\
\hline Membrane Type & UF - 13 kDa \\
Membrane Materials & PAN hollow fiber (Pall Corp.) \\
Configuration & Inside-out \\
\hline Fiber ID & $0.8 \mathrm{~mm}$ \\
Fiber OD & $1.4 \mathrm{~mm}$ \\
Number of Fiber & 400 \\
\hline Membrane Area & $0.2 \mathrm{~m}^{2}$ \\
Module Length & $347 \mathrm{~mm}$ \\
Module Diameter & $42 \mathrm{~mm}$ \\
\hline
\end{tabular}

MFI-UF testing was determined according to method described by Boerlage et al. (2002).

Feed water was filtered through the hollow fiber UF membrane under dead-end mode and pressure of 2 bars. Permeate was collected in a tank set on the electronic balance to acquire permeate volume (V) and filtration time ( $\mathrm{t}$ ) data from the balance. The MFI-UF exp was then calculated using Equation 4.9. $\mathrm{d}(\mathrm{t} / \mathrm{V}) / \mathrm{dV}$ is the slope of two data points in the linear region of $\mathrm{t} / \mathrm{V}$ versus $\mathrm{V}$ graph (described in detail by Boerlage et al. (2002).

MFI-UF exp $=\frac{d\left(\frac{t}{v}\right)}{d V} \quad$ Equation (4.9)

The MFI-UF test was performed under the same water temperature conditions as the submerged polymeric membranes experiments (i.e. 5 , 20, and $35^{\circ} \mathrm{C}$ ).

\subsection{Results and Discussion}

\subsubsection{Effect of water temperature condition on NOM fouling}

Figures 4.3A-4.3D show the inverse of the normalized specific flux $\left(1 / \mathrm{J}_{\mathrm{s}}{ }^{\prime}\right)$ versus filtered water volume per membrane area (V/A). Water temperature was found to impact NOM 
fouling as follows: an increase in water temperature resulted in lower fouling rate for all NOM solutions. Overall, as temperature decreased from $20{ }^{\circ} \mathrm{C}$ to $5{ }^{\circ} \mathrm{C}$, membrane fouling increased by $15-35 \%$ while fouling decreased by $15-25 \%$ when the temperature increased from $20{ }^{\circ} \mathrm{C}$ to $35{ }^{\circ} \mathrm{C}$. The changes in NOM fouling behavior with temperature could be attributed to the changes in fouling layer resistance and NOM retention (Kertész et al., 2008). Thus, changes in water temperature condition could be crucial factor for membrane systems to meet consistent performance targets.

Figure 4.3 also shows that different NOM fractions responded differently to temperature changes. BSA filtration is more sensitive to temperature changes in which fouling was observed to increase by $35 \%$ with decreasing temperature from $20^{\circ} \mathrm{C}$ to $5^{\circ} \mathrm{C}$ compared to $15 \%$ and $20 \%$ for humic acid and alginate, respectively. In addition, fouling of the NOM mixture is approximately similar to BSA alone, indicating a higher effect from protein substances on fouling. The differences between fouling among the types of NOM could be partially attributed to the molecular weight distribution and zeta potential (as shown in Table 4.1). For humic acid and sodium alginate feedwater solutions, there were larger components with molecular weight in the range of 30-100 kDa which was smaller than the pore size of the membrane $(0.04 \mu \mathrm{m})$ and may therefore adsorb on the pore surface and caused internal fouling or passed through the membrane to permeate side. On the other hand, BSA and NOM mixture feedwater solutions had a larger fraction of components with molecular weight $>100 \mathrm{kDa}$ which may have caused pore blocking during the early stages of filtration and/or adsorbed on the pore surface leading to higher fouling compared to humic acid and sodium alginate. 
Moreover, the zeta potential values of BSA and NOM mixture feed water (-18 $\mathrm{mV}$ and $16 \mathrm{mV}$ respectively) were lower than that for humic acid and alginate feed water $(-22 \mathrm{mV}$ and $-20 \mathrm{mV}$ respectively) and would have a higher tendency to adsorb on the membrane surface or pores. Similar results were reported by Contreras et al. (2009) study in which BSA has a lower zeta potential $(-20.7 \mathrm{mV})$ compared to sodium alginate $(-45.0 \mathrm{mV})$ and humic acid (-37.9) leading to lower electrostatic repulsion and higher hydrophobic interaction between BSA and thin-film composite NF membrane. Interestingly, sodium alginate (hydrophilic) resulted in higher fouling compared to humic acid (hydrophobic) indicating a strong influence from polysaccharides on membrane fouling compared to humic substances. These results are consistent with those reported by Hashino et al. (2011) in which the sodium alginate solution showed more severe flux decline than humic acid with MF membranes.
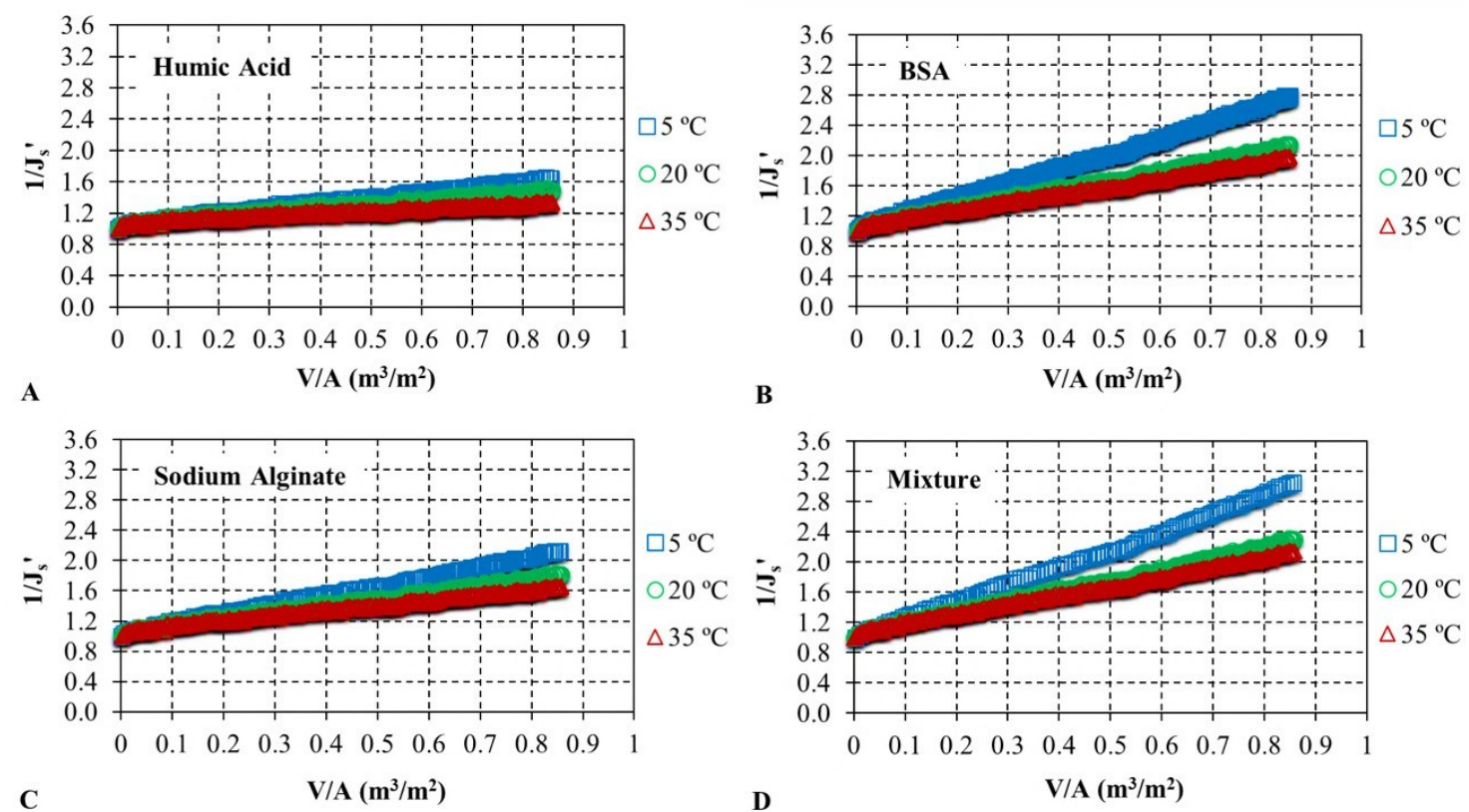

B

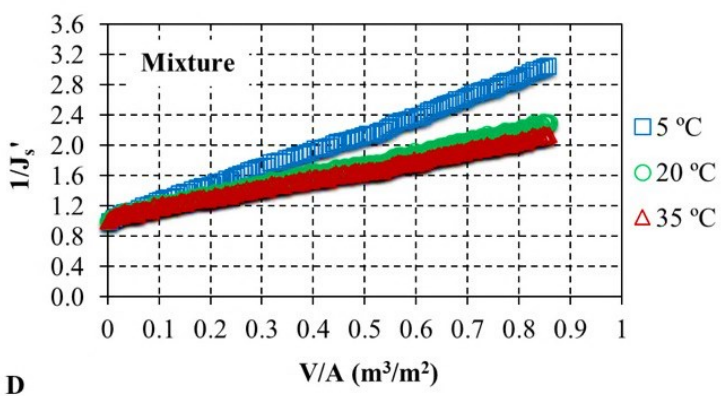

Figure 4.3 - NOM Fouling graphs (A) Humic Acid; (B) BSA; (C) Sodium Alginate; (D) Mixture. Js': normalized specific flux; V/A: filtered water volume per membrane area 
Additionally, the specific cake resistance $\left(\alpha_{c}\right)$ and compressibility index (n) were calculated from the experimentally determined values of the filtration pressure, bulk concentration, and specific filtered water volume, considering cake filtration is the dominant fouling mechanism (Alresheedi et al., 2018), as described in Equations 4.4-4.8. Figures 4.4A shows that $\alpha_{c}$ value of all NOM models decreases as feed water temperature increases from 5 to $35{ }^{\circ} \mathrm{C}$, therefore, temperature influences the NOM cake structure. Jin et al. (2009) found that humic acid colloids decreased in size with increasing temperature of RO feed. As a result, smaller particles produced more porous deposits and influenced fouling. In this study, the decrease in $\alpha_{c}$ with increasing temperature could be attributed to the decrease in NOM size which may have changed the cake layers into more porous and open structures. Thus, lower fouling was obtained at higher temperature. Furthermore, Figures 4.4A and 4.4B show that BSA and NOM mixture cake resistances are significantly affected by temperature; changes in cake resistance with temperature indicate more compressible cake ( $\mathrm{n}=0.85$ and 0.89 for BSA and NOM mixture, respectively). This is reflected in Figure 4.3, where the fouling trend for BSA and NOM mixture changes significantly, particularly between $5{ }^{\circ} \mathrm{C}$ and $20^{\circ} \mathrm{C}$. The compressibility indices for humic acid and sodium alginate are quite similar ( 0.57 and 0.59 , respectively) which differ from those of Sioutopoulos et al. (2010) who found that humic acid deposits are more compressible $(n=0.70)$ compared with alginate deposits $(n=0.40)$ with UF membranes. The difference in the actual numerical value of $n$ may be caused by the different membrane material and/or the high concentration of total dissolved solids in the solutions used by Sioutopoulos et al. (2010). 

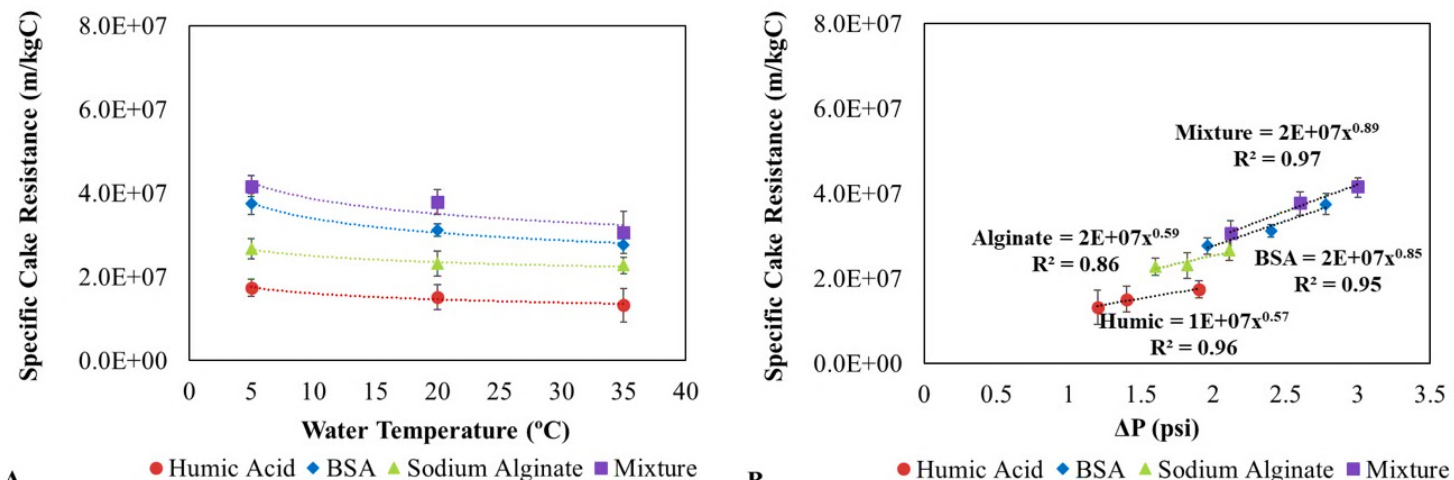

A

- Humic Acid • BSA $\triangle$ Sodium Alginate $=$ Mixture

B

- Humic Acid $\bullet$ BSA $\triangle$ Sodium Alginate $\square$ Mixture

Figure 4.4 - (A) Changes in specific cake resistance with temperature; (B) estimated compressibility index values

To visualize the effect of temperature on NOM fouling layer properties, Figure 4.5 shows SEM images of humic acid, BSA, and sodium alginate fouling layers. The SEM analysis were conducted using $0.45 \mu \mathrm{m}$ membrane filters fouled for 15 minutes at constant pressure of 1 bar and different feed water temperatures. From Figure 4.5, it can be clearly seen that the fouling layers differ between different NOM components. At $20^{\circ} \mathrm{C}$, humic acid filled the pores partially and developed a layer of relatively open structure whereas protein and sodium alginate developed a rough gel layer and appeared to fill more pores compared to humic acid. This is reflected in Figure 4.3, where the fouling for BSA and alginate was higher than humic acid. Figure 4.5 also shows a decrease in water temperature from $20^{\circ} \mathrm{C}$ to $5{ }^{\circ} \mathrm{C}$ resulted in more compact layer for all NOM components. More specifically, at 5 ${ }^{\circ} \mathrm{C}$, BSA completely blocked membrane pores and developed a fouling layer of high resistance. This can be supported by the findings from Figure 4.4 in which BSA has the highest specific fouling resistance and compressibility index values compared to alginate and humic acid. 


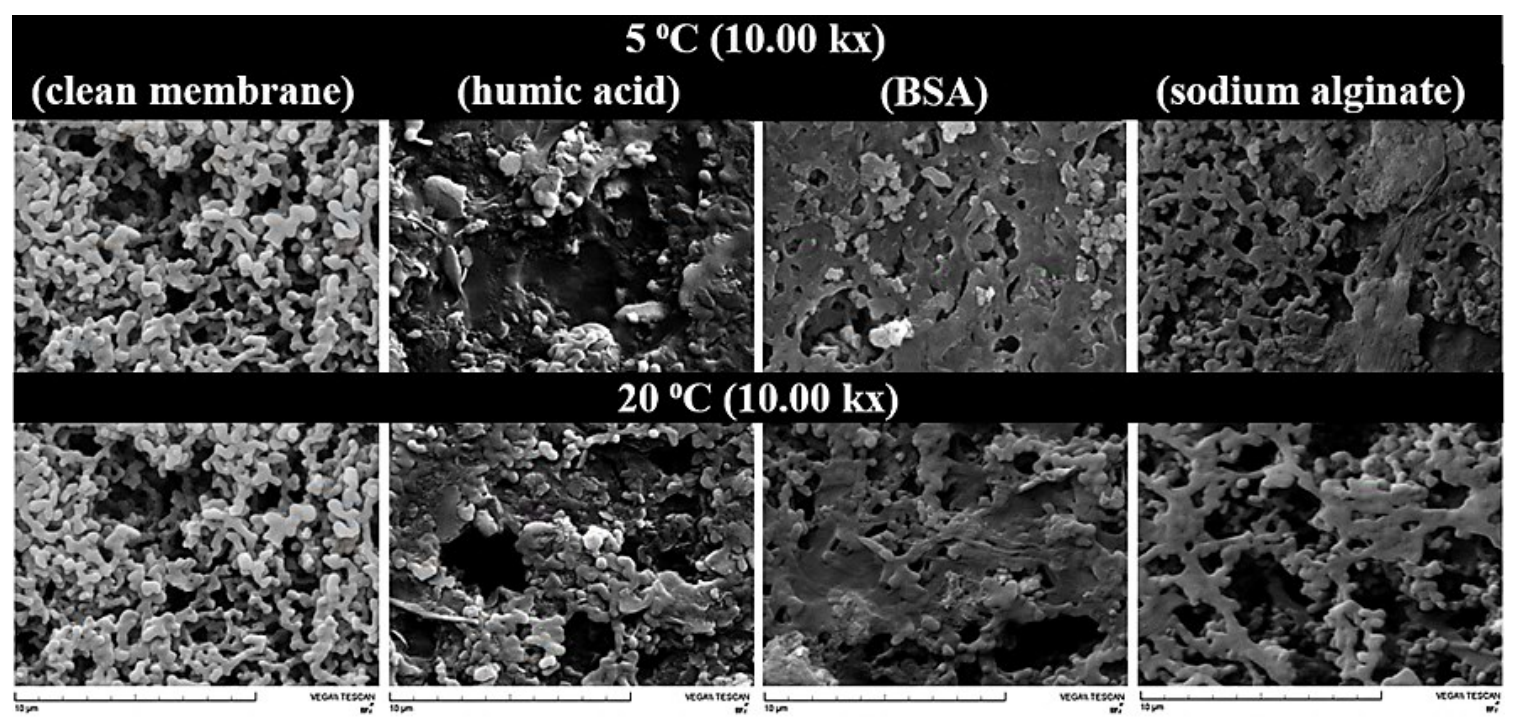

Figure 4.5 - SEM images of NOM fouling layers at $5{ }^{\circ} \mathrm{C}$ and $20{ }^{\circ} \mathrm{C}(\mathrm{P}=1$ bar $)$ using $0.45 \mu \mathrm{m}$ membrane filters.

\subsubsection{Fouling indices assessment at different water temperature conditions}

\subsubsection{Reversible and irreversible fouling indices}

To quantify membrane fouling during membrane filtration, fouling resistances using the unified membrane fouling index method (UMFI) were estimated. The lower the $\mathrm{UMFI}_{\mathrm{hir}} / \mathrm{UMFI}_{\mathrm{hr}}$ ratio the more effective the hydraulic backwashes, due to greater cake formation. While higher the $\mathrm{UMFI}_{h i r} / \mathrm{UMFI}_{\mathrm{hr}}$ ratio the greater the $\mathrm{NOM}$ adsorption and pore blockage and thus a greater need for chemical cleans as hydraulic backwashes are less effective. The hydraulically irreversible fouling index (UMFI $\left.{ }_{\text {hir }}\right)$ can be categorized into the chemically reversible fouling $\left(\mathrm{UMFI}_{\mathrm{cr}}\right)$ or the chemically irreversible fouling $\left(\mathrm{UMFI}_{\mathrm{cir}}\right)$. $\mathrm{UMFI}_{\mathrm{cr}} / \mathrm{UMFI}_{\text {hir }}$ quantifies the irreversible fouling ratio removed by a chemical clean while $\mathrm{UMFI}_{\mathrm{cir}} / \mathrm{UMFI}_{\text {hir }}$ quantifies the fouling ratio that remains after a chemical clean. Minimizing chemical cleans is preferred to help control membrane aging which decreases the hydraulic resistance and hydrophobicity of membrane material (Puspitasari et al., 2010). Moreover, controlling the degree of chemical cleaning helps minimize chemical 
waste production and maintain membrane productivity. Figure 4.6A-4.6C shows the UMFI values determined at different tested water temperature conditions. The $\mathrm{UMFI}_{\mathrm{f}}$ fouling index order was consistent at different temperature which was the highest for the NOM mixture followed by BSA, alginate, and lastly humic acid. The similar $\mathrm{UMFI}_{\mathrm{f}}$ values for the NOM mixture and the BSA solution indicate that protein-based NOM significantly contributes to fouling in membrane systems. Hashino et al. (2011) similarly reported a significant flux decline of cellulose acetate butyrate membrane caused by BSA adsorption compared to alginate. Moreover, BSA fouling, refer to Figure 4.6, is more sensitive to changes in water temperature condition in which $\mathrm{BSA} \mathrm{UMFI}_{\mathrm{f}}$ increased by $35 \%$ with decreasing temperature from $20{ }^{\circ} \mathrm{C}$ to $5^{\circ} \mathrm{C}$ compared to $15 \%$ and $20 \%$ for humic acid and alginate, respectively. 


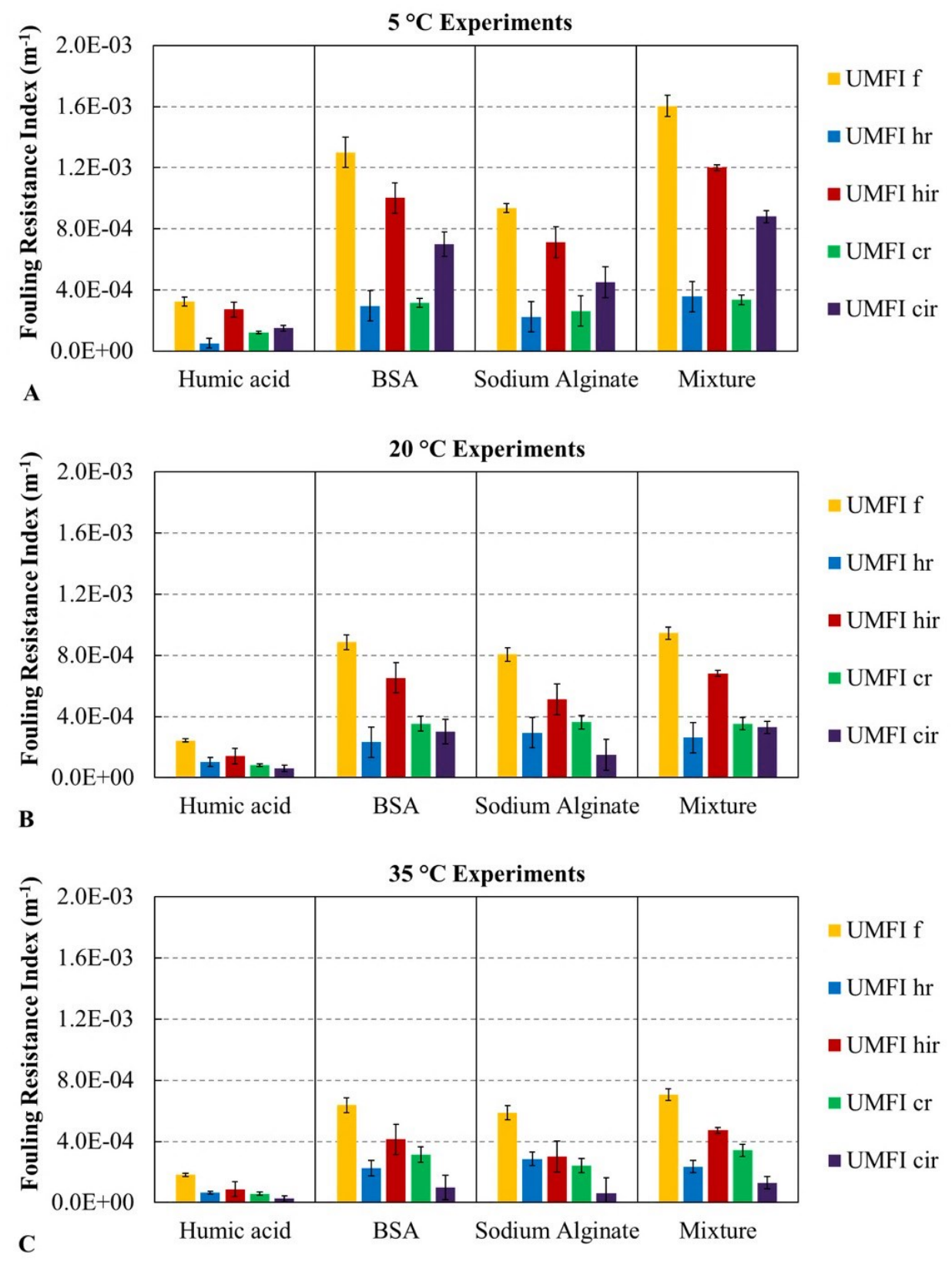

Figure 4.6 - Estimated UMFI values at different temperature conditions.

*Note: UMFIf: unified total fouling index; $\mathrm{UMFI}_{\mathrm{hr}}$ : unified hydraulically reversible fouling index; $\mathrm{UMFI}_{\text {hir }}$ : unified hydraulically irreversible fouling index; $\mathrm{UMFI}_{\mathrm{cr}}$ : unified chemically reversible fouling index; $\mathrm{UMFI}_{\mathrm{cir}}$ : unified chemically irreversible fouling index.

To quantify the effect of water temperature on NOM fouling behaviors, Figure 4.7 presents the changes in irreversible to reversible fouling index ratios $\left(\mathrm{UMFI}_{\text {hir }} / \mathrm{UMFI}_{\mathrm{hr}}\right)$ under all 
tested water temperature conditions. The $\mathrm{UMFI}_{\text {hir }} / \mathrm{UMFI}_{\mathrm{hr}}$ ratios of all $\mathrm{NOM}$ models was highest during the $5{ }^{\circ} \mathrm{C}$ operation, $(1.8$ to 3.3$)$, compared to $20{ }^{\circ} \mathrm{C},(0.95$ to 1.9$)$, and $35^{\circ} \mathrm{C}$, (0.7 to 1.3$)$. The increase in the $\mathrm{UMFI}_{\mathrm{hir}} / \mathrm{UMFI}_{\mathrm{hr}}$ at lower temperature indicates lower hydraulic backwash efficiency, thus, higher NOM irreversibility. This is consistent with the increase in specific cake resistance observed in Figure 4.4A. The $\mathrm{UMFI}_{\text {hir }} / \mathrm{UMFI}_{\text {hr }}$ ratios decreased by about $30-40 \%$ as feed water temperature increased from $5{ }^{\circ} \mathrm{C}$ to $20{ }^{\circ} \mathrm{C}$ and by about $15-25 \%$ as the temperature increased from $20{ }^{\circ} \mathrm{C}$ to $35^{\circ} \mathrm{C}$. Therefore, hydraulic backwashing at warm water temperature (i.e. 20 and $35^{\circ} \mathrm{C}$ ) was more effective in removing the hydraulically irreversible fouling of NOM compared to cold water temperature, $5{ }^{\circ} \mathrm{C}$. Irreversibility of the foulant layer is a major concern during membrane filtration, as it influences hydraulic or chemical cleaning requirements. Although hydraulic backwashing was efficient in mitigating a large percentage of NOM fouling at warm water temperature condition, the significant amount of irreversible fouling due to stronger adsorption of foulants actually poses a potential limitation to longer membrane operation.

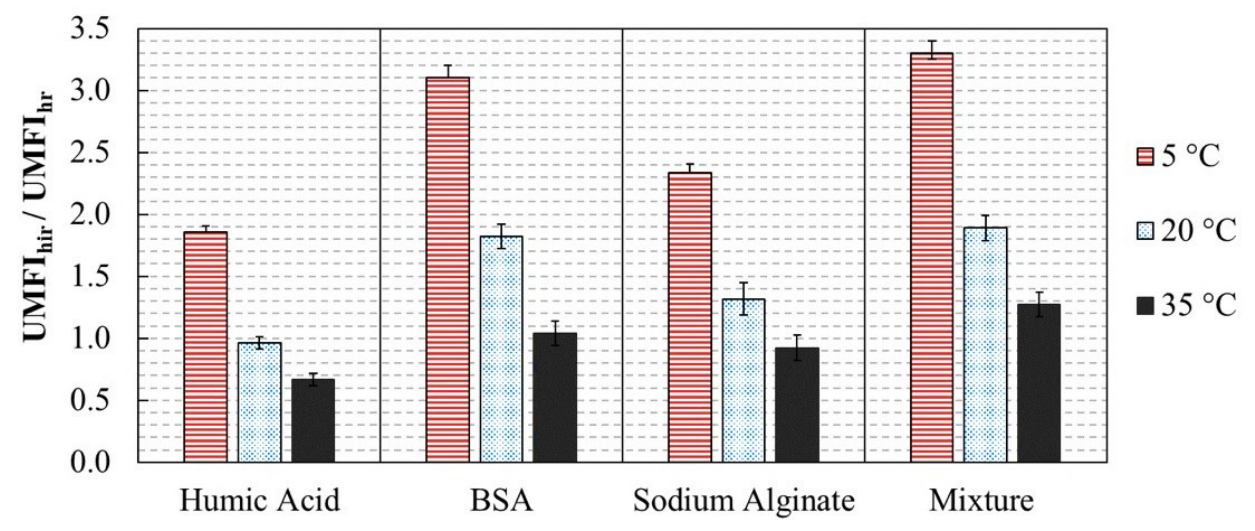

Figure 4.7 - Hydraulically irreversible to reversible fouling index ratios at different water temperature conditions*

*Note: $\mathrm{UMFI}_{\text {hir }} / \mathrm{UMFI}_{\mathrm{hr}}$ ratio of 1 indicates $50 \%$ irreversible and $50 \%$ reversible fouling. Ratio $>1$ indicates higher irreversible fouling. 


\subsubsection{Correlation of UMFI and MFI-UF indices}

The MFI-UF method is used to assess the fouling tendency of a membrane feed whereas the UMFI provides information on the current fouling a membrane is subjected to. Thus, the MFI-UF is used to predict fouling while the UMFI is used to quantify fouling in an operating system. Feed water having an MFI-UF $<3000 \mathrm{~s} / \mathrm{L}^{2}$ (equivalent to an $\mathrm{SDI}<3$ $\% / \mathrm{min}$ ), is considered acceptable for membrane feed (Boerlage et al., 2002). However, the actual capacity of the MFI-UF to be used effectively as a prediction tool with NOM has yet to be evaluated in general and under changing water temperature conditions. Table 4.4 presents fouling index values predicted by the MFI-UF method. The results in Table 4.4 show an increased fouling potential across all NOM sources as the water temperature drops which is in agreement with the increased fouling tendency observed in Figure 4.7 using the UMFI method. The NOM fouling potential predicted by the MFI-UF was in the order of mixture $>$ BSA $>$ sodium alginate $>$ humic acid which matches the fouling trend observed in the submerged polymeric UF system with the UMFI method.

Table 4.4 -MFI-UF index prediction of NOM fouling at different temperatures

\begin{tabular}{ccccr}
\hline & Humic Acid & BSA & Sodium Alginate & Mixture \\
\hline \multicolumn{4}{c}{ MFI-UF (s/L $\left.\mathbf{L}^{2}\right)$} \\
\hline $5{ }^{\circ} \mathrm{C}$ & $5559 \pm 294$ & $9880 \pm 332$ & $6948 \pm 186$ & $10122 \pm 491$ \\
$20^{\circ} \mathrm{C}$ & $3487 \pm 300$ & $6200 \pm 220$ & $5018 \pm 222$ & $6850 \pm 495$ \\
$35{ }^{\circ} \mathrm{C}$ & $1350 \pm 45$ & $3511 \pm 353$ & $2144 \pm 62$ & $3774 \pm 152$ \\
\hline
\end{tabular}

Figure 4.8 compares fouling resistances estimated by the UMFI method to the MFI-UF for various NOM foulants at different temperature conditions. From Figure 4.8, it can be observed that the MFI-UF values correlate well with the $\mathrm{UMFI}_{\mathrm{f}}\left(\mathrm{R}^{2}\right.$ of 0.76$)$ and $\mathrm{UMFI}_{\text {hir }}$ 
$\left(\mathrm{R}^{2}\right.$ of 0.86$)$. Since fouling irreversibility poses major implications during filtration with respect to membrane cleaning and life time, the MFI-UF testing method demonstrates a useful fit for predicting fouling before relying on operating data. Thus, a plant could use the MFI-UF to monitor and predict changes in irreversible fouling with temperature and make required alternations in membrane pretreatment and/or cleaning procedures.

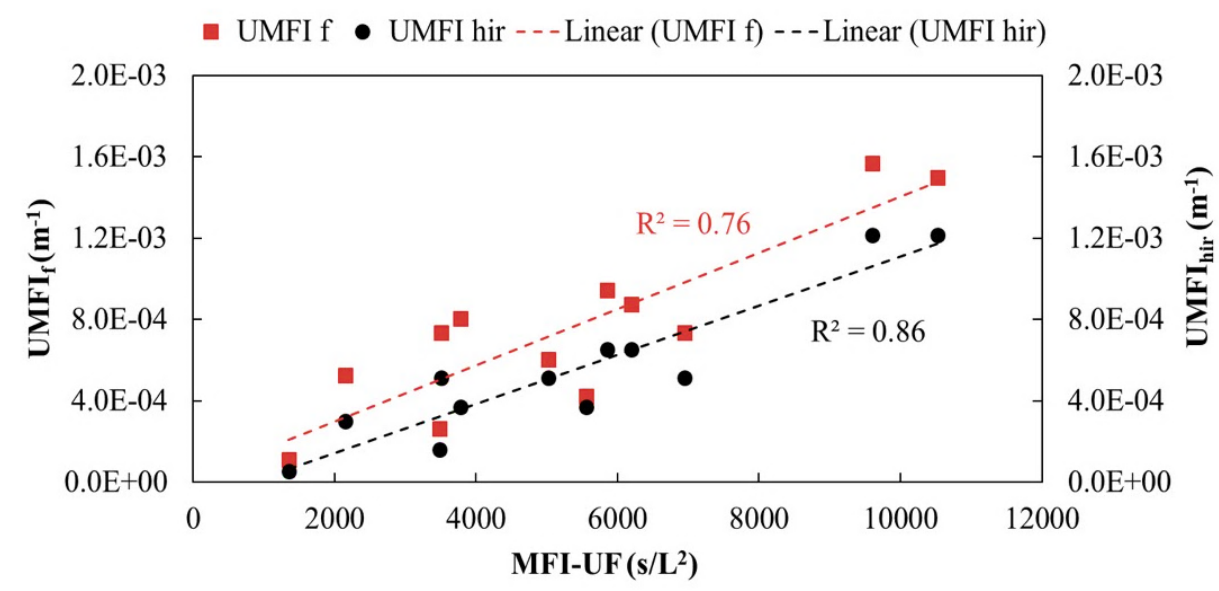

Figure 4.8 - Relationship between UMFI fouling resistances and MFI-UF fouling index

\subsubsection{Impact of water temperature on chemical cleaning}

As noted above, fouling with NOM across all model solutions increased as the water temperature decreased; additional tests were conducted to assess for changes in cleaning effectiveness with temperature as well. Figure 4.9 shows the ratio of chemically reversible fouling to hydraulically irreversible fouling indices, $\mathrm{UMFI}_{\mathrm{rr}} / \mathrm{UMFI}_{\text {hir }}$, (Figure 4.9A), and specific flux recoveries (Figure 4.9B) at different water temperatures. From Figure 4.9A, it can be clearly seen that the $\mathrm{UMFI} \mathrm{Ir}_{\mathrm{cr}} / \mathrm{UMFI}_{\text {hir }}$ ratios differ at different water temperatures. Under the operation at $5{ }^{\circ} \mathrm{C}$, chemical cleaning efficiency was the lowest and increased by 10-40\%, ( $\mathrm{p}<0.05$ ), with increasing filtration temperature from $5{ }^{\circ} \mathrm{C}$ to $20^{\circ} \mathrm{C}$. Thus, the 
initial chemical cleaning protocol recommended by the polymeric membrane's manufacturer (i.e. $\mathrm{NaOCl}$ followed by citric acid) was not sufficient in removing irreversible fouling at $5{ }^{\circ} \mathrm{C}$ compared to $20^{\circ} \mathrm{C}$. The decrease in the cleaning efficiency with temperature is attributed to the high irreversible fouling ratio at $5{ }^{\circ} \mathrm{C}$ as observed in Figure 4.7. Moreover, as water temperature increased from $20{ }^{\circ} \mathrm{C}$ to $35{ }^{\circ} \mathrm{C}$, the $\mathrm{UMFI}_{\mathrm{cr}} / \mathrm{UMFI}_{\text {hir }}$ ratios also increased but moderately by $10-15 \%(\mathrm{p}<0.05)$. The $\mathrm{UMFI}_{\mathrm{cr}} / \mathrm{UMFI}_{\text {hir }}$ ratios of the NOM mixture and BSA were always lower than that for alginate and humic acids, indicating lower cleaning efficiency for the NOM mixture and BSA. This attributes to the higher irreversible fouling observed with the NOM mixture and BSA compared with the alginate and humic acid waters. Clearly, a more aggressive chemical cleaning strategy is required to better remove NOM mixture and BSA fouling at the lower temperature, highlighting the need to focus on both NOM type and water temperature to optimize chemical cleaning regimes.

The influence of filtration temperature on NOM chemical cleaning can also be observed in Figure 4.9B. The specific flux recoveries at $5{ }^{\circ} \mathrm{C}$, after 1 cleaning cycle using $\mathrm{NaOCl}$ followed by citric acid, ranged from $85 \%$ to $95 \%$ which were significantly lower $(\mathrm{p}<0.05)$ than those obtained at $20^{\circ} \mathrm{C}$ (ranged from $95 \%$ to $98 \%$ ). The difference in the specific flux recovery at 5 and $20^{\circ} \mathrm{C}$ conditions is attributed to the differences in the irreversible fouling ratios as shown in Figure 4.7. Thus, a more aggressive cleaning was needed to recover specific flux at $5{ }^{\circ} \mathrm{C}$ compared to $20^{\circ} \mathrm{C}$. This can be supported by the specific flux recovery values at $35{ }^{\circ} \mathrm{C}$ in which all NOM types were effectively removed and the specific flux recovery was $>95 \%$. In addition, at $5{ }^{\circ} \mathrm{C}$, the NOM mixture, BSA, and alginate required 
an additional 1 hour cleaning using $\mathrm{NaOCl}$ (i.e. after the 1 recommended cleaning cycle using $\mathrm{NaOCl}$ followed by citric acid) to achieve $>95 \%$ of specific flux recovery (results not shown). On the other hand, at $20^{\circ} \mathrm{C}$ and $35^{\circ} \mathrm{C}$, membranes were able to achieve $>95 \%$ after only 1 cleaning cycle. Humic acid cleaning was less impacted by temperature variation compared to the other NOM models (refer to Figures 4.9A-B). These results indicate the need to emphasize good pretreatment steps or modification of cleaning steps during membrane operation in cold climates to remove the majority of NOM from membrane feed in order to ensure higher specific flux recovery.
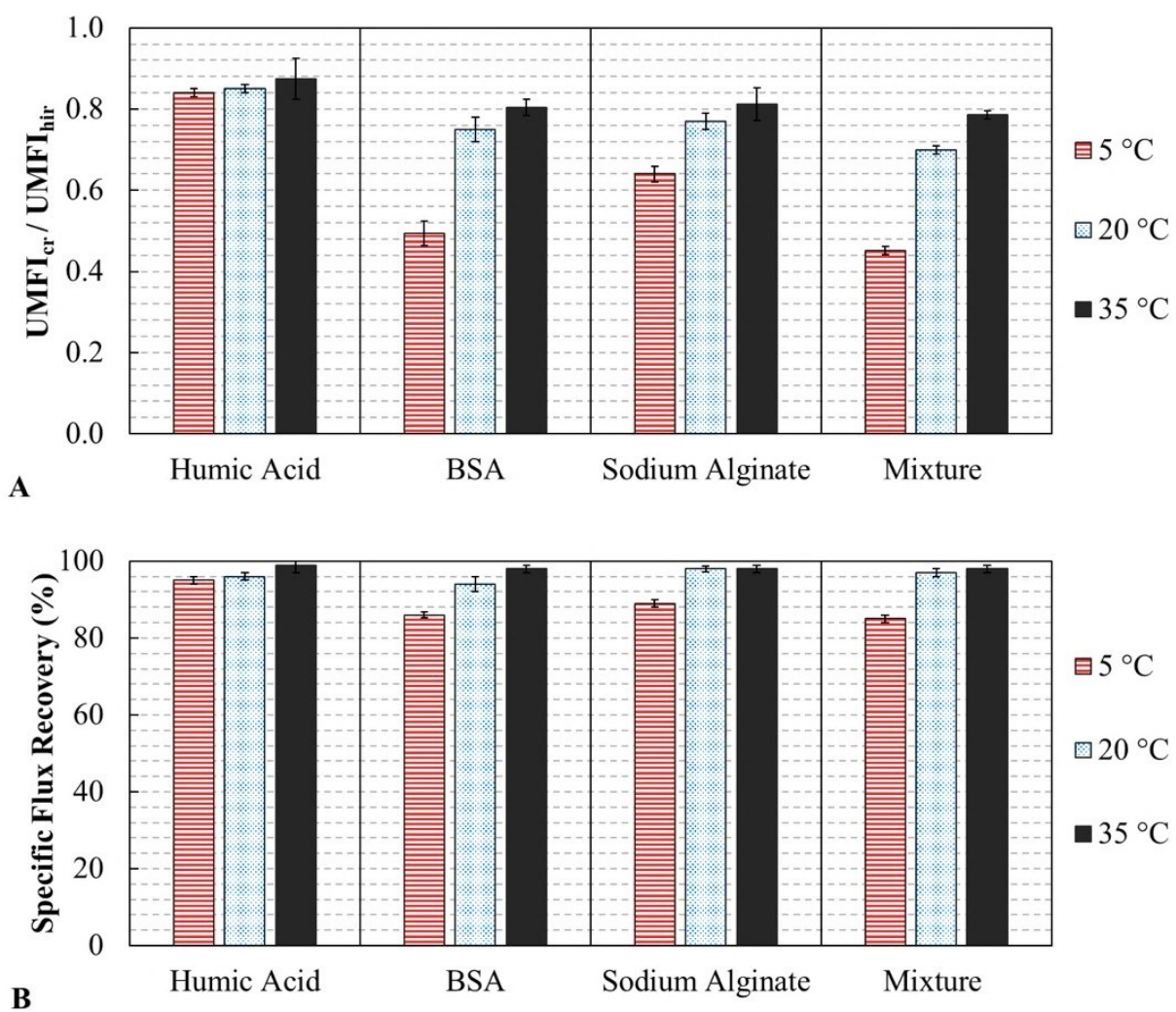

Figure 4.9 - Chemical cleaning efficiency at different filtration water temperature* . (A) $\mathrm{UMFI}_{\mathrm{cr}} / \mathrm{UMFI}_{\text {hir }}$ ratios**; (B) Specific flux recovery.

*After 1 cleaning cycle using $\mathrm{NaOCl}$ followed by citric acid.

**Note: The higher the $\mathrm{UMFI}_{\mathrm{cr}} / \mathrm{UMFI}_{\text {hir }}$ ratio the higher the chemical cleaning efficiency 


\subsection{Conclusions}

Limited research has investigated changes in NOM fouling and cleaning with temperature. This research investigated the impact of water temperature at $5^{\circ} \mathrm{C}, 20^{\circ} \mathrm{C}$ and $35^{\circ} \mathrm{C}$ on $\mathrm{NOM}$ fouling and cleaning using the UMFI and MFI-UF methods with the following key observations:

- Feed water temperature has a significant impact on submerged polymeric UF membrane fouling. Fouling increased by $15-35 \%$ when the water temperature decreased from $20{ }^{\circ} \mathrm{C}$ to $5{ }^{\circ} \mathrm{C}$ while the fouling decreased by $15-25 \%$ when the temperature increased to $35^{\circ} \mathrm{C}$. Thus, fouling analysis for plant subject to cold water conditions should be investigated more rigorously to help maintain membrane productivity.

- BSA was most sensitive to changes in water temperature whereas humic acid was least sensitive, highlighting the need to identify specific NOM fractions in water to better understand potential fouling changes.

- The MFI-UF fouling prediction trend was in strong agreement with UMFI indices for establishing NOM irreversible fouling, highlighting an advantage of the MFI-UF as a robust fouling prediction index for low pressure polymeric membranes.

- Aside from humic acid, chemical cleaning of all other NOM solutions was less efficient at the colder water temperature and thus indicate the need for alternative chemical cleaning strategies during lower water temperature conditions to recover membrane permeability. 


\subsection{Acknowledgements}

The authors would like to acknowledge the Saudi Arabia Ministry of Education (MOE) and Natural Sciences and Engineering Research Council of Canada (NSERC) for helping fund this research. The authors would also like to thank summer student, Bia Pereira, for her hard work and help in the laboratory.

\subsection{References}

Alresheedi, M., Basu, O., 2014. Support media impacts on humic acid, cellulose, and kaolin clay in reducing fouling in a submerged hollow fiber membrane system. Journal of Membrane Science 450, 282-290.

Alresheedi, M., Basu, O., 2017. Fouling indices and resistance in series methods for quantification of NOM fouling in submerged polymeric membrane systems. IWA Membrane Technology Conference, Singapore.

Alresheedi, M., Basu, O., 2018. Application of MFI-UF Fouling Index with NOM Fouling under Various Operating Conditions. Desalination and Water Treatment 133, 45-54.

ASTM International, Designation: D4189 - 07 (Reapproved 2014). Standard test method for silt density index (SDI) of water. 
Boerlage, S., Kennedy, M., Aniye, M., Abogrean, E., Tarawneh, Z., Schippers, J., 2003. The MFI-UF as a water quality test and monitor. Journal of Membrane Science 211, 271289.

Boerlage, S., Kennedy, M., Dickson, M., El-Hodali, D., Schippers, J., 2002. The modified fouling index using ultrafiltration membranes (MFI-UF): characterisation, filtration mechanisms and proposed reference membrane. Journal of Membrane Science 197, 1-21.

Chellam, S., Wendong, X., 2006. Blocking laws analysis of dead-end constant flux microfiltration of compressible cakes. Journal Colloid Science 301, 248-257.

Contreras, A., Kim, A., Li, Q., 2009. Combined fouling of nanofiltration membranes: mechanisms and effect of organic matter. Journal of Membrane Science 327, 87-95.

Crittenden, J., Trussell, R., Hand, D., Howe, K., Tchobanoglous, G., 2005. Water treatment: principles and design, 2nd edition, Ed, Wiley, New Jersey, USA.

De Souza, N., Basu, O., 2013. Comparative analysis of physical cleaning operations for fouling control of hollow fiber membranes in drinking water treatment. Journal of Membrane Science 436, 28-35. 
Farahbakhsh, K., Smith, D., 2006. Membrane filtration for cold regions - impact of cold water on membrane integrity testing. Journal of Environmental Engineering Science 5, 6975.

Foley, G., 2006. A review of factors affecting filter cake properties in dead-end microfiltration of microbial suspensions. Journal of Membrane Science 274, 38-46.

Gao, W., Qu, X., Leung, K., Liao, B., 2012. Influence of temperature and temperature shock on sludge properties, cake layer structure, and membrane fouling in a submerged anaerobic membrane bioreactor. Journal of Membrane Science 421, 131-144.

Hashino, M., Hirami, K., Katagiri, M., Kubota, T., 2011. Effects of three natural organic matter types on cellulose acetate butyrate microfiltration membrane fouling. Journal of Membrane Science 379, 233- 238.

Hermia, J., 1982. Constant pressure blocking filtration laws application to power-law non-newtonian fluids. Institution of Chemical Engineers 60, 183-187.

Huang, H., Young, T., Jacangelo, J., 2008. Unified membrane fouling index for low pressure membrane filtration of natural waters: Principles and methodology. Environmental Science and Technology 42, 714-720. 
Jeong, S., Vigneswaran, S., 2015. Practical use of standard pore blocking index as an indicator of biofouling potential in seawater desalination. Desalination 365, 8-14.

Jermann, D., Pronk, W., Boller, M., 2008. Mutual influences between natural organic matter and inorganic particles and their combined effect on ultrafiltration membrane fouling. Environmental Science and Technology 42, 9129-9136.

Jin, X., Jawor, A., Kim, S., Hoek, E., 2009. Effects of feed water temperature on separation performance and organic fouling of brackish water RO membranes. Desalination 239, 346359.

Katsoufidou, K., Sioutopoulos, D., Yiantsios, S., Karabelas, A., 2010. UF membrane fouling by mixtures of humic acids and sodium alginate: Fouling mechanisms and reversibility. Desalination, 264, 220-227.

Kertész, S., László, Z., Horváth, Z., Hodúr, C., 2008. Analysis of nanofiltration parameters of removal of an anionic detergent. Desalination 221, 303-311.

Kitis, M., Karanfil, T., Wigton, A., Kilduff, J., 2002. Probing reactivity of dissolved organic matter for disinfection by products formation using XAD-8 resin adsorption and UF fractionation. Water Research 36, 3834-3848.

Ma, Z., Wen, X., Zhao, F., Xia, Y., Huang, X., Wait, D., Guan, J., 2013. Effect of 
temperature variation on membrane fouling and microbial community structure in membrane bioreactor. Bioresources Technology 133, 462-468.

Puspitasari, V., Granville, A., Le-Clech, P., Chena, V., 2010. Cleaning and ageing effect of sodium hypochlorite on polyvinylidene fluoride (PVDF) membrane. Separation and Purification Technology 72, 301-308.

Schippers, J., Verdouw, J., 1980. The modified fouling index: a method of determining the fouling characteristics of water. Desalination 32, 137-148.

Sioutopoulos, D., Yiantsios, S., Karabelas, A., 2010. Relation between fouling characteristics of RO \& UF membranes in experiments with colloidal organic and inorganic species. Journal of Membrane Science 350, 62-82.

Steinhauer, T., Hanely, S., Bogendorfer, K., Kulozik, U., 2015. Temperature dependent membrane fouling during filtration of whey and whey proteins. Journal of Membrane Science 492, 364-370.

Taheri, A., Sim, L., Haur, C., Alkhondi, E., Fane, A., 2013. The fouling potential of colloidal silica and humic acid and their mixtures. Journal of Membrane Sceince 433, $112-$ 120. 
Villacorte, L., Ekowati, Y., Winters, H., Amy, G., Schippers, J., Kennedy, M., 2015. MF/UF rejection and fouling potential of algal organic matter from bloom-forming marine and freshwater algae. Desalination 367, 1-10.

Xiao, K., Wang, X., Huang, X., Waite, T., Wen, X., 2009. Analysis of polysaccharide, protein and humic acid retention by microfiltration membranes using Thomas' dynamic adsorption model. Journal of Membrane Science 342, 22-34.

Zhao, S., Zou, L., 2011. Effects of working temperature on separation performance, membrane scaling and cleaning in forward osmosis desalination. Desalination 278, 157164. 


\title{
Chapter 5
}

\section{Effects of Feed Water Temperature on Irreversible Fouling of Ceramic Ultrafiltration Membranes}

\author{
Mohammad T. Alresheedi ${ }^{*}$ and Onita D. Basu* \\ "Department of Civil and Environmental Engineering, Carleton University, 1125 Colonel \\ By Drive, Ottawa, ON, K1S 5B6, Canada. \\ Submitted to: Journal of Separation and Purification Technology
}

\begin{abstract}
Temperature is known to influence the filtration performance of membrane systems through its direct impact on water viscosity. This research demonstrates that the changes in natural organic matter (NOM) fouling behavior with temperature are over and beyond simple viscosity changes in water. Constant flux experiments were performed in a tubular ceramic ultrafiltration (UF) system at 5,20 , and $35^{\circ} \mathrm{C}$. The unified membrane fouling index (UMFI) was used to identify NOM reversible and irreversible fouling mechanisms; while the modified UF fouling index (MFI-UF) was used to predict the fouling potential of NOM. The results showed that after correcting for viscosity to standard $20{ }^{\circ} \mathrm{C}$ compared to $5{ }^{\circ} \mathrm{C}$, UMFI values were higher than expected and reflected the higher fouling irreversibility observed at the lower temperature. The lower water temperature resulted in an increase in NOM retention along with decrease in backwash and chemical cleaning effectiveness as determined by the UMFI and FEEM analyses. However, increased water temperature did not adversely impact existing backwash or chemical cleaning protocols. In addition, The MFI-UF exhibited the same trend as UMFI for establishing NOM fouling and retention,
\end{abstract}


and therefore, the MFI-UF method is suitable for use as fouling predictor with ceramic membrane systems.

Keywords: Ceramic ultrafiltration; Fouling indices; Irreversible fouling; Temperature

\subsection{Introduction}

Ceramic membranes are drawing increasing attention in drinking water treatment sectors as a cost competitive alternative to polymeric membranes (Samaei et al., 2018; Lee et al., 2014; Vasanth et al., 2013). This emerging technology takes advantage of superior chemical resistance that enables aggressive chemical cleaning and structural rigidity that alleviates problems associated with repetitive testing, repair, and replacement. However, similar to polymerics, ceramic membranes face several challenging issues. The most important of these is loss of flux as the result of fouling, which can cause major limitations in the performance of membranes (Chang et al., 2015). Membrane fouling involves the cumulative aggregation of different foulants such as NOM, inorganic substances, colloidal species, and microorganisms on membrane surfaces or within their matrices, which can degrade their performance as well as decrease longevity (Cui et al., 2017). Among these foulants, the presence of NOM, such as humic, protein, and polysaccharide substances, is considered one of the major foulants that causes both reversible and irreversible fouling of membranes in water filtration (Chang et al., 2015; Hashino et al., 2011; Katsoufidou et al., 2007). Irreversible fouling is more problematic, necessitating chemical cleaning. However, limiting or avoiding chemical cleaning is recommended because of the detrimental effects of excess chemicals on membrane lifetime and the additional operational costs (Chang et al., 2015). 
Many factors, including raw water characteristics, operational and environmental conditions (i.e., water temperature and $\mathrm{pH}$ ), can negatively impact membrane performance and fouling (Cui et al., 2017; Lee et al., 2014). Among these factors, feed water temperature is an important operating parameter that has effects on membrane filtration, due to the nature of seasonal changes in the temperature of raw water. Temperature has a direct impact on water density and viscosity, which influence membrane flux. As viscosity and density increase, the transmembrane pressure (TMP) that is required to pass the water through the membrane also increases, which results in a decrease in membrane permeability. However, the effect of temperature on water properties is not sufficient to fully explain the drop in membrane flux and permeability and often overlooked in the study of membrane fouling.

Previous studies addressing the impacts of feed water temperature with membranes were commonly done with high pressure nanofiltration (NF) and reverse osmosis (RO) membrane systems (Roy et al., 2017; Zhao et al., 2011; Jin et al., 2009) or membrane bio reactor (MBR) systems (Ma et al., 2013; Van den Brink et al., 2011) and more focused on inorganic scaling and biofouling challenges with operation at temperatures greater than 20 ${ }^{\circ} \mathrm{C}$ but not changes in reversible and irreversible NOM fouling behavior. In fact, limited studies exist that examine the impact of temperature below $20{ }^{\circ} \mathrm{C}$ with low pressure membranes (Stade et al., 2015; Steinhauer et al., 2015; Farahbakhsh et al., 2006). Farahbakhsh et al. (2006) found that current standards for membrane integrity testing overestimated safety design values by not taking into account temperatures below $5{ }^{\circ} \mathrm{C}$. Thus, thousands of dollars are wasted each year in membrane filtration plants to replace 
damaged membranes, because the negative effects of cold water on membrane performance and fouling are often overlooked (Cui et al., 2017). Despite some studies that have showed the effect of different temperatures on membrane performance and permeability, to our best knowledge, no attention has been paid to the impacts on reversible and irreversible NOM fouling and cleaning mechanisms of low pressure membranes. In addition, while previous studies on temperature impact were focused on polymeric membranes, the emerging market of ceramic membranes for drinking water has received little attention in this area but is important for their implementation for drinking water treatment applications.

From a water quality testing perspective, fouling index methods are commonly used to quickly assess the fouling tendency of membrane feed water at the design stage and/or pretreatment requirements. The silt density index (SDI) and modified fouling index (MFI), using a $0.45 \mu \mathrm{m}$ membrane, are commonly utilized as fouling predictors and for quality control during membrane operation (ASTM 2014; Schippers et al., 1980). Boerlage et al. (2002) developed the MFI-UF, making use of a $13 \mathrm{kDa}$ hollow fiber ultrafiltration (UF) membrane to count for particles $<0.45 \mu \mathrm{m}$. Feed water having an MFI-UF $<3000 \mathrm{~s} / \mathrm{L}^{2}$ is equivalent to $\mathrm{SDI}<3 \% / \mathrm{min}$, which is considered acceptable for membrane feed to control fouling (Boerlage et al., 2002). A number of studies applied the MFI-UF for assessing the fouling propensity with RO membrane systems (Taheri et al., 2013; Jeong et al. 2015; Villacorte et al., 2015). These studies have focused on particulate, inorganic scaling and biofouling with RO systems. Furthermore, in these studies, flat sheet UF membranes were used for the MFI-UF testing instead of hollow fiber membranes. In fact, the usability of the 
MFI-UF with the hollow fiber membrane method for NOM fouling under changes in water temperature conditions and its applicability with ceramic membranes has yet to be examined.

The unified membrane fouling index (UMFI) model (Huang et al., 2008) is commonly used to quantify fouling based on reversibility and irreversibility components. Thus, unlike the MFI-UF, the UMFI provides information on the current fouling a membrane is subjected to. The actual capacity of the MFI-UF and UMFI to be effectively used as fouling assessment methods with ceramic membranes has yet to be evaluated in general and under changing water temperature conditions. In this research, the effect of feed water temperature $\left(5,20\right.$, and $\left.35^{\circ} \mathrm{C}\right)$ on the filtration performance, irreversible fouling and chemical cleaning of a ceramic UF membrane were examined. The MFI-UF method was used to assess its effectiveness for predicting NOM fouling while the UMFI method was utilized to assess for respective changes in reversible and irreversible fouling ratios under changing water temperature conditions.

\subsection{Materials and Methods}

\subsubsection{Feed water solution}

A synthetic feed water solution comprised of a mixture of three different NOM components: humic substances (humic acids), protein (bovine serum albumin, BSA); and polysaccharide (sodium alginate) was used for this study. These NOM model components are commonly found in surface water and have been previously used in membrane fouling studies (Chang et al., 2015; Hashino et al., 2011; Katsoufidou et al., 2007). The total 
organic carbon (TOC) concentration in the feed water was $2.5 \mathrm{mg} \mathrm{C} / \mathrm{L}$ (i.e. $0.83 \mathrm{mg} \mathrm{C} / \mathrm{L} /$ each NOM model) and was chosen to simulate the organic matter content in surface water (Chang et al., 2015). A moderate hardness of $75 \mathrm{mg} / \mathrm{L}$ calcium carbonate $\left(\mathrm{CaCO}_{3}\right)$ and a low level of turbidity (5 NTU) as kaolin clay particles were included in the synthetic water matric to represent the more complex conditions of a surface water source (Lee et al., 2014; Jermann et al., 2008). All chemicals were purchased from Sigma Aldrich. Feed water solutions were prepared in DI water and were mixed using a magnetic stirrer one-day prior any experiment to ensure that materials were dissolved completely. Feed water was continuously mixed using a VWR dual speed mixer to ensure homogeneous water conditions throughout the experiment. The $\mathrm{pH}$ of the feed was adjusted as needed to 7.5 with $\mathrm{NaOH}$.

\subsubsection{Ceramic UF fouling and cleaning experiments}

Experiments were conducted under constant flux condition with an automated filtration system (Figure 5.1). Labview program code (National Instruments, NI) was created to operate the system automatically such as controlling the feed pump to have a constant flux, and automatically switches from filtration cycles to backwash cycles while recording flux and pressure data. Detailed information of the ceramic UF setup and equipment is described by Alresheedi et al. (2019). A tubular ceramic UF membrane $\left(\right.$ Ceramem $^{\mathrm{TM}}$, Veolia) was used and its characteristics are as follows: monolithic module, SiC membrane material, inside-out channels (61), square channel $(2 \mathrm{~mm})$, pore size $(0.01 \mu \mathrm{m})$, area $\left(0.1 \mathrm{~m}^{2}\right)$, and module length $(300 \mathrm{~mm})$. 


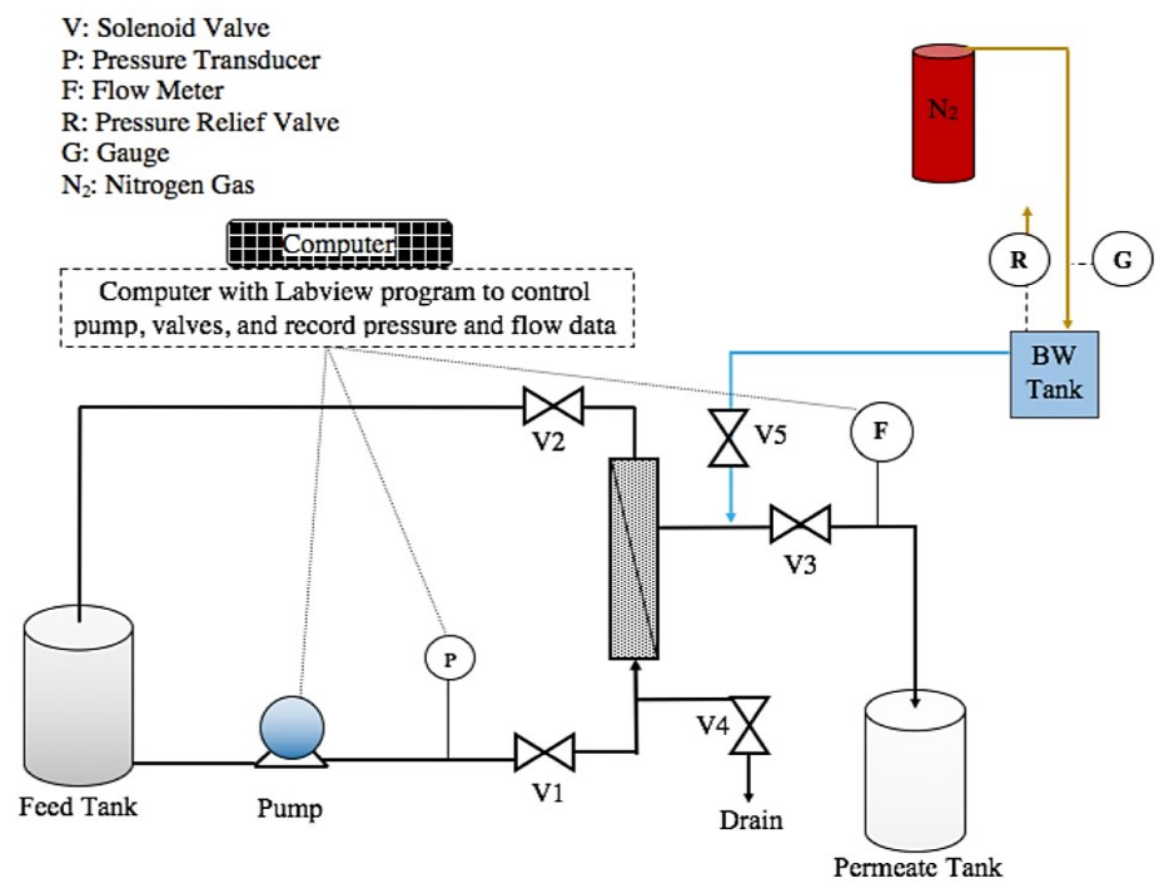

Figure 5.1 - A schematic representation of the ceramic UF setup

The ceramic membrane was fouled for 24 hours in dead-end filtration mode under constant flux of $100 \mathrm{LMH}$; backpulses were conducted for 20 seconds every 4 hours $\left(0.4 \mathrm{~m} / \mathrm{m}^{2}\right)$ using pressurized DI water at 2 bars ( $\sim 30 \mathrm{psig})$ at room temperature. To assess the potential seasonal impacts of water temperature on ceramic UF fouling and cleaning, feed water temperature was assessed at 5,20 , and $35{ }^{\circ} \mathrm{C}$. Temperature was controlled either using an immersion heater (Cole Palmer) or a compact chiller (LM series, Polyscience). The feed tank was insulated to maintain constant temperature throughout the testing period. Temperature were monitored using $\mathrm{HACH}$ temperature probe (cat.no. 58258-00) HQd Field Case equipment. Equation 5.1 (Crittenden et al., 2005) was used to correct flux measured at different temperature to standard temperature of $20{ }^{\circ} \mathrm{C}$. Experiments were performed in triplicates for each testing condition. 
$\mathrm{J}_{20}{ }^{\circ} \mathrm{C}=\mathrm{J}_{\mathrm{m}}(1.03)^{\mathrm{T}} 20^{\circ} \mathrm{C}^{-\mathrm{T}} \mathrm{m} \quad$ Equation (5.1)

$\mathrm{J}_{20}{ }^{\circ} \mathrm{C}$ and $\mathrm{T}_{20}{ }^{\circ} \mathrm{C}$ are the flux and temperature at $20{ }^{\circ} \mathrm{C}$, respectively. $\mathrm{J}_{\mathrm{m}}$ and $\mathrm{T}_{\mathrm{m}}$ are the measured flux and temperature respectively. After each fouling experiment, chemical cleaning (Clean in place, CIP) was performed according to the manufacturer recommendations using an oxidant, sodium hypochlorite $(\mathrm{NaOCl}, 500 \mathrm{mg} \mathrm{Cl} / 2 / \mathrm{L})$ followed by citric acid. Each cleaning step was performed for 4 hours. CIP wash water chemicals were prepared in $4 \mathrm{~L}$ of DI water and were recirculated for 1 hour followed by 3 hours soak. A clean water flux (CWF) test was then performed to determine the initial membrane resistance using the TMP versus flux relationship for a clean membrane.

\subsubsection{Analysis}

5.2.3.1 The unified membrane fouling index (UMFI)

To assess ceramic membrane fouling and cleaning performance, the UMFI model, Equation 5.2 (Huang et al., 2008) was applied to quantify each contributing fouling resistance.

$\frac{1}{\mathrm{~J}_{\mathrm{s}}{ }^{\prime}}=1+(\mathrm{UMFI}) \mathrm{x} \mathrm{V}_{\mathrm{S}}$

Equation (5.2)

Where $\mathrm{J}_{\mathrm{s}}$ ' is the normalized specific flux (i.e. measured specific flux $\left(\mathrm{J}_{\mathrm{s}}\right)$ divided by initial specific flux $\left(\mathrm{J}_{\mathrm{s} 0}\right)$, (unitless), UMFI is an estimate of the extent of fouling $\left(\mathrm{m}^{-1}\right)$, and $\mathrm{V}_{\mathrm{S}}$ $\left(\mathrm{m}^{3} / \mathrm{m}^{2}\right)$ is the specific permeate volume. Total fouling index $\left(\mathrm{UMFI}_{\mathrm{f}}\right)$ was calculated by using data from the entire filtration cycle (i.e., using the slope of the linear region of $1 / \mathrm{J}_{\mathrm{s}}$ ' 
versus $\mathrm{V}_{\mathrm{s}}$ graph from the start to end of filtration. Using the procedure described in greater detail by Huang et al. (2008), hydraulically irreversible fouling index $\left(\mathrm{UMFI}_{\text {hir }}\right)$, that was remained after backwash, and chemically irreversible fouling index (UMFI ${ }_{\text {cir }}$ ), that was remained after chemical cleaning, were also calculated using data collected after the hydraulic backwash and after the CIP procedure, respectively. Hydraulically reversible fouling index (UMFI $\mathrm{hr}_{\mathrm{hr}}$, that was removed by backwash, was calculated from the difference between $\mathrm{UMFI}_{\mathrm{f}}$ and $\mathrm{UMFI}_{\text {hir. }}$ Chemically reversible fouling index $\left(\mathrm{UMFI}_{\mathrm{cr}}\right)$, that was removed by chemical cleaning, was calculated from the difference between $\mathrm{UMFI}_{h i r}$ and $\mathrm{UMFI}_{\text {cir. }}$ Consequently, $\mathrm{UMFI}_{\mathrm{f}}$ was fractionated to reversible and irreversible fouling as described in Equation 5.3.

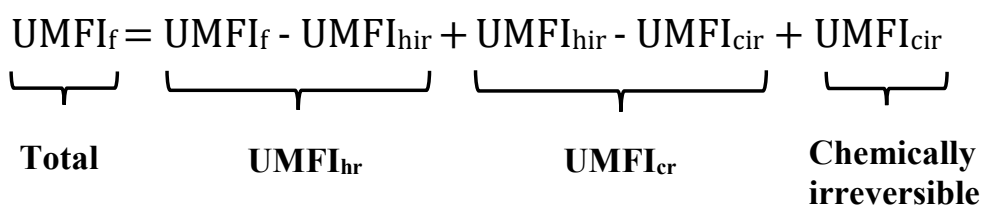

Changes in the backwash and chemical cleaning efficiencies with temperature were assessed using the hydraulically reversible to irreversible ratios $\left(\mathrm{UMFI}_{\mathrm{hr}} / \mathrm{UMFI}_{\text {hir }}\right)$ and chemically reversible to hydraulically irreversible ratios (UMFI $\left.\mathrm{Cr}_{\mathrm{rr}} / \mathrm{UMFI}_{\text {hir }}\right)$, respectively.

\subsubsection{Specific cake resistance}

The intrinsic resistance $\left(\mathrm{R}_{\mathrm{m}}\right)$ of a clean membrane was determined by filtering DI water through the ceramic membrane at a low flux $(\mathrm{J})$ and after recording the pressure $\left(\Delta \mathrm{P}_{0}\right)$ for 30 minutes, the flux was increased and the procedure continued in a stepwise fashion. Equation 5.4 was used to estimate $\mathrm{R}_{\mathrm{m} .} \mu$ is the water viscosity. 
$\mathrm{J}=\frac{\Delta \mathrm{P}_{0}}{\mu \mathrm{R}_{\mathrm{m}}}$

Equation (5.4)

For fouled membrane, assuming cake filtration is the dominant fouling mechanism, cake filtration mechanism can describe the pressure increase and membrane fouling. Equation 5.4 can be rewritten as:

$\mathrm{J}=\frac{\Delta \mathrm{P}}{\mu\left(\mathrm{R}_{\mathrm{C}}+\mathrm{R}_{\mathrm{m}}\right)}$

$\Delta \mathrm{P}$ is final transmembrane pressure $(\mathrm{kPa}) ; \mathrm{R}_{\mathrm{c}}$ is the cake layer resistance $\left(\mathrm{m}^{-1}\right)$. The $\mathrm{R}_{\mathrm{c}}$ can be expressed in terms of the specific cake resistance $\alpha c(\mathrm{~m} / \mathrm{kg})$, the bulk concentration, $\mathrm{C}_{\mathrm{b}}$ $\left(\mathrm{kg} / \mathrm{m}^{3}\right)$, and the specific permeate volume $\left(\mathrm{V}_{\mathrm{s}}\right)$, as follows:

$\mathrm{Rc}=\alpha_{\mathrm{c}} \mathrm{C}_{\mathrm{b}} \mathrm{V}_{\mathrm{S}} \quad$ Equation (5.6)

Equation 5.7 (Chellam et al., 2006) can be used to express the increase in pressure at constant flux during cake filtration:

$\Delta \mathrm{P}=(\mathrm{J} \cdot \mu)\left(\alpha_{\mathrm{c}} \cdot \mathrm{C}_{\mathrm{b}} \cdot \mathrm{V}_{\mathrm{S}}+\mathrm{R}_{\mathrm{m}}\right)=\Delta \mathrm{P}_{0}+\left(\mathrm{J} \cdot \mu \cdot \mathrm{C}_{\mathrm{b}} \cdot \mathrm{V}_{\mathrm{S}}\right) \alpha_{\mathrm{c}} \quad$ Equation (5.7)

5.2.3.3 Carbon and fluorescence excitation and emission matrix (FEEM) analyses

NOM retention at different water temperature conditions was determined by performing total organic carbon (TOC) analysis (using a Shimadzu TOC-VCPH/ CPN analyzer) on samples from feed and permeate water. NOM retention was then calculated using Equation 5.8 .

NOM Retention $=\left(\frac{\text { Feed }_{C}-\text { Permeate }_{C}}{\text { Feed }_{C}}\right) \times 100 \quad$ Equation (5.8) 
Where Feed $_{C}$ is the carbon concentration in feed water; Permeate $\mathrm{C}_{\mathrm{C}}$ is the carbon concentration in permeate water. Multiple TOC samples were taking during filtration time and the average TOC values were used to calculate the percentage NOM retention.

A carbon mass balance was performed to determine indirectly the carbon mass remaining on the membrane before and after backwashing and chemical cleaning. This was done by performing TOC analysis on feed, permeate, hydraulic backwash and chemical wash waters. The mass of hydraulically irreversible carbon, which refer to the foulants remained on the membrane after filtration and before chemical cleaning, was determined using Equations 5.9. The hydraulically irreversible carbon was further divided into chemically reversible and irreversible, which refer to the foulants removed by chemical cleaning and foulants remained on the membrane after chemical cleaning, respectively (as shown in Equation 5.10).

$$
\begin{aligned}
& \left(C_{\text {hir. }} V_{\text {hir }}\right)=\left(\mathrm{C}_{\mathrm{f} .} \mathrm{V}_{\mathrm{f}}\right)-\left(\mathrm{C}_{\mathrm{p}} \mathrm{V}_{\mathrm{p}}\right)-\left(\mathrm{C}_{\mathrm{hr}} \cdot \mathrm{Vh}_{\mathrm{hr}}\right) \quad \text { Equation }(5.9) \\
& \left(\mathrm{C}_{\text {hir. }} \mathrm{V}_{\text {hir }}\right)=\left(\mathrm{C}_{\mathrm{cr}} \cdot \mathrm{V}_{\mathrm{cr}}\right)+\mathrm{M}_{\mathrm{cir}} \quad \text { Equation (5.10) }
\end{aligned}
$$

Where: $\mathrm{C}(\mathrm{mg} \mathrm{C} / \mathrm{L})$ is the carbon concentration; $\mathrm{V}(\mathrm{L})$ is the water volume; $\mathrm{f}, \mathrm{p}, \mathrm{hr}$, hir, cr, and $\mathrm{M}_{\mathrm{cir}}$ denote feed water, permeate, hydraulically reversible (i.e. in backwash water), hydraulically irreversible (i.e. remained on membrane after backwash and before chemical cleaning), chemically reversible (i.e. in chemical wash water), and chemically irreversible (i.e. remained on membrane after chemical cleaning), respectively. The percentage error of the carbon measurements was calculated to ensure accurate mass balance at different 
testing conditions. Backwash and chemical cleaning efficiencies were assessed using the hydraulically reversible to irreversible carbon mass ratios $\left(\mathrm{C}_{\mathrm{hr}} \cdot \mathrm{V}_{\mathrm{hr}} / \mathrm{C}_{\mathrm{hir}} . \mathrm{V}_{\mathrm{hir}}\right)$ and chemically reversible to hydraulically irreversible carbon mass ratios $\left(\mathrm{C}_{\mathrm{cr}} . \mathrm{V}_{\mathrm{cr}} / \mathrm{C}_{\text {hir }} . \mathrm{V}_{\text {hir }}\right)$, respectively. Carbon data was then compared to the fouling indices data estimated by the UMFI method.

Additionally, FEEM analysis was performed on water samples from feed, permeate, backwash, and chemical wash waters for qualitative insights into NOM fouling and cleaning at different temperature conditions examined. For each sample, the fluorescence was measured by scanning the excitation wavelengths from 250 to $350 \mathrm{~nm}$ in $2 \mathrm{~nm}$ steps and detecting the emission intensity in $10 \mathrm{~nm}$ steps between 250 and $600 \mathrm{~nm}$ using Cary Eclipse Fluorescence Spectrophotometer (Varian, Surrey, UK). Excitation and emission slit widths were $5 \mathrm{~nm}$. Each measurement was carried out in standard quartz cuvette (1.0 cm path length).

\subsubsection{MFI-UF fouling index}

Fouling potential of the NOM mixture solution from the ceramic UF membrane experiments was assessed using the MFI-UF method (Boerlage et al., 2002). The MFI-UF

setup is described in detail by Boerlage et al. (2002) and Alresheedi et al. (2018). A hollow fiber polymeric UF membrane was used for the MFI-UF testing (proposed by Boerlage et al., 2002), with the following characteristics: molecular weight cut off (13 kDa); membrane materials (PAN); inside out; fiber inside diameter $(0.8 \mathrm{~mm})$; fiber outside diameter $(1.4$ 
$\mathrm{mm})$; number of fibers (400); membrane area $\left(0.2 \mathrm{~m}^{2}\right)$; module length (347 mm); module diameter (42 mm).

MFI-UF testing was determined by filtering feed water through the hollow fiber UF membrane under dead-end mode and constant pressure of 2 bars. Permeate water was collected in a tank set on an electronic balance to acquire permeate volume (V) and filtration time $(\mathrm{t})$ data from the balance. The MFI-UF was then calculated using Equation 5.11 (rewritten in Equation 5.12) (Boerlage et al., 2002). Where t is the filtration time (s); $\mathrm{V}$ is permeate produced $\left(\mathrm{m}^{3}\right) ; \Delta \mathrm{P}$ is the pressure (bar); $\mu$ is the viscosity of water $(\mathrm{kg} / \mathrm{m} \mathrm{s})$; A is the membrane area $\left(\mathrm{m}^{2}\right) ; \mathrm{R}_{\mathrm{m}}$ is the membrane resistance $\left(\mathrm{m}^{-1}\right) ; \alpha_{\mathrm{c}}$ is the specific cake resistance $(\mathrm{m} / \mathrm{kg})$; and $\mathrm{C}_{\mathrm{b}}$ is the bulk concentration $\left(\mathrm{kg} / \mathrm{m}^{3}\right) . \mathrm{d}(\mathrm{t} / \mathrm{V}) / \mathrm{dV}$ is the slope of two data points in the linear region (cake filtration region) of $t / V$ versus $V$ graph (described in detail by Alresheedi et al. 2018; Boerlage et al. 2002). The MFI-UF test were performed under 5,20 , and $35^{\circ} \mathrm{C}$ water temperature.

$\frac{\mathrm{t}}{\mathrm{V}}=\frac{\mu \mathrm{R}_{\mathrm{m}}}{\Delta \mathrm{PA}}+\left(\frac{\mu \mathrm{C}_{\mathrm{b}} \alpha_{\mathrm{c}}}{2 \Delta \mathrm{PA}^{2}}\right) \mathrm{V}=\frac{\mu \mathrm{R}_{\mathrm{m}}}{\Delta \mathrm{PA}}+\mathrm{MFI}-\mathrm{UF}(\mathrm{V})$

Equation (5.11)

Which can be rewritten as

MFI-UF $=\left(\frac{\mu C_{b} \alpha_{c}}{2 \Delta P A^{2}}\right)=\frac{d\left(\frac{t}{v}\right)}{d V}$

Equation (5.12)

\subsubsection{Statistical analysis}

Fouling and cleaning data obtained at different water temperature conditions were analyzed with the statistical program SPSS 22.0. One-way ANOVA (using the F-test) was performed 
on the data collected. The mean difference between conditions was considered to be significant at a $p$-value of $\leq 0.05$.

\subsection{Results and Discussion}

\subsubsection{Effect of feed water temperature on ceramic UF fouling}

Figure 5.2 illustrates the effect of water temperature variation on ceramic UF fouling. The data shown in Figures 5.2A and 5.2B are the experimental $\mathrm{UMFI}_{\mathrm{f}}\left(\mathrm{UMFI}_{\mathrm{f}}(\mathrm{exp})\right)$, which represents the $\mathrm{UMFI}_{\mathrm{f}}$ graph at temperature $(\mathrm{T})$, and the normalized $\mathrm{UMFI}_{\mathrm{f}}\left(\mathrm{UMFI}_{\mathrm{f}}(\right.$ nor $)$ ), which represents the $\mathrm{UMFI}_{\mathrm{f}}$ graph normalized to $20{ }^{\circ} \mathrm{C}$ to account for changes in water viscosity using Equation 5.1, respectively. Therefore, the difference in $\mathrm{UMFI}_{\mathrm{f}}$ (nor) values at different temperature is due to fouling. It can be clearly seen that with decreasing water temperature from $20{ }^{\circ} \mathrm{C}$ to $5{ }^{\circ} \mathrm{C}$, the $\mathrm{UMFI}_{\mathrm{f}}$ (exp) of the NOM mixture (Figure $5.2 \mathrm{~A}$ ) increased from $6.9 \mathrm{E}-01$ to $12.5 \mathrm{E}-01 \mathrm{~m}^{-1}$; while $\mathrm{UMFI}_{\mathrm{f}}(\exp )$ decreased from $6.9 \mathrm{E}-01$ to 4.6E- $01 \mathrm{~m}^{-1}$ when the temperature increased from $20^{\circ} \mathrm{C}$ to $35^{\circ} \mathrm{C}$. According to Darcy's law, the flux is a function of filtration pressure, membrane resistance, and the viscosity of water, therefore, water temperature has a great effect on flux because as both the membrane resistance and viscosity are temperature dependent. The increase in $\mathrm{UMFI}_{\mathrm{f}}(\exp )$ with decreasing temperature at constant flux filtration could be partially attributed to the increase in solution viscosity combined with increase in resistance of the membrane material, which then resulted in higher $\mathrm{UMFI}_{\mathrm{f}}(\exp )$ value (Cui et al., 2017; Lee et al., 2013). However, Figure 5.2B shows that the changes in NOM fouling with temperature are over and beyond simple viscosity changes of water. The $\mathrm{UMFI}_{\mathrm{f}}$ (nor) values (i.e. after correcting for viscosity) showed that at $5{ }^{\circ} \mathrm{C}$, the fouling index was $10.5 \mathrm{E}-01 \mathrm{~m}^{1}$ which was 
significantly higher than that at $20{ }^{\circ} \mathrm{C}\left(\mathrm{UMFI}_{\mathrm{f}}\right.$ (nor) of $\left.6.9 \mathrm{E}-01 \mathrm{~m}^{-1}\right)(\mathrm{p}<0.05)$; while the $\mathrm{UMFI}_{\mathrm{f}}$ (nor) value at $35^{\circ} \mathrm{C}$ decreased to $6.0 \mathrm{E}-01 \mathrm{~m}^{-1}$. Therefore, the increase or decrease in the $\mathrm{UMFI}_{\mathrm{f}}(\exp )$ values with temperature was not solely attributed to changes in water viscosity.
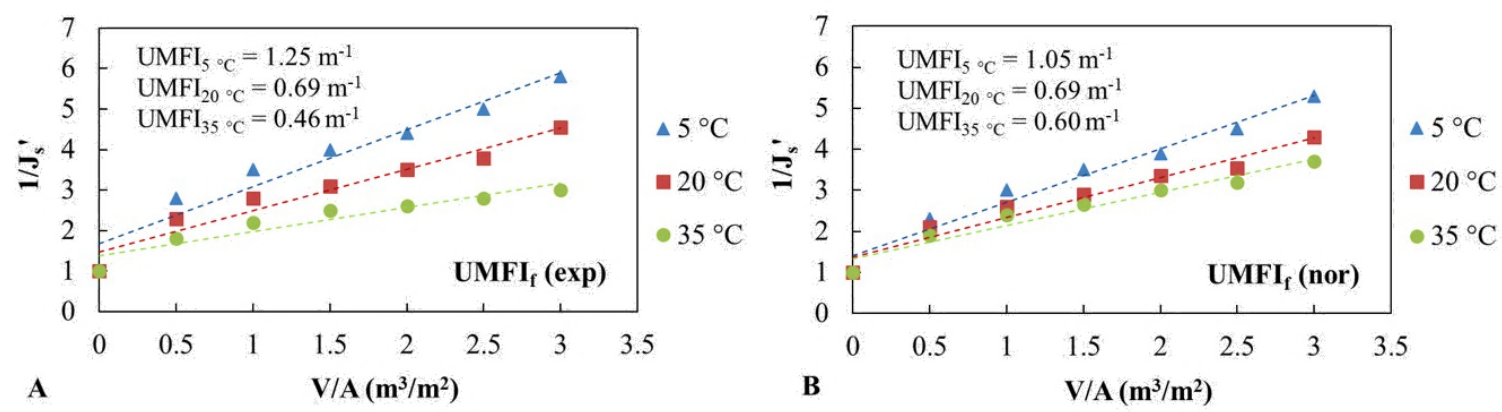

Figure 5.2 - Changes in the UMFI fouling index at different water temperature.

(A) $\mathrm{UMFI}_{\mathrm{f}}$ (exp): UMFI before viscosity correction; (B) UMFI (nor): UMFI after viscosity correction

Figure 5.3 shows the changes in the UMFI values due to viscosity changes versus fouling with temperature. The $\mathrm{UMFI}_{\mathrm{f}}$ (nor) values showed that at $5{ }^{\circ} \mathrm{C}$, only $43 \%$ of the increased in fouling index was attributed to the high viscosity of water; whereas $57 \%$ was due to fouling. Also, at $35{ }^{\circ} \mathrm{C}, 64 \%$ of the decreased in fouling index was attributed to the low viscosity of water whereas $36 \%$ was due to fouling. Thus, it is evident that the increase or decrease in the UMFI with temperature is due to changes in fouling behavior of NOM combined with changes in water viscosity. 


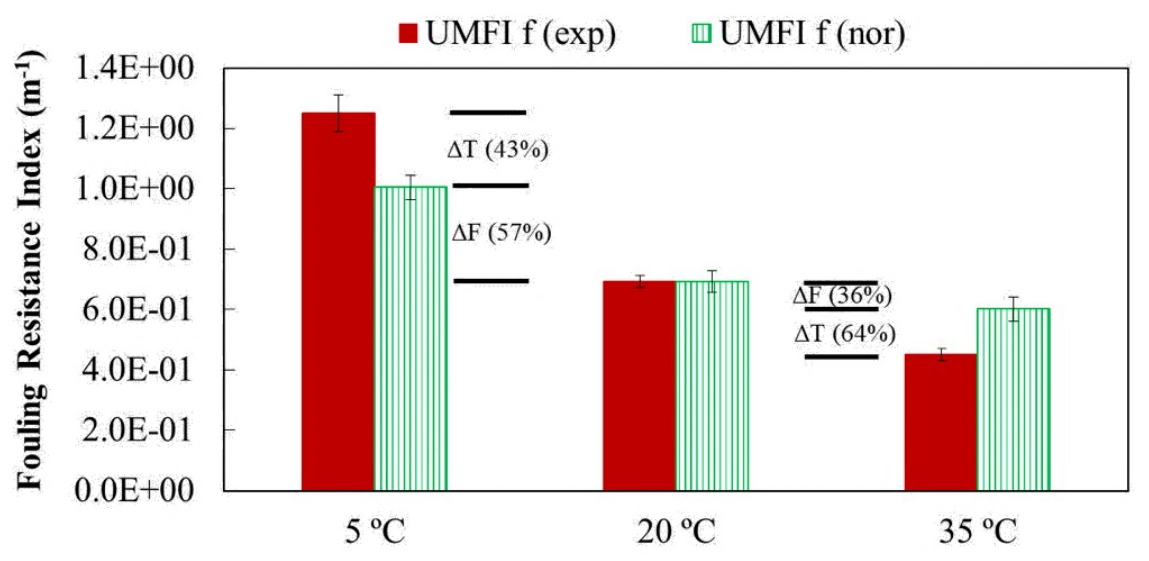

Figure 5.3 - Changes in $\mathrm{UMFI}_{\mathrm{f}}$ values due to changes in viscosity versus fouling with temperature*

*UMFI $\left(\exp\right.$ ): experimental unified membrane fouling index; $\mathrm{UMFI}_{\mathrm{f}}$ (nor): normalized unified membrane fouling index. $\Delta \mathrm{T}$ : changes in $\mathrm{UMFI}_{\mathrm{f}}$ due to viscosity changes; $\Delta \mathrm{F}$ : changes in $\mathrm{UMFI}_{\mathrm{f}}$ due to fouling

Additionally, Table 5.1 shows that the specific cake resistance, $\alpha_{c}$, value of the NOM mixture increased as feed water temperature decreased from 35 to $5{ }^{\circ} \mathrm{C}$. The higher $\alpha_{c}$ value at $5{ }^{\circ} \mathrm{C}$ indicates an increase in foulant deposition on the ceramic membrane and the production of a cake layer with lower permeability and higher fouling resistance. The percentage NOM retained by the ceramic UF increased from $62 \%$ to $88 \%(p<0.05)$ with decreasing temperature from $35^{\circ} \mathrm{C}$ to $5{ }^{\circ} \mathrm{C}$ (refer to Table 5.1) and consistent with the $\alpha_{c}$ findings. The increase in NOM retention at lower temperature could be attributed to the changes in the fouling layer formed on the membrane and increase in NOM size, thus, more NOM were retained by the ceramic UF membrane and higher fouling occurred. Cui et al. (2017) study reported a decrease in polyvinylidene fluoride (PVDF) membrane diameter and membrane permeability and an increase in the intrinsic hydraulic resistance were observed for a membrane operated at cold water temperature $\left(0.3^{\circ} \mathrm{C}\right)$. Similarly, Jin 
et al. (2009) reported higher NOM capture with RO systems in which the size of humic acid increased with decreasing water temperature. Therefore, temperature has an impact of both membrane properties and foulants.

Table 5.1- Estimated specific cake resistance and NOM retention values at different temperature $(n=3)$

\begin{tabular}{ccc}
\hline Water & $\begin{array}{c}\text { Specific Cake } \\
\text { Resistance }\left(\alpha_{\mathrm{c}}\right)\end{array}$ & $\begin{array}{c}\text { Retention } \\
(\%)\end{array}$ \\
\hline $5^{\circ} \mathrm{C}$ & 17.21 & $88 \pm 2$ \\
$20^{\circ} \mathrm{C}$ & 14.62 & $78 \pm 6$ \\
$35^{\circ} \mathrm{C}$ & 13.08 & $62 \pm 4$ \\
\hline
\end{tabular}

FEEM analysis (Figure 5.4) was used to analyze samples from the ceramic UF permeate water at two different temperature conditions $\left(5^{\circ} \mathrm{C}\right.$ and $\left.20^{\circ} \mathrm{C}\right)$. The FEEM analysis were used to provide qualitative information on NOM retention with temperature. Higher FEEM intensities indicate more NOM in the water, thus, lower permeate quality. The fluorescence peaks for humic-like substances are reported from 250-390 nm (range of excitation) and between 400-500 nm (range of emission). The fluorescence peaks for protein/polysaccharides-like substances are from 230-290 nm (range of excitation) and between 300-360 nm (range of emission) (Matilainen et al., 2011). In Figure 5.4, the fluorescence intensities/peaks of the NOM mixture permeate water at $5{ }^{\circ} \mathrm{C}$ were 18.2 au for humic acid and 21.7 au for proteins/alginate. At $20^{\circ} \mathrm{C}$, the FEEM intensities for humic acid in the permeate water increased to 25.6 au whereas much higher protein/alginate substances were found in the permeate water (38.6 au) indicating lower NOM retention at 
$20{ }^{\circ} \mathrm{C}$ compared to $5{ }^{\circ} \mathrm{C}$. Van den Brink et al. (2011) study with MBR systems found that with decreasing temperature, a shift was found in polysaccharides particle size distribution which led to high retention and fast pore blocking, thus, higher fouling at lower temperature. The FEEM results correlate well with the $\mathrm{UMFI}_{\mathrm{f}}$ values (Figure 5.3) and NOM retention data (Table 5.1) demonstrating the effect of temperature on NOM retention by the ceramic UF membrane and the quality of permeate water.
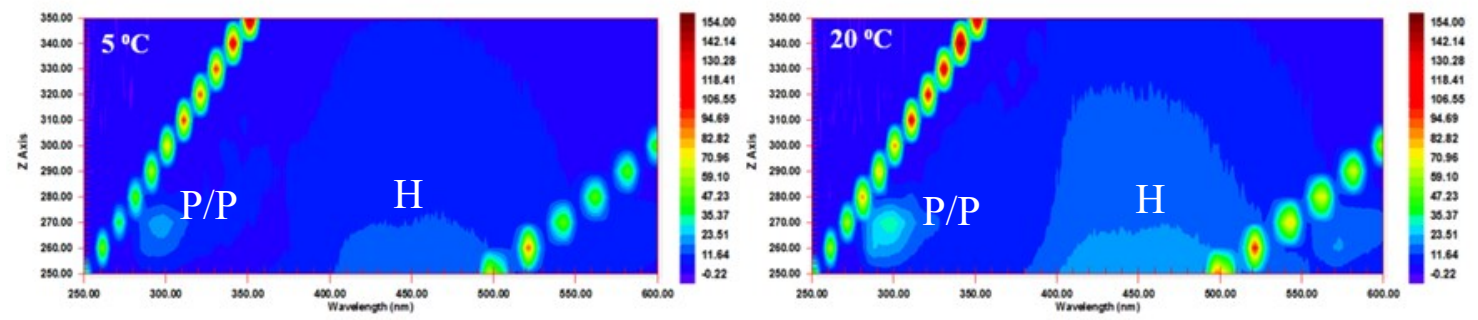

Figure 5.4 - FEEM images of the ceramic UF permeate of NOM mixture at 5 and $20{ }^{\circ} \mathrm{C}$. P/P: (proteins, BSA/ polysaccharides); H: (humics)

\subsubsection{Impact of feed water temperature on backwash and chemical cleaning} effectiveness

The overall UMFI analysis showed an impact of water temperature on NOM fouling; this can be further categorized by the reversible and irreversible fouling fractions to help assess backwash and chemical cleaning effectiveness. Figure 5.5 demonstrates that with decreasing temperature from $20^{\circ} \mathrm{C}$ to $5{ }^{\circ} \mathrm{C}$, the hydraulically irreversible fouling index $\left(\mathrm{UMFI}_{\text {hir }}\right)$ increased by $35 \%(\mathrm{p}<0.05)$, whereas the $\mathrm{UMFI}_{\text {hir }}$ decreased by $15 \%(\mathrm{p}<0.05)$, with increasing temperature from $20{ }^{\circ} \mathrm{C}$ to $35^{\circ} \mathrm{C}$. Thus, the increase in NOM fouling at lower temperature was due to the higher irreversible accumulation of NOM which resisted hydraulic backwashes. That is the temperature drop not only increases fouling due to 
hydraulic resistance with the increased water viscosity; but also the very nature of the NOM alters with the drop in water temperature and some fraction of the NOM shifts from reversible at $20 \mathrm{C}$ to irreversible at $5 \mathrm{C}$. This is reflected in the hydraulically reversible fouling index (UMFI $\mathrm{I}_{\mathrm{hr}}$ ) data in which the backwash efficiency was lower at $5{ }^{\circ} \mathrm{C}$ and increased as temperature increased from 5 to $35^{\circ} \mathrm{C}$. The chemically irreversible fouling index $\left(\mathrm{UMFI}_{\text {cir }}\right)$ was the highest at $5{ }^{\circ} \mathrm{C}$ indicating lower chemical cleaning efficiency at the lower temperature. Irreversibility of the foulant layer is a major concern during membrane filtration, as it influences physical and chemical cleaning requirements. The significant amount of hydraulically and chemically irreversible fouling at $5{ }^{\circ} \mathrm{C}$ pose a potential limitation to longer membrane operation.

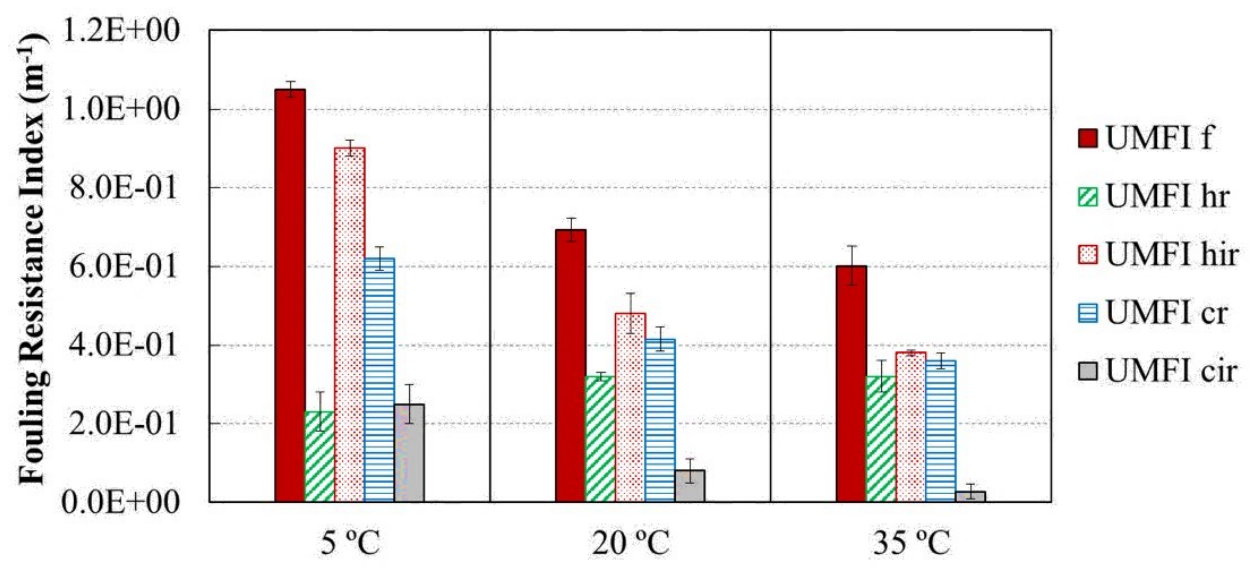

Figure 5.5 - UMFI fouling indices at different water temperature conditions*

*Note: UMFI $\mathrm{I}_{\mathrm{hr}}$ : unified hydraulically reversible fouling index; UMFI $\mathrm{Uhr}_{\text {ir: }}$ unified hydraulically irreversible fouling index $\mathrm{UMFI}_{\mathrm{cr}}$ : unified chemically reversible fouling index; $\mathrm{UMFI}_{\text {cir: }}$ unified chemically irreversible fouling index

Figure 5.6 shows the hydraulically reversible to irreversible fouling ratios $\left(\mathrm{UMFI}_{\mathrm{hr}} / \mathrm{UMFI}_{\mathrm{hir}}\right)$ and carbon ratios $\left(\mathrm{C}_{\mathrm{hr}} / \mathrm{C}_{\text {hir }}\right)$ at different temperature. With decreasing 
water temperature from $20{ }^{\circ} \mathrm{C}$ to $5{ }^{\circ} \mathrm{C}$, the $\mathrm{UMFI}_{\mathrm{hr}} / \mathrm{UMFI}_{\text {hir }}$ ratio of the NOM mixture decreased from 0.62 to $0.35(\mathrm{p}<0.05)$. The decreased in the $\mathrm{UMFI}_{\mathrm{hr}} / \mathrm{UMFI}_{\text {hir }}$ at lower temperature indicated lower hydraulic backwash efficiency, thus, higher NOM hydraulically irreversible fouling. This can be supported by the carbon data in which the $\mathrm{C}_{\mathrm{hr}} / \mathrm{C}_{\text {hir }}$ ratio decreased from 0.45 to 0.31 with decreasing temperature from $20^{\circ} \mathrm{C}$ to $5{ }^{\circ} \mathrm{C}$. Thus, the backwash pressure applied to mitigate fouling at standard feed water temperature of $20^{\circ} \mathrm{C}$ was not sufficient in removing NOM fouling deposits at $5{ }^{\circ} \mathrm{C}$. This is consistent with the increase in specific cake resistance and NOM retention values at lower temperature (refer to Table 5.1). The $\mathrm{UMFI}_{\mathrm{hr}} / \mathrm{UMFI}_{\mathrm{hir}}$ and $\mathrm{C}_{\mathrm{hr}} / \mathrm{C}_{\text {hir }}$ ratios at $35^{\circ} \mathrm{C}$ increased to 0.71 and 0.66 , respectively, indicating more effective backwash at higher temperature. This could be partially attributed to the lower cake resistance and NOM retention at $35^{\circ} \mathrm{C}$ (refer to Table 5.1), hence, better cleaning.

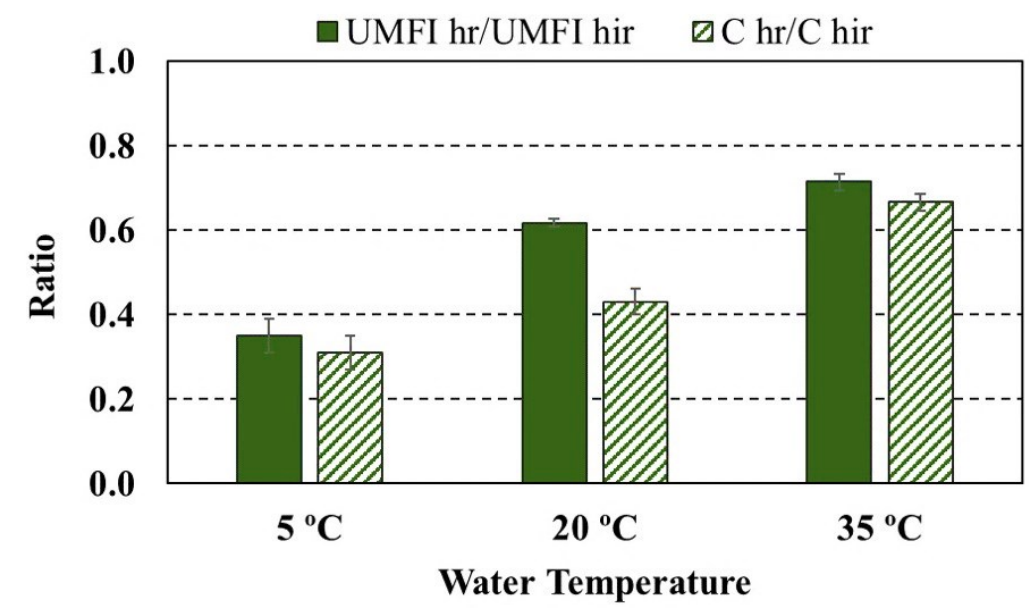

Figure 5.6 - Effect of water temperature on backwash efficiency* $\mathrm{UMFI}_{\mathrm{hr}} / \mathrm{UMFI}_{\mathrm{hir}}$ : hydraulically reversible to irreversible fouling ratios; $\mathrm{C}_{\mathrm{hr}} / \mathrm{C}_{\mathrm{hir}}$ : hydraulically reversible to irreversible carbon ratios.

*Note: The higher the $\mathrm{UMFI}_{\mathrm{hr}} / \mathrm{UMFI}_{\text {hir }}$ ratio, the higher the backwash efficiency; the higher the $\mathrm{C}_{\mathrm{hr}} / \mathrm{C}_{\mathrm{hir}}$ ratio, the higher the removed carbon mass 
The results from the UMFI analysis can be supported by the FEEM plots of the NOM mixture backwash water (Figure 5.7). At $20^{\circ} \mathrm{C}$, the fluorescence intensities/peaks for humic acid and proteins/alginate were 27.6 au and $37.1 \mathrm{au}$, respectively; while at $5{ }^{\circ} \mathrm{C}$, the fluorescence intensities/peaks for humic acid in the backwash water was 24.2 au and surprisingly no proteins/alginate were found in the backwash water at $5{ }^{\circ} \mathrm{C}$. By comparing the FEEM plots of the permeate water (Figure 5.4) and the backwash water (Figure 5.7) at $5{ }^{\circ} \mathrm{C}$, it is clear that proteins/alginate substances resisted hydraulic backwash and caused irreversible fouling for the ceramic UF. Van den Brink et al. (2011) and Ma et al. (2013) studies found that lower temperature had negative impacts on proteins and polysaccharides substances and biofouling in MBR systems. Therefore, both proteins and polysaccharides are problematic for membrane systems and more impacted by water temperature condition than humic substances. The FEEM plots correlate well with the UMFI $I_{h r} / \mathrm{UMFI}_{\text {hir }}$ ratios (Figure 5.6) demonstrating the effect of temperature on the NOM reversibility and irreversibility. Therefore, it is recommended to alter membrane backwash procedure with temperature (i.e. by increasing backwash pressure, frequency, etc.,) to better control membrane fouling.
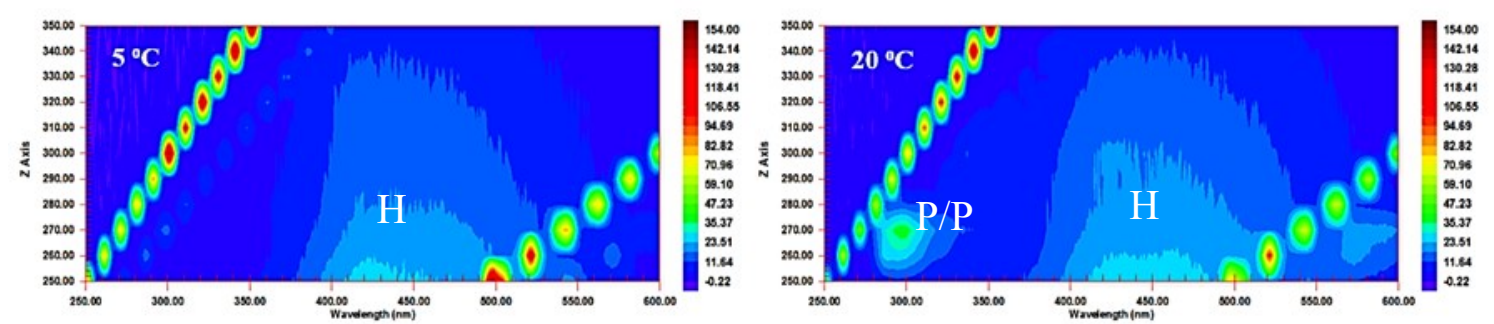

Figure 5.7 - FEEM images of the ceramic UF backwash water of NOM mixture at 5 and $20^{\circ} \mathrm{C}$. P/P: (proteins, BSA/ polysaccharides); H: (humics) 
Figure 5.8 shows the chemical cleaning CIP efficiency at different feed water temperature conditions. The $\mathrm{UMFI}_{\mathrm{cr}} / \mathrm{UMFI}_{\text {hir }}$ and $\mathrm{C}_{\mathrm{cr}} / \mathrm{C}_{\text {hir }}$ ratios under the operation at $5{ }^{\circ} \mathrm{C}$ was 0.78 and 0.58 , respectively; while the ratios at $20^{\circ} \mathrm{C}$ were significantly higher, 0.92 and 0.81 , respectively $(\mathrm{p}<0.05)$. Thus, chemical cleaning was not effective in removing irreversible fouling at $5{ }^{\circ} \mathrm{C}$ whereas at $20^{\circ} \mathrm{C}$, the foulants were effectively removed. The decrease in the cleaning efficiency at $5{ }^{\circ} \mathrm{C}$ is attributed to the high irreversible fouling (refer to Figure 5.5). At $35^{\circ} \mathrm{C}$, cleaning of the ceramic membrane was easier than $5{ }^{\circ} \mathrm{C}$ and $20^{\circ} \mathrm{C}$ conditions resulting in higher $\mathrm{UMFI}_{\mathrm{cr}} / \mathrm{UMFI}_{\text {hir }}$ and $\mathrm{C}_{\mathrm{cr}} / \mathrm{C}_{\text {hir }}$ ratios of 0.96 and 0.87 , respectively.

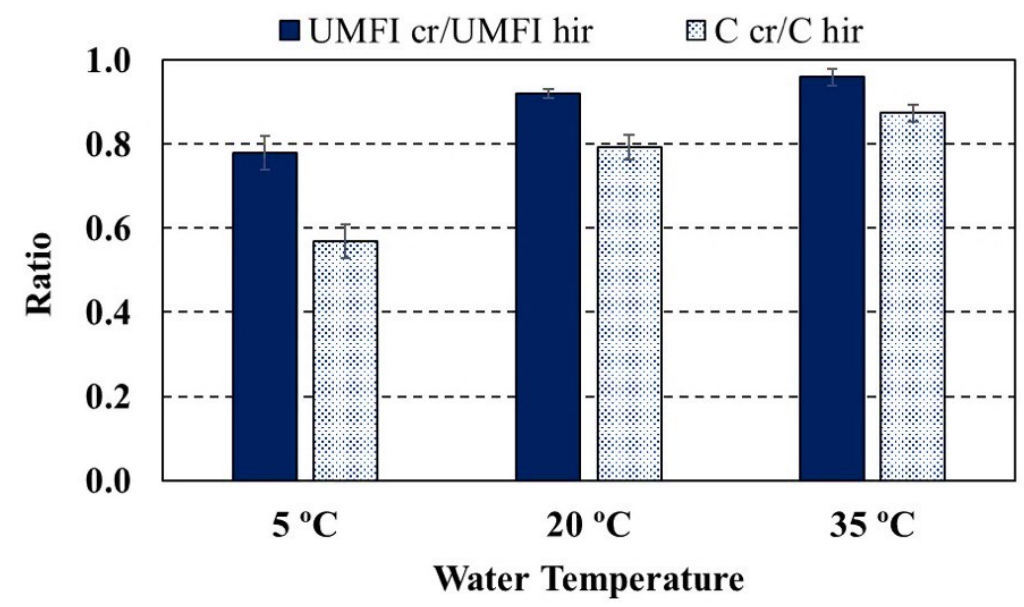

Figure 5.8 - Effect of water temperature on chemical cleaning efficiency* $\mathrm{UMFI}_{\mathrm{cr}} / \mathrm{UMFI}_{\text {hir }}$ : chemically reversible to hydraulically irreversible fouling ratios; $\mathrm{C}_{\mathrm{cr}} / \mathrm{C}_{\text {hir }}$ : chemically reversible to hydraulically irreversible carbon ratios.

*Note: The higher the UMFI $\mathrm{UL}_{\mathrm{r}} / \mathrm{UMFI}_{\text {hir }}$ ratio, the higher the cleaning efficiency; the higher the $\mathrm{C}_{\mathrm{rr}} / \mathrm{C}_{\text {hir }}$ ratio, the higher the removed carbon mass

FEEM plots of the $\mathrm{NaOCl}$ chemical wash water (Figure 5.9), were used to qualitatively assess the cleaning efficiency at different temperature. At $5{ }^{\circ} \mathrm{C}$ condition, higher intensities of humic acid (32.6 au) but much lower intensities for BSA/alginate (18.4 au). FEEM 
intensities at $20{ }^{\circ} \mathrm{C}$ for humic acid was 29.8 au which was approximately similar to that at $5{ }^{\circ} \mathrm{C}$, while much higher FEEM intensities for BSA/alginate $(38.1 \mathrm{au})$. These results indicate that the lower cleaning efficiency at $5{ }^{\circ} \mathrm{C}$ is attributed to the higher irreversible mass of BSA/alginate in the NOM mixture solution. Therefore, alteration of membrane chemical cleaning procedure at lower temperature is recommended to control chemically irreversible fouling and achieve higher permeability recovery. Chemical cleaning procedure was effective at both $20^{\circ} \mathrm{C}$ and $35^{\circ} \mathrm{C}(\mathrm{p}>0.05)$, refer to Figure 5.8, indicating that chemical cleaning procedure used at standard temperature $\left(20^{\circ} \mathrm{C}\right)$ should be sufficient at $35^{\circ} \mathrm{C}$ when needed.
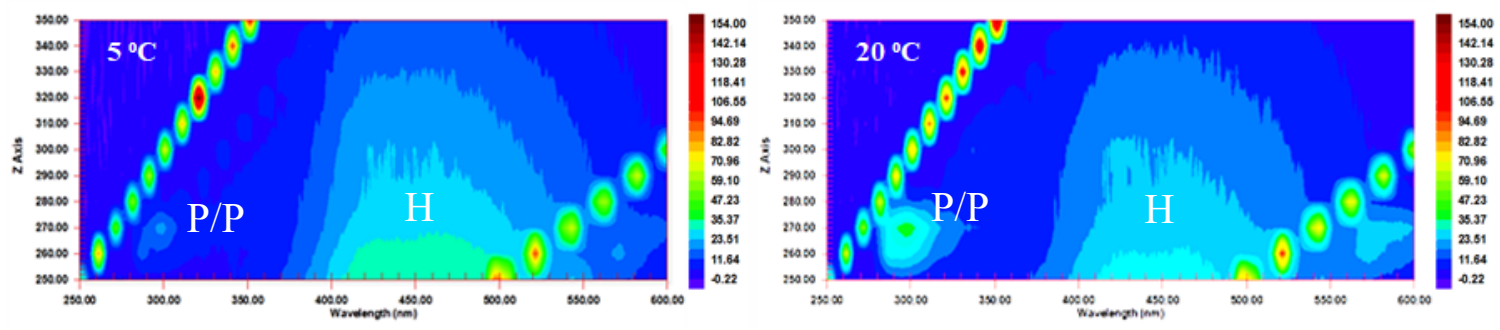

Figure 5.9- FEEM images of the ceramic UF $\mathrm{NaOCl}$ wash water of NOM mixture at 5 and $20^{\circ} \mathrm{C}$. P/P: (proteins, BSA/ polysaccharides); H: (humics)

\subsubsection{Suitability of the MFI-UF index for fouling prediction with ceramic membranes}

The MFI-UF and UMFI methods are used to evaluate membrane fouling, however, the different approaches in fouling assessment may suggest that direct comparison lack context. The MFI-UF provides an index value that indicates the fouling tendency of membrane feed whereas the UMFI provides information on the current fouling a membrane is subjected to (i.e. reversible vs. irreversible). Feed water having an MFI-UF $<3000 \mathrm{~s} / \mathrm{L}^{2}$ (equivalent to an $\mathrm{SDI}<3 \% / \mathrm{min}$ ), is considered acceptable for membrane feed (Boerlage 
et al., 2002). However, the actual capacity of the MFI-UF to be used effectively as a prediction tool with NOM fouling for ceramic membranes has yet to be evaluated in general and under changing water temperature conditions.

Figure 5.10 shows the fouling index values (Figure 5.10A), specific cake resistances (Figure 5.10B), and NOM retention (Figure 5.10C) at different water temperature conditions as predicted by the MFI-UF testing. The MFI-UF values and specific cake resistances were calculated following the procedure described in section 5.2.3.4. The results in Figure 5.10A show that the MFI-UF value of the NOM mixture decreased significantly $(\mathrm{p}<0.05)$ as the water temperature increased from 5 to $35^{\circ} \mathrm{C}$, indicating lower fouling potential at higher temperature. $\mathrm{NOM}$ in cold water temperatures (i.e. $5^{\circ} \mathrm{C}$ ) appears to have higher fouling potential (MFI-UF of $10122 \mathrm{~s} / \mathrm{L}^{2}$ ) compared to that at warmer temperatures (MFI-UF of $6850 \mathrm{~s} / \mathrm{L}^{2}$ and $3774 \mathrm{~s} / \mathrm{L}^{2}$ for $20{ }^{\circ} \mathrm{C}$ and $35{ }^{\circ} \mathrm{C}$, respectively). This could be a critical issue in assessing NOM fouling specifically in regions where there is a large seasonal variation in water temperature, as in North America and some parts of Europe. The decrease in water temperature caused changes to the cake layer that was formed and the amount of NOM retained by the ceramic UF as shown in Figures $5.10 \mathrm{~B}$ and $5.10 \mathrm{C}$. The specific cake resistance $\left(\alpha_{\mathrm{c}}\right)$ was found to increase with decreasing temperature from $20^{\circ} \mathrm{C}$ to $5^{\circ} \mathrm{C}$ (Figure 5.10B) along with an increase in NOM retention (Figure 5.10C). Therefore, the increase in the MFI-UF value at the lower temperature was related to the impact of temperature on BSA and alginate size (as shown in the FEEM images), resulting in high NOM retention by the UF membrane and an 
increase in specific cake resistance whereas at high temperature, NOM were passing through the UF membrane to the filtrate side, thus, lower fouling and MFI-UF value.

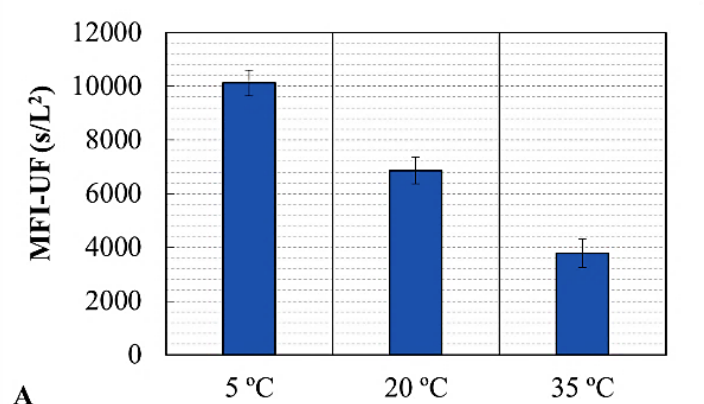

$\mathbf{A}$
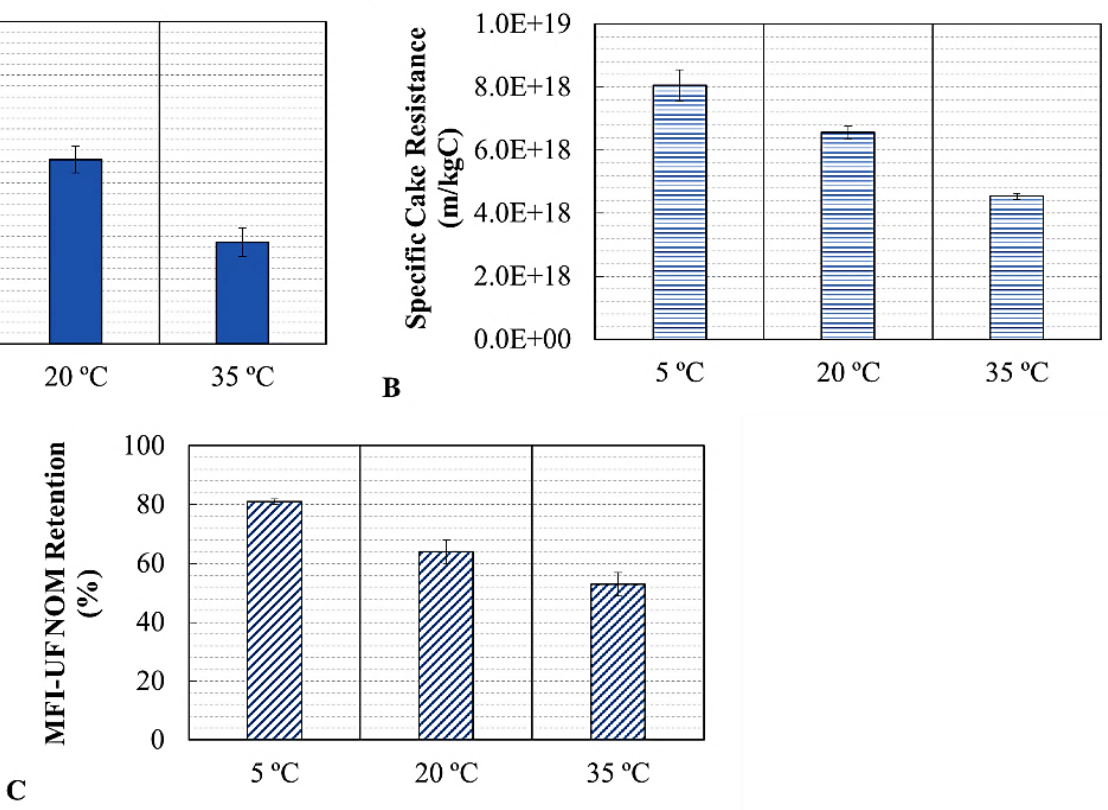

Figure 5.10 - (A) MFI-UF fouling index values at different temperature; (B) Changes in specific cake resistance with temperature based on the MFI-UF test; (C) NOM retention by the MFI-UF membrane at different temperature

Variation in water temperature demonstrated an impact on both the fouling potential of NOM (MFI-UF) and fouling mechanism (UMFI). Figure 5.11A shows that the MFI-UF fouling prediction trend is in agreement with the fouling tendency observed using the UMFI method. Moreover, Figures 5.11B and 5.11C show good correlations between specific cake resistance and NOM retention values obtained in the MFI-UF and ceramic UF systems, thus, the MFI-UF testing method demonstrates a useful fit for predicting NOM fouling and retention for ceramic membranes before relying on operating data (i.e. UMFI). 
Although there are differences in the numerical values of specific cake resistances and NOM retentions which are attributed to the differences in membrane materials and characteristics, the MFI-UF is simple and short filtration test that can be used as a rapid assessment of NOM fouling compared to the UMFI analysis which normally requires longterm filtration data. Thus, a ceramic membrane plant could use the MFI-UF in addition to the UMFI to assess potential changes in NOM fouling with temperature and make required alternations in membrane pretreatment and/or cleaning procedures.
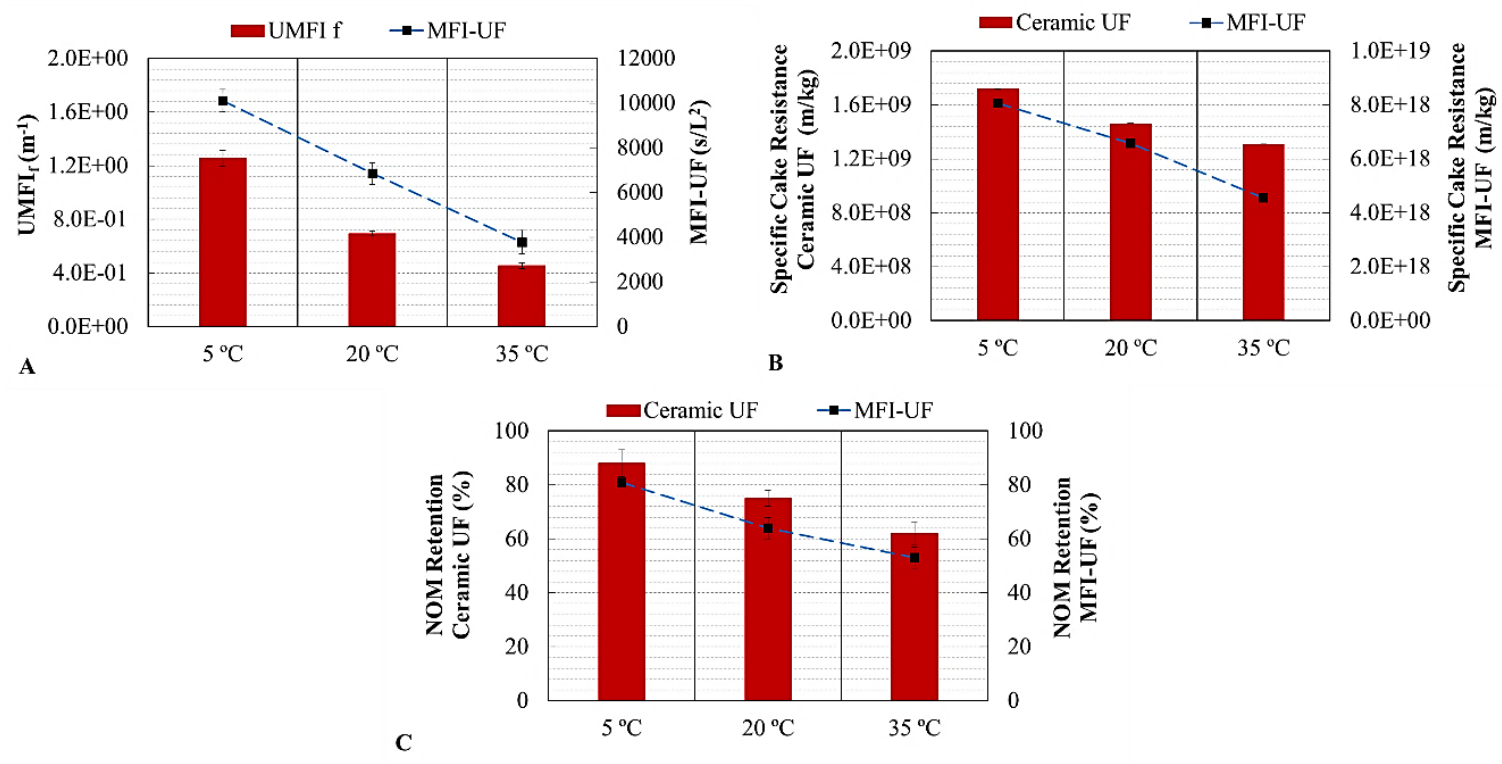

Figure 5.11 - (A) Relationship between $U_{M F I}$ and MFI-UF fouling indices; (B) Specific cake resistance with temperature; (C) NOM retention by MFI-UF and ceramic UF membranes

\subsection{Conclusions}

Water temperature is an important design parameter for membrane systems. This research investigated the impact of water temperature on NOM fouling and cleaning of a tubular 
ceramic UF membrane using the UMFI and MFI-UF fouling index methods. Key findings were:

- The effect of variation of feed water temperature on fouling of ceramic UF membranes was over and beyond simple viscosity changes of water. The $\mathrm{UMFI}_{\mathrm{f}}$ and NOM retention increased when the water temperature decreased from $35^{\circ} \mathrm{C}$ to $5{ }^{\circ} \mathrm{C}$ due to changes in NOM fouling behavior and specific fouling resistance. Thus, fouling analysis performed at standard temperature $\left(20^{\circ} \mathrm{C}\right)$ will underestimate the impact of cold water condition on ceramic membranes performance.

- The reversible and irreversible fouling ratio $\left(\mathrm{UMFI}_{\mathrm{hr}} / \mathrm{UMFI}_{\mathrm{hir}}\right)$ changed with temperature, thus, alteration of backwash procedure (i.e. increase or decrease in backwash pressure) with temperature is required to help control fouling and maintain membrane productivity.

- FEEM analysis demonstrated higher levels of protein removal with backwashing at 20 ${ }^{\circ} \mathrm{C}$ temperature versus $5{ }^{\circ} \mathrm{C}$; and thus FEEM is useful in determining specific fouling components that can be addressed under site specific NOM and temperature changes.

- Changes in NOM fouling characteristics with temperature as predicted by the MFI-UF method demonstrated useful fit with the UMFI values and NOM retention data obtained in the ceramic UF system. Thus, the utilization of the MFI-UF proved to be useful for assessing the potential changes in NOM fouling behavior with temperature for ceramic membrane systems. 


\subsection{Acknowledgements}

The authors would like to acknowledge the Saudi Arabia Ministry of Education (MOE) and Natural Sciences and Engineering Research Council of Canada (NSERC) for helping fund this research. We also wish to thank Geoff Seatter and Kerwin Lewis for their help with installation of the automated filtration system.

\subsection{References}

Alresheedi, M., Barbeau, B., Basu, O., 2019. Comparisons of NOM fouling and cleaning of ceramic and polymeric membranes during water treatment. Separation and Purification Technology 209, 452-460.

Alresheedi, M., Basu, O., 2018. Application of MFI-UF fouling index with NOM fouling under various operating conditions. Desalination and Water Treatment 133, 45-54.

ASTM International 2014. Standard test method for silt density index (SDI) of water. Designation: D4189 - 07.

Boerlage, S., Kennedy, M., Dickson, M., El-Hodali, D., Schippers, J., 2002. The modified fouling index using ultrafiltration membranes (MFI-UF): characterisation, filtration mechanisms and proposed reference membrane. Journal of Membrane Science 197, 1-21. 
Chang, H., Qu, F., Liy, B., Yu, H., Li, K., Shao, S., Li, G., Liang, H., 2015. Hydraulic irreversibility of ultrafiltration membrane fouling by humic acid: Effects of membrane properties and backwash water composition. Journal of Membrane Science 493, 723-733.

Chellam, S., Wendong, X., 2006. Blocking laws analysis of dead-end constant flux microfiltration of compressible cakes. Journal of Colloid Interface Science 301, 248-257.

Crittenden J., Trussell R., Hand D., Howe K., Tchobanoglous G., 2005. Water Treatment: Principles and Design, 2nd edition, Ed, Wiley, New Jersey, USA.

Cui, L., Goodwin, C., Gao, W., Liao, B., 2017. Effect of cold water temperature on membrane structure and properties. Journal of Membrane Science 540, 19-26.

Farahbakhsh, K., Smith, D., 2006. Membrane filtration for cold regions - impact of cold water on membrane integrity testing. Journal of Environmental Engineering and Science $5,69-75$.

Foley, G., 2006. A review of factors affecting filter cake properties in dead-end microfiltration of microbial suspensions. Journal of Membrane Science 274, 38-46.

Hamad, J., Ha, C., Kennedy, M., Amy, G., 2013. Application of ceramic membranes for seawater reverse osmosis (SWRO) pre-treatment. Desalination and Water Treatment 51, 4881-489. 
Hashino, M., Hirami, K., Ishigmi, T., Ohmukai, Y., Maruyama, T., Kubota, N., Matsuyama, H., 2011. Effect of kinds of membrane materials on membrane fouling with BSA. Journal of Membrane Science 384, 157-165.

Huang, H., Young, T.A., Jacangelo, J., 2008. Unified membrane fouling index for low pressure membrane filtration of natural waters: Principles and methodology, Environmental Science Technology 42 714-720.

Jeong, S., Vigneswaran, S., 2015. Practical use of standard pore blocking index as an indicator of biofouling potential in seawater desalination. Desalination 365, 8-14.

Jermann, D., Pronk, W., Boller, M., 2008. Mutual influences between natural organic matter and inorganic particles and their combined effect on ultrafiltration membrane fouling. Environmental Science and Technology 42, 9129-9136.

Jin, X., Jawor, A., Kim, S., Hoek, E., 2009. Effects of feed water temperature on separation performance and organic fouling of brackish water RO membranes. Desalination 239, 346359.

Katsoufidou, K., Yiantsios, S.G., Karabelas, A.J., 2007. Experimental study of ultrafiltration membrane fouling by sodium alginate and flux recovery by backwashing. Journal of Membrane Science 300, 137-146. 
Lee, H., Kim, S., Choi, J., Oh, H., Lee, W., 2013. Effects of water temperature on fouling and flux of ceramic membranes for wastewater reuse. Desalination and Water Treatment $51,5222-5230$.

Lee, S., Kim, J., 2014. Differential natural organic matter fouling of ceramic versus polymeric ultrafiltration membranes. Water Research 48, 43-51.

Ma, Z., Wen, X., Zhao, F., Xia, Y., Huang, X., Wait, D., Guan, J., 2013. Effect of temperature variation on membrane fouling and microbial community structure in membrane bioreactor. Bioresource Technology 133, 462-468.

Matilainen, A., Gjessing, E., Lathinen, T., Hed, L., Bhatnagar, A., Sillanpaa, M., 2011. An overview of the methods used in the characterisation of natural organic matter (NOM) in relation to drinking water treatment. Chemosphere 83, 1431-1442.

Roy, Y., Warsinger, D., Leinhard, J., 2017. Effect of temperature on ion transport in nanofiltration membranes: diffusion, convection and electro migration. Desalination, 420, $241-257$.

Samaei, S., Gato-Trinidad, S., Altaee, A., 2018. The application of pressure-driven ceramic membrane technology for the treatment of industrial wastewaters - A review. Separation and Purification Technology 200, 198-220. 
Schippers J., Verdouw J. 1980. the modified fouling index: a method of determining the fouling characteristics of water. Desalination 32, 137-148.

Stade, S., Kallioinen, M., Tuuva, T., Manttari, M., 2015. Compaction and its effect on retention of ultrafiltration membranes at different temperatures. Separation and Purification Technology 151, 211-217.

Steinhauer, T., Hanely, S., Bogendorfer, K., Kulozik, U., 2015. Temperature dependent membrane fouling during filtration of whey and whey proteins. Journal of Membrane Science 492, 364-370.

Taheri, A, Sim, L., Haur, C., Alkhondi, E., Fane, A., 2013. The fouling potential of colloidal silica and humic acid and their mixtures. Journal of Membrane Science 433, 112 120.

Van den Brink, P., Satpradit, O., Bentem, A., Zwijinenburg, A., Temmink, H., Loosdrecht, M., 2011. Effect of temperature shocks on membrane fouling in membrane bioreactors. Water Research 45, 4491-4500.

Vasanth, D., Pugazhenthi, G., Uppaluri, R., 2013. Cross-flow microfiltration of oil-inwater emulsions using low cost ceramic membranes. Desalination 320, 86-95. 
Villacorte, L., Ekowati, Y., Winters, H., Amy, G., Schippers, J., Kennedy, M., 2015. MF/UF rejection and fouling potential of algal organic matter from bloom-forming marine and freshwater algae. Desalination 367, 1-10.

Zhao, S., Zou, L., 2011. Effects of working temperature on separation performance, membrane scaling and cleaning in forward osmosis desalination. Desalination 278, $157-$ 164. 


\title{
Chapter 6
}

\section{Comparisons of NOM Fouling and Cleaning of Ceramic and Polymeric Membranes during Water Treatment}

\author{
Mohammad T. Alresheedi*, Benoit Barbeau**, Onita D. Basu* \\ * Department of Civil and Environmental Engineering, Carleton University, 1125 Colonel \\ By Drive, Ottawa, ON, K1S 5B6, Canada. \\ ${ }^{* *}$ Department of Civil, Geological and Mining Engineering, Polytechnique, C.P. 6079 \\ Succursale Centre-Ville, Montreal, QC, H3C 3A7, Canada. \\ Published: Journal of Separation and Purification Technology
}

\begin{abstract}
This research examines the effect of various NOM fractions on ceramic and polymeric UF membranes performance in terms of fouling and cleaning. Fouling experiments were performed using five model solutions, humic acid, protein as bovine serum albumin (BSA), alginate with and without calcium, and a combined NOM mixture. Two chemical agents were selected: an oxidant $(\mathrm{NaOCl})$ and caustic $(\mathrm{NaOH})$. Fouling and cleaning behavior were assessed using the resistance in series (RIS) model, membrane permeability, carbon mass balance, and fluorescence excitation and emission matrix (FEEM) analysis. The results demonstrated that NOM fouling order of the ceramic UF was similar to polymeric UF with the following trend: NOM mixture $\approx$ BSA $>$ alginate $\pm \mathrm{Ca}^{+2}>$ humic acid. However, the backwash efficiency was $1.5 \mathrm{x}-2 \mathrm{x}$ higher for the ceramic UF in comparison to the polymeric UF, indicating a much higher hydraulic reversibility for the ceramic UF. A carbon mass balance in complement with FEEM plots determined that NOM removal
\end{abstract}


by the ceramic UF was $\approx 10 \%$ higher than the polymeric UF. Chemical cleaning was found to be effective for both membrane types. Thus, it was not possible to conclude, that the ceramic membrane demonstrated an advantage for chemical cleaning under the conditions studied.

Keywords: Ceramic UF; Polymeric UF; NOM fouling; Chemical cleaning; Carbon balance

\subsection{Introduction}

Membrane processes are considered a reliable option for drinking water production. However, fouling by NOM, such as humic acids, proteins and polysaccharide-like substances, is one of the greatest challenges in membrane-based drinking water processes (Hashino et al., 2011; Katsoufidou et al., 2010). Fouling includes the short and long term loss of membrane permeability. The short term fouling is the reversible accumulation of NOM and particulate matter that can be removed by hydraulic backwash and/or chemical cleaning. Long term fouling is the slower loss of permeability due to the irreversible sorption of materials that resists hydraulic backwash and/or chemical cleaning. Irreversible fouling, in particular, is an inevitable phenomenon that results in the deterioration of membrane performance, chemical cleaning and membrane replacements, and hence increases operational cost of membrane systems.

Over the past years, research efforts have been focused on understanding fouling with a goal of minimizing it while improving filtration performance. In order to do so, the role of membrane material (i.e. type, hydrophobicity, etc.) has been highlighted as an important 
factor to minimize fouling (Hashino et al., 2011; Evans et al., 2008) with negatively charge/hydrophilic membranes being the preferred option for surface water treatment (Shao et al., 2011). In addition, the properties of NOM in water are considered an important factors that influences fouling. It was reported that hydrophobic NOM components, such as humic substances contributed to membrane fouling (Katsoufidou et al., 2005) while other studies found that polysaccharide-like substances, contributed to higher fouling than humic substances (Hashino et al., 2011; Katsoufidou et al., 2010). Synergy was also reported between different NOM fractions (Jamal et al., 2014; Katsoufidou et al., 2010) or NOM and inorganic matters (Jermann et al., 2008).

While the majority of past research was conducted using polymeric membranes, alternative ceramic membranes have received increasing attention in the drinking water industry. Ceramic membranes which are made of inorganic materials are superior to their polymeric counterpart in their mechanical and chemical characteristics allowing for higher fluxes and backwash pressures as well as aggressive cleaning approaches without risk of damaging membrane integrity (Lee and Kim, 2014; Pendergast and Hoek, 2011). These advantages can potentially increase the operational life expectancy of ceramic membranes and decrease the cleaning and replacement requirements. Moreover, ceramic membranes are expected to last 20 years compared to a typical 7-10 years with polymeric membranes leading to lower life-cycle costs than polymeric membranes (Wise, 2015). However, ceramic membranes currently impose higher initial capital costs compared to polymeric membranes which has thus far largely limited their use to industrial applications (e.g., food and beverage, oil and gas produced waters (Vasanth et al., 2013; Zhou et al., 2010). 
However, due to their operational advantages, the implementation of ceramic membranes for drinking water production is increasing and expected to grow over the next years.

Although some of the operational advantages of ceramic membranes are reported in the literature, few quantitative data exists about NOM fouling and the associated chemical cleaning protocols of ceramic membranes compared to their polymeric counterpart during surface water treatment. Oxidants and caustics are typically used to clean membranes fouled by organic compounds through oxidation, hydrolysis, and solubilization (Regula et al., 2014). However, the primary choice of appropriate cleaning agent for NOM is difficult as site specific needs should be considered (i.e. specific feedwater and membrane materials). Moreover, as irreversible fouling is known to be influenced by membrane and NOM properties (Hashino et al., 2011; Shao et al., 2011), NOM is expected to be less reactive with ceramic membranes due to the hydrophilic nature of the membrane (Lee and Kim, 2014; Zhou et al., 2010). Thus, research which examines clean in place (CIP) methodologies with different NOM fractions is required to increase fundamental understanding of cleaning regimes that control reversible and irreversible fouling for the two different membrane types. Therefore, the objectives of this research are to elucidate differences in fouling fractions (reversible vs irreversible) and cleaning efficiency of ceramic and polymeric UF membranes with various NOM types. The RIS model, membrane permeability, NOM mass balance and fluorescent excitation emission matrix (FEEM) analysis of permeate, backwash and chemical wash waters were used to investigate reversible and irreversible fouling and chemical cleaning performance using sodium hydroxide $(\mathrm{NaOH})$ and hypochlorite $(\mathrm{NaOCl})$. 


\subsection{Materials and Methods}

\subsubsection{Model foulants}

NOM model foulants used in this study were humic acids (Sigma Aldrich lot \#BCBK5107V) (2.5 mg C/L); a protein (bovine serum albumin, BSA, $2.5 \mathrm{mg} \mathrm{C/L}$ ); a polysaccharide (sodium alginate $2.5 \mathrm{mg} \mathrm{C} / \mathrm{L}$ ), average molecular weight between $30 \mathrm{kDa}$ - $100 \mathrm{kDa}$ (Katsoufidou et al., 2010); sodium alginate with $\mathrm{Ca}^{+2}$ addition $(75 \mathrm{mg} / \mathrm{L}$ as $\left.\mathrm{CaCO}_{3}\right)$ using calcium sulfate $\left(\mathrm{CaSO}_{4}\right)$; and a mixture of the three NOM models, 0.83 $\mathrm{mgC} / \mathrm{L} /$ each model (2.5 $\mathrm{mg} \mathrm{C} / \mathrm{L}$ as final concentration). The humic acid model (Sigma Aldrich) has been previously used in numerous membrane fouling investigations because of its fouling propensity (to accelerate fouling) and known characteristics. The test with alginate/ $\mathrm{Ca}^{+2}$ was included in the experimental design due to the high fouling propensity of this combination (Jamal et al., 2014). All model substances were purchased from Sigma Aldrich. Model solutions were prepared using pure deionized (DI) water and were mixed using a magnetic stirrer one-day prior to experiments ensuring that materials were dissolved completely. In addition, turbidity ( $5 \mathrm{mg} / \mathrm{L}$ of kaolin clay, equivalent to $5 \mathrm{NTU}$ ), and alkalinity $\left(75 \mathrm{mg} / \mathrm{L}\right.$ as $\left.\mathrm{CaCO}_{3}\right)$ using sodium bicarbonate $\left(\mathrm{NaHCO}_{3}\right)$ were added. The $\mathrm{pH}$ of the feed was maintained around 7.5. NOM molecular weight distributions of synthetic solutions and zeta potential (as shown in Table 6.1 were measured using a UF fractionation method described by Kitis et al., (2002) and a Malvern Nano-sizer. 
Table 6.1 - Molecular weight fractionation and zeta potential of feed solutions

\begin{tabular}{cccccc}
\hline \multicolumn{5}{c}{ Model feed NOM solution } \\
\hline Molecular Weight & $\begin{array}{c}\text { Humic } \\
\text { acids }\end{array}$ & BSA & $\begin{array}{c}\text { Sodium } \\
\text { alginate } \\
-\mathrm{Ca}^{+2}\end{array}$ & $\begin{array}{c}\text { Sodium } \\
\text { alginate } \\
+\mathrm{Ca}^{+2}\end{array}$ & Mixture \\
\hline$>100 \mathrm{kDa}$ & $32 \%$ & $25 \%$ & $28 \%$ & $36 \%$ & $38 \%$ \\
$30 \mathrm{kDa}-100 \mathrm{kDa}$ & $38 \%$ & $61 \%$ & $30 \%$ & $48 \%$ & $41 \%$ \\
$10 \mathrm{kDa}-30 \mathrm{kDa}$ & $18 \%$ & $5 \%$ & $22 \%$ & $6 \%$ & $10 \%$ \\
$5 \mathrm{kDa}-10 \mathrm{kDa}$ & $9 \%$ & $5 \%$ & $10 \%$ & $3 \%$ & $5 \%$ \\
$1 \mathrm{kDa}-5 \mathrm{kDa}$ & $2 \%$ & $2 \%$ & $6 \%$ & $4 \%$ & $1 \%$ \\
$<1 \mathrm{kDa}$ & $1 \%$ & $2 \%$ & $4 \%$ & $2 \%$ & $3 \%$ \\
\hline Zeta Potential $(\zeta)$ & $-27 \pm 5$ & $-23 \pm 4$ & $-36 \pm 7$ & $-20 \pm 5$ & $-22 \pm 7$ \\
$\mathrm{mV}$ & & & & &
\end{tabular}

\subsubsection{Fouling experiments}

Assays were conducted on an automated filtration system (Figure 6.1) consisting of a digital gear pump (Cole Parmer: Drive no. 75211-30, Head no. 07003-04), pressure transducer (Omega: model no. PX409-100G5V), flow meter (Cole Parmer: model no. 32703-52), solenoid valves (Macmaster: model no. 4711K731), and pressure vessel (Cole Parmer: model no. 29902-90) and nitrogen gas for backwash. Labview program code (National Instruments, NI, USA) was created to operate the system automatically such as controlling the feed pump to have a constant flux, and automatically switches from filtration cycles to backwash cycles while recording flux and pressure data. 


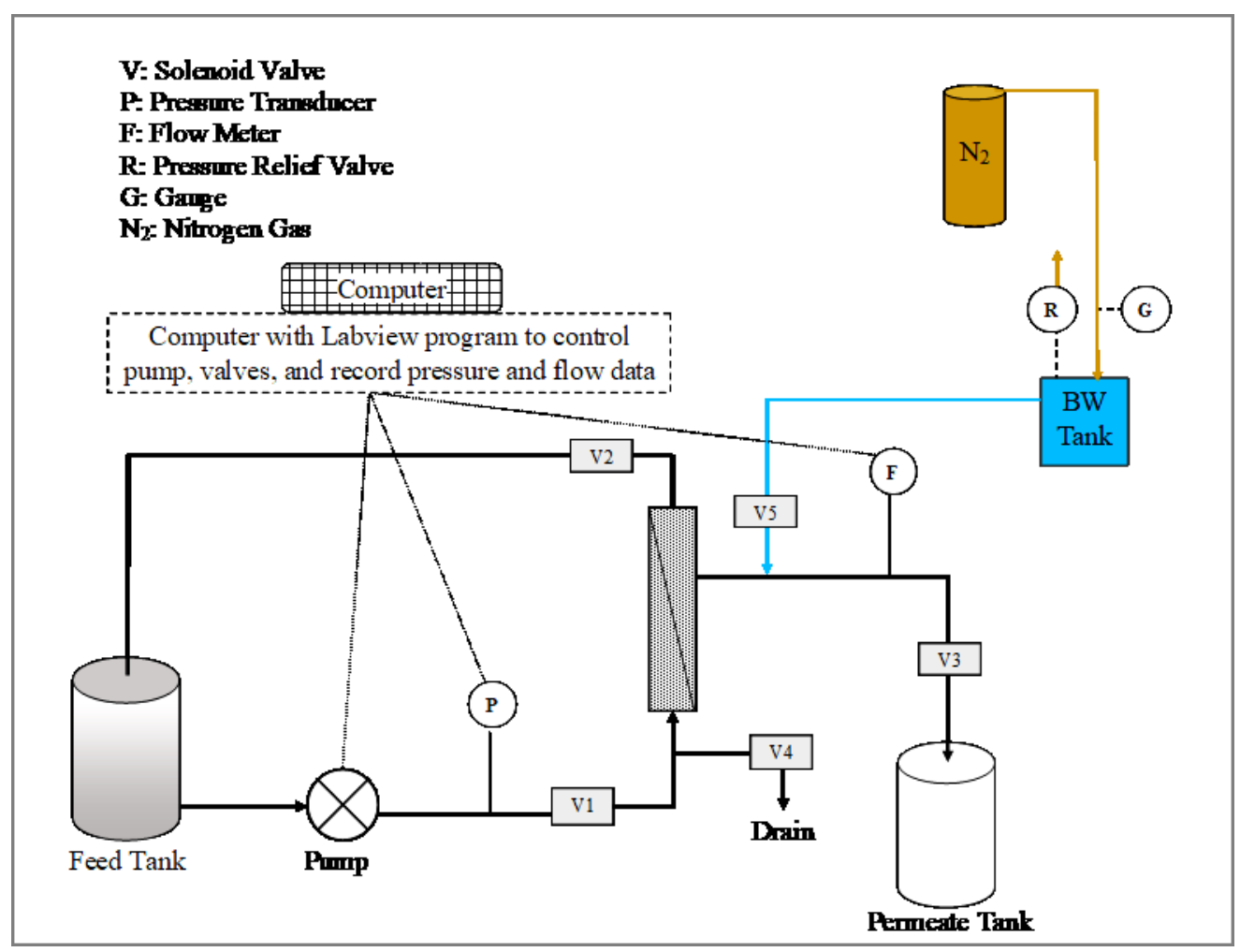

Figure 6.1- Automated membrane system

A ceramic UF $\left(\right.$ Ceramem $^{\mathrm{TM}}$, Veolia) and polymeric UF (X-Flow ${ }^{\mathrm{TM}}$, Pentair) were used during this research and their characteristics are presented in Table 6.2. It should be noted that the characteristics of the two membranes (i.e. dimensions, area, and filtration configuration) were approximately similar to facilitate direct comparisons. 


\subsubsection{Chemical cleaning experiments}

The goal of the chemical cleaning experiments was to provide direct comparisons of ceramic and polymeric membranes under equivalent cleaning protocols. CIP cleaning was performed using two common organic fouling cleaners: an oxidant, $\mathrm{NaOCl}$ or caustic

cleaning, $\mathrm{NaOH}$. Each cleaning was performed for 4 hours at a cleaning temperature of $35^{\circ} \mathrm{C}$ and $\mathrm{pH}$ of 11.0 for both cleaning solutions. Chemicals were recirculated at crossflow velocity of $0.1 \mathrm{~m} / \mathrm{s}$ for 1 hour followed by 3 hours of soaking. For the $\mathrm{NaOCl}$ chemical wash, the free chlorine concentration was $500 \mathrm{mg} \mathrm{Cl}_{2} / \mathrm{L}$.

\subsubsection{Analysis}

6.2.4.1 Fouling resistances and specific flux recovery

To quantify membrane fouling during membrane filtration, total fouling resistance $\left(\mathrm{R}_{\mathrm{f}}\right)$, hydraulically reversible fouling resistance $\left(\mathrm{R}_{\mathrm{hr}}\right)$, hydraulically irreversible fouling resistance $\left(R_{\text {hir }}\right)$, chemically reversible fouling resistance $\left(R_{c r}\right)$, and chemically irreversible fouling resistance $\left(\mathrm{R}_{\text {cir }}\right)$ were estimated using the slope of the normalized pressure $\left(\mathrm{P} / \mathrm{P}_{0}\right)$ and specific water volume (V/A) graph, (Figure 6.2) (Nguyen et al., 2011). The importance of each fouling resistance with respect to membrane operation is as follows; the greater the $\mathrm{R}_{\mathrm{hr}} / \mathrm{R}_{\mathrm{f}}$ ratio, the more effective the hydraulic backwashes, possibly due to greater cake formation, the greater the $\mathrm{R}_{\text {hir }} / \mathrm{R}_{\mathrm{f}}$ ratio, the greater the NOM adsorption and pore blockage and thus greater the need for chemical cleans as hydraulic backwashes are ineffective. The $\mathrm{R}_{\text {hir }} / \mathrm{R}_{\mathrm{f}}$ ratio can be categorized into two ratios: the chemically reversible fouling ratio $\left(\mathrm{R}_{\text {cr }} / \mathrm{R}_{\text {hir }}\right)$ and the chemically irreversible fouling $\left(\mathrm{R}_{\text {cir }} / \mathrm{R}_{\text {hir }}\right) . \mathrm{R}_{\mathrm{cr}} / \mathrm{R}_{\text {hir }}$ quantifies the fouling 
ratio removed by a chemical clean while $\mathrm{R}_{\text {cir }} / \mathrm{R}_{\text {hir }}$ quantifies the fouling ratio that remains after a chemical clean.

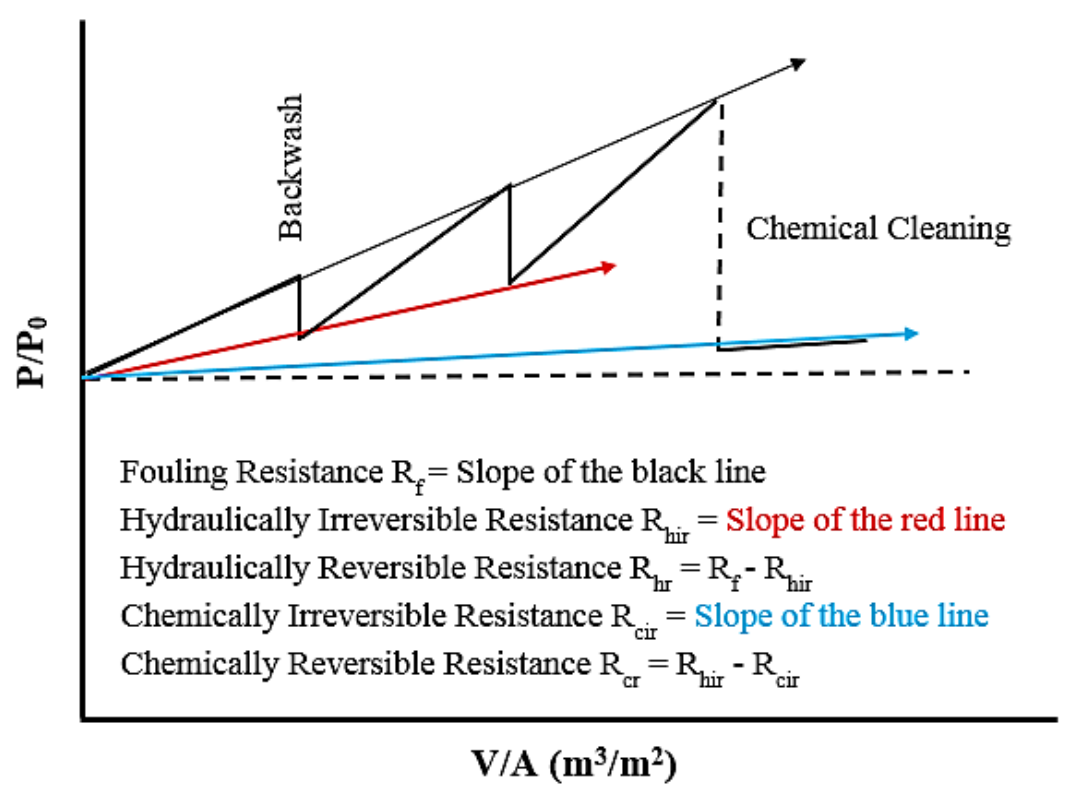

Figure 6.2- Resistances estimation using the slopes fouling graphs

The efficiency of chemical cleaning was assessed by comparing the chemically fouling resistance removal $\left(\mathrm{R}_{\mathrm{cr}}\right)$ and specific flux recovery (SFR) using Equations 6.2 and 6.3.

$$
\begin{aligned}
& \% \mathrm{R}_{\mathrm{Cr}}=\frac{\mathrm{R}_{\mathrm{cr}}(\text { removed by each cleaning step })}{\mathrm{R}_{\text {hir }}(\text { hydraulically irreversible fouling resistance })} \times 100 \\
& \% \mathrm{SFR}=\frac{\mathbf{J s p} \text { (after cleaning })}{\mathbf{J s p}_{\text {initial }}} \times 100
\end{aligned}
$$

\subsubsection{Carbon mass balance}

A carbon mass balance was performed to measure how much foulants were removed after each cleaning step and compare it to the fouling resistance removal data. This was done by 
collecting samples from feed, permeate, backwash and chemical wash water and performing TOC analysis (using a Sievers ${ }^{\mathrm{TM}}$ analyzer, GE Water) then converting the values to \% mass for calculations (Equation 6.4).

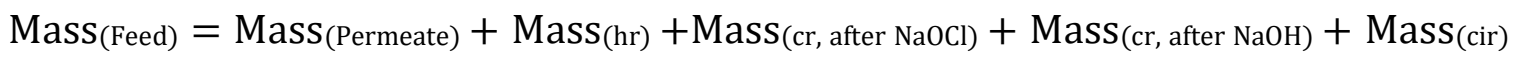
Equation (6.4)

Where: hr (hydraulically reversible carbon); cr (chemically reversible carbon); cir (chemically irreversible carbon)

\subsubsection{Fluorescence excitation and emission matrix (FEEM)}

FEEM analysis was performed on water samples of the mixture solution for more insights into cleaning of different NOM compositions. Samples were collected from permeate, backwash and chemical wash waters. A spectrofluorophotometer, RF-5301 PC (Shimadzu), was used for the FEEM analysis. The excitation wavelength was set to 340 $\mathrm{nm}$ and the emission wavelength ranged from $300 \mathrm{~nm}$ to $600 \mathrm{~nm}$. High sensitivity/slow scanning speed was used. Before analysis, a FEEM spectra of a blank was determined, then, the FEEM spectra of the analyzed sample was subtracted by the blank spectra. Python code, using Python xy, was used to plot the fluorescence data. 


\subsection{Results and Discussion}

\subsubsection{Ceramic versus polymeric UF performance comparison}

\subsubsection{NOM fouling and removal by ceramic and polymeric UF membranes}

Figures $6.3 \mathrm{~A}$ and $6.3 \mathrm{~B}$ show the ceramic and polymeric UF fouling from different NOM solutions during each filtration cycle with respect to normalized pressure. NOM mixture exhibited the most severe fouling among the five model solutions. The presence of $\mathrm{Ca}^{+2}$ during the filtration of sodium alginate solution demonstrated a noticeable adverse effect on fouling. The zeta potential of the alginate solution was reduced from $-36.7 \mathrm{mV}$ to -20.5 $\mathrm{mV}$ when $\mathrm{Ca}^{+2}$ was added. The zeta potential reduction caused by $\mathrm{Ca}^{+2}$ addition could be attributed to the aggregation of $\mathrm{Ca}^{+2}$ and alginate molecules, thus, reduction of the electrostatic repulsion forces between alginate and membrane surface, thus increasing fouling rate (Jermann et al., 2008). The effect of $\mathrm{Ca}^{+2}$ addition on alginate fouling analogous to other reported results (Zularisam et al., 2011; Jermann et al., 2008) using polymeric membranes who found that flux decline by alginate and $\mathrm{Ca}^{+2}$ mixture increased significantly during filtration and resulted in alginate/ $\mathrm{Ca}^{+2}$ gel of a high specific resistance. 


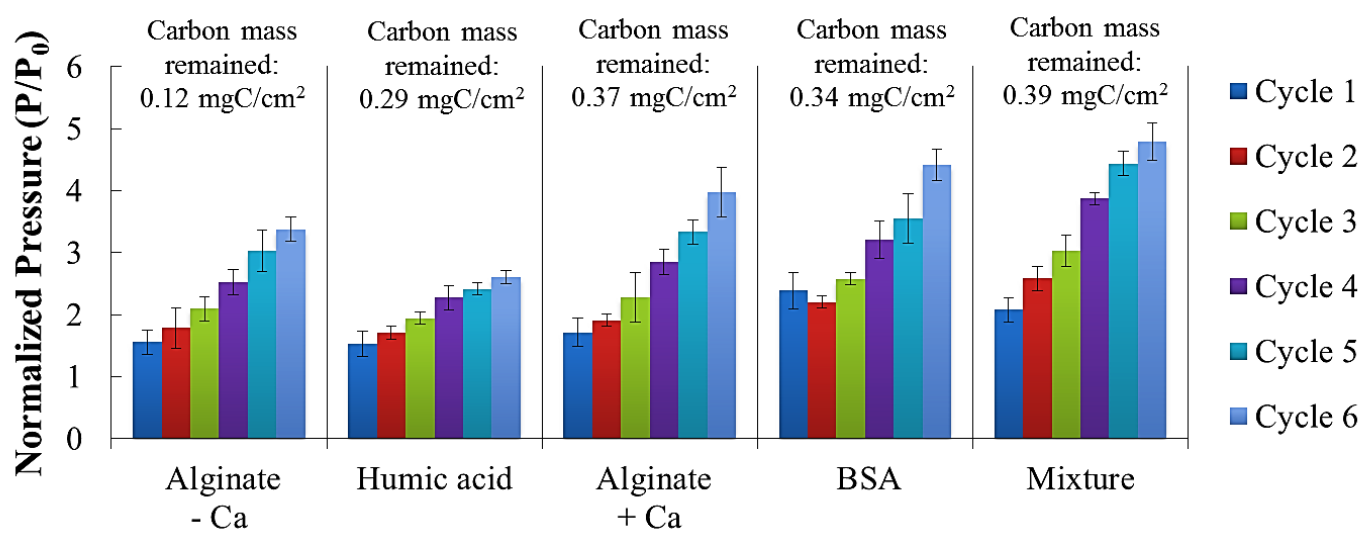

(A) Ceramic UF

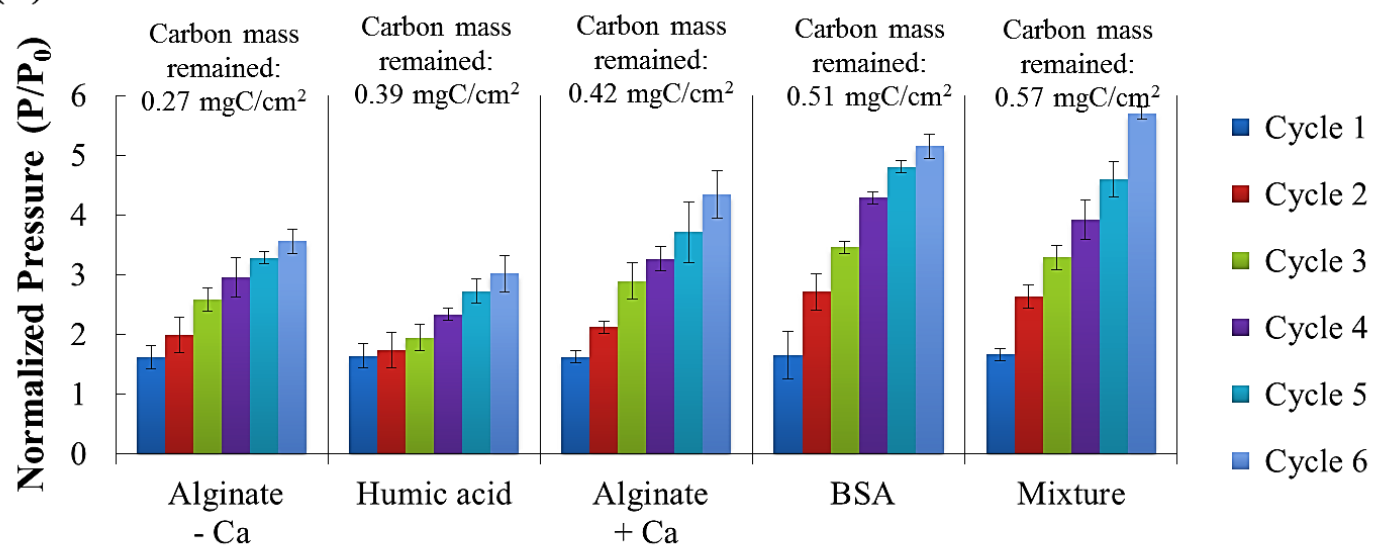

(B) Polymeric UF

Figure 6.3 - (A) Ceramic UF fouling by different NOM solutions (Flux: 100 LMH, BW: every 4 hours for 20s at 30 psi); (B) Polymeric UF fouling by different NOM solutions (Flux: $100 \mathrm{LMH}, \mathrm{BW}$ : every 4 hours for 20s at $30 \mathrm{psi}$ ).

Fouling of different NOM solutions of the ceramic UF was similar to the polymeric UF in terms of order; however, higher fouling was found with the polymeric UF. As backwash conditions were identical, we suggest that the difference in fouling can be attributed to the difference in material characteristics of both membranes. Previous studies suggested that membrane fouling was governed by membrane characteristics such as hydrophobicity and configuration. Lee et al. (2005) found that UF membrane with smoother surface had less fouling, while MF membrane with rough surface had severe flux decline due to deposition 
of fouling materials in the valleys of the membrane surface. Polymeric membranes with lower surface porosity resulted in lower humic acid rejection and significant flux decline (Fu et al., 2008). Membranes with hydrophobic behavior was found to be more susceptible to membrane fouling than hydrophilic (Gray et al., 2007). In this study, the pore size of the two membranes is similar; however, the polymeric UF surface is hydrophobic, promoting a stronger interaction between foulants and membrane surface. In contrast, the ceramic UF surface is hydrophilic and can easily form bonds with water molecules which would reduce fouling. Another important factor is the surface charge of both membranes. SiC (ceramic) and PES (polymeric) membranes have reported point-of-zero charges of 2.5-3.5 and 4.5, respectively (Hofs et al., 2011). At our neutral $\mathrm{pH}$ of operation, both membranes were expected to exhibit negative charges and therefore repulsions were expected. However, due to the lower point of zero charge, higher electrostatic repulsion was therefore favoring the ceramic membrane. In summary, although the fouling order was similar, the ceramic UF demonstrated a better performance than polymeric UF in terms of lowering fouling rate which could either be due to more effective backwash or lower NOM retention by the ceramic membranes.

In order to validate the difference in NOM retention achieved by both membranes, NOM removals based on influent/effluent TOC concentrations are presented in Table 6.3. Removal results indicate that the highest TOC removal was for BSA and sodium alginate $\left(+\mathrm{Ca}^{+2}\right)$ whereas lower removal for humic acids, sodium alginate $\left(-\mathrm{Ca}^{+2}\right)$ and the mixture solutions. However, although the removals for humic acid, sodium alginate $\left(-\mathrm{Ca}^{+2}\right)$ and the mixture solutions were approximately similar, it is unlikely that the removal mechanism 
was the same since the mixture resulted in high levels of hydraulically irreversible fouling whereas sodium alginate $\left(-\mathrm{Ca}^{+2}\right)$ fouling was mostly reversible. The increase in alginate removal with $\mathrm{Ca}^{+2}$ addition could be attributed to the aggregation effect, thus increasing particle size and removal.

Table 6.3- NOM removals by the ceramic and polymeric UF (95\% confidence interval)

\begin{tabular}{ccc}
\hline NOM Solution & Ceramic UF & Polymeric UF \\
\hline Humic Acid & $67 \% \pm 2 \%$ & $62 \% \pm 3 \%$ \\
BSA & $84 \% \pm 4 \%$ & $77 \% \pm 4 \%$ \\
Alginate $+\mathrm{Ca}^{+2}$ & $80 \% \pm 4 \%$ & $76 \% \pm 2 \%$ \\
Alginate $-\mathrm{Ca}^{+2}$ & $66 \% \pm 3 \%$ & $71 \% \pm 6 \%$ \\
Mixture & $73 \% \pm 2 \%$ & $64 \% \pm 3 \%$ \\
\hline
\end{tabular}

NOM removal by the ceramic and polymeric UF membranes were statistically analyzed using SPSS 22 by performing a Completely Randomized Block Design (CRBD) analysis. In $\mathrm{CRBD}$, the experimental units are grouped into blocks according to known or suspected variation which is isolated by the blocks (Montgomery et al., 2013). The NOM solution is the known source of variation in the removal data and therefore, it was used as blocks while comparing the removal differences between the two membrane materials. CRBD analysis results (Appendix D) indicate that NOM removal was significantly influenced by membrane materials $(\mathrm{p}=0.008)$, which could be attributed to the differences in the membrane structure and geometry. In other words, the overall higher level of removal of NOM between the ceramic and polymeric membranes can be attributed to the different membrane properties. 


\subsubsection{Hydraulic BW efficacy for ceramic and polymeric UF membranes}

Table 6.4 presents the $R_{h r}$ (hydraulically reversible resistance) and $R_{h i r}$ (hydraulically irreversible resistance) values estimated using the slope of fouling graphs. $R_{\text {hir }}$ was the highest for the NOM mixture, 3.97E-3 and 6.76E-3, for the ceramic UF and polymeric UF respectively. Interestingly, the fouling resistances caused by the BSA solution alone was very close to that by mixture solution. This demonstrates the effect of BSA on membrane fouling. The $\mathrm{R}_{\mathrm{hr}}$ values for the ceramic UF were always higher than that for the polymeric UF, which indicates that under identical filtration conditions, the interaction between NOM and ceramic membrane surface was weak, thus, high efficiency of backwashing with the ceramic UF than the polymeric UF. As a result, lower $R_{\text {hir }}$ values were found with the ceramic UF compared to polymeric UF.

Table 6.4- $\mathrm{R}_{\mathrm{hr}}$ and $\mathrm{R}_{\text {hir }}$ slope values of different feed NOM solutions

\begin{tabular}{c|cc|cc}
\hline & \multicolumn{2}{c}{ Ceramic UF } & \multicolumn{2}{c}{ Polymeric UF } \\
\hline & $\begin{array}{c}\mathrm{R}_{\mathrm{hr}}\left(\mathrm{\times} 10^{-3}\right) \\
\mathrm{m}^{-1}\end{array}$ & $\begin{array}{c}\mathrm{R}_{\mathrm{hir}}\left(\mathrm{\times} 10^{-3}\right) \\
\mathrm{m}^{-1}\end{array}$ & $\begin{array}{c}\mathrm{R}_{\mathrm{hr}}\left(\times 10^{-3}\right) \\
\mathrm{m}^{-1}\end{array}$ & $\begin{array}{c}\mathrm{R}_{\mathrm{hir}}\left(\mathrm{\times 10} 0^{-3}\right) \\
\mathrm{m}^{-1}\end{array}$ \\
\hline Alginate $-\mathrm{Ca}^{+2}$ & 5.60 & 1.36 & 4.30 & 2.78 \\
Humic acid & 4.20 & 1.05 & 2.90 & 2.46 \\
Alginate $+\mathrm{Ca}^{+2}$ & 4.10 & 2.89 & 1.70 & 5.60 \\
BSA & 3.31 & 3.72 & 1.80 & 6.55 \\
Mixture & 3.50 & 3.97 & 1.65 & 6.76 \\
\hline
\end{tabular}

Figure 6.4 illustrates the backwash efficiency (represented by the $R_{h r} R_{f}$ ratios) for the ceramic and polymeric UF. Overall, hydraulically reversible fouling resistance contributed between $\approx 50-80 \%(20-50 \%$ irreversible fouling) and $20-60 \%$ (40-80\% irreversible 
fouling) for the ceramic and polymeric UF respectively. Importantly, under an equivalent backwash procedure, the $R_{h r} R_{f}$ ratios were $1.5 x-2 x$ higher for the ceramic UF in comparison to the polymeric UF. Thus, the foulants were effectively removed more easily from the ceramic membrane through a simple backwash compared to the polymeric membrane. The superior backwash efficiency of the ceramic UF can potentially result in higher savings in ceramic membrane operational costs through the reduction of hydraulically irreversible fouling, and chemical cleaning frequency. Fouling of the alginate solution showed different trends with and without $\mathrm{Ca}^{+2}$ addition. Fouling was mostly irreversible during the filtration of the alginate $\left(+\mathrm{Ca}^{+2}\right)$ solution, whereas it was reversible for the alginate $\left(-\mathrm{Ca}^{+2}\right)$ solution. This demonstrates the adverse impact of $\mathrm{Ca}^{+2}$ ions on alginate irreversibility which explain the difference in fouling behavior observed during filtration (Figure 6.3). Therefore, it is necessary to be aware of the interactions of water quality characteristics when utilizing membranes for NOM treatment.

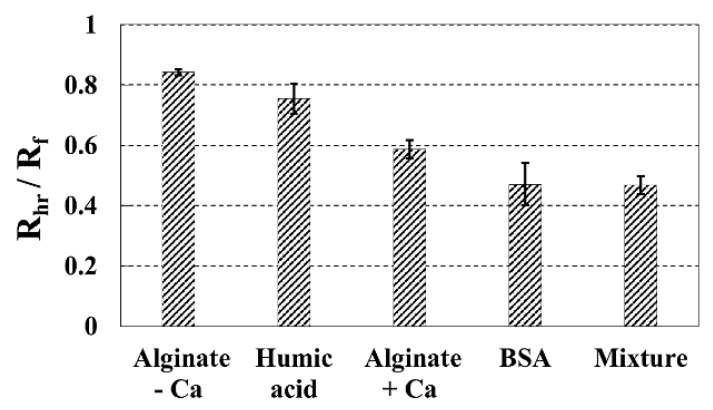

(A) Ceramic UF

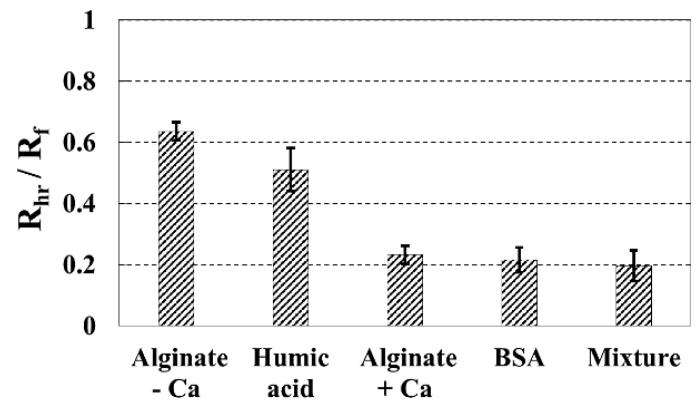

(B) Polymeric UF

Figure 6.4 - Backwash efficiency ( $\mathrm{R}_{\mathrm{hr}} \mathrm{R}_{\mathrm{f}}$ ratios). (A) Ceramic UF; (B) Polymeric UF. Higher ratios indicate higher BW efficiency and lower irreversibility. 


\subsubsection{Fouling analysis based on a carbon mass balance}

Figure 6.5 shows the carbon mass balance found in the backwash water (hydraulically reversible), remained on the membrane (hydraulically irreversible) and in the permeate water. The results illustrate that for all model solutions, except the alginate $\left(-\mathrm{Ca}^{+2}\right)$, more than $50 \%$ of NOM was hydraulically irreversible. This demonstrates that NOM particles strongly adsorbed to the membrane surface or pores causing higher fouling irreversibility. During the filtration of alginate $\left(-\mathrm{Ca}^{+2}\right), \approx 30-52 \%$ of NOM is found in the backwash water compared to $15-28 \%$ for alginate $\left(+\mathrm{Ca}^{+2}\right)$, which demonstrate the effect of $\mathrm{Ca}^{+2}$ on fouling reversibility/irreversibility of alginate. As observed earlier, the ceramic UF showed better removals of the hydraulically reversible carbon during backwashing demonstrating that under the same backwash procedure, the ceramic UF cleaning was more effective than the polymeric UF, highlighting an operational benefit of ceramic membranes over polymeric membranes. The carbon mass found in the permeate water was low: between $6-12 \%$ for the ceramic UF and 11-18\% for the polymeric UF which indicate the higher NOM rejection and better permeate quality for the ceramic UF compared to the polymeric UF. The results of the carbon mass balance correlate well with the fouling resistance in series analysis and NOM removal data, and therefore, carbon mass balance could be a useful approach for fouling quantification in pilot and full-scale settings compared to RIS, which requires specific operator skill and experience and can also be time consuming and difficult to conduct on full-scale data. 


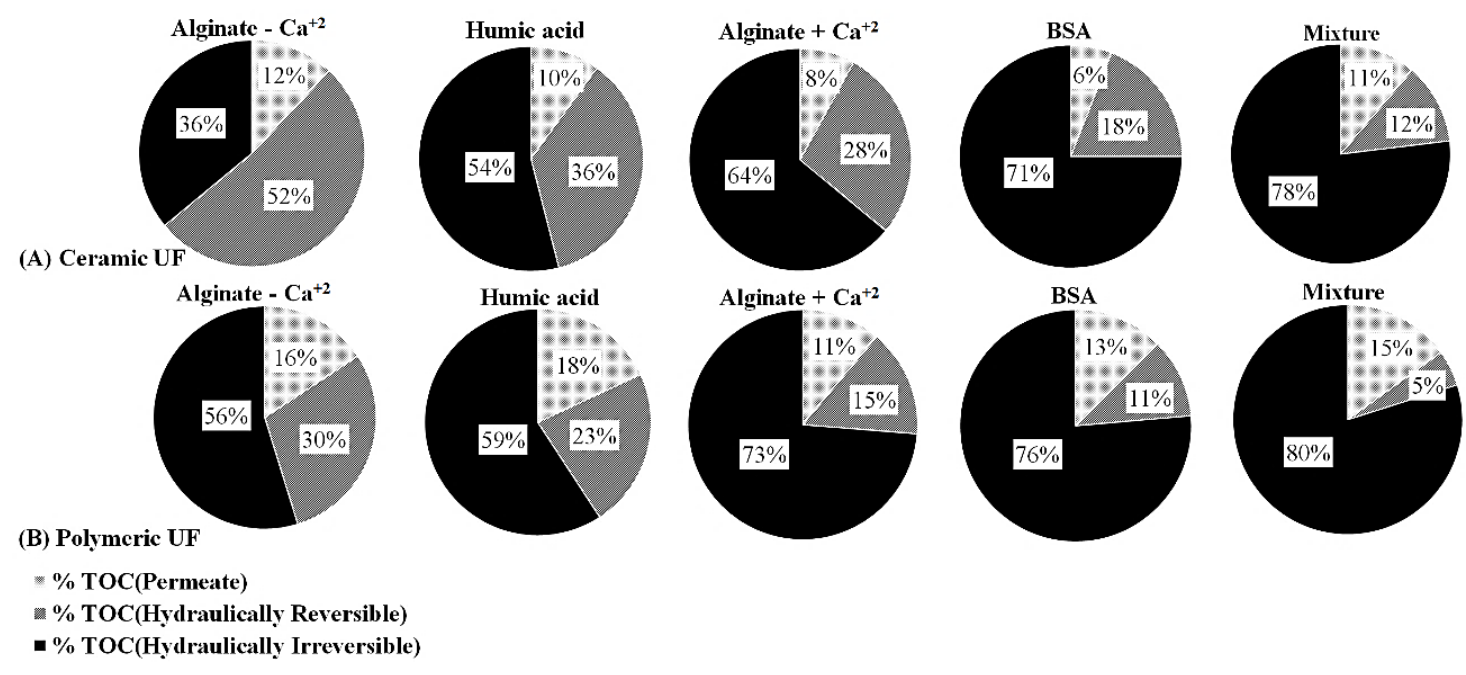

Figure 6.5 - Mass balance of carbon (expressed as \% of the feed) found as hydraulically reversible (in the BW water), hydraulically irreversible (left on the membrane) or in the permeate water.

\subsubsection{FEEM analysis of permeate and backwash waters}

FEEM analysis was used to visualize the foulants in the ceramic and polymeric UF permeate and backwash water. Figure 6.6 shows fluorescence plots for the NOM mixture solution. The fluorescence peaks for humic-like substances are reported from 300-390 nm (range of excitation) and between 400-500 nm (range of emission). The fluorescence peaks for protein-like substances are around 230-280 nm (range of excitation) and between 310$360 \mathrm{~nm}$ (range of emission) (Matilainen et al., 2011). Backwash water samples from both membranes show high fluorescence intensity $(\approx 900)$ in the region between Ex/Em 250$300 / 400-450 \mathrm{~nm}$ which indicates the presence of humic acids. BSA is also found in backwash water, however in less intensity than humic acids $(\approx 300)$. Higher concentrations are found in the ceramic UF backwash water indicating better backwash efficiency than for the polymeric membrane. In addition, the ceramic UF permeate shows low FEEM intensities $(\approx 250)$ than the polymeric UF permeate $(\approx 400)$. These results are coherent 
with the resistance-in-series and carbon mass analysis. Hydraulic BW are more effective on the ceramic membranes which leads to lower hydraulically irreversible fouling.
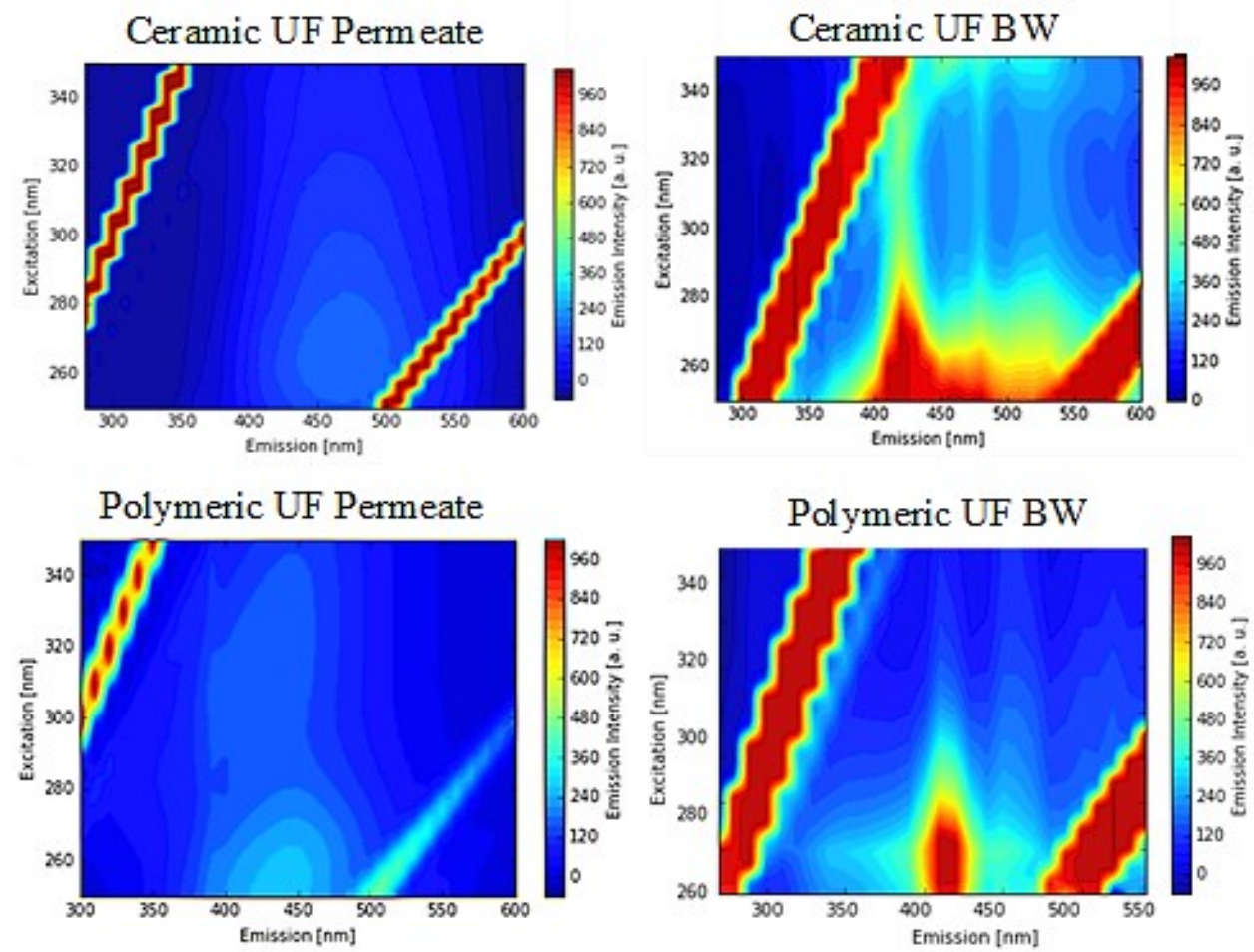

Figure 6.6 - FEEM plots of NOM mixture (permeate and backwash water)

\subsubsection{Ceramic versus polymeric UF cleaning under equivalent cleaning protocols}

\subsubsection{Fouling resistance removal and specific flux recovery}

After fouling experiments, both membranes were cleaned at $\mathrm{pH} 11.0$ using $\mathrm{NaOCl}$ (500 $\mathrm{mg} \mathrm{Cl}_{2} / \mathrm{L}$ ) or $\mathrm{NaOH}$. Figures $6.7 \mathrm{~A}-\mathrm{B}$ show the chemical cleaning efficiency of different NOM foulants using the chemically reversible resistance $\left(\mathrm{R}_{\mathrm{cr}}\right)$ for the two cleaning conditions. For the ceramic UF, $\% \mathrm{R}_{\mathrm{cr}}$ after $\mathrm{NaOCl}$ cleaning was around $80 \%, 60 \%, 74 \%$, $62 \%$ and $58 \%(\%$ SFR range was $65-85 \%)$ whereas after $\mathrm{NaOH}$ cleaning was around $90 \%$, $85 \%, 90 \%, 88 \%$, and $86 \%$ (\%SFR was $95-97 \%)$ for humic acid, BSA, alginate $\left(-\mathrm{Ca}^{+2}\right)$, 
alginate $\left(+\mathrm{Ca}^{+2}\right)$ and mixture respectively. For the polymeric UF, $\% \mathrm{R}_{\mathrm{cr}}$ after $\mathrm{NaOCl}$ cleaning was around $72 \%, 55 \%, 65 \%, 58 \%$ and $50 \%$ (\%SFR was $50-80 \%)$ whereas after $\mathrm{NaOH}$ cleaning was around $82 \%, 85 \%, 87 \%, 85 \%$ and $82 \%$ ( $\%$ SFR was $88-92 \%)$ for humic acid, BSA, alginate $\left(-\mathrm{Ca}^{+2}\right)$, alginate $\left(+\mathrm{Ca}^{+2}\right)$ and mixture respectively. Chemical cleanings of the polymeric UF showed similar $\% \mathrm{R}_{\text {cr }}$ trends to that observed for the ceramic UF, however, the overall $\% \mathrm{R}_{\text {cr r }}$ removal was always lower for the polymeric UF. This illustrate that chemical cleaning of the ceramic membrane was easier and more efficient than the polymeric membrane.

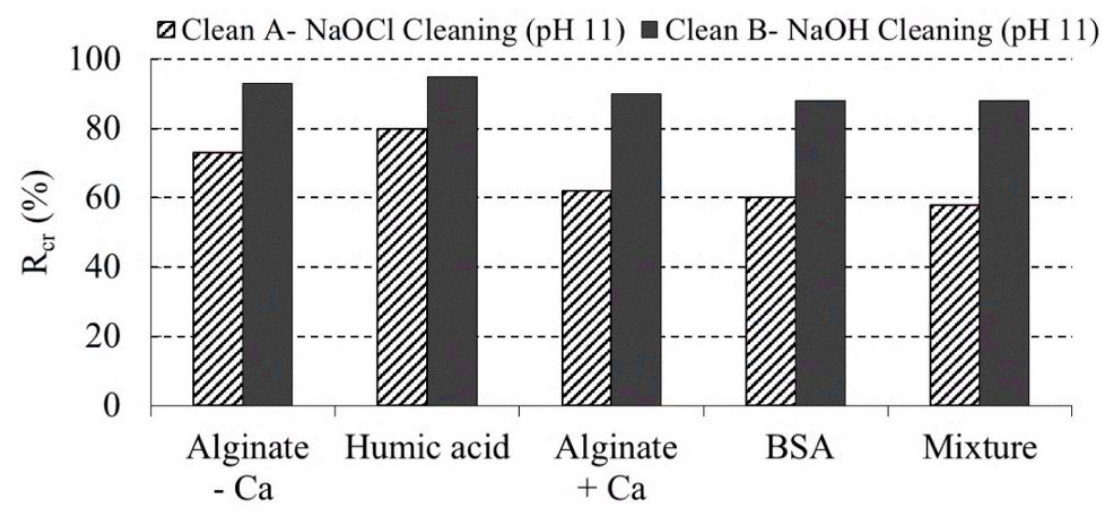

(A) Ceramic UF

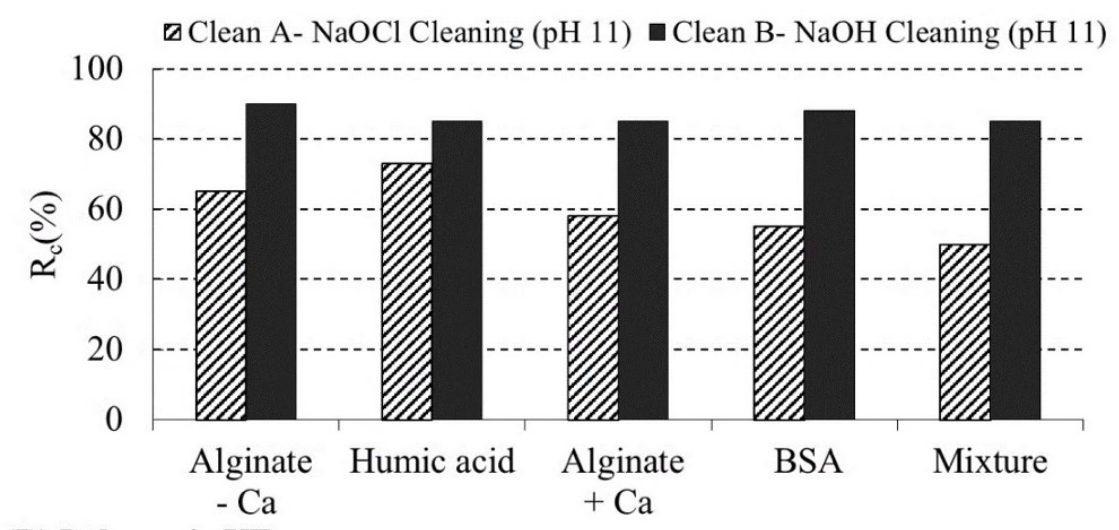

(B) Polymeric UF

Figure 6.7 - Cleaning efficiency based on the removal of chemically reversible resistance $\left(\mathrm{R}_{\mathrm{cr}}\right)$ - $\mathrm{pH} 11 ; 35^{\circ} \mathrm{C}$. (A) Ceramic UF; (B) polymeric UF 
6.3.2.2 Chemically reversible and irreversible carbon mass balance in wash waters Carbon mass (as percentages of total carbon) found in the $\mathrm{NaOCl}$ and $\mathrm{NaOH}$ wash waters (i.e. chemically reversible), and on the membrane after cleaning (i.e. chemically irreversible) are depicted in Figure 6.8. Surprisingly, it can be clearly seen that $\mathrm{NaOH}$ removed a higher carbon percentage of the BSA, alginate, and NOM mixture compared to $\mathrm{NaOCl}$. On the other hand, both $\mathrm{NaOCl}$ and $\mathrm{NaOH}$ removed a higher carbon percentage of humic acids. $\mathrm{NaOH}$ can result in a hydrolysis reaction of the proteins and polysaccharides wherein a hydrogen group is removed from the molecules and replaced by a salt, hence, resulting in strong electrostatic repulsion between protein molecules and membrane surface (Kuzmenko et al., 2005). Moreover, Fukuzaki (2006) reported that, the addition of $\mathrm{NaOCl}$ and $\mathrm{NaOH}$ to water increases surface tension of the $\mathrm{NaOCl}$ solution. This increase in surface tension prevents the $\mathrm{NaOCl}$ solution from wetting solid surfaces and from penetrating into the deposit layer (Fukuzaki, 2006). Perhaps this is why in this study $\mathrm{NaOH}$ removed the BSA and alginate off the membrane surface better than $\mathrm{NaOCl}$. More research is needed to better understand this unexpected difference in $\mathrm{NaOH}$ and $\mathrm{NaOCl}$ cleaning at the same $\mathrm{pH}$. This indicates that the cleaning efficiency of different chemicals is highly dependent on the type of foulants in feed water. Thus, the selection and characterization of chemical cleaners for membranes should be based on the source of NOM fouling rather than fouling type (i.e. organic fouling). The chemically irreversible carbon percentages (refer to Figure 6.8), that is the carbon on the membrane that remained after cleaning, were lower for the ceramic UF, which explains the high cleaning efficiency observed in Figure 6.7. Thus, the high efficiency of chemical cleaning can ultimately decrease the replacement rate and increase the operational lifetime expectancy of ceramic membranes, through the 
reduction of chemically irreversible fouling, compared to polymeric membranes. Future research is needed to estimate the long-term economic advantages of ceramic membranes compared to their polymeric counterpart.
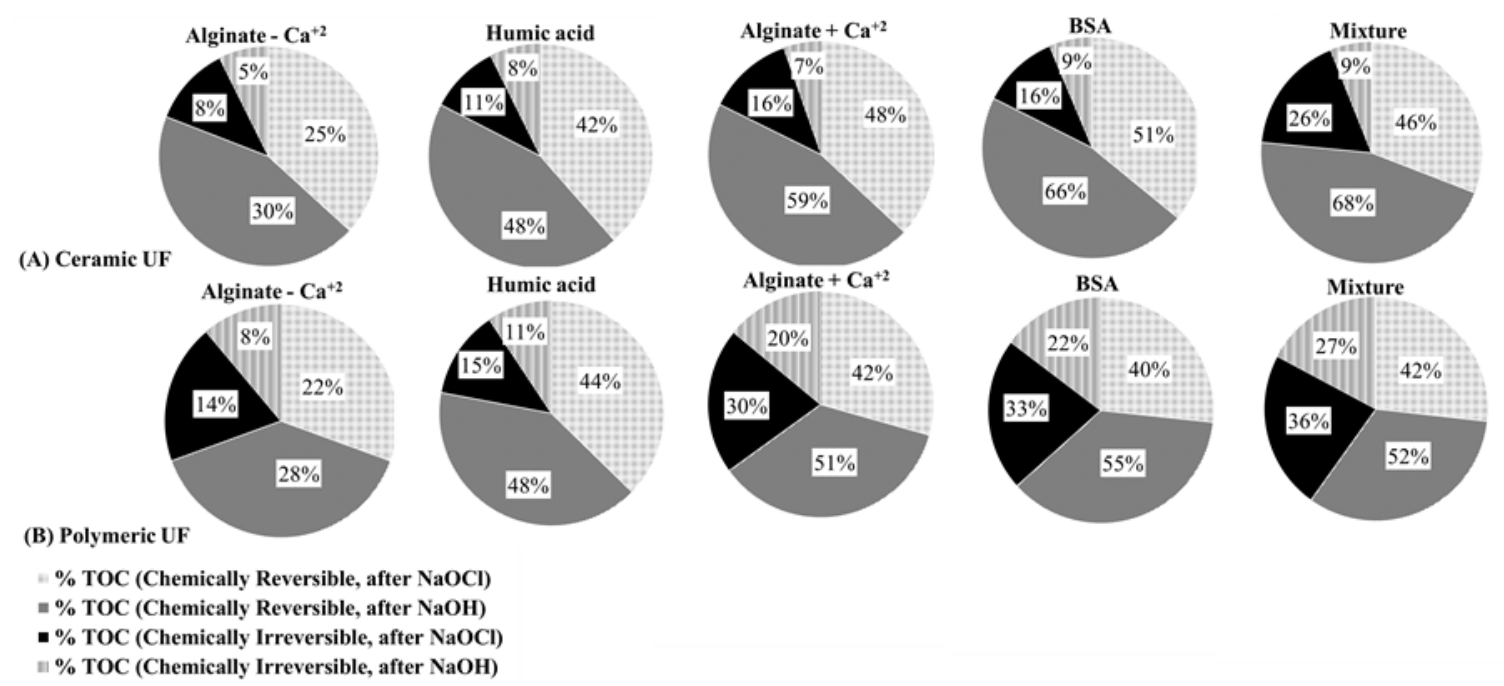

Figure 6.8 - Chemically reversible and irreversible \% carbon $\left(\mathrm{pH} 11 ; 35^{\circ} \mathrm{C}\right)$

FEEM plots for $\mathrm{NaOCl}$ and $\mathrm{NaOH}$ wash water for the solution with the NOM mixture are shown in Figure 6.9. The FEEM peaks found in the chemical wash water are quite similar, indicating that the type of NOM removed was similar under the two cleaning conditions. However, it can be observed that the fluorescent intensity is higher for the ceramic membrane, indicating that a larger portion of equivalent carbon was removed compared to the polymeric and indicates a better overall cleaning efficiency. The FEEM plots show similar results trend to that from RIS and carbon mass balance, demonstrating a useful approach for membrane fouling and cleaning evaluation when assessing removal of bulk organic constituents (i.e. humics and proteins). 

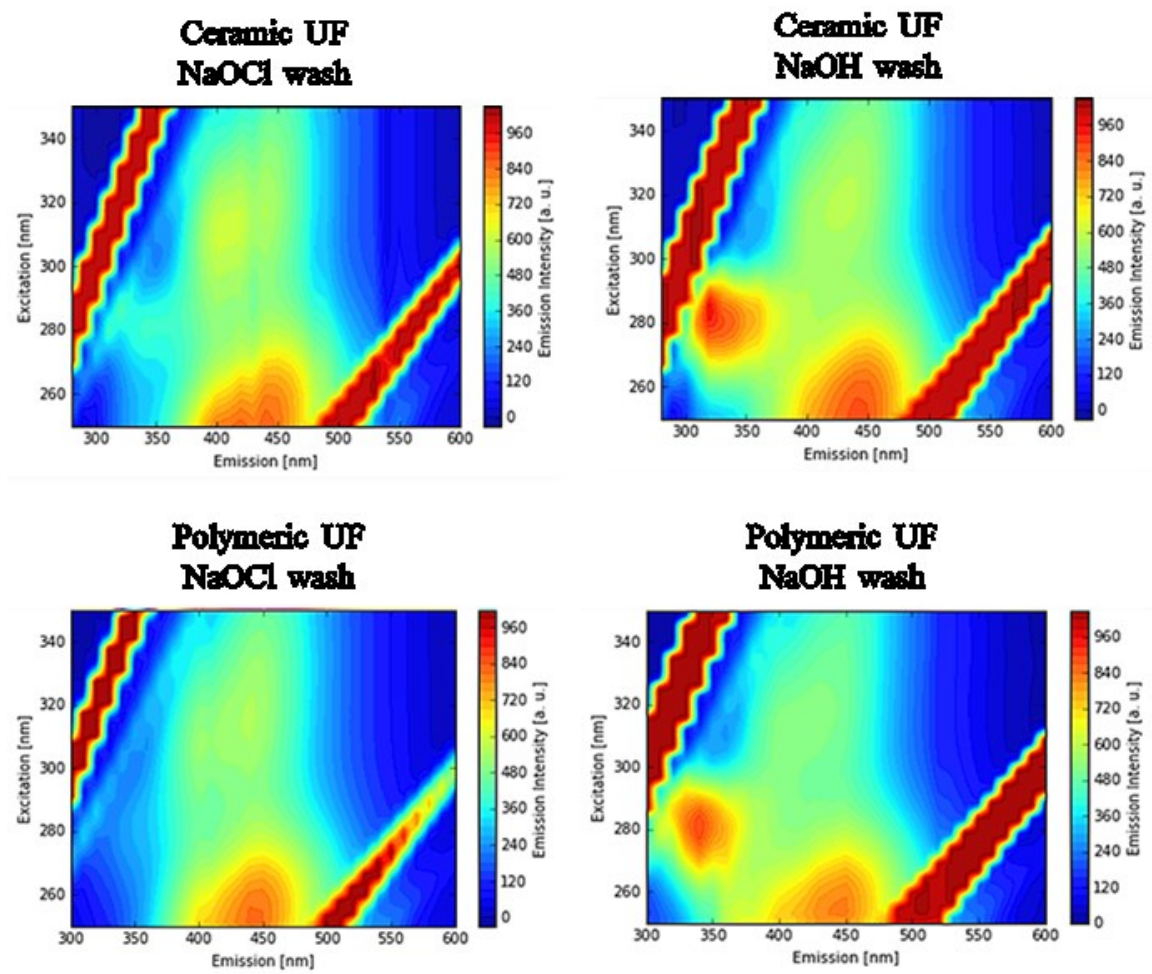

Figure 6.9 - FEEM plots of NOM mixture ( $\mathrm{NaOCl}$ and $\mathrm{NaOH}$ wash water)

\subsection{Conclusions}

Information on NOM fouling and cleaning behavior with respect to ceramic membranes in comparison to polymeric membranes is limited with respect to drinking water applications. This research demonstrated that ceramic membranes had a higher degree of reversible fouling compared to polymeric membranes, indicating better hydraulic cleaning. In addition, this research clearly demonstrated that the fouling order between the two membranes were similar highlighting the ease of integration of ceramic membranes into drinking water applications. Key points from the research are summarized here:

- The backwash efficiency $\left(\mathrm{R}_{\mathrm{hr}} / \mathrm{R}_{\mathrm{f}}\right.$ ratios) was $1.5 \mathrm{x}-2 \mathrm{x}$ higher for the ceramic UF in comparison to the polymeric UF. 
- Overall the hydraulic irreversibility was much higher for the polymeric membrane; where the NOM fouling resistance was $40-80 \%$ for the polymeric and only $20-50 \%$ for the ceramic membrane as determined by RIS.

- Fouling trends by RIS were confirmed by FEEM plots and the carbon content analysis in the ceramic UF backwash water was higher than the carbon content in the polymeric UF backwash water, which highlights an advantage of ceramic membranes with regards to hydraulic cleaning compared to polymeric membranes.

- More NOM was rejected by the ceramic membrane than the polymeric membrane although the fouling orders followed the same trend. The fouling was greatest with the NOM mixture $\approx \mathrm{BSA}$, alginate $\left( \pm \mathrm{Ca}^{+2}\right)$, and humic acid, respectively; indicating that both membranes are most susceptible to fouling by proteins.

- Chemical cleaning of ceramic and polymeric membranes was found to be effective for both membrane types. It was not possible to conclude that the ceramic membrane demonstrated an advantage for chemical cleaning. The FEEM plots demonstrate similar results trend to that from RIS and carbon mass balance, suggesting a useful approach for membrane fouling and cleaning evaluation when assessing removal of bulk organic constituents (i.e. humics and proteins).

\subsection{Acknowledgements}

The authors would like to acknowledge the Saudi Arabia Ministry of Education (MOE) and Natural Sciences and Engineering Research Council of Canada (NSERC) for helping fund this research. The authors wish to thank the CREDEAU laboratories at Polytechnique Montréal for providing the facilities to conduct this work as well as the collaboration of 
Mireille Blais. We also wish to thank Geoff Seatter and Kerwin Lewis for their help with installation of the automated filtration system.

\subsection{References}

Evans, P., Bird, M., Pihlajamaki, A., Nystrom, M., 2008. The influence of hydrophobicity, roughness and charge upon ultrafiltration membranes for black tea liquor clarification. Journal of Membrane Science 313, 250-262.

Fu, X., Maruyama, T., Sotani, T., Matsuyama, H., 2008. Effect of surface morphology on membrane fouling by humic acid with the use of cellulose acetate butyrate hollow fiber membranes. Journal of Membrane Science 320, 483.

Fukuzaki, S., 2006. Mechanisms of actions of sodium hypochlorite in cleaning and disinfection processes. Biocontrol Science, Vol. 11, No. 4, 147-157.

Gray, S.R., Ritchie, C.B., Tran, T., Bolto, B.A., 2007. Effect of NOM characteristics and membrane type on microfiltration performance. Water Research 41, 3833-3841.

Hashino, M., Hirami, K., Ishigmi, T., Ohmukai, Y., Maruyama, T., Kubota, N., Matsuyama, H., 2011. Effect of kinds of membrane materials on membrane fouling with BSA. Journal of Membrane Science 384, 157-165. 
Hashino, M., Hirami, K., Katagiri, M., Kubota, T., 2011. Effects of three natural organic matter types on cellulose acetate butyrate microfiltration membrane fouling. Journal of Membrane Science 379, 233-238.

Hofs, B., Ogier, J., Vries, D., Beerendonk, E., Cornelissen, E., 2011. Comparison of ceramic and polymeric membrane permeability and fouling using surface water. Separation and Purification Technology 79, 365-374.

Jamal, S., Chang, S., Zhou, H., 2014. Filtration behaviour and mechanisms of polysaccharides. Membranes 4, 319-332.

Jermann, D., Pronk, W., Boller, M., 2008. Mutual influences between natural organic matter and inorganic particles and their combined effect on ultrafiltration membrane fouling. Environmental Science and Technology 42, 9129-9136.

Katsoufidou, K., Yiantsios, S., Karabelas, A., 2005. A study of ultrafiltration membrane fouling by humic acids and flux recovery by backwashing: experiments and modeling. Journal of Membrane Science 266, 40.

Katsoufidou, K.S., Sioutopoulos, D.C., Yiantsios, S.G., Karabelas, A.J., 2010. UF membrane fouling by mixtures of humic acids and sodium alginate: Fouling mechanisms and reversibility. Desalination 264, 220-227. 
Kitis, M., Karanfil, T., Wigton, A., Kilduff, J., 2002. Probing reactivity of dissolved organic matter for disinfection by products formation using XAD-8 resin adsorption and UF fractionation. Water Research 36, 3834-3848.

Kuzmenko, D., Arkhangelsky, E., Belfer, S., Freger, V., Gitis, V., 2005. Chemical cleaning of UF membranes fouled by BSA. Desalination 179, 323-333.

Lee, N., Amy, G., Croûe, J.P., Buisson, H., 2005. Morphological analyses of natural organic matter (NOM) fouling of low-pressure membranes (MF/UF). Journal of Membrane Science 261,7 .

Lee, S., Kim, J., 2014. Differential natural organic matter fouling of ceramic versus polymeric ultrafiltration membranes. Water Research 48, 43-51.

Matilainen, A., Gjessing, E., Lathinen, T., Hed, L., Bhatnagar, A., Sillanpaa, M., 2011. An overview of the methods used in the characterisation of natural organic matter (NOM) in relation to drinking water treatment. Chemosphere 83, 1431-1442.

Montgomery, D., 2013. Design and analysis of experiments $8^{\text {th }}$ edition, John Wiley $\&$ Sons, Inc. Tempe, Arizona.

Nguyen, A., Tobiason, J., Howe, K., 2011. Fouling indices for low pressure hollow fiber membrane performance assessment. Water Research 45, 2627-2637. 
Pendergast, M., Hoek, E., 2011. A review of water treatment membrane nanotechnologies. Energy Environmental Science 4, 1946-1971.

Regula, C., Carretier, E., Wyart, Y., Gesan-Guiziou, G., Vincent, A. Boudot, D., Moulin, P., 2014. Chemical cleaning/disinfection and ageing of organic UF membranes: A review. Water Research 56, 325-365.

Shao, J., Hou, J., Song, H., 2011. Comparison of humic acid rejection and flux decline during filtration with negatively charged and uncharged ultrafiltration membranes. Water Research 45, 473-482.

Vasanth, D., Pugazhenthi, G., Uppaluri, R., 2013. Cross-flow microfiltration of oil-inwater emulsions using low cost ceramic membranes. Desalination 320, 86-95.

Wise, B., 2015. Comparative capital costs of ceramic and polymeric membranes. AWWA Membrane Technology Conference, Orlando, Florida.

Zhou, J.E., Chang, Q.B., Wang, Y.Q., Wang, J.M., Meng, G.Y., 2010. Separation of stable oil-water emulsion by the hydrophilic nano-sized $\mathrm{ZrO}_{2}$ modified $\mathrm{Al}_{2} \mathrm{O}_{3}$ microfiltration membrane. Separation and Purification Technology 75 (3), 243-248. 
Zularisam, A.W., Ahmad, A., Sakinah, M., Ismail, A.F., Matsuura, T., 2011. Role of natural organic matter (NOM), colloidal particles, and solution chemistry on ultrafiltration performance. Separation and Purification Technology 78, 189-20. 


\title{
Chapter 7
}

\section{Chemical Cleaning of Ceramic Ultrafiltration Membranes - Ozone versus Conventional Cleaning Chemicals}

\author{
Mohammad T. Alresheedi ${ }^{*}$, Onita D. Basu*, Benoit Barbeau ${ }^{* *}$ \\ * Department of Civil and Environmental Engineering, Carleton University, 1125 Colonel \\ By Drive, Ottawa, ON, K1S 5B6, Canada. \\ ${ }^{* *}$ Department of Civil, Geological and Mining Engineering, Polytechnique, C.P. 6079 \\ Succursale Centre-Ville, Montreal, QC, H3C 3A7, Canada. \\ Submitted to: Journal of Chemosphere
}

\begin{abstract}
This study investigates chemical cleaning mechanisms of a tubular ceramic UF membrane. The effect of cleaner type (ozone $\left(\mathrm{O}_{3}\right)$, sodium hypochlorite $(\mathrm{NaOCl})$ and sodium hydroxide $(\mathrm{NaOH})$ ), clean in place (CIP) $\mathrm{pH}$ (11 vs. 12), and cleaning sequence on the removal of irreversible fouling of hydrophobic (humic acids) and hydrophilic (alginate with and without calcium $\left.\left(\mathrm{Ca}^{+2}\right)\right)$ NOM fractions were investigated. Results showed that different NOM types responded differently to chemical cleaning. Alginate without $\mathrm{Ca}^{+2}$ and humic acids were equivalently removed by $\mathrm{NaOCl}$ or $\mathrm{NaOH}$ whereas a lower cleaning efficiency of alginate with $\mathrm{Ca}^{+2}$ was observed. Increasing the $\mathrm{pH}$ of $\mathrm{NaOCl}$ and $\mathrm{NaOH} \mathrm{CIP}$ increased the removal of the chemically reversible fouling index (UMFI $\left.{ }_{\text {cr }}\right)$. The efficiency of $\mathrm{NaOCl}$ was always lower than that of $\mathrm{NaOH}$ at the same $\mathrm{pH}$, which was attributed to surface tension $(\lambda)$ differences in the CIP water and potential differences in cleaning mechanism. The ceramic UF CIP cleaning using $\mathrm{O}_{3}\left(0.50 \mathrm{mg} \mathrm{O}_{3} / \mathrm{mgC}\right)$ for 1 hour
\end{abstract}


demonstrated higher cleaning efficiency for humic acids and alginate (with and without $\left.\mathrm{Ca}^{+2}\right),\left(\% \mathrm{UMFI}_{\mathrm{cr}}>98 \%\right)$, than $\mathrm{NaOCl}$ or $\mathrm{NaOH}$ alone $\left(\% \mathrm{UMFI}_{\mathrm{cr}}>80 \%\right)$. The $\mathrm{O}_{3} \mathrm{CIP}$ was as effective as 4 hours cleaning using a sequential $\mathrm{NaOH} / \mathrm{NaOCl}$ or combined $\mathrm{NaOCl}+\mathrm{NaOH} \mathrm{CIP}$.

Keywords: Carbon balance; Ceramic UF; Chemical cleaning; NOM fouling; Ozone CIP

\subsection{Introduction}

Membrane filtration with polymeric membranes has become increasingly common for drinking water treatment. More recently, ceramic membranes have attracted interest due to their hypothesized robustness to vigorous physical and chemical cleaning methods. However, fouling by natural organic matter (NOM) remains one of the major membrane challenges to maintain good membrane filtration performance (Hashino et al., 2011; Katsoufidou et al., 2010) and research on fouling and cleaning of NOM with ceramic membranes needs further investigation.

Membrane fouling is correlated to the ability of a membrane to reject NOM and the deposition of rejected organic constituents on its surface and/or in its pores (Kim et al., 2008). Reversible fouling can be removed by hydraulic backwash and/or chemical cleaning. Irreversible fouling resists hydraulic backwash and/or chemical cleaning. Irreversible fouling results in poor membrane performance by decreasing the permeate flux, and thus, increasing the need for chemical cleaning or even forcing membrane module replacement (Shi et al., 2014). 
The main goal of chemical cleaning is to remove membrane fouling, particularly irreversible fouling. The selection of a cleaning agent depends on the characteristics of feed water and compatibility of membrane materials (Regula et al., 2014). Sodium hypochlorite $(\mathrm{NaOCl})$ and sodium hydroxide $(\mathrm{NaOH})$ are the most commonly used chemicals in the maintenance and recovery cleaning of membranes (Wang et al., 2018; Shi et al., 2014). They are used to remove NOM fouling through oxidation, hydrolysis and solubilization (Regula et al., 2014). Oxidation by $\mathrm{NaOCl}$ degrades the NOM functional groups to carboxyl and aldehyde groups, which make them more susceptible to removal (Wang et al., 2014). On the other hand, $\mathrm{NaOH}$ encourages dissolution of carboxylic and phenolic functional groups such as proteins and polysaccharides (Porcelli and Judd, 2011). Studies addressing the impacts of different cleaning parameters of $\mathrm{NaOCl}$ and $\mathrm{NaOH}$ for cleaning polymeric membranes have shown that chemical concentration and cleaning sequence are important factors that governs the cleaning efficiency (Puspitasari et al., 2010; Hilal et al., 2005) Wang X. et al. (2018) found that increasing chlorine concentration from 100 to 1000 $\mathrm{mg} \mathrm{Cl} / \mathrm{L}$ of $\mathrm{NaOCl}$ resulted in faster diffusion of chlorine within the fouling layer, thus, higher oxidizing capability and cleaning efficiency. Puspitasari et al. (2010) reported that the repeated cleaning of PVDF membranes with $0.5 \% \mathrm{NaOCl}(5,000 \mathrm{mg} \mathrm{Cl} / \mathrm{L})$ was as effective as a single cleaning performed with $1 \%$. However, it is commonly known that a high concentration of chemicals and frequent cleaning can oxidize membrane polymers and cause swelling of membrane fibers, thus, shortening the membrane lifetime (Puspitasari et al., 2010; Arkhangelsky et al., 2007). Moreover, frequent cleaning can cause changes in membrane functional groups, physical structure and surface properties (Wang et al., 2018; Zhang et al., 2017; Shi et al., 2014). Thus, polymeric membranes are typically 
restricted to $\mathrm{pH} 11$ or lower and chlorine concentration $<500 \mathrm{mg} \mathrm{Cl} / \mathrm{L}$ (Regula et al., 2014; Porcelli et al., 2011).

Made of inorganic materials, ceramic membranes have many advantages over polymeric membranes as they are supposed to be mechanically, thermally, and chemically stable (Kim et al., 2008). Most importantly, ceramic membranes have superior chemical resistance allowing much more aggressive cleaning approaches (i.e. $\mathrm{pH}>11$ and high chemicals concentration) without risk of damaging membrane integrity (Lee et al., 2013). Utilization of high $\mathrm{pH}$ cleaning in a single or stepwise approach with the $\mathrm{NaOCl}$ and $\mathrm{NaOH}$ solutions can be beneficial for controlling irreversible fouling of ceramic membranes for drinking water treatment applications. However, to date there is little/no research in this area.

Ozone $\left(\mathrm{O}_{3}\right)$ cleaning adds in another dimension to cleaning of ceramic membranes that is not available to polymeric membranes. The use of $\mathrm{O}_{3}$ with ceramic membranes for the treatment of natural waters has been shown to reduce membrane fouling (Zhang et al., 2013; Kim et al., 2008). $\mathrm{O}_{3}$ can oxidize $\mathrm{NOM}$ through a direct molecular $\mathrm{O}_{3}$ attack as well as by an indirect means: free radical formation resulting from $\mathrm{O}_{3}$ decomposition in the bulk phase, $\mathrm{O}_{3}$ oxidation of $\mathrm{NOM}$ or $\mathrm{O}_{3}$ reaction with the ceramic membrane surface (Regula et al., 2014; Van Geluwe et al., 2011). Karnik et al. (2005) found that membrane flux improved by $65 \%$ - 90\% when a crossflow-operated ceramic membrane was combined with ozonation. Other studies such as Zhang et al. (2013) and Sartor et al., (2008) reported that in situ ozonation transforms organics into smaller hydrophilic molecules, thus, less fouling. It should be noted that in the above studies, $\mathrm{O}_{3}$ is dosed in a pre-ozonation mode, 
with the backwash step or continuously injected into the membrane tank. However, limited to no research exists that investigate alternative ozone clean in place $\left(\mathrm{O}_{3} \mathrm{CIP}\right)$ cleaning regimes with respect to NOM fouling control for ceramic membranes.

Research which examines CIP methodologies with different NOM fractions is required to increase operational understanding of cleaning regimes that control irreversible fouling of ceramic membranes. Therefore, in this paper, we investigated various chemical cleaning conditions of a ceramic UF membrane fouled by hydrophilic (alginate) and hydrophobic (humic acids) NOM fractions. The NOM fouling behavior was characterized using the unified membrane fouling index (UMFI), NOM retention and carbon mass balance to assess for respective differences in reversible and irreversible fouling mechanisms. $\mathrm{O}_{3} \mathrm{CIP}$ performance was investigated for controlling irreversible alginate and humic acids fouling in comparisons to a single, stepwise, or combined chemical cleaning approach using $\mathrm{NaOCl}$ and $\mathrm{NaOH}$.

\subsection{Materials and Methods}

\subsubsection{Model foulants}

NOM model foulants used in this study were a hydrophobic NOM, humic acids (Sigma Aldrich lot \#BCBK5107V) (2.5 mg C/L) and a hydrophilic polysaccharide, sodium alginate, (Sigma Aldrich) $(2.5 \mathrm{mg} \mathrm{C} / \mathrm{L})$; and sodium alginate with $\mathrm{Ca}^{+2}$ addition $75 \mathrm{mg} / \mathrm{L}$ as $\mathrm{CaCO}_{3}\left(30 \mathrm{mg} \mathrm{Ca}^{+2} / \mathrm{L}\right)$ using calcium sulfate $\left(\mathrm{CaSO}_{4}\right)$. Humic acids and alginate (Sigma Aldrich) have been previously used in numerous membrane fouling investigations because of their fouling propensity and known characteristics (Katsoufidou et al., 2010; Xiao et al., 
2009). The test with alginate/ $\mathrm{Ca}^{+2}$ was included in the experimental design due to the high fouling propensity of this combination (Jamal et al., 2014). Model solutions were prepared using pure deionized (DI) water and were mixed using a magnetic stirrer one-day prior to experiments ensuring that materials were dissolved completely. In addition, turbidity (5 $\mathrm{mg} / \mathrm{L}$ of kaolin clay, equivalent to $5 \mathrm{NTU}$, with $>95 \%$ of particles in the $2-5 \mu \mathrm{m}$ range), and alkalinity $\left(75 \mathrm{mg} / \mathrm{L}\right.$ as $\left.\mathrm{CaCO}_{3}\right)$ using sodium bicarbonate $\left(\mathrm{NaHCO}_{3}\right)$ were added. The $\mathrm{pH}$ of the feed solution was maintained around 7.5. The zeta potential of humic acids and alginate feed solutions were measured using the Malvern Zetasizer-Nano.

\subsubsection{Experimental setup and approach}

An automated ceramic membrane system (Figure 7.1) was used for this research. The system consists of a digital gear pump (Cole Parmer: Drive no. 75211-30, Head no. 0700304), pressure transducer (Omega), flow meter (Cole Parmer: model no. 32703-52), solenoid valves (MacMaster), and pressure vessel (Cole Parmer). Nitrogen gas was used to maintain pressure in the pressure vessel for backwash. Labview program (National Instruments, NI) code was created to operate the system automatically such as controlling the feed pump to have a constant flux, and automatically switches from filtration cycles to backwash cycles while recording flux and pressure data. A tubular ceramic UF membrane $\left(\right.$ Ceramem ${ }^{\mathrm{TM}}$, Veolia) was used and its characteristics are as follows: monolithic module, $\mathrm{SiC}$ membrane material, inside-out channels (61), square channel $(2 \mathrm{~mm})$, pore size $(0.01 \mu \mathrm{m})$, area $(0.1$ $\mathrm{m}^{2}$ ), and module length $(300 \mathrm{~mm})$. The ceramic membrane was fouled by humic acids or alginate for 24 hours in dead-end filtration mode under constant flux of $100 \mathrm{LMH}$; backpulses were conducted for 20 seconds every 4 hours using pressurized water at 2 bars 
( $\sim 30 \mathrm{psig})$. Between cleaning experiments, a clean water flux (CWF) test was performed to determine the membrane resistance using the transmembrane pressure (TMP) versus flux relationship for a clean membrane. This was done by filtering water through the ceramic membrane at a low flux and recording the TMP after 30 minutes the flux was increased, and the procedure continued in a stepwise fashion. The initial membrane flux $\left(\mathrm{J}_{\text {initial }}\right)$, and TMP $(\Delta \mathrm{P})$ and kinematic viscosity $(\mu)$ were used to estimate membrane resistance $\left(\mathrm{R}_{\mathrm{m}}\right)$ using the Equation 7.1.

$\mathrm{R}_{\mathrm{m}}=\frac{\Delta \mathrm{P}}{\mu \mathrm{J}_{\text {initial }}} \quad$ Equation (7.1)

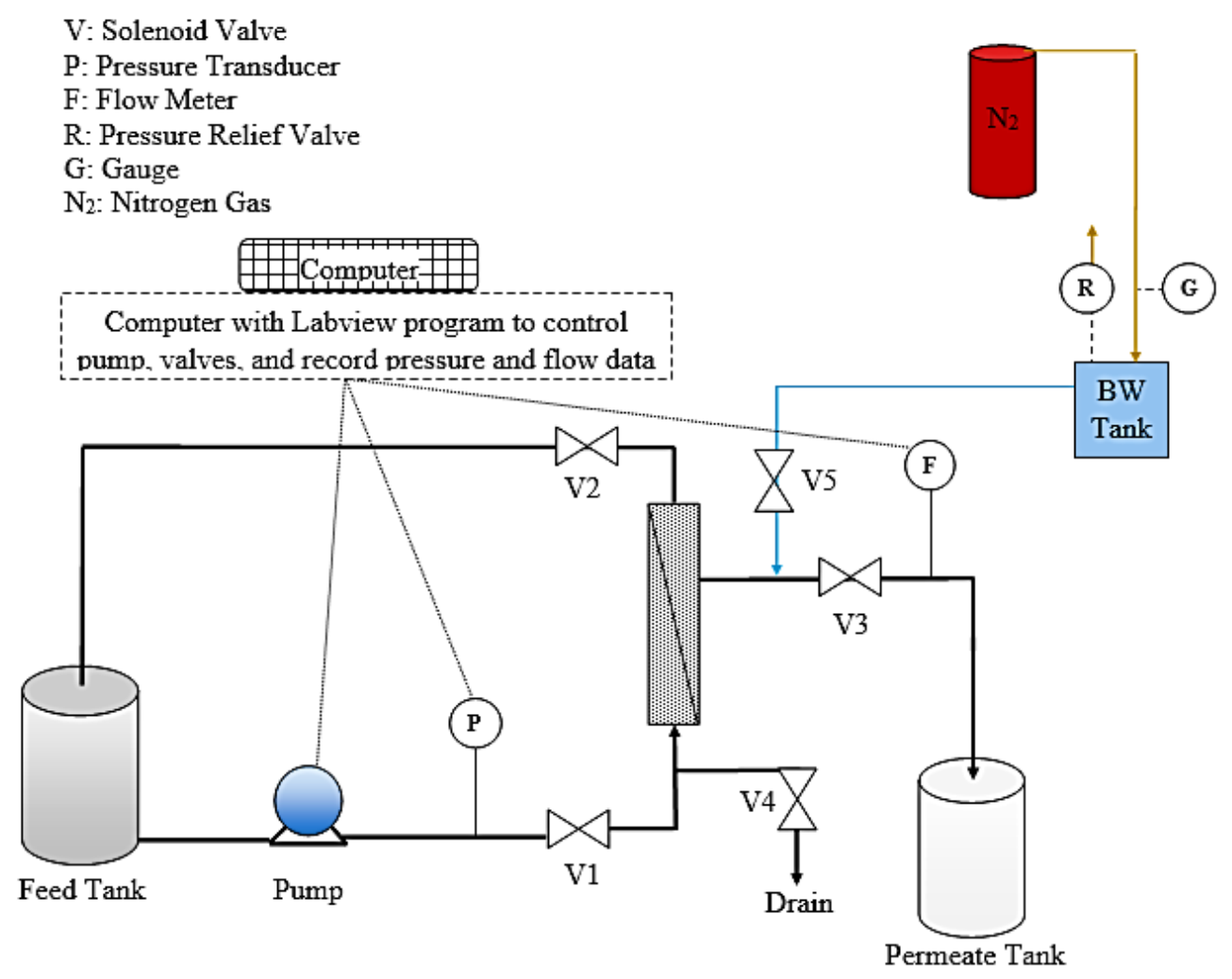

Figure 7.1- Automated ceramic membrane system 


\subsubsection{Chemical cleaning experiments}

This research investigates $\mathrm{pH} 12$ solutions in a single, stepwise, or combined approach with $\mathrm{NaOCl}$ and $\mathrm{NaOH}$ and $\mathrm{O}_{3} \mathrm{CIP}$ for increased irreversible fouling control on ceramic membranes for drinking water treatment applications. Both $\mathrm{pH} 12$ and $\mathrm{O}_{3} \mathrm{CIP}$ are not recommended with polymeric membranes and could highlight an advantage to ceramic membranes in drinking water applications for irreversible fouling control.

\subsubsection{Cleaning $\mathrm{pH}$ effect (pH 11 vs. $\mathrm{pH} 12$ )}

Chemical cleaning (CIP) was performed using two common membrane cleaners: an oxidant, $\mathrm{NaOCl}$ (500 mg Cl $2 / \mathrm{L}$, as free chlorine) or caustic, $\mathrm{NaOH}$. $\mathrm{NaOCl}$ was purchased as a solution $(6 \%)$. The concentration of free chlorine in the wash water was measured before testing using the $\mathrm{HACH}$ method (8021) and a digital colorimeter ${ }^{\mathrm{TM}} \mathrm{II}$. $\mathrm{NaOCl}$ and $\mathrm{NaOH}$ cleaning was examined at two different $\mathrm{pH}$ of 11 and 12 . Cleaning was performed alternatively for 4 hours at a cleaning water temperature of $35^{\circ} \mathrm{C}$. Chemicals were prepared in $4 \mathrm{~L}$ of DI water and recirculated in a closed loop at a cross flow velocity of $0.1 \mathrm{~m} / \mathrm{s}$ for 1 hour followed by 3 hours soak.

7.2.3.2 Chemicals cleaning sequence $(\mathrm{NaOCl} / \mathrm{NaOH}, \mathrm{NaOH} / \mathrm{NaOCl}$, combined $\mathrm{NaOCl}+\mathrm{NaOH})$

The effect of chemical cleaning sequence of the ceramic UF was examined using three options. Option A (NaOCl/NaOH): cleaning with $500 \mathrm{mg} \mathrm{Cl}_{2} / \mathrm{L} \mathrm{NaOCl}(\mathrm{pH} 12)$ for 2 hours followed by $\mathrm{NaOH}$ (pH 12) for 2 hours (total of 4 hours). Option $\mathbf{B}$ (NaOH/NaOCI): cleaning with $\mathrm{NaOH}(\mathrm{pH}$ 12) for 2 hours followed by $500 \mathrm{mg} \mathrm{Cl} / 2 \mathrm{~L} \mathrm{NaOCl}(\mathrm{pH} 12)$ for 2 
hours (total of 4 hours). Option $\mathbf{C}(\mathbf{N a O C l}+\mathbf{N a O H})$ : $\mathrm{NaOCl}$ was simultaneously mixed with $\mathrm{NaOH}(\mathrm{pH} 12)$ i.e. both chemicals were added concurrently at the same concentration as in their single solution (500 $\mathrm{mg} \mathrm{Cl} 2 / \mathrm{L} \mathrm{NaOCl}+400 \mathrm{mg} \mathrm{NaOH} / \mathrm{L}$ ), and the cleaning duration was maintained at a 4 hours duration to ensure the same chemical contact time for direct comparisons with individual cleaning solutions. Cleaning was performed at $35^{\circ} \mathrm{C}$ water temperature.

\subsubsection{Ceramic UF cleaning using $\mathrm{O}_{3} \mathrm{CIP}$}

$\mathrm{O}_{3}$ cleaning efficiencies for hydrophilic NOM, alginate with and without $\mathrm{Ca}^{+2}$, and hydrophobic NOM, humic acids, were examined. For each cleaning experiment, 4 L of ozonated water was first prepared by bubbling at $4{ }^{\circ} \mathrm{C}$ a $6 \%$ ozone/oxygen gas (Ozone Solutions, USA) in DI water to saturation. Cleaning was performed by recirculating $\mathrm{O}_{3}$ injected at either $0.25 \mathrm{mg} \mathrm{O}_{3} / \mathrm{mg} \mathrm{C}$ or $0.50 \mathrm{mg} \mathrm{O} 3 / \mathrm{mg} \mathrm{C}$, where $\mathrm{mg} \mathrm{C}$ is the carbon mass accumulated on the membrane surface (i.e., $\mathrm{O}_{3}$ concentration in the $4 \mathrm{~L}$ cleaning solution ranges from $15-30 \mathrm{mg} \mathrm{O}_{3} / \mathrm{L}$ and $30-60 \mathrm{mg} \mathrm{O}_{3} / \mathrm{L}$ for the 0.25 and $0.50 \mathrm{mg} \mathrm{O}_{3} / \mathrm{mg} \mathrm{C}$ ratios respectively). Recirculation of $\mathrm{O}_{3}$ was performed in a closed loop for 1 hour at a cleaning water temperature of $15{ }^{\circ} \mathrm{C} . \mathrm{O}_{3}$ residuals were measured using the standard indigo trisulfonate method (Bader and Hoigin, 1981). Total organic carbon (TOC) analyses were performed during $\mathrm{O}_{3}$ cleaning by collecting a sample at different time intervals (i.e. 1, 5, 10 minutes, etc.) to determine the cumulative $\mathrm{O}_{3}$ demand and irreversible carbon removal as a function of cleaning time. 


\subsubsection{Analysis}

7.2.4.1 The unified membrane fouling index (UMFI)

Fouling and cleaning were assessed using the UMFI model as shown in Equation 7.2 (Huang et al., 2008). Where $\mathrm{J}_{\mathrm{s}}$ ' is the normalized specific flux (i.e. measured specific flux $\left(\mathrm{J}_{\mathrm{s}}\right)$ divided by initial specific flux $\left(\mathrm{J}_{\mathrm{s} 0}\right)$, (unitless), UMFI is an estimate of the extent of fouling $\left(\mathrm{m}^{-1}\right)$, and $\mathrm{V}_{\mathrm{S}}\left(\mathrm{m}^{3} / \mathrm{m}^{2}\right)$ is the specific permeate volume.

$\frac{1}{\mathrm{~J}_{\mathrm{s}}{ }^{\prime}}=1+(\mathrm{UMFI}) \mathrm{x} \mathrm{Vs}$

Equation (7.2)

Total fouling resistance index $\left(\mathrm{UMFI}_{\mathrm{f}}\right)$ can be divided into the hydraulically reversible fouling resistance index (UMFI $\mathrm{hr}_{\mathrm{r}}$ (i.e., removed by backwash), and hydraulically irreversible fouling resistance index (UMFI $\left.{ }_{h i r}\right)$, (i.e., remained after backwash), as shown in Equation 7.3. The $\mathrm{UMFI}_{\text {hir }}$ can be divided into $\mathrm{UMFI}_{\mathrm{cr}}$ and $\mathrm{UMFI}_{\mathrm{cir}}$. $\mathrm{UMFI}_{\mathrm{cr}}$ is chemically reversible fouling resistance index (i.e., removed by chemical cleaning); and $\mathrm{UMFI}_{\mathrm{cir}}$ is chemically irreversible fouling resistance index (i.e. remained after chemical cleaning).

$U M F I_{f}=U M F I_{h r}+U M F I_{h i r}=U M F I_{h r}+U M F I_{c r}+U M F I_{c i r}$

Equation (7.3)

$\mathrm{UMFI}_{\mathrm{f}}$ was calculated using the slope of the linear region of $1 / \mathrm{J}_{\mathrm{s}}$ ' versus $\mathrm{V}_{\mathrm{s}}$ graph from the start to end of filtration. $\mathrm{UMFI}_{\text {hir }}$ was calculated using the slope of $1 / \mathrm{J}_{\mathrm{s}}$ ' versus $\mathrm{V}_{\mathrm{s}}$ graph using data points immediately after backwash. $\mathrm{UMFI}_{\mathrm{hr}}$ is the difference between $\mathrm{UMFI}_{\mathrm{f}}$ and $\mathrm{UMFI}_{\text {hir. }} \mathrm{UMFI}_{\text {cir }}$ was calculated using the slope of $1 / \mathrm{J}_{\mathrm{s}}$ 'versus $\mathrm{V}_{\mathrm{s}}$ graph using data 
points immediately after the chemical cleaning step. $\mathrm{UMFI}_{\mathrm{cr}}$ is the difference between $\mathrm{UMFI}_{\text {hir }}$ and $\mathrm{UMFI}_{\mathrm{cir}}$.

\subsubsection{Carbon mass balance}

Carbon mass balance was performed to determine indirectly the carbon mass remaining on the membrane before and after each cleaning step. This was performed by TOC analysis on feed, permeate, hydraulic backwash and chemical wash waters. The mass of chemically irreversible carbon was deducted using Equation 7.4. The \% error of the carbon measurements was calculated to ensure accurate mass balance at different testing conditions. Carbon data was then compared to the fouling resistance data estimated by the UMFI method.

Mass (cir) $=$ Mass (Feed) - Mass (Permeate) -Mass (hr) - Mass (cr) Equation (7.4)

Where: Mass(cir) (chemically irreversible carbon mass, i.e. remained on membrane); $\operatorname{Mass}(\mathrm{hr})_{\text {(hydraulically reversible carbon mass, i.e. in backwash water); Mass(cr) }}$ (chemically reversible carbon mass, i.e. in chemical wash water).

\subsubsection{Surface tension $(\lambda)$ measurements}

Surface tension measurements, using the capillary rise method, of $\mathrm{NaOCl}$ and $\mathrm{NaOH} \mathrm{CIP}$ waters were conducted by immersing a capillary tube into the CIP water and measuring the liquid height in the tube. Jurin's law (Equation 7.5) was used to estimate the surface tension of CIP chemicals. 
$\lambda=\frac{\text { h.p.g } \cdot r}{2 \cos \theta}$

Equation (7.5)

Where $\lambda$ is the surface tension $(\mathrm{N} / \mathrm{m})$; $\mathrm{h}$ is the height of the liquid in the capillary tube $(\mathrm{m})$; $\rho$ is the liquid density $\left(\mathrm{kg} / \mathrm{m}^{3}\right) ; \mathrm{g}$ is the gravitational acceleration $\left(\mathrm{m} / \mathrm{s}^{2}\right) ; \mathrm{r}$ is the tube radius (m); $\theta$ is the contact angle. The contact angle was determined by taking photos of a water/chemical droplets and using ImageJ software (an imaging processing software) to determine the contact angle. Photos were taken 1 minute after depositing the drop on the surface. Tests were conducted in triplicate for each CIP condition and the average of three measurements is reported. The $\lambda$ of $\mathrm{NaOCl}$ and $\mathrm{NaOH}$ CIP waters at $\mathrm{pH} 11$ was further measured with the addition of nonionic surfactants $(500 \mathrm{mg} / \mathrm{L}$ of Tween 80$)$ to assess the impact of surfactants addition on the wetting ability of $\mathrm{NaOCl}$ and $\mathrm{NaOH}$.

\subsection{Results and Discussion}

\subsubsection{Ceramic UF fouling by alginate and humic acids}

Initial filtration experiments were conducted to establish the fouling behavior of hydrophobic (humic acid) and hydrophilic (sodium alginate) NOM with ceramic UF membrane. The UMFI (Huang et al., 2008) which represents the slope of the data in the linear zone, was used to quantify fouling of each NOM type. Figure 7.2 shows that the membrane fouling behaviors differed between the model NOM solutions. The alginate + $\mathrm{Ca}^{+2}$ solution resulted in the highest fouling $\left(\mathrm{UMFI}_{\text {alginate }+\mathrm{Ca}}{ }^{+2}\right.$ of $\left.1.13 \mathrm{~m}^{-1}\right)$ followed by

alginate $-\mathrm{Ca}^{+2}\left(\mathrm{UMFI}_{\text {alginate-Ca }}{ }^{+2}\right.$ of $\left.0.89 \mathrm{~m}^{-1}\right)$, and humic acids $\left(\mathrm{UMFI}_{\text {humic acids }}\right.$ of $\left.0.71 \mathrm{~m}^{-1}\right)$ respectively. The rate of fouling increased with each filtration cycle, demonstrating high 
accumulation of NOM. The presences of $\mathrm{Ca}^{+2}$ in feed water showed negative impacts on alginate fouling. Specifically, after the first 8 hours filtration cycle, the alginate $+\mathrm{Ca}^{+2}$ solution demonstrated higher increase in fouling compared to alginate $-\mathrm{Ca}^{+2} \cdot \mathrm{Ca}^{+2}$ reduced the zeta potential of alginate from $-36 \mathrm{mV}$ to $-20 \mathrm{mV}$ due to double layer compression and reduction of the electrostatic repulsion forces between colloids and membrane surface, thus increasing the fouling rate. This can be supported by Katsoufidou et al. (2010) study on alginate fouling with polymeric membranes. It was found that the presence of calcium leads to reduced alginate inter-chain electrostatic repulsion, thus promoting formation of coiled alginate macro-molecular structures. Such coiled macro-molecules tend to form more compact and less permeable fouling layers. The results from the ceramic UF system analogous to those by Katsoufidou et al. (2010) and Hashino et al. (2011) using polymeric membranes, in which humic acid had a gradual flux decline and lower fouling rate compared to alginate.

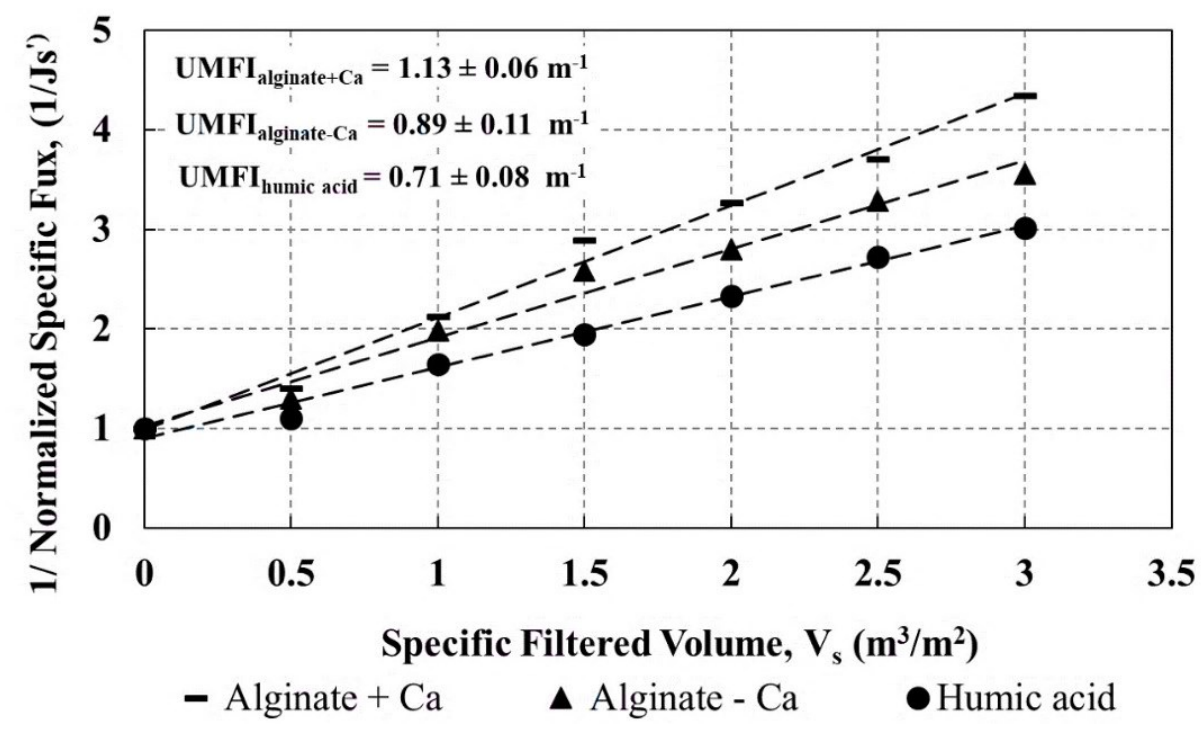

Figure 7.2 -Ceramic UF membrane fouling by different NOM solutions (Constant Flux: $100 \mathrm{LMH}$, BW: every 4 hours $\left(0.4 \mathrm{~m}^{3} / \mathrm{m}^{2}\right)$ for $20 \mathrm{~s}$ at $30 \mathrm{psi} . \mathrm{pH}=7.5$. Total filtration time: 24 hours. UMFI: Unified membrane fouling index. 
Figure 7.3 shows the hydraulically reversible and irreversible fouling ratios (i.e. $\mathrm{UMFI}_{\mathrm{hr}} / \mathrm{UMFI}_{\mathrm{f}}$ and $\left.\mathrm{UMFI}_{\text {hir }} / \mathrm{UMFI}_{\mathrm{f}}\right)$ for the alginate $\pm \mathrm{Ca}^{+2}$ and humic acids solutions. Overall, the $\mathrm{UMFI}_{\text {hir }} / \mathrm{UMFI}_{\mathrm{f}}$ ratio of different NOM fractions ranged between $16 \%-41 \%$ of total fouling. The alginate solution showed different fouling behaviors with and without $\mathrm{Ca}^{+2}$ addition. Fouling was more reversible for the alginate $-\mathrm{Ca}^{+2}$ solution in which $76 \%$ was removed by backwash whereas in the case of alginate $+\mathrm{Ca}^{+2}$, only $59 \%$ was removed. As a result, deposition of alginate onto the membrane surface increases and a more densely packed fouling layer of high hydraulic resistance was formed. Moreover, alginate (with and without $\mathrm{Ca}^{+2}$ ) resulted in higher irreversible fouling compared to humic acids indicating a strong influence of polysaccharides on ceramic membrane fouling compared to humic substances. Katsoufidou et al. (2010) and Kimura et al. (2004) studies with polymeric UF membranes showed that polysaccharides caused irreversible fouling apparently due to pore blockage. It was reported that alginate resulted in a rapid irreversible fouling due to internal pore constriction which led to development of cake layer of high specific resistance (Katsoufidou et al., 2010; Kimura et al., 2004).

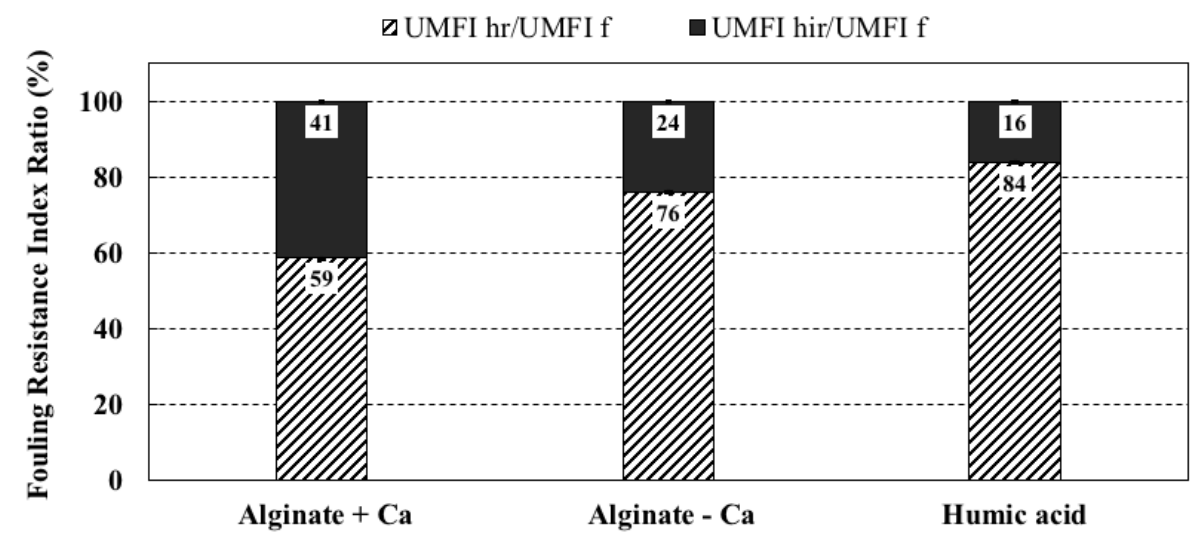

Figure 7.3 - Hydraulically reversible fouling index $\left(\mathrm{UMFI}_{\mathrm{hr}}\right)$ and hydraulically irreversible fouling index (UMFI $\mathrm{Uir}_{\mathrm{hir}}$ ) ratios*

*Higher $\mathrm{UMFI}_{\mathrm{hr}} / \mathrm{UMFI}_{\mathrm{f}}$ percentage indicates higher backwash efficiency and lower irreversibility. 
Table 7.1 presents the carbon mass found in the backwash water (hydraulically reversible), remaining on the membrane (hydraulically irreversible) and in the permeate water. During the filtration of alginate $\left(-\mathrm{Ca}^{+2}\right), 54 \%$ of feed carbon $\left(0.22 \mathrm{mg} \mathrm{C} / \mathrm{cm}^{2}\right)$ remained on the ceramic membrane compared to $64 \%\left(0.35 \mathrm{mg} \mathrm{C} / \mathrm{cm}^{2}\right)$ for alginate $\left(+\mathrm{Ca}^{+2}\right)$, which demonstrate the effect of $\mathrm{Ca}^{+2}$ on fouling reversibility/irreversibility of alginate. For humic acids, $36 \%$ of feed carbon $\left(0.18 \mathrm{mg} \mathrm{C} / \mathrm{cm}^{2}\right)$ remained on the membrane which was lower than carbon mass determined for alginate $\pm \mathrm{Ca}^{+2}$. Therefore, membrane backwash was more effective in removing humic acids fouling deposits compared to alginate (refer to Table 7.1). The results of the carbon masses are coherent with the UMFI data in Figure 7.3. The higher $\%$ of the hydraulically reversible carbon (i.e., carbon in the backwash water) for humic acids indicate higher backwash efficiency (i.e. high $U M F I_{h r} / U_{M F I}$ ratio) and lower irreversibility compared to Alginate $-\mathrm{Ca}^{+2}$ (as reflected in Figure 7.3). Additionally, the high hydraulically irreversible carbon (i.e., carbon remained on the membrane) for the alginate with $\mathrm{Ca}^{+2}$ addition (i.e., Alginate $+\mathrm{Ca}^{+2}$ ) indicates higher irreversible fouling and thus lower backwash efficiency (refer to Figure 7.3). Thus, carbon mass balance measurements demonstrated a simple and useful approach for fouling quantification in bench scale setting.

Table 7.1- Hydraulically reversible and irreversible carbon percentages $(n=3) *$

\begin{tabular}{cccc}
\hline Model NOM & $\begin{array}{c}\text { Hydraulically } \\
\text { Reversible Carbon } \\
(\%)\end{array}$ & $\begin{array}{c}\text { Hydraulically } \\
\text { Irreversible } \\
\text { Carbon } \% * *\end{array}$ & $\begin{array}{c}\text { Permeate } \\
\text { Carbon (\%) }\end{array}$ \\
\hline Humic acids & $52 \pm 6$ & $36 \pm 8(0.18)$ & $12 \pm 6$ \\
Alginate $-\mathrm{Ca}^{+2}$ & $36 \pm 4$ & $54 \pm 8(0.22)$ & $10 \pm 5$ \\
Alginate $+\mathrm{Ca}^{+2}$ & $28 \pm 8$ & $64 \pm 6(0.35)$ & $8 \pm 6$ \\
$*$ Total organic carbon in the feed: $100 \% . \mathrm{n}=3$ : triplicate experiments. & \\
$* *$ Values in parenthesis are giving the surface concentration of carbon in $\mathrm{mg} / \mathrm{cm}^{2}$ &
\end{tabular}




\subsubsection{Ceramic UF cleaning: effect of cleaning $\mathrm{pH}$ and cleaning agent}

The fouling experiments clearly demonstrated that alginate $\pm \mathrm{Ca}^{+2}$ and humic acids contribute differently to irreversible fouling of a ceramic UF membrane. Therefore, the next step was to compare the cleaning efficiency of $\mathrm{NaOCl}, \mathrm{NaOH}$, and $\mathrm{O}_{3} \mathrm{CIP}$ for controlling irreversible fouling of alginate $\pm \mathrm{Ca}^{+2}$ and humic acids. The effect of $\mathrm{pH}$ on the cleaning efficiency of $\mathrm{NaOCl}$ and $\mathrm{NaOH}$ is shown in Figure 7.4. The percentages of $\mathrm{UMFI}_{\text {cr }}$ recovered by $\mathrm{NaOCl} \mathrm{CIP}(500 \mathrm{mg} \mathrm{Cl} / \mathrm{L}$ ) at $\mathrm{pH} 11$ were $62 \%, 74 \%$, and $80 \%$ for alginate $+\mathrm{Ca}^{+2}$, alginate $-\mathrm{Ca}^{+2}$, and humic acids, respectively. On the other hand, $\mathrm{NaOH}$ showed much higher cleaning efficiency at $\mathrm{pH} 11$ compared to $\mathrm{NaOCl}$ in which the $\% \mathrm{UMFI}_{\text {cr }}$ recovered were $80 \%, 86 \%$, and $88 \%$ for alginate $+\mathrm{Ca}^{+2}$, alginate $-\mathrm{Ca}^{+2}$, and humic acids respectively. The cleaning efficiency of $\mathrm{NaOCl}$ increased significantly $(\mathrm{p}<$ 0.05 ) by $10-20 \%$ with increasing $\mathrm{pH}$ from 11 to 12 while there was also a significant $\mathrm{p}<$ 0.05 ) $10 \%$ improvement for $\mathrm{NaOH}$ at the higher $\mathrm{pH}$ of 12 . Increasing $\mathrm{pH}$ can cause swelling of the fouling layer which results in an increase in the cake voidage, and thus results in a higher cleaning efficiency (Wang et al., 2018; Strugholtz et al., 2005). The increase in the cleaning ability of $\mathrm{NaOCl}$ and $\mathrm{NaOH}$ at $\mathrm{pH} 12$ in this study resulted in a more effective oxidation and hydrolysis reactions between cleaning chemicals and NOM within the fouling layer (Shi et al., 2011). Consequently, the fouling layer was broken down relatively more easily at $\mathrm{pH} 12$ compared to $\mathrm{pH}$ of 11 and resulted in a higher cleaning efficiency. Liang et al. (2008) study indicates that caustic promotes a more open fouling layer that allows the chlorine to reach the membrane surface, which explain the increased efficacy of $\mathrm{NaOCl}$ at higher $\mathrm{pH}$ of 12 compared to 11 . 
Figure 7.4 also shows the $\% \mathrm{UMFI}_{\text {cr }}$ recovered by ozone $\left(\mathrm{O}_{3}\right)$ cleaning compared to $\mathrm{NaOCl}$ and $\mathrm{NaOH}$. It can be clearly seen that a 1 hour of $\mathrm{O}_{3} \mathrm{CIP}$ at a ratio of $0.50 \mathrm{mgO}_{3} / \mathrm{mgC}(\sim$ $60 \mathrm{mg} \mathrm{O}_{3} / \mathrm{L}$ ) resulted in $>98 \%$ removal of the UMFI cr for alginate $\pm \mathrm{Ca}^{+2}$ and humic acids. The higher cleaning efficiency of $\mathrm{O}_{3}$ is attributed to higher oxidation reactions between $\mathrm{O}_{3}$, free radicals and $\mathrm{NOM} . \mathrm{O}_{3}$ can react with humic acids and polysaccharides and breakup the aromatic rings and glycosidic linkages which transform NOM molecules into oxygenated functional groups (Van Geluwe et al., 2011; Song et al., 2010). The oxygenated functional groups are hydrophilic which have lower propensity for adsorption on membrane surface. In this study, $\mathrm{O}_{3}$ oxidized humic acids and alginate substances on the ceramic membrane surface and transformed the fouling layer into more hydrophilic and porous structure which enhanced their removals from the membrane surface (Zhang et al., 2013; Sartor et al., 2008). This is supported by Song et al. (2010) who reported an $\mathrm{O}_{3}$ dose of $0.50 \mathrm{mg} \mathrm{O} / \mathrm{mgC}$, applied for 10 minutes to surface water increased the hydrophilicity of NOM by $45 \%$ which reduced membrane fouling. Although the irreversible fouling masses of alginate $+\mathrm{Ca}^{+2}$, alginate $-\mathrm{Ca}^{+2}$, and humic acids on the membrane were different $\left(0.35,0.22,0.18 \mathrm{mgC} / \mathrm{cm}^{2}\right.$, respectively), $\mathrm{O}_{3} \mathrm{CIP}$ for 1 hour at $15^{\circ} \mathrm{C}$ demonstrated equivalent reductions of hydrophilic alginate and hydrophobic humic acids fouling. Moreover, the $\% \mathrm{UMFI}_{\text {cr }}$ obtained by $\mathrm{O}_{3} \mathrm{CIP}$ at a ratio of $0.50 \mathrm{mg} \mathrm{O}_{3} / \mathrm{mgC}$ are higher than those obtained by $\mathrm{NaOCl}$ or $\mathrm{NaOH}$ at $\mathrm{pH} 11$ and $12(\mathrm{p}<0.05)$ despite the difference in characteristics of the tested NOM foulants. A lower ozone ratio of $0.25 \mathrm{mg} \mathrm{O} / \mathrm{mg} \mathrm{C}$ was found to be less effective at cleaning the membrane. For this condition, the $\% \mathrm{UMFI}_{\mathrm{cr}}$ recovered decreased to $64 \%, 68 \%$ and $74 \%$ for alginate $+\mathrm{Ca}^{+2}$, alginate $-\mathrm{Ca}^{+2}$, and humic acids, respectively. 


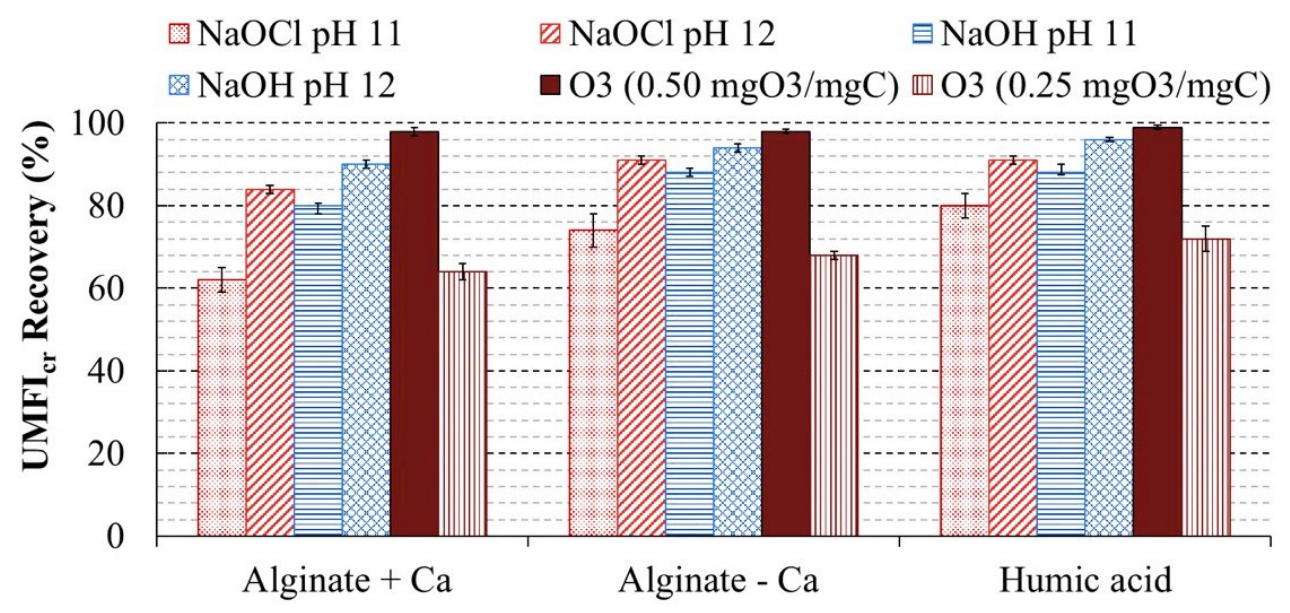

Figure 7.4- Effect of cleaning $\mathrm{pH}$ and cleaning agent on the removal of $\mathrm{UMFI}_{\mathrm{cr}}$ of ceramic UF*

*Cleaning time: $\mathrm{NaOCl}$ and $\mathrm{NaOH}$ CIP: 4 hrs; $\mathrm{O}_{3} \mathrm{CIP}: 1 \mathrm{hr}$.

* $\mathrm{UMFI}_{\text {cr }}$ : chemically reversible fouling index: ((UMFI $\left.\left.\mathrm{U}_{\text {hir }}-\mathrm{UMFI}_{\text {cir }}\right) / \mathrm{UMFI}_{\text {hir }}\right) \mathrm{x} 100$

While $\mathrm{O}_{3} \mathrm{CIP}$ at ratio of $0.50 \mathrm{mg} \mathrm{O} / \mathrm{mg} \mathrm{C}$ was effective for the removal of alginate $\pm \mathrm{Ca}^{+2}$ and humic acids, different $\mathrm{NOM}$ fractions responded differently to the $\mathrm{NaOCl}$ and $\mathrm{NaOH}$ cleaning (refer to Figure 7.4). $\mathrm{NaOCl}$ at $\mathrm{pH} 11$ or 12 showed an equivalent cleaning of alginate $-\mathrm{Ca}^{+2}$ and humic acids ( $\mathrm{p}>0.05$ ). The same can be said for $\mathrm{NaOH}$ at $\mathrm{pH} 11$ or 12 . On the other hand, in the case of alginate $+\mathrm{Ca}^{+2}$, the $\% \mathrm{UMFI}_{\mathrm{cr}}$ values recovered by $\mathrm{NaOCl}$ at $\mathrm{pH} 11$ and 12 were lower than those obtained with alginate $-\mathrm{Ca}^{+2}$ and humic acids. It is commonly known that $\mathrm{NaOCl}$ is important for cleaning organic substances (Shi et al., 2014; Porcelli et al., 2011; Arnal et al., 2009). It increases the repulsion between the negatively charged functional groups, which results in a stretched, linear configuration of NOM, thus loosening the fouling layer and enhancing the efficacy of cleaning (Shi et al., 2014). Nevertheless, the $\mathrm{NaOCl}$ cleaning did not prove to be efficient when alginate $+\mathrm{Ca}^{+2}$ was present; i.e., the $\% \mathrm{UMFI}_{\text {cr }}$ recovered for alginate $+\mathrm{Ca}^{+2}$ with $\mathrm{NaOCl}$ at $\mathrm{pH} 11$ and 12 were significantly lower than that for alginate $-\mathrm{Ca}^{+2}$ and humic acids $(\mathrm{p}<0.05)$. This could 
be attributed to the high irreversible mass of alginate $+\mathrm{Ca}^{+2}$ compared to alginate $-\mathrm{Ca}^{+2}$ and humic acids (refer to Table 7.1). While $\mathrm{NaOCl}$ is known to cause swelling to hydrophobic polymeric membranes, and this assists in flushing out foulant material within the pores (Wang et al., 2018; Zhang et al., 2017; Ferrer et al., 2016; Regula et al., 2014) the same cannot be said for ceramic membranes since they have solid structures and hydrophilic surfaces, thus, the swelling effect is not possible. Thus, in ceramic membrane the impact of $\mathrm{NaOCl}$ as a cleaning agent is reduced. Other factors such as the hydrophilic nature of the ceramic SiC membrane, and the surface tension $(\lambda)$ differences of the CIP waters may had an impact on the cleaning mechanisms.

Table 7.2 presents the contact angle and $\lambda$ values determined for $\mathrm{NaOCl}$ and $\mathrm{NaOH} \mathrm{CIP}$ waters. The $\lambda$ value for pure water was $70.8 \pm 0.5 \mathrm{mN} / \mathrm{m}$ at $35^{\circ} \mathrm{C}$. In the current literature, the mean values for water surface tension at $25^{\circ} \mathrm{C}$ range from $70.0 \mathrm{mN} / \mathrm{m}$ to $72.7 \mathrm{mN} / \mathrm{m}$ (Beattie et al., 2014; Estrela et al., 2005) agreeing with the value obtained in this study. It can be clearly seen that the addition of $\mathrm{NaOCl}$ and/or $\mathrm{NaOH}$ increased the $\lambda$ value of water (refer to Table 7.2). The $\lambda$ value of $\mathrm{NaOCl}$ CIP water at $\mathrm{pH} 11$ was $77.2 \pm 0.7 \mathrm{mN} / \mathrm{m}$ which had the highest measured contact angle of $58.7^{\circ}$. The contact angle and $\lambda$ measured for $\mathrm{NaOH}$ CIP at $\mathrm{pH} 11$ were $42.8^{\circ}$ and $73.1 \pm 0.1 \mathrm{mN} / \mathrm{m}$, respectively, which are much lower than the $\lambda$ values for $\mathrm{NaOCl} \mathrm{CIP} \mathrm{solution.} \mathrm{At} \mathrm{pH} 12$, the contact angle and $\lambda$ values for $\mathrm{NaOCl}$ decreased to $52.1^{\circ}$ and $74.1 \pm 0.5 \mathrm{mN} / \mathrm{m}$ whereas slight changes were noted in the contact angle and $\lambda$ values for $\mathrm{NaOH}$ at $\mathrm{pH} 12,41.6^{\circ}$ and $72.6 \pm 0.5 \mathrm{mN} / \mathrm{m}$ respectively. The results of our study agree with the results of Estrela et al. (2005) and Beattie et al. (2014) who obtained $75.0 \mathrm{mN} / \mathrm{m}$ for $\mathrm{NaOCl}$ and $72.8 \mathrm{mN} / \mathrm{m}$ for $\mathrm{NaOH}$, at $\mathrm{pH}$ of 12 respectively. 
These results indicate that for $\mathrm{NaOCl}$ at $\mathrm{pH} 11$, the high $\lambda$ value supressed the $\mathrm{NaOCl}$ cleaning efficiency compared to $\mathrm{NaOH}$ alone. $\mathrm{NaOH}$ appears to have a lower effect on surface tension compared to $\mathrm{NaOCl}$. This may explain why $\mathrm{NaOH}$ resulted in better fouling resistance recovery under the different $\mathrm{pH}$ conditions examined compared to $\mathrm{NaOCl}$.

Moreover, the increased cleaning efficiency observed with $\mathrm{NaOCl}$ at $\mathrm{pH} 12$ was related to the high concentration of $\mathrm{NaOH}$ in the wash water solution (i.e., more $\mathrm{NaOH}$ was added to bring the $\mathrm{pH}$ to 12 ) which enhanced the diffusion of chlorine at higher $\mathrm{pH}$ of 12 (Wang et al., 2018). In addition, Table 7.2 shows lower contact angle and the $\lambda$ values of the combined $\mathrm{NaOCl}$ and $\mathrm{NaOH}$ mixture, $41.7^{\circ}$ and $73.5 \pm 1.0 \mathrm{mN} / \mathrm{m}$ at $\mathrm{pH} 11$, and $41.5^{\circ}$ and $72.1 \pm 0.3 \mathrm{mN} / \mathrm{m}$ at $\mathrm{pH} 12$ compared to $\mathrm{NaOCl}$ or $\mathrm{NaOH}$ alone. The wetting ability of $\mathrm{NaOCl}$ and $\mathrm{NaOH}$ at $\mathrm{pH} 11$ can be enhanced with the addition of nonionic surfactants. For example, we tested the addition of $500 \mathrm{mg} / \mathrm{L}$ of Tween 80 which reduced the $\lambda$ values of $\mathrm{NaOCl}$ and $\mathrm{NaOH}$ solutions to $42.0 \pm 0.9 \mathrm{mN} / \mathrm{m}$ and $33.0 \pm 0.5 \mathrm{mN} / \mathrm{m}$, respectively.

Table 7.2- Average surface tension values of CIP water $(n=3)$

\begin{tabular}{ccc}
\hline CIP Condition & Contact angle & Surface Tension $(\mathrm{mN} / \mathrm{m})$ \\
\hline $\mathrm{NaOCl}$ pH 11 & $58.7^{0} \pm 3.3^{0}$ & $77.2 \pm 0.7$ \\
$\mathrm{NaOCl}$ pH 12 & $52.1^{0} \pm 1.8^{0}$ & $74.1 \pm 0.5$ \\
$\mathrm{NaOH} \mathrm{pH} \mathrm{11}$ & $42.8^{0} \pm 2.1^{0}$ & $73.1 \pm 0.1$ \\
$\mathrm{NaOH} \mathrm{pH} \mathrm{12}$ & $41.6^{0} \pm 3.6^{0}$ & $72.6 \pm 0.5$ \\
$\mathrm{NaOCl}+\mathrm{NaOH} \mathrm{pH} \mathrm{11}$ & $41.7^{0} \pm 1.4^{0}$ & $73.5 \pm 1.0$ \\
$\mathrm{NaOCl}+\mathrm{NaOH} \mathrm{pH} \mathrm{12}$ & $41.5^{0} \pm 2.5^{\circ}$ & $72.1 \pm 0.3$ \\
\hline
\end{tabular}


Figure 7.5 shows the \% carbon removed by chemical cleaning (i.e. carbon in the $\mathrm{NaOCl}$ $\mathrm{NaOH}$, and $\mathrm{O}_{3}$ wash waters) versus the resistance recovery (represented by $\% \mathrm{UMFI}_{\mathrm{cr}}$ ). It can be clearly seen that the carbon data correlates well with the fouling resistance removal $\left(\mathrm{R}^{2} \approx 0.86-0.92\right)$. On the other hand, $\mathrm{O}_{3} \mathrm{CIP}$ demonstrated higher removal of carbon compared to $\mathrm{NaOCl}$ or $\mathrm{NaOH}$. Since fouling irreversibility poses major implications during filtration with respect to membrane life time, the use of $\mathrm{O}_{3} \mathrm{CIP}$ could be more beneficial than $\mathrm{NaOCl}$ and $\mathrm{NaOH}$ for controlling irreversible fouling of $\mathrm{NOM}$ when utilizing ceramic membranes for drinking water treatment.
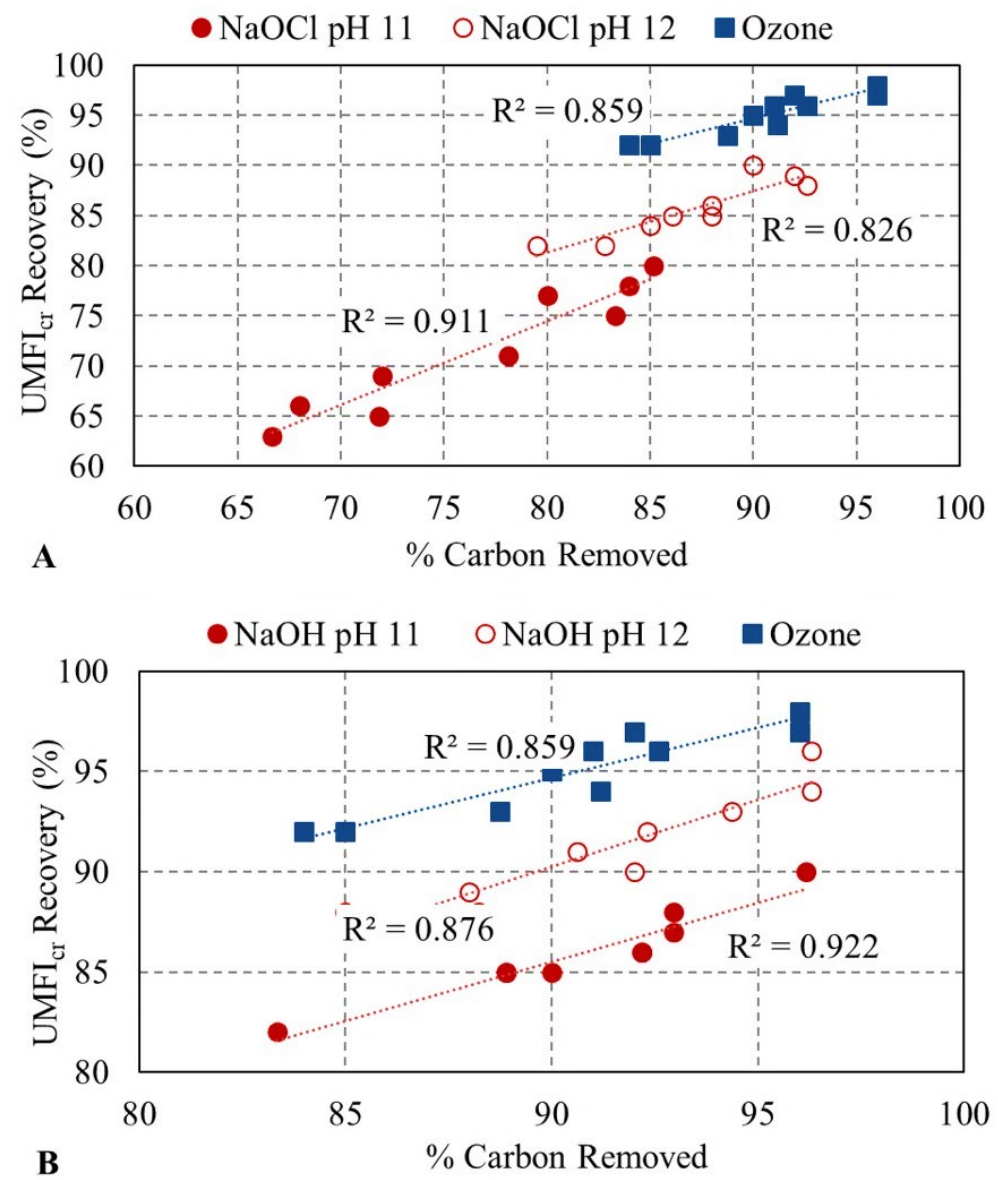

Figure 7.5- Correlations between chemically reversible fouling index recovery (UMFI $\mathrm{cr}_{\mathrm{rr}}$ and \% carbon removed by various chemical cleaning: (A) $\mathrm{NaOCl}$ CIP vs. $\mathrm{O}_{3} \mathrm{CIP}$; (B) $\mathrm{NaOH}$ CIP vs. $\mathrm{O}_{3}$ CIP. Ozone CIP at a ratio of $0.50 \mathrm{mg} \mathrm{O} / \mathrm{mg} \mathrm{C}$. 
Measurements were made during the $\mathrm{O}_{3} \mathrm{CIP}$ in order to define the optimal duration of cleaning (as shown in Figures 7.6A-B). It can be seen that as the cumulative $\mathrm{O}_{3}$ demand increased (Figure 7.6A), more carbon was removed for the different NOM types (Figure 7.6B). However, the carbon removal by $\mathrm{O}_{3}$ with time was dependent on the type of NOM on the membrane. For alginate $\pm \mathrm{Ca}^{+2}$, the cumulative carbon removed (Figure 7.6B) increased slowly within the first 30 minutes of cleaning (i.e. $55 \%$ of total irreversible carbon mass was removed) and then increased sharply afterwards. On the other hand, humic acid carbon removal trend (Figure 7.6B) showed faster increase within the first 30 minutes of cleaning (i.e. $78 \%$ of total irreversible carbon mass was removed) followed by a moderate increase in carbon removal. This demonstrates that oxidation of hydrophilic alginate by $\mathrm{O}_{3}$ was slower compared to hydrophobic NOM (i.e. humic acids). Moreover, this could be attributed to the differences in the irreversible carbon masses between the different NOM types. Overall, $\mathrm{O}_{3} \mathrm{CIP}$ cleaning using $0.50 \mathrm{mg} \mathrm{O} / \mathrm{mg} \mathrm{C}$, for 1 hour at 15 ${ }^{\circ} \mathrm{C}$, was effective in removing alginate and humic acids fouling. 


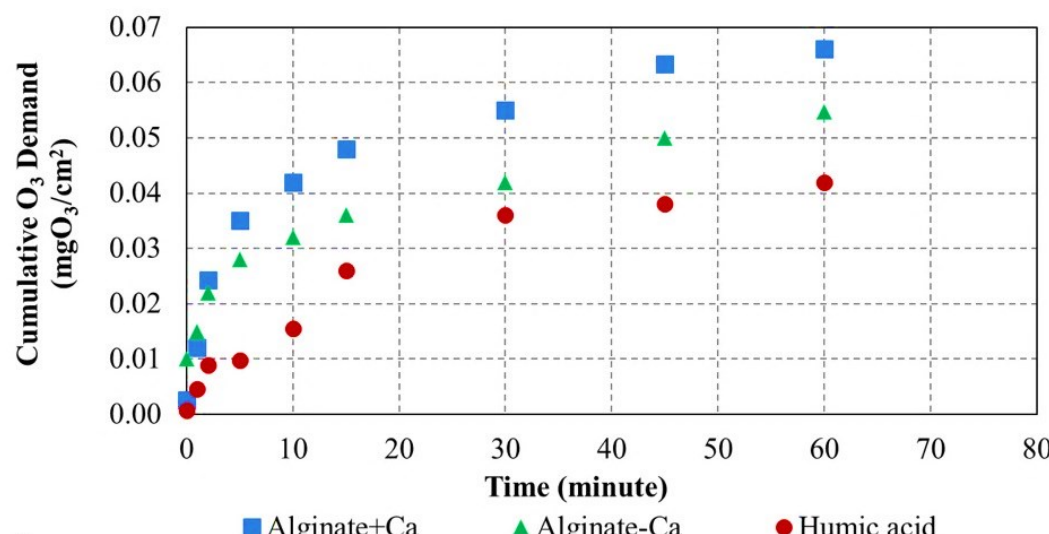

A

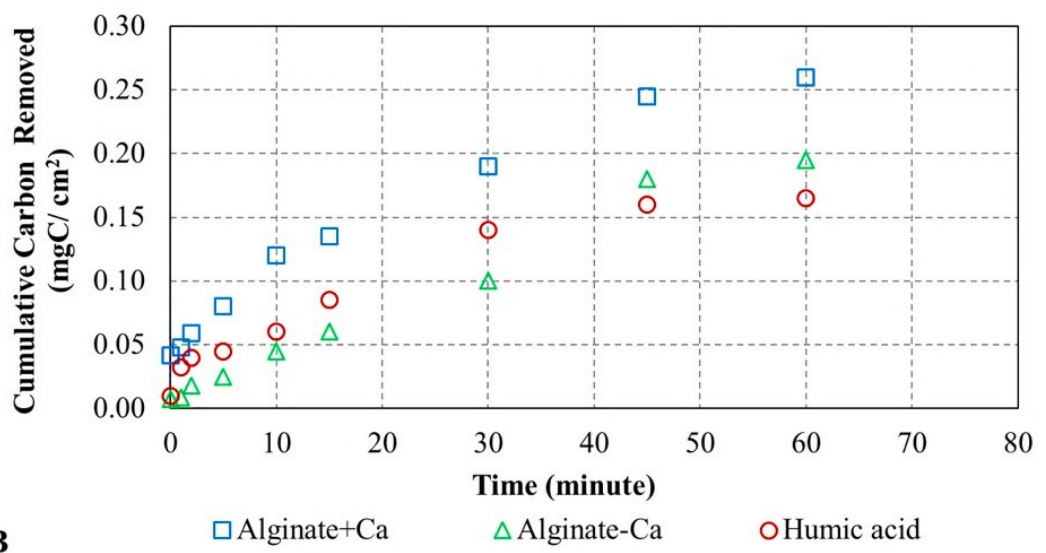

Figure 7.6- (A) Cumulative $\mathrm{O}_{3}$ demand versus cleaning time; (B) Cumulative carbon removed versus cleaning time. $\mathrm{O}_{3} \mathrm{CIP}$ at a ratio of $0.50 \mathrm{mg} \mathrm{O} / 3 \mathrm{mg} \mathrm{C}$

\subsubsection{Ceramic UF cleaning sequence: combined use of $\mathrm{NaOCl}$ and $\mathrm{NaOH}$ versus $\mathrm{O}_{3}$}

Irreversible membrane fouling is caused by the strong adsorption of NOM on the membrane surface or within its pores. The cleaning efficiency of NOM would varies depending on the characteristics of chemical reagent used for cleaning. The results in section 7.3.2 showed superior removal of alginate and humic acids foulants with $\mathrm{O}_{3}$ CIP and an improved cleaning at $\mathrm{pH}$ of 12 when utilizing a single step approach to chemical cleaning with the $\mathrm{NaOCl}$ or $\mathrm{NaOH}$ solutions. Ceramic membranes have high chemical resistance, and therefore, a consecutive or combined use of $\mathrm{NaOCl}$ and $\mathrm{NaOH}$ cleaning 
approach can be utilized which may be more effective than a two steps cleaning. However, to date there is little/no research in this area. Figure 7.7 shows that after $\mathrm{NaOCl} / \mathrm{NaOH}$ cleaning sequence, the overall $\% \mathrm{UMFI}_{\mathrm{cr}}$ were $92 \%, 94 \%$ and $94 \%$ for alginate $+\mathrm{Ca}^{+2}$, alginate $-\mathrm{Ca}^{+2}$, and humic acid, respectively. The cleaning efficiency using sequential $\mathrm{NaOCl} / \mathrm{NaOH}$ is equivalent to that using $\mathrm{NaOH}$ alone at $\mathrm{pH} 12(\mathrm{p}>0.05)$. The cleaning efficiency increased significantly $(\mathrm{p}<0.05)$ when $\mathrm{NaOH}$ was used before $\mathrm{NaOCl}$ (i.e., $\mathrm{NaOH} / \mathrm{NaOCl}$ sequence), and resulted in $\% \mathrm{UMFI}_{\text {cr }}$ of $98 \%, 99 \%$, and $99 \%$ for alginate + $\mathrm{Ca}^{+2}$, alginate $-\mathrm{Ca}^{+2}$, and humic acid respectively. $\mathrm{NaOH}$ is known to increase the solubility of humic acid and polysaccharide substances by hydrolysis and solubilization, which can change the configuration of NOM and make the fouling layer into a looser and more open structure (Shi et al., 2014). This could provide an easier path for $\mathrm{NaOCl}$ reaching the foulant layer and oxidizing NOM. Similar results were reported by Kimura et al. (2004) study in which the flux recovery of a polymeric membrane fouled by NOM improved with cleaning using $\mathrm{NaOH} / \mathrm{NaOCl}$ sequence compared to $\mathrm{NaOH}$ alone.

The combined use of $\mathrm{NaOCl}$ and $\mathrm{NaOH}$ refer to Figure 7.7, resulted in $\% \mathrm{UMFI}_{\mathrm{cr}}$ of $>98 \%$ for alginate $\pm \mathrm{Ca}^{+2}$, and humic acids. It is postulated that the $\mathrm{NaOH}$ provided an easier path for $\mathrm{NaOCl}$ to reach the foulant layer and oxidize NOM (Liang et al., 2008; Strugholtz et al., 2005). A previous study found that $\mathrm{NaOH}$ improved the capacity of $\mathrm{H}_{2} \mathrm{O}_{2}$ to remove and oxidize foulants similar to this work (Strugholtz et al., 2005). In addition, modifying surface tension while maintain $\mathrm{pH}$ has been demonstrated by others to improve cleaning (Wang et al., 2018; Ujihara et al., 2016; Levitsky et al., 2012). Thus, the lower surface tension of the combined $\mathrm{NaOCl}$ and $\mathrm{NaOH} \mathrm{CIP}$ water at $\mathrm{pH} 12,72.1 \pm 0.3 \mathrm{mN} / \mathrm{m}$, (refer to 
Table 7.2), may have improved the wetting ability of the CIP water and thus resulted in a higher cleaning efficiency. Therefore, not only the oxidation reactions, but also the mass transfer of chemicals and detached foulants need to be considered for efficient cleaning (Liu et al., 2001). The $\% \mathrm{UMFI}_{\mathrm{cr}}$ recovered by $\mathrm{O}_{3} \mathrm{CIP}$ at a ratio of $0.50 \mathrm{mgO} / \mathrm{mgC}$ are similar to $\% \mathrm{UMFI}_{\mathrm{cr}}$ recovered by the $\mathrm{NaOH} / \mathrm{NaOCl}$ cleaning sequence and combined $\mathrm{NaOCl}+\mathrm{NaOH}$ CIP $(\mathrm{p}>0.05)$.
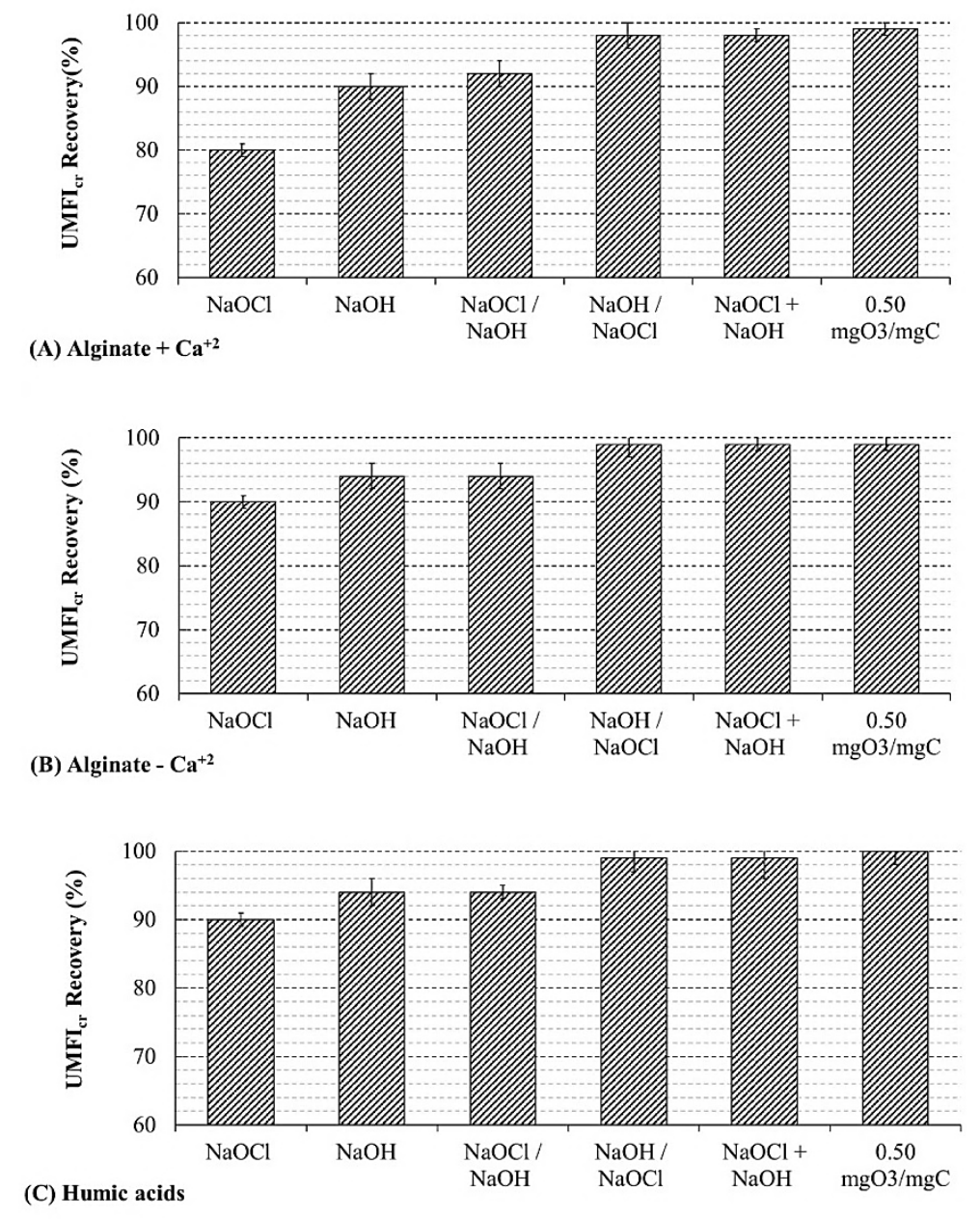

Figure 7.7- Recovery of UMFIcr following various ceramic UF cleaning sequence*. (A) Alginate $+\mathrm{Ca}^{+2}$; (B) Alginate- $\mathrm{Ca}^{+2}$ (C) Humic acids.

* $\mathrm{NaOCl}$ and $\mathrm{NaOH}$ CIP: $\mathrm{NaOCl} 500 \mathrm{mg} \mathrm{Cl} / \mathrm{L} ; \mathrm{NaOH} 0.01 \mathrm{~N}$, cleaning pH 12; cleaning time 4 hrs (total). $\mathrm{NaOCl} / \mathrm{NaOH}: \mathrm{NaOCl}$ followed by $\mathrm{NaOH} ; \mathrm{NaOCl}+\mathrm{NaOH}: \mathrm{NaOCl}$ mixed with $\mathrm{NaOH}$. Ozone CIP: $0.50 \mathrm{mgO}_{3} / \mathrm{mgC}$; cleaning time $1 \mathrm{hr}$. UMFI $\mathrm{cr}$ : chemically reversible fouling index. 
Table 7.3 presents cost comparisons of $\mathrm{NaOCl}, \mathrm{NaOH}$, and $\mathrm{O}_{3} \mathrm{CIP}$. It can be clearly seen that based on the amount of chemicals used in this study, $\mathrm{NaOCl}$ has the highest cost $(0.005$ US\$ per clean) followed by $\mathrm{NaOH}\left(0.002\right.$ US\$ per clean) and $\mathrm{O}_{3}(0.001$ US\$ per clean). Although $\mathrm{O}_{3}$ investment costs were not considered, the amount of $\mathrm{O}_{3}$ used in this study indicate that $\mathrm{O}_{3}$ is the least expensive chemical compared to $\mathrm{NaOCl}$ and $\mathrm{NaOH}$. Furthermore, $\mathrm{O}_{3}$ CIP step requires a short cleaning duration (i.e., 1 hour), thus, less cleaning time compared to $\mathrm{NaOCl}$ and/or $\mathrm{NaOH}$ CIP (optimal cleaning time of 4 hours). These advantages suggest that the use of $\mathrm{O}_{3} \mathrm{CIP}$ with ceramic membranes provides an effective and economical cleaning option over conventional cleaning chemicals. Future investigations are recommended to determine the long-term capital/investment costs of $\mathrm{O}_{3}$ CIP with ceramic membrane systems.

Table 7.3- Cost comparisons of $\mathrm{NaOCl}, \mathrm{NaOH}$, and $\mathrm{O}_{3}$ (for one clean of the lab module)

\begin{tabular}{|c|c|c|c|}
\hline & $\mathrm{NaOCl}$ (pH 12) & NaOH (pH 12) & $\mathbf{O}_{3}$ \\
\hline Concentration & $\begin{array}{l}500 \mathrm{mg} \mathrm{Cl} / \mathrm{L}+ \\
60 \mathrm{mg} / \mathrm{L} \mathrm{NaOH}\end{array}$ & $400 \mathrm{mg} / \mathrm{L}$ & $60 \mathrm{mg} / \mathrm{L}$ \\
\hline $\begin{array}{c}\text { Cleaning Water } \\
\text { Volume }\end{array}$ & $4 \mathrm{~L}$ & $4 \mathrm{~L}$ & $4 \mathrm{~L}$ \\
\hline Chemical Amount & $\begin{array}{l}2000 \mathrm{mg} \mathrm{Cl}{ }_{2}+ \\
240 \mathrm{mg} \mathrm{NaOH}\end{array}$ & $1600 \mathrm{mg}$ & $240 \mathrm{mg}$ \\
\hline Cleaning Time & $4 \mathrm{hrs}$ & $4 \mathrm{hrs}$ & $1 \mathrm{hr}$ \\
\hline Cost $/ \mathbf{k g}$ & $\begin{array}{l}\text { USD } 2.3 / \mathrm{kg} \mathrm{Cl}_{2}{ }^{*}+ \\
\text { USD } 1.2 / \mathrm{kg} \mathrm{NaOH}\end{array}$ & USD $1.2 / \mathrm{kg}$ & USD $2.52 / \mathrm{kg} \mathrm{O}_{3}{ }^{* *}$ \\
\hline Cost / Clean & USD 0.005 & USD 0.002 & USD 0.001 \\
\hline
\end{tabular}




\subsection{Conclusions}

This research examined the utilization of $\mathrm{O}_{3}$ CIP with ceramic UF membranes for controlling irreversible fouling of hydrophilic (alginate $\pm \mathrm{Ca}^{+2}$ ) and hydrophobic (humic acids) NOM compared to conventional cleaning chemicals ( $\mathrm{NaOCl}$ and $\mathrm{NaOH})$. Key points from the research are summarized here:

- $\mathrm{O}_{3}(0.50 \mathrm{mgO} / \mathrm{mgC})$ was equally effective at removing alginate $\pm \mathrm{Ca}^{+2}$ and humic acids NOM foulants, while both $\mathrm{NaOH}$ and $\mathrm{NaOCl}$ were less effective with the alginate + $\mathrm{Ca}^{+2}$ condition, indicating a higher sensitivity of traditional cleaning chemical to water quality conditions for chemical cleaning and a more robust cleaning with $\mathrm{O}_{3}$.

- For the conditions tested, a 1 hour of $\mathrm{O}_{3} \mathrm{CIP}$ at a ratio of $0.50 \mathrm{mgO}_{3} / \mathrm{mgC}$ demonstrated an equivalent performance to 4 hours of cleaning using sequential $\mathrm{NaOH} / \mathrm{NaOCl}$ or combined $\mathrm{NaOCl}+\mathrm{NaOH}$ CIP $\left(\% \mathrm{UMFI}_{\mathrm{cr}}>98 \%\right)$. Future investigations should assess the optimal ozonation conditions (time vs. $\mathrm{O}_{3}$ concentration) required to maximize $\mathrm{O}_{3}$ CIP performance while minimizing costs.

- Cleaning efficiency of $\mathrm{NaOCl}$ and $\mathrm{NaOH}$ is governed by the $\mathrm{pH}$ of the CIP water. Future research should examine the effectiveness of surfactant addition versus $\mathrm{pH}$ adjustment for more economical and effective control of irreversible fouling of ceramic membranes.

\subsection{Acknowledgements}

The authors would like to acknowledge the Saudi Arabia Ministry of Education (MOE) and Natural Sciences and Engineering Research Council of Canada (NSERC) for helping 
fund this research. The authors wish to thank the CREDEAU laboratories at Polytechnique Montréal for providing the facilities to conduct this work as well as the collaboration of Mireille Blais. We also wish to thank Geoff Seatter and Kerwin Lewis for their help with installation of the automated membrane filtration system.

\subsection{References}

Arkhangelsky, E., Kuzmenko, D., Gitis, V., 2007. Impact of chemical cleaning on properties and functioning of polyethersulfone membranes. Journal of Membrane Science, 305, 176-184.

Arnal, J.M., Garcia-Fayos, B., Sancho, G., Verdu, M., 2009. Ultrafiltration membrane cleaning with different chemical solutions after treating surface water, Desalination and Water Treatment 7, 198-205.

Bader, H., Hoigin, J., 1981. Determination of ozone in water by the indigo method. Water Research 449, 456.

Beattie, J., Djerdjev, A., Weale, A., Kallay, N., Lutzenkirchen, J., Preocanin, T., Selmani A., 2014. $\mathrm{pH}$ and the surface tension of water. Journal of Colloid and Interface Science $422,54-57$. 
Estrela, C., Estrela, C., Guimaraes, L., Silva, R., Pecora, J., 2005. Surface tension of calcium hydroxide associated with different substances. Journal of Applied Science 13(2): $152-6$.

Ferrer, O., Lefevre, B., Parts, G., Bernat, X., Gibret, O., Miquel, P., 2016. Reversibility of fouling on ultrafiltration membrane by backwashing and chemical cleaning: differences in organic fractions behaviour. Desalination and Water Treatment 57, 8593-8607.

Hashino, M., Hirami, K., Katagiri, K., Kubota, T., 2011. Effects of three natural organic matter types on cellulose acetate butyrate microfiltration membrane fouling. Journal of Membrane Science 379, 233- 238.

Hilal, N., Oluwaseun, O., Miles, N., Nigmatullin, R., 2005. Methods employed for control of fouling in MF and UF membranes: A Comprehensive Review. Separation Science and Technology, 40, 1957-2005.

Huang, H., Young, T., Jacangelo, J., 2008. Unified membrane fouling index for low pressure membrane filtration of natural waters: principles and methodology. Environmental Science Technology 42, 714-720.

Jamal, S., Chang, S., Zhou, H., 2014. Filtration behavior and mechanisms of polysaccharides. Membranes 4, 319-332. 
Karnik, B., Davies, S., Chen, K.C., Jaglowski, D.R., Baumann, M.J., Masten, S.J., 2005. Effects of ozonation on the permeate flux of nano crystalline ceramic membranes. Water Research 39, 728-734.

Katsoufidou, K., Sioutopoulos, D., Yiantsios, S., Karabelas, A., 2010. UF membrane fouling by mixtures of humic acids and sodium alginate: Fouling mechanisms and reversibility. Desalination 264, 220-227.

Kim, J., Davies, S., Baumann, M., Tarabara, V., Masten, S., 2008. Effect of ozone dosage and hydrodynamic conditions on the permeate flux in a hybrid ozonation-ceramic ultrafiltration system treating natural waters. Journal of Membrane Science 311, 165- 172.

Kimura, K., Hane, Y., Watanabe, Y., Amy, G., Ohkuma, N., 2004. Irreversible membrane fouling during ultrafiltration of surface. Water Research 38, 3431-3441.

Lee, S., Dilaver, M., Park, P., Kim, J., 2013. Comparative analysis of fouling characteristics of ceramic and polymeric microfiltration membranes using filtration models. Journal of Membrane Science 432, 97-105.

Levitsky, I., Duek, A., Naim, R., Arkhangelsky, E., Gitis, V., 2012. Cleaning UF membranes with simple and formulated solutions. Chemical Engineering Science 69, 679683. 
Liang, H., Gong, W., Chen, J., Li, G., 2008. Cleaning of fouled ultrafiltration (UF) membrane by algae during reservoir water treatment. Desalination 220, 267-272.

Liu, C., Caothien, S., Hayes, J., Caothuy, T., Otoyo, T. and Ogawa, T., 2001. Membrane chemical cleaning: from art to science. Process membrane technology conference Port Washington, NY: 1-25.

Porcelli, N., Judd, S., 2011. Chemical cleaning of potable water membranes: A review. Separation and Purification Technology 71, 137-143.

Puspitasari, V., Granville, A., Le-Clech, P., Chena, V., 2010. Cleaning and ageing effect of sodium hypochlorite on polyvinylidene fluoride (PVDF) membrane. Separation and Purification Technology, 72, 301-308.

Regula, C., Carretier, E., Wyart, Y., Gesan-Guiziou, G., Vincent, A., Boudot, D., Moulin, P., 2014. Chemical cleaning/disinfection and ageing of organic UF membranes: A review. Water Research 56, 325-365.

Sartor, M., Gatjal, H., Mavrov, V., 2008. Demonstration of a new hybrid process for the decentralised drinking and service water production from surface water in Thailand. Desalination 222, 528-540. 
Shi, X., Tal, G., Hankins, N.P., Gitis, V., 2014. Fouling and cleaning of ultrafiltration membranes: a review. Journal of Water Process Engineering 1, 121-138.

Song, Y., Dong, B., Gao, N., Xia, S., 2010. Huangpu River water treatment by microfiltration with ozone pretreatment. Desalination 250, 71-75.

Strugholtz, S., Sundaramoorthy, K., Panglisch, S., Lerch, A. BriJggef, A., Gimbe, R., 2005. Evaluation of the performance of different chemicals for cleaning capillary membranes. Desalination 179, 191-202.

Ujihara, R., Mino, Y., Takahashi, T., Shimizu, Y., Matasuyama, H., 2016. Effects of the ionic strength of sodium hypochlorite solution on membrane cleaning. Journal of Membrane Science 514, 566-573.

Van Geluwe, S., Braeken, L., Van der Bruggen, B., 2011. Ozone oxidation for the alleviation of membrane fouling by natural organic matter: A review. Water Research 45, $3551-3570$.

Wang, Q., Zeng H., Wua, Z., Cao, J., 2018. Impact of sodium hypochlorite cleaning on the surface properties and performance of PVDF membranes. Applied Surface Science 428, 289-295. 
Wang, X., Ma, J., Wang, Z., Chen, H., Liu, M., Wu, Z., 2018. Re-investigation of membrane cleaning mechanisms using $\mathrm{NaOCl}$ : Role of reagent diffusion. Journal of Membrane Science 550, 278-285.

Wang, Z., Jinxing M., Chuyang, T., Katsuki, K., Wang, Q., Han., X., 2014. Membrane cleaning in membrane bioreactors: A review. Journal of Membrane Science 468, 276-307.

Xiao, K., Wang, X., Huang, X., Waite, T., Wen, X., 2009. Analysis of polysaccharide, protein and humic acid retention by microfiltration membranes using Thomas' dynamic adsorption model. Journal of Membrane Science 342, 22-34.

Zhang, X., Guo, J., Wang, L., Hu, J., Zhu, J., 2013. In situ ozonation to control ceramic membrane fouling in drinking water treatment. Desalination 328, 1-7.

Zhang, Y., Wang, J., Geo, F., Chen, Y., Zhang, H., 2017. A comparison study: The different impacts of sodium hypochlorite on PVDF and PSF ultrafiltration (UF) membranes. Water Research 109, 227-236. 


\section{Chapter 8}

\section{Summary, Conclusions and Recommendations}

This chapter presents a summary of findings and conclusions from individual chapters followed by overall significant conclusions and contributions, and recommendations for future membrane work for drinking water treatment.

\subsection{Summary of findings and conclusions}

Stage 1: The applicability of the MFI-UF to predict fouling potential of different NOM fractions under changes in pressure and water temperature conditions was examined (Chapter 3). The following conclusions were made:

- The MFI-UF exp method was sensitive to the type of NOM in feed water. A mixture of humic acid, BSA, and alginate resulted in the highest MFI-UF value compared to individual NOM solutions highlighting the need to identify specific NOM fractions in water to better understand potential fouling changes.

- All NOM types exhibited higher fouling propensity as water temperature decreased and pressure increased indicating the importance of assessing NOM fouling at expected conditions versus at standardized conditions alone. The developed MFI-UF normalization model (MFI-UF nor) was useful in estimating the fouling potential away from standard testing conditions. Therefore, MFI-UF nor model can be used to determine potential changes in NOM fouling behavior under changes in pressure and water temperature conditions. 
Stage 2: Building upon stage 1, the impact of water temperature variation on irreversible NOM fouling of a submerged polymeric UF membrane was examined using the MFI-UF and UMFI fouling index methods (Chapter 4). The changes in backwash and cleaning effectiveness with temperature were investigated. The following conclusions were made:

- Water temperature demonstrated an impact on fouling and subsequent cleaning requirements for the submerged polymeric membrane. Irreversible fouling increased as temperature decreased from 35 to $5{ }^{\circ} \mathrm{C}$. As a results, backwash and chemical cleaning were less effective at lower temperature.

- Fouling and cleaning of the NOM mixture, BSA, and alginate solutions were sensitive to changes in water temperature whereas humic acid was least sensitive highlighting the need to identify specific NOM fractions in water to better understand potential changes in fouling and cleaning requirements.

- The MFI-UF fouling prediction demonstrated a useful fit with UMFI indices for establishing NOM irreversible fouling, highlighting an advantage of the MFI-UF as a robust fouling prediction index for low pressure polymeric membranes.

Stage 3: Building upon stages 1 and 2 of this research, the impact of water temperature variation on irreversible NOM fouling of a tubular ceramic UF membrane was examined using the MFI-UF and UMFI fouling index methods (Chapter 5). The changes in backwash and cleaning effectiveness with temperature were investigated. The following conclusions were made:

- The influence of feed water temperature on fouling of ceramic UF membranes is over and beyond simple water viscosity changes. The $\mathrm{UMFI}_{\text {nor }}$ values (i.e. after correcting 
for viscosity) showed that at $5{ }^{\circ} \mathrm{C}$, only $43 \%$ of the increased in fouling was attributed to the high viscosity of water whereas $57 \%$ was due to fouling. Also, at $35^{\circ} \mathrm{C}, 64 \%$ of the decreased in fouling was attributed to the low viscosity of water whereas $36 \%$ was due to fouling. Thus, fouling analysis performed at standard temperature $\left(20^{\circ} \mathrm{C}\right)$ will underestimate the impact of cold water condition on ceramic membranes performance.

- The irreversible fouling ratios increased at colder temperature $\left(5^{\circ} \mathrm{C}\right)$, along with decreased in backwash and chemical cleaning effectiveness. Backwash and chemical cleaning were equally effective at 20 and $35^{\circ} \mathrm{C}$ compared to $5{ }^{\circ} \mathrm{C}$. Thus, modification of chemical cleaning procedure at cold water temperature condition is necessary to control irreversible fouling and recover membrane permeability.

- MFI-UF fouling indices and NOM retention were in agreement with the UMFI values and NOM retention data obtained in the ceramic UF system. Thus, the utilization of the MFI-UF is useful for assessing the potential changes in NOM fouling behavior with temperature for ceramic membrane systems. Moreover, the MFI-UF offers simple and short filtration test that can be used as a rapid assessment of NOM fouling compared to the UMFI testing which is normally performed on long-term filtration data.

Stage 4: Fouling and cleaning behaviors of different model NOM solutions were comparatively assessed for polymeric and ceramic UF membranes (Chapter 6). The following conclusions were made:

- The NOM fouling order was similar for both membranes which was greatest for the NOM mixture and BSA followed by alginate $\left( \pm \mathrm{Ca}^{+2}\right)$ and lastly humic acid, indicating that both membranes are most susceptible to fouling by proteins. 
- Hydraulically irreversible fouling resistances of different NOM components were much higher for the polymeric UF compared to the ceramic UF highlighting an advantage of ceramic membranes with regards to physical cleaning compared to polymeric membranes.

- Stage 5: Builds on stage 4 to investigate the utilization of $\mathrm{O}_{3} \mathrm{CIP}$ with ceramic UF membranes for controlling irreversible fouling of hydrophilic and hydrophobic NOM compared to conventional cleaning chemicals $(\mathrm{NaOCl}$ and $\mathrm{NaOH})$. The following conclusions were made:

- $\mathrm{O}_{3} \mathrm{CIP}$ at a ratio of $0.50 \mathrm{mgO}_{3} / \mathrm{mgC}$ was effective in removing alginate $\pm \mathrm{Ca}^{+2}$ and humic acids NOM foulants compared to single cleaning using $\mathrm{NaOCl}$ or $\mathrm{NaOH} \mathrm{CIP}$, highlighting an advantage of $\mathrm{O}_{3}$ CIP with ceramic membranes for irreversible NOM fouling control.

- From the results achieved, a 1 hour of $\mathrm{O}_{3} \mathrm{CIP}$ at a ratio of $0.50 \mathrm{mgO}_{3} / \mathrm{mgC}$ demonstrated an equivalent performance to 4 hours of cleaning using combined $\mathrm{NaOCl}$ and $\mathrm{NaOH} \mathrm{CIP}$.

\subsection{Overall significant conclusions and contributions}

Significant conclusions were made in the course of this research. The key contributions of this research are:

- Since it is essential to reduce membrane fouling by NOM, implementing the MFI-UF testing using a $13 \mathrm{kDa}$ hollow fiber polymeric membrane is useful for assessing the fouling potential of different NOM feed waters under changes in water temperature 
conditions. A plant could perform the MFI-UF at standard temperature condition (i.e. $20{ }^{\circ} \mathrm{C}$ ) and then use the normalized model used in this research to adjust for water temperature changes to estimate the expected fouling in an operating polymeric and ceramic membrane systems.

- Changes in NOM fouling mechanisms for polymeric and ceramic membrane systems with temperature is over and beyond simple water viscosity changes. Irreversible NOM fouling is dominant at cold water condition, and therefore, utilizing the MFI-UF testing, implementing proper membrane pretreatment strategies and/or modifying cleaning procedures to reduce irreversible fouling of constituents having characteristics similar to that of humics, proteins, and polysaccharides are essential for long-term operational sustainability. Proteins particularly seem to be especially problematic for both polymeric and ceramic membranes.

- Although ceramic membranes are similar to their polymeric counterparts with regard to NOM fouling order, the robustness of ceramic membranes in terms of physical cleaning, highlight the ease of integration of ceramic membranes into drinking water applications.

- $\mathrm{O}_{3}$ CIP proved effective for reducing humics and polysaccharides irreversible fouling compared to conventional cleaning chemicals. However, studies are required to determine the optimal ozonation conditions (time vs. $\mathrm{O}_{3}$ concentration) for different types of water to maximize $\mathrm{O}_{3}$ CIP performance while minimizing costs. Since the ceramic membrane is quite robust, it allows for some creativity regarding fouling mitigation techniques, particularly backwashes and chemical cleaning. Therefore, this opens the door to some more unique future investigations. 


\subsection{Recommendations for future work}

Several results along the course of this research clearly indicate that further work on membrane fouling and cleaning is still needed. The MFI-UF method is useful to predict the fouling potential of various NOM feed waters under changing water temperature conditions with both polymeric and ceramic membrane systems. However, the MFI-UF, utilizing a hollow fiber membrane, is currently limited to academic lab based research and designing a commercial portable equipment of MFI-UF tester is important to facilitate the integration of the MFI-UF testing with polymeric and ceramic membrane systems.

Also, this research clearly demonstrated that membrane fouling can be underestimated at colder temperature. Northern areas, such as northern US and Canada, are the most sensitive to the effects of temperature change as water temperature can go below $5{ }^{\circ} \mathrm{C}$ in the winter and above $30{ }^{\circ} \mathrm{C}$ in the summer. Future studies should investigate the impacts of water temperature with actual source waters to better elucidate the complexities of real water systems and membrane fouling at low temperatures.

The ability of ceramic membranes to tolerate higher pressures and more challenging water qualities compared to polymeric membranes makes them ideal candidates for drinking water treatment. Ultimately, cost will always be a driving factor for membrane systems. Despite estimations that suggest that ceramic membranes are becoming cost competitive with polymeric membranes, the short-term, higher capital costs will often be an obstacle for ceramic membranes. However, with the higher costs of ceramic membranes does come higher quality and robustness. From the results achieved, under equivalent operational 
conditions, ceramic membranes can be physically and chemically cleaned more easily compared to polymeric membranes. In addition, the robustness of ceramic membranes against rigorous chemical cleaning conditions (i.e. ozone) provides flexibility of the system. Future research should examine different cleaning techniques for irreversible fouling control with ceramic membranes. A creative investigation of different backwash and chemical cleaning procedures could be beneficial for sustainable membrane operation. 


\section{$\underline{\text { References }}$}

Alhadidi, A., Blankert, B., Kemperman, A.J.B., Schippers, J.C., Wessling, M., Van der Meer, W.G.J., 2011. Effect of testing conditions and filtration mechanisms on SDI, Journal of Membrane Science 381, 142-151.

Alhadidi, A., Kemperman, A.J.B., Schippers, J.C., Wessling, M., Van der Meer, W.G.J., 2011. The influence of membrane properties on the silt density index. Journal of Membrane Science 384, 205-218.

Ando, M., Ishihara, S., Iwahori H., Tada, N., 2003. Peculiar or unexpected behavior of silt density index of pretreated water for desalination.

Ang, W., Lee, S., Elimelech, M., 2006. Chemical and physical aspects of cleaning of organic-fouled reverse osmosis membranes. Journal of Membrane Science 272, 198210.

Arkhangelsky, E., Kuzmenko, D., Gitis, V., 2007. Impact of chemical cleaning on properties and functioning of polyethersulfone membranes. Journal of Membrane Science 305, 176-184.

ASTM International, Designation: D8002 - 15 (Reapproved 2016). Standard test method for modified fouling index (MFI-0.45) of Water. 
ASTM International, Designation: D4189 - 07 (Reapproved 2014). Standard test method for silt density index (SDI) of water.

Baghoth, S.A., Sharma, S.K., Amy, G.L., 2011. Tracking natural organic matter (NOM) in a drinking water treatment plant using fluorescence excitation-emission matrices and PARAFAC. Water Research 45, 797.

Betancourt, W., Rose, J., 2004. Drinking water treatment processes for removal of Cryptosporidium and Giardia. Veterinary Parasitology 126, 219-234.

Bieroza, M., Baker, A., Bridgeman, J., 2010. Fluorescence spectroscopy as a tool for determination of organic matter removal efficiency at water treatment works. Drinking Water Engineering Science 3, 63.

Bieroza, M., Baker, A., Bridgeman, J., 2011. Assessment of low pH coagulation performance using fluorescence spectroscopy. Journal of Environmental Engineering 137, 596.

Blankert, B., Betlem, B.H.L., and Roffel, B., 2006. Dynamic optimization of a deadend filtration trajectory: Blocking filtration laws. Journal of Membrane Science 285, $90-95$. 
Boerlage, S., Kennedy, M., Aniye, M., Abogrean, E., Tarawneh, Z., Schippers, J., 2003. The MFI-UF as a water quality test and monitor. Journal of Membrane Science 211, $271-289$.

Boerlage, S., Kennedy, M., Dickson, M., El-Hodali, D., Schippers, J., 2002. The modified fouling index using ultrafiltration membranes (MFI-UF): characterisation, filtration mechanisms and proposed reference membrane. Journal of Membrane Science 197, 1-21.

Boerlage, S., Kennedy, M., Tarawneh, Z., De Faber, R., Schippers, J., 2004. Development of the MFI-UF in constant flux filtration. Desalination 161,103-1 13.

Burashid, K. Hussain, A.R, 2004. Seawater RO plant operation and maintenance experience: Addur desalination plant operation assessment. Desalination 165, (1-3): p. $11-22$.

Chang, E., Yang, S., Huang, C., Liang, C., Chiang, P., 2011. Assessing the fouling mechanisms of high-pressure nanofiltration membrane using the modified Hermia model and the resistance-in-series model. Separation and Purification Technology 79, $329-336$.

Crittenden, J., Trussell R., Hand D., Howe, K., Tchobanoglous, G., 2005. Water Treatment: Principles and Design, 2nd edition, Ed, Wiley, New Jersey, USA. 
Cui, Z., Peng, W., Fan, Y., 2013. Ceramic membrane filtration as seawater RO pretreatment: influencing factors on the ceramic membrane flux and quality. Desalination and Water Treatment 51, 2575-2583.

Dramas, L., Croue, J., 2013. Ceramic membrane as a pretreatment for reverse osmosis: interaction between marine organic matter and metal oxides. Desalination and Water Treatment 51, 1781-1789.

Fan, X., Tao, Y., Wang, L., Zhang, X., 2014. Performance of an integrated process combining ozonation with ceramic membrane ultra-filtration for advanced treatment of drinking water. Desalination 335, 47-54.

Farahbakhsh, K., Smith, D., 2006. Membrane filtration for cold regions - impact of cold water on membrane integrity testing. Journal of Environmental Engineering and Science 5, 69-75.

Fiksdal, L., Leiknes, T., 2006. The effect of coagulation with MF/UF membrane filtration for the removal of virus in drinking water. Journal of Membrane Science 279, $364-371$.

Freeman, S., Shorney-Darby, H., 2011. What's the buzz about ceramic membranes? Journal of AWWA 103, 12-13. 
Gone, D.L., Seidel, J.L., Batiot, C., Bamory, K., Ligban, R., Biemi, J., 2009. Using fluorescence spectroscopy EEM to evaluate the efficiency of organic matter removal during coagulation-flocculation of a tropical surface water (Agbo reservoir). Journals of Hazardous Materials 172, 693.

Guo, J., Wang, L., Zhu, J., 2013. Highly integrated hybrid process with ceramic ultrafiltration-membrane for advanced treatment of drinking water: A pilot study. Journal of Environmental Science and Health Part A 48, 1413-1419.

Guo, W., Ngo, H., Li, J., 2012. A mini-review on membrane fouling. Bioresource Technology 122, 27-34.

Habib, M., Unsia, H., Memon, A., Amin, U., Karim, Z., Khan A., Naveed, S., Ali, S, 2013. Predicting colloidal fouling of tap water by silt density index (SDI): Pore blocking in a membrane process. Journal of Environmental Chemical Engineering 1, $33-37$.

Hamad, J., Ha, C., Kennedy, M., Amy, G., 2013. Application of ceramic membranes for seawater reverse osmosis (SWRO) pre-treatment. Desalination and Water Treatment 51, 4881-489.

Hao R., Ren H., Li, J., Ma, Z., Wan H., Zheng, X., Cheng, S., 2012. Use of threedimensional excitation and emission matrix fluorescence spectroscopy for predicting 
the disinfection by-product formation potential of reclaimed water. Water Research 46, 5765.

Harman, B.I., Koseoglu, H., Yigit, N.O., Sayilgan, E., Beyhan, M., Kitis, M., 2010. The removal of disinfection by-product precursors from water with ceramic membranes. Water Science and Technology 62 (3), 547-555.

Hashino, M., Hirami, K., Ishigami, T., 2011. Effect of kinds of membrane materials on membrane fouling with BSA. Journal of Membrane Science 384: 157- 165.

Hashino, M., Hirami, k., M., Katagiri, T., Kubota, N., 2011. Effects of three natural organic matter types on cellulose acetate butyrate microfiltration membrane fouling. Journal of Membrane Science 379, 233- 238.

Hashino, M., Katagiri, T., Kubota, N., 2011. Effect of membrane surface morphology on membrane fouling with sodium alginate. Journal of Membrane Science 366, 258265.

Henderson, R., Baker, A., Parsons, S., Jefferson, B., 2008. Characterisation of algogenic organic matter extracted from cyanobacteria, green algae and diatoms. Water Research 42, 3435-3445. 
Hermia J. 1982. Constant pressure blocking filtration laws application to power-law non-newtonian fluids. Transactions of the Institution of Chemical Engineers 60, 183187.

Hilal, N., Oluwaseun, O., J. Miles, N., Nigmatullin, R., 2005. Methods employed for control of fouling in MF and UF membranes: A Comprehensive review. Separation Science and Technology 40 1957-2005.

Hofs, B., Ogier, J., Vries, D., 2011. Comparison of ceramic and polymeric membrane permeability and fouling using surface water. Separation and Purification Technology 79: 365-374.

Huang, H., Lee, N., Young, T., Gary, A., Lozier, J.C., Jacangelo, J.G., 2007. Natural organic matter fouling of low-pressure, hollow-fibre membranes: effects of NOM source and hydrodynamic conditions. Water Research 41 (17) 3823-3832.

Huang, H., Young, T.A., Jacangelo, J.G., 2008 Unified membrane fouling index for low pressure membrane filtration of natural waters: Principles and methodology. Environmental Science Technology 42 714-720.

Jeong, S., Vigneswaran, S., 2015. Practical use of standard pore blocking index as an indicator of biofouling potential in seawater desalination. Desalination 365, 8-14. 
Jin, X., Jawor, A., Kim, S., Hoek, E., 2009. Effects of feed water temperature on separation performance and organic fouling of brackish water RO membranes. Desalination 239, 346-359.

Ju, Y., Hong, I., Hong, S., 2015. Multiple MFI measurements for the evaluation of organic fouling in SWRO desalination. Desalination 365, 136-143.

Karnik, B., Davies, S., Chen, K.C., Jaglowski, D.R., Baumann, M.J., Masten, S.J., 2005. Effects of ozonation on the permeate flux of nano crystalline ceramic membranes. Water Research 39, 728-734.

Katsoufidou, K. S., Sioutopoulos, D. C., Yiantsios, S. G., Karabelas, A. J., 2010. UF membrane fouling by mixtures of humic acids and sodium alginate: Fouling mechanisms and reversibility. Desalination 264(3), 220-227.

Katsoufidou, K., Yiantsios, S. G., Karabelas, A. J., 2007. Experimental study of ultrafiltration membrane fouling by sodium alginate and flux recovery by backwashing. Journal of Membrane Science 300 (1-2), 137-146.

Kenari, S., Barbeau, B., 2016. Understanding ultrafiltration fouling of ceramic and polymeric membranes caused by oxidized iron and manganese in water treatment. Journal of Membrane Science 516, 1-12. 
Kim, J., Davies, S., Baumann, M., Tarabara, V., 2008. Effect of ozone dosage and hydrodynamic conditions on the permeate flux in a hybrid ozonation-ceramic ultrafiltration system treating natural waters. Journal of Membrane Science 311, 165172.

Kimura, K., Hane, Y., Watanabe, Y., Amy, G., Ohkuma, N., 2004. Irreversible membrane fouling during ultrafiltration of surface. Water Research 38, 3431-3441.

Konieczny, K., Bodzek, M., Rejca, M., 2006. A coagulation-MF system for water treatment using ceramic membranes. Desalination 198, 92-101.

Koo, C., Mohammad, A.W., Suja, F., Talib, M., 2012. Review of the effect of selected physicochemical factors on membrane fouling propensity based on fouling indices. Desalination 287, 167-177.

Koo, C., Mohammad, A.W., Suja, F., Talib, M., 2013. Use and development of fouling index in predicting membrane fouling. Separation and Purification Reviews, 42, 296339.

Lee, S., Dilaver, M, Park, P., 2013. Comparative analysis of fouling characteristics of ceramic and polymeric microfiltration membranes using filtration models. Journal of Membrane Science 432, 97-105. 
Lee, S., Kim, J., 2014. Differential natural organic matter fouling of ceramic versus polymeric ultrafiltration membranes. Water Research 48, 43-51.

Lehman, S., Adham, S., Liu, L., Fujuira, S., and Kanaya, S., 2007. Performance of new generation ceramic membranes using coagulation and ozonation pretreatment. American Water Works Association (AWWA) Membrane Technology Conference Tampa, Florida, USA.

Lehman, S.G., Liu, L., 2009 Application of ceramic membranes with preozonation for treatment of secondary wastewater effluent. Water Research 43, 2020-2028.

Li, W., Ling, G., Lei, F., Li, N., Peng, W., Li, K., Lu, H., Hang, F., Zhang, Y., 2018. Ceramic membrane fouling and cleaning during ultrafiltration of limed sugarcane juice. Separation and Purification Technology 190, 9-24.

Liang, H., Gong, W., Chen, J., Li, G., 2008. Cleaning of fouled ultrafiltration (UF) membrane by algae during reservoir water treatment. Desalination 220, 267-272.

Liu, L., Celik, E., Choi, H., 2011. Protein fouling behavior of carbon nanotube/polyethersulfone composite membranes during water filtration. Water Research 45, 5287-5294. 
Loi-Brügger, A., Panglisch, S., Hattori, K., Yonekawa, H., Tomita, Y., and Gimbel, R., 2007. Open Up New Doors in Water Treatment with Ceramic Membranes. Membrane Technology Conference. American Water Works Association, Tampa, Florida, USA.

Lowe, J., Hossain, M., 2008. Application of ultrafiltration membranes for removal of humic acid from drinking water. Desalination 218 343-354.

Ma, Z., Wen, X., Zhao, F., Xia, Y., Huang, X., Wait, D., Guan, J., 2013. Effect of temperature variation on membrane fouling and microbial community structure in membrane bioreactor. Bioresource Technology 133, 462-468.

Markechova, D., Tomkova, M., Sadecka, J., 2013. Fluorecense excitation - emission matrix spectroscopy and parallel factor analysis in drinking water treatment: A review. Journal of Environmental Studies 22, 1289-1295.

Matilainen, A., Gjessing, E., Lathinen, T., Hed, L., Bhatnagar, A., Sillanpaa, M., 2011. An overview of the methods used in the characterisation of natural organic matter (NOM) in relation to drinking water treatment. Chemosphere 83, 1431-1442.

McAliley, I., D’Adamo, P., 2009. Advances in membrane treatment lead to more costeffective options for drinking water providers. AWWA-WEA Annual Conference, Raleigh, NC. 
Meyn, T., Altmann, J., Leiknes, T., 2012. In-line coagulation prior to ceramic microfi ltration for surface water treatment-minimisation of flocculation pre-treatment. Desalination and Water Treatment 42: 163-176.

Meyn, T., Bahn, A., Leiknes, T. O., 2008. Significance of flocculation for NOM removal by coagulation-ceramic membrane microfiltration. Water Science and Technology. Water Supply 8(6), 691-700.

Mo, H., Tay, K., Ng, H., 2008. Fouling of reverse osmosis membrane by protein (BSA): Effects of $\mathrm{pH}$, calcium, magnesium, ionic strength and temperature. Journal of Membrane Science 315, 28-35.

Mosset, A., Bonnelye, V., Petry, M., and Sanz, M.A. 2008. The sensitivity of SDI analysis: from RO feed water to raw water. Desalination 222, 17-23.

Nguyen, A.H., Tobiason, J.E., Howe, K.J., 2011. Fouling indices for low pressure hollow fiber membrane performance assessment. Water Research 45, 2627-2637.

Nigam, M.O., Bansal, B., Chen, X.D., 2008. Fouling and cleaning of whey protein concentrate fouled ultrafiltration membranes. Desalination 218, 313-322.

Peldszus, S., Halle, C., Peiris, R., Hamouda, M., Jin, X., Legge, R, Budman, H., Moresoli, C, Huck, P., 2011. Reversible and irreversible low-pressure membrane 
foulants in drinking water treatment: Identification by principal component analysis of fluorescence EEM and mitigation by biofiltration pretreatment. Water Research 45 , $5161-5170$.

Pendergast, M., Hoek, E., 2011. A review of water treatment membrane nanotechnologies. Energy Environmental Science 4, 1946-1971.

Pifer A.D., Fairey, J.L., 2012. Improving on SUVA254 using fluorescence-PARAFAC analysis and asymmetric flow field flow fractionation for assessing disinfection by product formation and control. Water Research 46, 2927.

Porcelli, N., Judd, S., 2010. Chemical cleaning of potable water membranes: the cost benefit of optimisation, Water Research 44, 1389-1398.

Puspitasari V., Granville A., Le-Clech P., Chena V., 2010. Cleaning and ageing effect of sodium hypochlorite on polyvinylidene fluoride (PVDF) membrane. Separation and Purification Technology 72, 301-308.

Rachman, R., 2011. Assessment of silt density index (SDI) as fouling propensity parameter in reverse osmosis desalination (MASc thesis). King Abdullah University of Science and Technology, Saudi Arabia.

Regula, C., Carretier, E., Wyart, Y., Gesan-Guiziou, G., Vincent, A., Boudot, D., 
Moulin, P., 2014. Chemical cleaning / disinfection and ageing of organic UF membranes: A review. Water Research 56, 325-365.

Rodriguez, S., Amy, G., Schippers, J.C., Kennedy, M., 2015. The Modified Fouling Index Ultrafiltration constant flux for assessing particulate/colloidal fouling of RO systems. Desalination 365, 79-91.

Rodriguez-Salinas, S., Al-Rabaani, B., Kennedy, M., Amy, G., Schippers, J., 2015. MFI-UF constant pressure at high ionic strength conditions. Desalination and Water Treatment 10, 64-72.

Rubia, A., Rodriguez, M., Prats, D., 2006. pH, Ionic strength and flow velocity effects on the NOM filtration with $\mathrm{TiO} 2 / \mathrm{ZrO} 2$ membranes. Separation and Purification Technology 52, 325-331.

Sanchez-Polo, M., Rivera-Utrilla, J., von Gunten, U., 2006. Metal-doped carbon aerogels as catalysts during ozonation processes in aquatic solution. Water Research 40, 3375- 3384 .

Sartor, M., Gatjal, H., Mavrov, V., 2008. Demonstration of a new hybrid process for the decentralised drinking and service water production from surface water in Thailand. Desalination 222, 528-540. 
Schippers, J.C. and Verdouw, J. 1980. The modified fouling index, a method of determining the fouling characteristics of water. Desalination 32: 137-148.

Sentana, I., Puche, R., Sentana, E., Parts, D., 2011. Reduction of chlorination byproducts in surface water using ceramic nanofiltration membranes. Desalination 277 $147-155$.

Shao, J., Hou, J., Song, H., 2011. Comparison of humic acid rejection and flux decline during filtration with negatively charged and uncharged ultrafiltration membranes. Water Research 45, 473 -482.

Shi, X., Tal, G., Hankins, N., Gitis, V., 2014. Fouling and cleaning of ultrafiltration membranes: A review. Journal of Water Process Engineering 1, 121-138.

Sim, 1., Ye, Y., Chen, V., Fane, A., 2010. Crossflow sampler modified fouling index ultrafiltration (CFS-MFIUf) - an alternative fouling index. Journal of Membrane Science 360, 174-184.

Sim, L.N., Ye, Y., Chen, V., and Fane, A.G., 2011 Comparison of MFI-UF constant pressure, MFI-UF constant flux and Crossflow Sampler-Modified Fouling. Water Research 45, 1639-1650. 
Sioutopoulos, D., Karabelas, A., Yiantsios, S., 2010. Organic fouling of RO membranes: Investigating the correlation of RO and UF fouling resistances for predictive purposes. Desalination 261, 272-283.

Steinhauer, T., Hanely, S., Bogendorfer, K., Kulozik, U., 2015. Temperature dependent membrane fouling during filtration of whey and whey proteins. Journal of Membrane Science 492, 364-370.

Subhi, N., Leslie, G., Chen, V., Le-Clech, P., 2013. Organic fouling of ultrafiltration membrane: detailed characterization by liquid chromatography with organic carbon detection (LC-OCD). Separation Science and Technology 48, 199-207.

Taheri, A., Sim, L.N., Haur, C., Alkhondi, E., Fane, A., 2013. The fouling potential of colloidal silica and humic acid and their mixtures. Journal of Membrane Science 433, $112-120$.

Tian, J., Ernst, M., Cui, F., \& Jekel, M., 2013. Effect of different cations on UF membrane fouling by NOM fractions. Chemical Engineering Journal 223, 547-555.

Trang, V., Phuong, L., Dan, N., Thanh, B., Visvanathan, C., 2012. Assessment on the trihalomethanes formation potential of Tan Hiep Water Treatment Plant. Journal of Water Sustainability 2, 43-53. 
Uyguner, C., Suphandag, S., Kerc, A., Bekbolet, M., 2007. Evaluation of adsorption and coagulation charachteristcs of humic acids proceded by alternative oxidation techniques. Desalination 210, 183-193.

Van de Ven, W, Sant K., Punt I., Zwijnenburg A., Kemperman A., Van der Meer, W., 2008. Hollow fiber dead-end ultrafiltration: Influence of ionic environment on filtration of alginates. Journal of Membrane Science 308, 218-229.

Van den Brink, P., Satpradit, O., Bentem, A., Zwijinenburg, A., Temmink, H., Loosdrecht, M., 2011. Effect of temperature shocks on membrane fouling in membrane bioreactors. Water Research 45, 4491-4500.

Van der Bruggen, B., Vandecasteele, C., Gestel, T., Doyen, W., Leysen, R., 2003. A Review of pressure-driven membrane processes in wastewater treatment and drinking water production. Environmental Progress (V01.22, No.1).

Van Geluwe, S., Braeken, L., Van der Bruggen, B., 2011. Ozone oxidation for the alleviation of membrane fouling by natural organic matter: A review. Water Research, $45,3551-3570$.

Vasanth, D., Pugazhenthi, G., Uppaluri, R., 2013. Cross-flow microfiltration of oil-inwater emulsions using low cost ceramic membranes. Desalination 320, 86-95. 
Villacorte, L., Ekowati, Y., Winters, H., Amy, G., Schippers, J., Kennedy, M., 2015. $\mathrm{MF} / \mathrm{UF}$ rejection and fouling potential of algal organic matter from bloom-forming marine and freshwater algae. Desalination 367, 1-10.

Wang, Q., Zeng H., Wua, Z., Cao, J., 2018. Impact of sodium hypochlorite cleaning on the surface properties and performance of PVDF membranes. Applied Surface Science 428, 289-295.

Wang, X., Li, X., Huang, X., 2007. Membrane fouling in a submerged membrane bioreactor (SMBR): Characterisation of the sludge cake and its high filtration resistance. Separation and Purification Technology 52, 439-445.

Wang, X., Mab, J., Wang, Z., Chena, H., Liuc, M., Wu, Z., 2018. Re-investigation of membrane cleaning mechanisms using $\mathrm{NaOCl}$ : Role of reagent diffusion. Journal of Membrane Science 550, 278-285.

Wei, X., Wang, R., Li, Z., Fane, A.G., 2006. Development of a novel electrophoresisUV grafting technique to modify PES UF membranes used for NOM removal. Journal of Membrane Science 273 (1-2), 47-57.

Xia, S., Zhou, Y., Ma, R., Xie, Y., 2013. Ultrafiltration of humic acid and surface water with tubular ceramic membrane. Desalination and Water Treatment 51, 5319-5326. 
Xiao, K., Wang, X., Huang, X., Waite, T., Wen, X., 2009. Analysis of polysaccharide, protein and humic acid retention by microfiltration membranes using Thomas' dynamic adsorption model. Journal of Membrane Science 342, 22-34.

Xiao, P., Xiao, F., Wang, D., Qin, T., He, S., 2012. Investigation of organic foulants behavior on hollow-fiber UF membranes in a drinking water treatment plant. Separation and Purification Technology 95, 109-117.

Yiantsios, S.G., Sioutopoulos, D., and Karabelas, A.J. 2005. Colloidal fouling of RO membranes: an overview of key issues and efforts to develop improved prediction techniques. Desalination 183, 257-272.

Yin, N., Zhong, Z., Xing, W., 2013. Ceramic membrane fouling and cleaning in ultrafiltration of desulfurization wastewater. Desalination 319, 92-98.

Yuan, W., Zydney, A., 2000. Humic acid fouling during ultrafiltration. Environmental Science and Technology 34, 5043-5050.

Zepp, R., Sheldon, W., Moran, M., 2004. Dissolved organic fluorophores in southeastern US coastal waters: correction method for eliminating Rayleigh and Raman scattering peaks in excitation-emission matrices. Marine Chemistry 89, 15-36.

Zhang, T., Lu, J., Ma, J., Qiang, Z., 2008. Fluorescence spectroscopic characterization 
of DOM fractions isolated from a filtered river water after ozonation and catalytic ozonation. Chemosphere 71, 911-921.

Zhang, X., Guo, J., Wang, L., Hu, J., Zhu, J., 2013. In situ ozonation to control ceramic membrane fouling in drinking water treatment. Desalination 328, 1-7.

Zhao, S., Zou, L., 2011. Effects of working temperature on separation performance, membrane scaling and cleaning in forward osmosis desalination. Desalination 278, 157164.

Zhou, J., Chang, Q., Wang, Y., Wang, J., Meng, G., 2010. Separation of stable oil-water emulsion by the hydrophilic nano-sized $\mathrm{ZrO}_{2}$ modified $\mathrm{Al}_{2} \mathrm{O}_{3}$ microfiltration membrane. Separation and Purification Technology 75 (3), 243-248.

Zularisam, A., Ahmad, A., Sakinah, M., Ismail, A. F., Matsuura, T., 2011. Role of natural organic matter (NOM), colloidal particles, and solution chemistry on ultrafiltration performance. Separation and Purification Technology 78(2), 189-20.

Zularisam, A., Ismail, A.F., Salim, R., 2006. Behaviours of natural organic matter in membrane filtration for surface water treatment — a review. Desalination 194-211-231. 


\section{Appendix (A) \\ Experimental Methods}

\section{A.1 Preparation of feed water solutions}

Humic acids (2.5 mg C/L), a protein (bovine serum albumin, BSA, $2.5 \mathrm{mg} \mathrm{C} / \mathrm{L}$ ), a polysaccharide (sodium alginate, $2.5 \mathrm{mg} \mathrm{C} / \mathrm{L}$ ), and a mixture of the three NOM models ( $0.83 \mathrm{mg} \mathrm{C/L} /$ each NOM model, total of $2.5 \mathrm{mg} \mathrm{C} / \mathrm{L})$, were used as model NOM foulants. A moderate hardness and alkalinity of $75 \mathrm{mg} / \mathrm{L}$ calcium carbonate $\left(\mathrm{CaCO}_{3}\right)$ and a low level of turbidity ( $5 \mathrm{NTU})$ as kaolin clay particles were included in the synthetic water matric. All model substances were purchased from Sigma Aldrich. Feed solutions were prepared using DI water and were mixed using a magnetic stirrer one-day prior any experiment to ensure that materials were dissolved completely. During filtration experiments, feed water was continuously mixed using a VWR dual speed mixer to ensure homogeneous water conditions throughout the experiment. The $\mathrm{pH}$ of the feed was adjusted as needed to 7.5 with $\mathrm{NaOH}$ and monitored using the HACH (cat.no. 58258-00) HQd Field Case equipment.

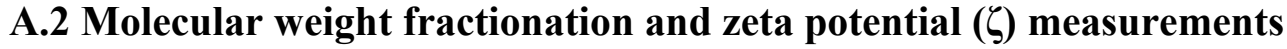

$2 \mathrm{~L}$ of feed water solutions was prepared for the fractionation testing. The molecular weight distribution of different feed solutions was determined by ultrafiltration (UF) fractionation method using a $400 \mathrm{~mL}$ UF stirred cell (model 8400, Amicon Inc.) and five hydrophilic regenerated cellulose membranes (Millipore, Bradford MA) with different molecular weight cut offs (MWCOs) of 1,000, 5,000, 10,000, 30,000, and 100,000 Daltons (Figure A.1). The fractionation was performed sequentially by passing $400 \mathrm{~mL}$ of sample through the membrane with the highest MWCO to that with the lowest MWCO. Samples from 
permeate and retentate of particular membrane were collected and total organic carbon (TOC) was analyzed using Tekmar Dohrmann, Phoenix 8000 TOC analyzer. Excess permeate produced by each fractionation step was used as the feed to the next membrane with a lesser MWCO. The TOC concentration of a particular molecular weight fraction was calculated by subtracting the TOC concentration of the filtrate from one membrane from the TOC concentration of the filtrate from the membrane of the next larger nominal MWCO. After fractionation experiments, membranes were rinsed with Milli-Q water for 60 minutes changing the water three times (i.e. every 20 minutes) and stored in $10 \%$ by volume ethanol/water solution and kept in refrigerator at $4{ }^{\circ} \mathrm{C}$ for later use. Prior to use, the membranes were rinsed several times after which Milli-Q water was allowed to filtered through them for three times.
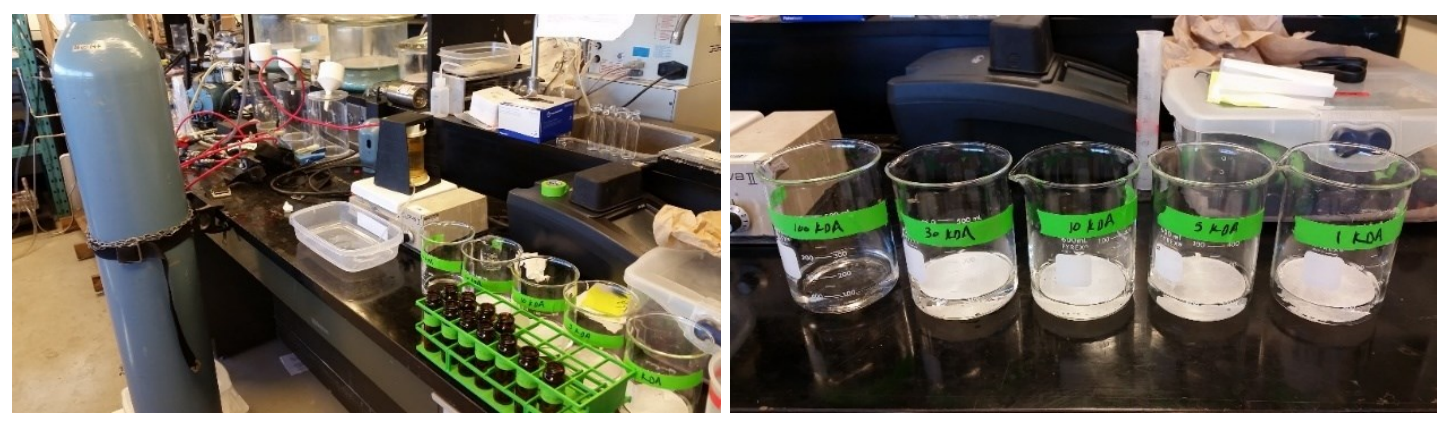

Figure A.1- Molecular weight fractionation setup and UF membranes

A Malvern Zetasizer Nano was used to determine the zeta potential $(\zeta)$ of feed water solutions. In summary, an electric field is applied via electrodes immersed in a sample and this causes the charged particles to move towards the electrode of opposite polarity. Particle mobility is determined from the Smoluchowski model calculated by the Zetasizer equipment. Triplicate measurements were performed for each sample. 


\section{A.3 MFI-UF testing}

The MFI-UF setup (Figure A.2) consisted of the following components: a digital gear pump (Cole Parmer: Drive No. 75211-30, Head No. 07003-04); a ball valve (Cole Parmer No. 01377-18); a pressure relief valve (Aquatrol No. 3ETU4), and a pressure regulator with gauge (Veolia No. LA512). PAN UF, hollow fiber, inside-out membrane module (Microza, Pall Corp.) with a MWCO of $13 \mathrm{kDa}$ was used. High-capacity precision top loading balance (Adam Equipment NBL8201e) to measure the permeate volume. The MFI-UF testing was performed under constant pressure (variable temperature) or constant temperature (variable pressure). The pressure was regulated and monitored using the pressure regulator and the gauge while water temperature was controlled using either an immersion heater (Cole Parmer) or a compact chiller (LM series, Polyscience) as required. Feed water was filtered through the UF membrane under dead-end mode while monitoring flux decline during the test. Permeate was collected in a tank set on the electronic balance which has an RS 232 interface with a computer in order to acquire permeate volume (V) and filtration time (t) data from the balance. Data were recorded every 1 minute and imported into MS Excel spread sheet with data terminal software (TeraTerm). The MFI-UF was then calculated from the inverse of the flow rate $(\mathrm{t} / \mathrm{V})$ versus the permeate volume $(\mathrm{V})$ recorded by the balance. 

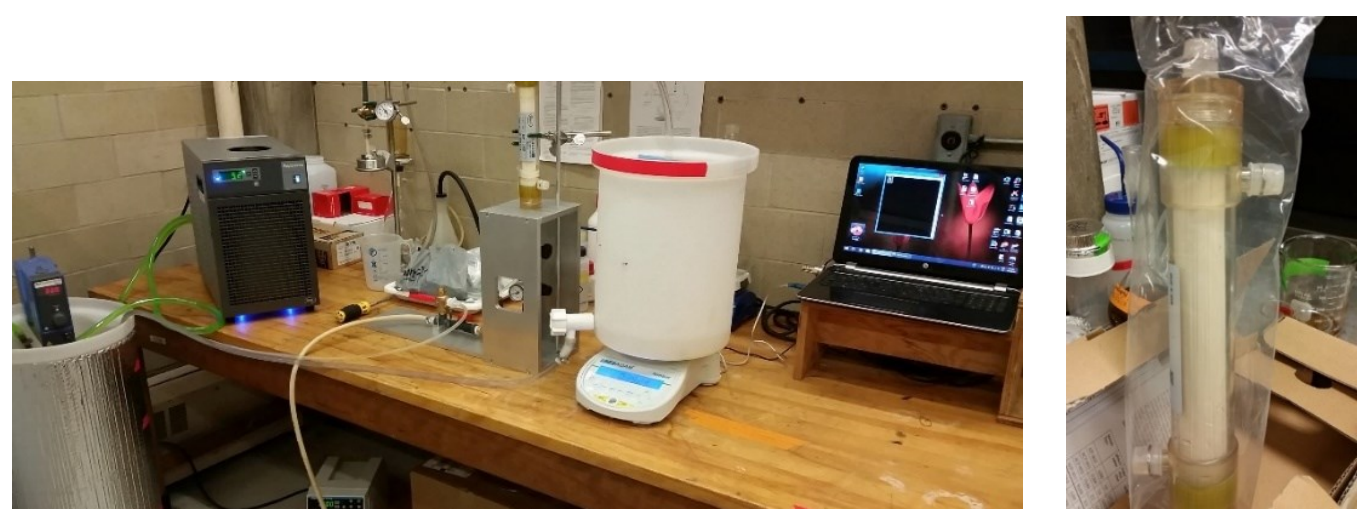

Figure A.2 - MFI-UF setup and 13 kDa UF membrane

\section{A.4 Submerged Polymeric UF Membranes Testing}

Two submerged membrane systems were used for the testing (Figure A.3). Zenon ZW-1 hollow fiber (UF) membranes made of polyvinylidene fluoride (PVDF), with a nominal pore size of $0.04 \mu \mathrm{m}$ (surface area of $0.047 \mathrm{~m}^{2}$ ) were used and operated under a vacuum pressure between 0 and -8.7 psi ( 0 and 0.6 bar). Filtration experiments were performed for 24 hrs under the following operational conditions presented: constant flux (38 LMH); backwash (38 LMH every 15 minutes for 20 seconds); air scour (5 L/minute). The flow rate and direction of permeate (i.e. forward and backward) were controlled using WinLN software. Pressure readings were recorded every 10 seconds using Operational Flux 2.0 program. A macro filter code (shown in Appendix C) was used to analyze filtration data (i.e. removing noisy data).

Membranes were cleaned in place (CIP) between each experiment following manufacturer specified recommendations. Cleaning of the membrane units was done in two steps: soaking in a $250 \mathrm{ppm}$ of sodium hypochlorite $(\mathrm{NaOCl})$ solution for 4 hours followed by soaking in $1 \%$ citric acid solution for another 4 hours. Chemical cleaning was conducted 
at room temperature. DI water was filtered through the membrane after each cleaning until a permeate flux of $38 \mathrm{LMH}$ was obtained; the permeate flux was determined manually by measuring the volume produced over 1 minute. Repeat cleanings were conducted until the flux $38 \pm 0.50 \mathrm{LMH}$ was reached.
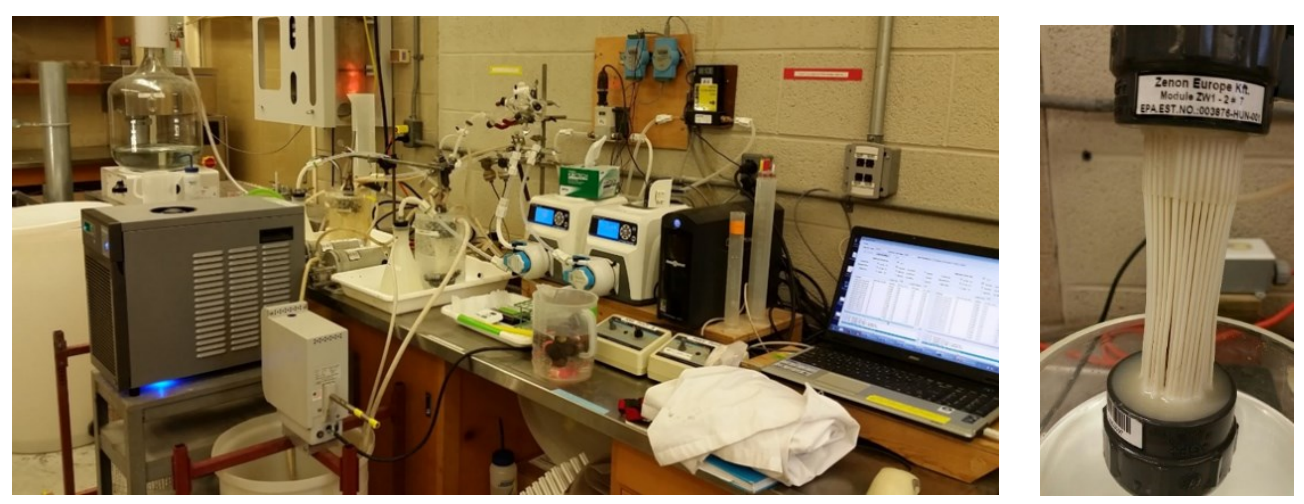

Figure A.3 - Automated submerged polymeric UF system and Zenon ZW-1 membrane

\section{A.5 Ceramic and Polymeric UF Membranes Testing}

Experiments were conducted on an automated filtration system (Figure A.4) consisting of a digital gear pump (Cole Parmer: Drive no. 75211-30, Head no. 07003-04), pressure transducer (Omega: model no. PX409-100G5V), flow meter (Cole Parmer: model no. 32703-52), solenoid valves (Macmaster: model no. 4711K731), and pressure vessel (Cole Parmer: model no. 29902-90) and nitrogen gas for backwash. Labview program code (National Instruments, NI, USA) was created to operate the system automatically such as controlling the feed pump to have a constant flux, and automatically switches from filtration cycles to backwash cycles while recording flux and pressure data. A ceramic UF $\left(\right.$ Ceramem $^{\mathrm{TM}}$, Veolia) and polymeric UF (X-Flow ${ }^{\mathrm{TM}}$, Pentair) were used. Fouling experiments were performed for 24 hours under the following conditions: constant flux of 
$100 \mathrm{LMH}$; dead-end filtration mode; and backwash using pressurized water at 30 psi for 20s every $4 \mathrm{hrs}$. of filtration cycle $\left(400 \mathrm{~L} / \mathrm{m}^{2}\right)$. Clean water flux (CWF) was measured before and between experiments to determine the transmembrane pressure (TMP) versus flux relationship for a clean membrane and to estimate the intrinsic membrane resistance.

CIP cleaning was performed using two common organic fouling cleaners: an oxidant, $\mathrm{NaOCl}$ or caustic cleaning, $\mathrm{NaOH}$. Each cleaning was performed for 4 hours at a cleaning temperature of $35^{\circ} \mathrm{C}$ and $\mathrm{pH}$ of 11.0 for both cleaning solutions. Chemicals were recirculated at crossflow velocity of $0.1 \mathrm{~m} / \mathrm{s}$ for 1 hour followed by 3 hours of soaking. For the $\mathrm{NaOCl}$ chemical wash, the free chlorine concentration was $500 \mathrm{mg} \mathrm{Cl} / \mathrm{L}$ which was measured before testing using the $\mathrm{HACH}$ method (8021) and a digital colorimeter ${ }^{\mathrm{TM}} \mathrm{II}$.

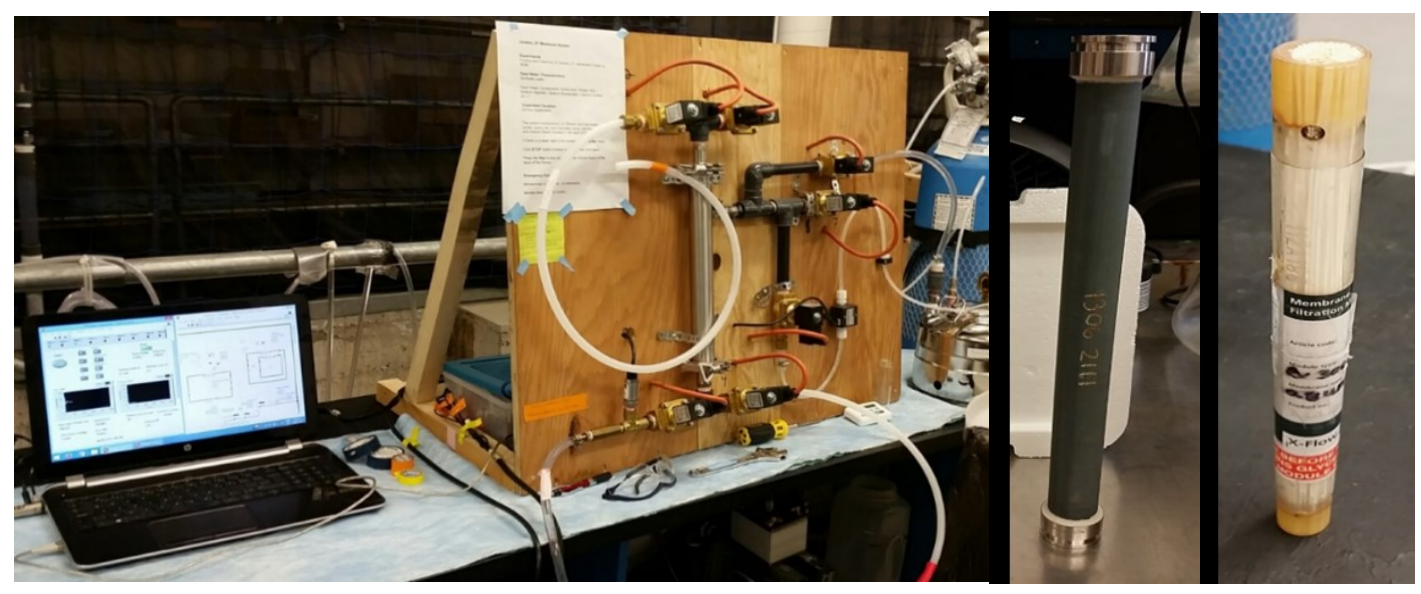

Figure A.4 - Automated ceramic UF system. Ceramem (Veolia) and X-Flow (Pentair) membranes. 


\section{A.6 Ceramic UF CIP Testing ( $\mathrm{NaOCl}, \mathrm{NaOH}$, and $\left.\mathrm{O}_{3}\right)$}

The cleaning efficiency of $\mathrm{NaOCl}$ and $\mathrm{NaOH}$ in a single, stepwise, and sequential cleaning was examined. $\mathrm{NaOCl}$ was purchased as a solution $(6 \%)$. The concentration of free chlorine in the wash water $\left(500 \mathrm{mg} \mathrm{Cl}_{2} / \mathrm{L}\right.$, as free chlorine) was measured before testing using the $\mathrm{HACH}$ method (8021) and a digital colorimeter ${ }^{\mathrm{TM}}$ II. Single cleaning using $\mathrm{NaOCl}$ or $\mathrm{NaOH}$, (pH 11 or 12), was performed alternatively for 4 hours. $\mathrm{NaOCl} / \mathrm{NaOH}$ sequential cleaning was performed with $500 \mathrm{mg} \mathrm{Cl} / \mathrm{L} \mathrm{NaOCl}(\mathrm{pH}$ 12) for 2 hours followed by $\mathrm{NaOH}$ ( $\mathrm{pH}$ 12) for 2 hours (total of 4 hours). $\mathrm{NaOH} / \mathrm{NaOCl}$ sequential cleaning was performed with $\mathrm{NaOH}(\mathrm{pH} \mathrm{12)}$ for 2 hours followed by $500 \mathrm{mg} \mathrm{Cl} 2 / \mathrm{L} \mathrm{NaOCl}(\mathrm{pH} \mathrm{12})$ for 2 hours (total of 4 hours). For combined cleaning, $(\mathrm{NaOCl}+\mathrm{NaOH})$, $\mathrm{NaOCl}$ was simultaneously mixed with $\mathrm{NaOH}(\mathrm{pH} 12)$, i.e. both chemicals were added concurrently at the same concentration as in their single solution, and the cleaning duration was maintained at a 4 hours duration to ensure the same chemical contact time for direct comparisons with individual cleaning solutions. Chemicals were prepared in $4 \mathrm{~L}$ of DI water and recirculated in a closed loop at a cross flow velocity of $0.1 \mathrm{~m} / \mathrm{s}$ for 1 hour followed by 3 hours soak. Cleaning was performed at $35^{\circ} \mathrm{C}$ water temperature.

$\mathrm{O}_{3}$ CIP cleaning efficiency was examined and compared to $\mathrm{NaOCl}$ and/or $\mathrm{NaOH}$. For each $\mathrm{O}_{3}$ cleaning experiment, $4 \mathrm{~L}$ of ozonated water was first prepared by bubbling at $4{ }^{\circ} \mathrm{C} \mathrm{a} 6 \%$ ozone/oxygen gas (Ozone Solutions, USA) in DI water to saturation (Figure A.5). Cleaning was performed by recirculating $\mathrm{O}_{3}$ injected at either $0.25 \mathrm{mg} \mathrm{O} / \mathrm{mg} \mathrm{C}$ or $0.50 \mathrm{mg} \mathrm{O} / \mathrm{mg}$ $\mathrm{C}$, where $\mathrm{mg} \mathrm{C}$ is the carbon mass accumulated on the membrane surface (i.e. $\mathrm{O}_{3}$ concentration in the $4 \mathrm{~L}$ cleaning solution ranges from $15-30 \mathrm{mg} \mathrm{O}_{3} / \mathrm{L}$ and $30-60 \mathrm{mg} \mathrm{O} / \mathrm{L}$ 
for the 0.25 and $0.50 \mathrm{mg} \mathrm{O}_{3} / \mathrm{mg} \mathrm{C}$ ratios respectively). Recirculation of $\mathrm{O}_{3}$ was performed in a closed loop for 1 hour at a cleaning water temperature of $15{ }^{\circ} \mathrm{C} . \mathrm{O}_{3}$ residuals were measured using the standard indigo trisulfonate method (Bader and Hoigin, 1981). Total organic carbon (TOC) analyses were performed during $\mathrm{O}_{3}$ cleaning by collecting a sample at different time intervals (i.e. 1, 5, 10 minutes, etc.) to determine the cumulative $\mathrm{O}_{3}$ demand and irreversible carbon removal as a function of cleaning time.
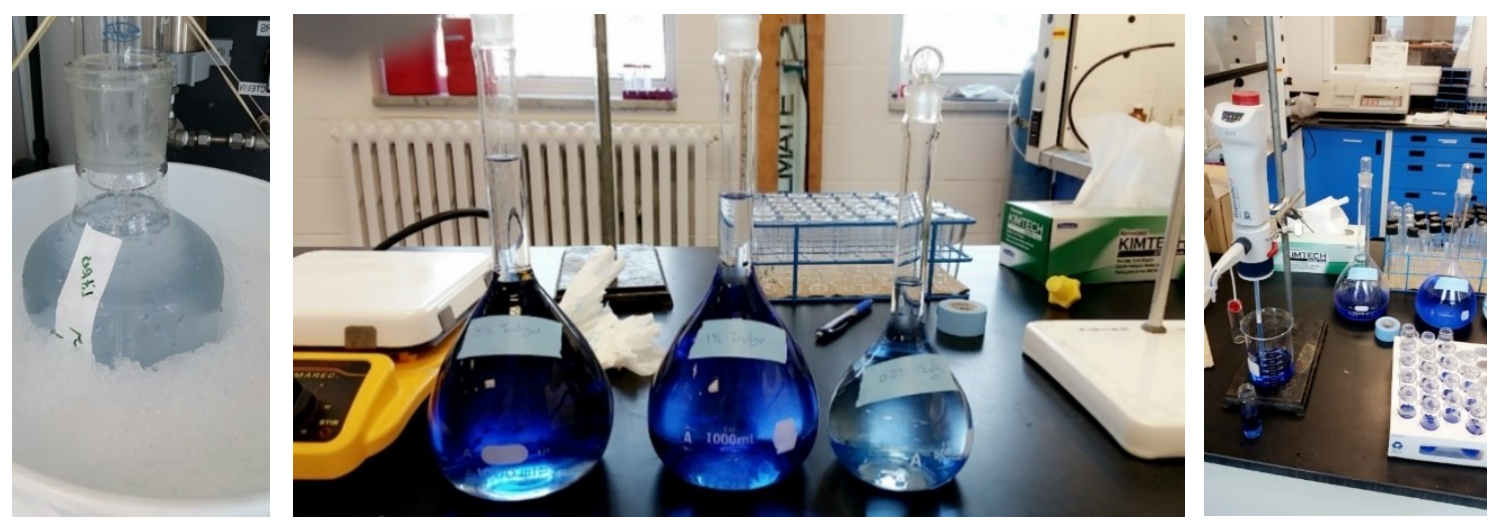

Figure A.5 - Ozone and indigo solutions preparation 


\section{Appendix (B)}

\section{MFI-UF Statistical Analysis Summary}

Table B1 - ANOVA and multiple Comparisons (Tukey HSD) 95\% confidence interval)

\begin{tabular}{ccccc}
\hline $\begin{array}{c}\text { Operating } \\
\text { Pressure (Bar) }\end{array}$ & $\begin{array}{c}\text { MFI-UFHA } \\
\text { p-value } \\
(\mathbf{0 . 0 0 2}) *\end{array}$ & $\begin{array}{c}\text { MFI-UFBSA } \\
\text { p-value } \\
\mathbf{( 0 . 0 0 1 ) *}\end{array}$ & $\begin{array}{c}\text { MFI-UFSA } \\
\text { p-value } \\
(\mathbf{0 . 0 0 8} *\end{array}$ & $\begin{array}{c}\text { MFI-UFMix } \\
\text { p-value } \\
\mathbf{( 0 . 0 0 1 ) *}\end{array}$ \\
\hline 1 vs. 2 & 0.010 & 0.001 & 0.036 & 0.005 \\
1 vs. 3 & 0.001 & 0.002 & 0.007 & 0.001 \\
2 vs. 3 & 0.010 & 0.001 & 0.046 & 0.004 \\
\hline
\end{tabular}

*p-value of all data set for each NOM model

Table B2 - ANOVA and multiple Comparisons (Tukey HSD) (95\% confidence interval)

\begin{tabular}{|c|c|c|c|c|}
\hline $\begin{array}{c}\text { Water } \\
\text { Temperature } \\
\left({ }^{\circ} \mathrm{C}\right)\end{array}$ & $\begin{array}{c}\text { MFI-UFHA } \\
\text { p-value } \\
(0.001) *\end{array}$ & $\begin{array}{c}\text { MFI-UFBSA } \\
\text { p-value } \\
(0.002) *\end{array}$ & $\begin{array}{c}\text { MFI-UFsA } \\
\text { p-value } \\
(0.001)^{*}\end{array}$ & $\begin{array}{c}\text { MFI-UFMix } \\
\text { p-value } \\
(0.002) *\end{array}$ \\
\hline 5 vs. 10 & 0.005 & 0.027 & 0.023 & 0.046 \\
\hline 5 vs. 20 & 0.001 & 0.001 & 0.005 & 0.001 \\
\hline 5 vs. 30 & 0.003 & 0.0003 & 0.001 & 0.002 \\
\hline 5 vs. 35 & 0.002 & 0.002 & 0.001 & 0.001 \\
\hline 10 vs. 20 & 0.042 & 0.012 & 0.031 & 0.009 \\
\hline 10 vs. 30 & 0.007 & 0.003 & 0.004 & 0.002 \\
\hline 10 vs. 35 & 0.006 & 0.001 & 0.004 & 0.001 \\
\hline 20 vs. 30 & 0.030 & 0.036 & 0.041 & 0.037 \\
\hline 20 vs. 35 & 0.044 & 0.041 & 0.035 & 0.040 \\
\hline 30 vs. 35 & 0.042 & 0.038 & 0.050 & 0.045 \\
\hline
\end{tabular}

*p-value of all data set for each NOM model

Table B3 - Regression coefficients for the predicted MFI-UF models

\begin{tabular}{cccc}
\hline & \multicolumn{3}{c}{ Regression Coefficients } \\
\cline { 2 - 4 } Model NOM & $\begin{array}{c}\text { Unstandardized } \\
\boldsymbol{\beta}_{\mathbf{0}}^{*}\end{array}$ & $\begin{array}{c}\text { Unstandardized } \\
\text { (Standardized) } \\
\boldsymbol{\beta}_{\mathbf{1}}^{*}\end{array}$ & $\begin{array}{c}\text { Unstandardized } \\
\text { (Standardized) } \\
\boldsymbol{\beta}_{2}^{*}\end{array}$ \\
\hline Humic Acid & 1588.858 & $2058.965(0.652)$ & $-121.343(-0.623)$ \\
Protein (BSA) & 3351.687 & $3681.250(0.721)$ & $-201.537(-0.691)$ \\
Sodium Alginate & 1189.372 & $3139.250(0.755)$ & $-140.453(-0.609)$ \\
Mixture & 2598.917 & $3537.500(0.739)$ & $-173.452(-0.653)$ \\
\hline
\end{tabular}

$* \beta_{0}$ : intercept; $\beta_{1}$ : pressure effect; $\beta_{2}$ : temperature effect 


\section{MFI-UF GLM Models (SPSS Output)}

ANOVA $^{\mathrm{a}}$

\begin{tabular}{ll|r|r|r|r|r} 
Model & & Sum of Squares & df & Mean Square & F & \multicolumn{1}{c}{ Sig. } \\
\hline 1 & Regression & 18049366.249 & 2 & 9024683.124 & 24.143 & $.003^{\text {b }}$ \\
\cline { 2 - 7 } & Residual & 1868968.591 & 5 & 373793.718 & & \\
\cline { 2 - 7 } & Total & 19918334.839 & 7 & & & \\
\hline
\end{tabular}

a. Dependent Variable: Humic acid MFIUF

b. Predictors: (Constant), Water Temperature (C), Operating Pressure (Bar)

\section{Coefficients $^{\mathbf{a}}$}

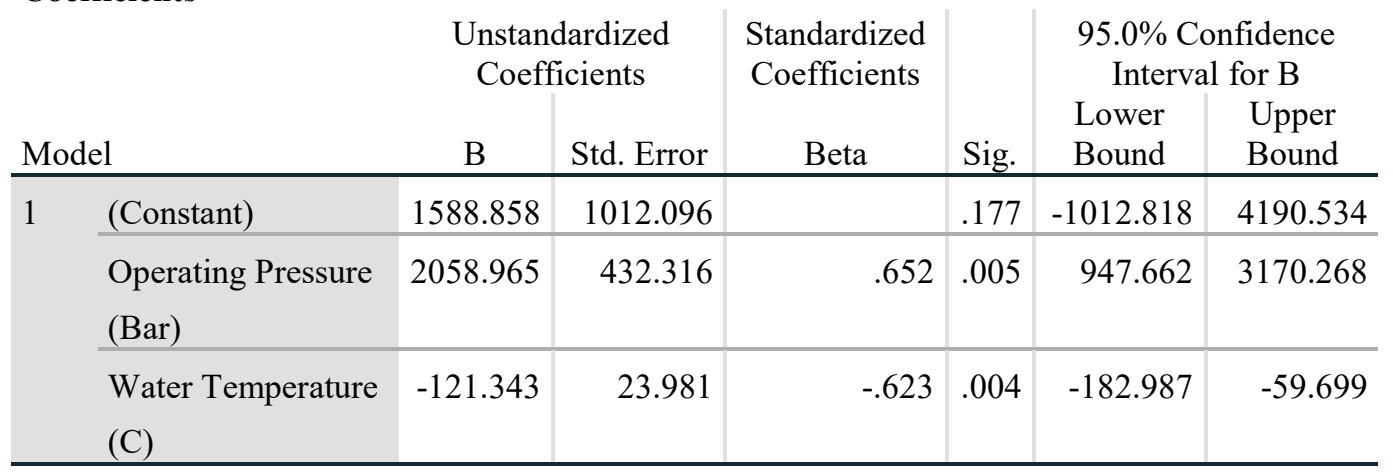

a. Dependent Variable: Humic acid MFIUF

\begin{tabular}{|c|c|c|c|c|c|c|}
\hline \multicolumn{7}{|c|}{ ANOVA $^{a}$} \\
\hline & & Sum of Squares & $\mathrm{df}$ & Mean Square & $\mathrm{F}$ & Sig. \\
\hline \multirow[t]{3}{*}{1} & Regression & 53504398.972 & 2 & 26752199.486 & 77.078 & $.000^{\mathrm{b}}$ \\
\hline & Residual & 1735406.938 & 5 & 347081.388 & & \\
\hline & Total & 55239805.910 & 7 & & & \\
\hline
\end{tabular}

a. Dependent Variable: Protein MFIUF

b. Predictors: (Constant), Water Temperature (C), Operating Pressure (Bar)

\section{Coefficients $^{\mathbf{a}}$}

\begin{tabular}{|c|c|c|c|c|c|c|c|}
\hline & & \multicolumn{2}{|c|}{$\begin{array}{l}\text { Unstandardized } \\
\text { Coefficients }\end{array}$} & \multirow{2}{*}{$\begin{array}{c}\text { Standardized } \\
\text { Coefficients } \\
\text { Beta }\end{array}$} & \multirow[b]{2}{*}{ Sig. } & \multicolumn{2}{|c|}{$\begin{array}{l}95.0 \% \text { Confidence } \\
\text { Interval for B }\end{array}$} \\
\hline & & B & $\begin{array}{l}\text { Std. } \\
\text { Error }\end{array}$ & & & $\begin{array}{l}\text { Lower } \\
\text { Bound }\end{array}$ & $\begin{array}{l}\text { Upper } \\
\text { Bound }\end{array}$ \\
\hline 1 & (Constant) & 3351.687 & 975.262 & & .019 & 844.695 & 5858.678 \\
\hline & $\begin{array}{l}\text { Operating Pressure } \\
\text { (Bar) }\end{array}$ & 3681.250 & 416.582 & .721 & .000 & 2610.391 & 4752.109 \\
\hline & $\begin{array}{l}\text { Water Temperature } \\
\text { (C) }\end{array}$ & -201.537 & 23.108 & -.691 & .000 & -260.938 & -142.137 \\
\hline
\end{tabular}

a. Dependent Variable: Protein MFIUF 
ANOVA ${ }^{a}$

\begin{tabular}{ll|r|r|r|r|r} 
Model & & Sum of Squares & df & Mean Square & F & \multicolumn{1}{c}{ Sig. } \\
\hline \multirow{2}{*}{1} & Regression & 32532374.556 & 2 & 16266187.278 & 39.930 & $.001^{\mathrm{b}}$ \\
\cline { 2 - 7 } & Residual & 2036821.058 & 5 & 407364.212 & & \\
\cline { 2 - 7 } & Total & 34569195.614 & 7 & & & \\
\hline
\end{tabular}

a. Dependent Variable: Sodium Alginate MFIUF

b. Predictors: (Constant), Water Temperature (C), Operating Pressure (Bar)

\section{Coefficients $^{\mathrm{a}}$}

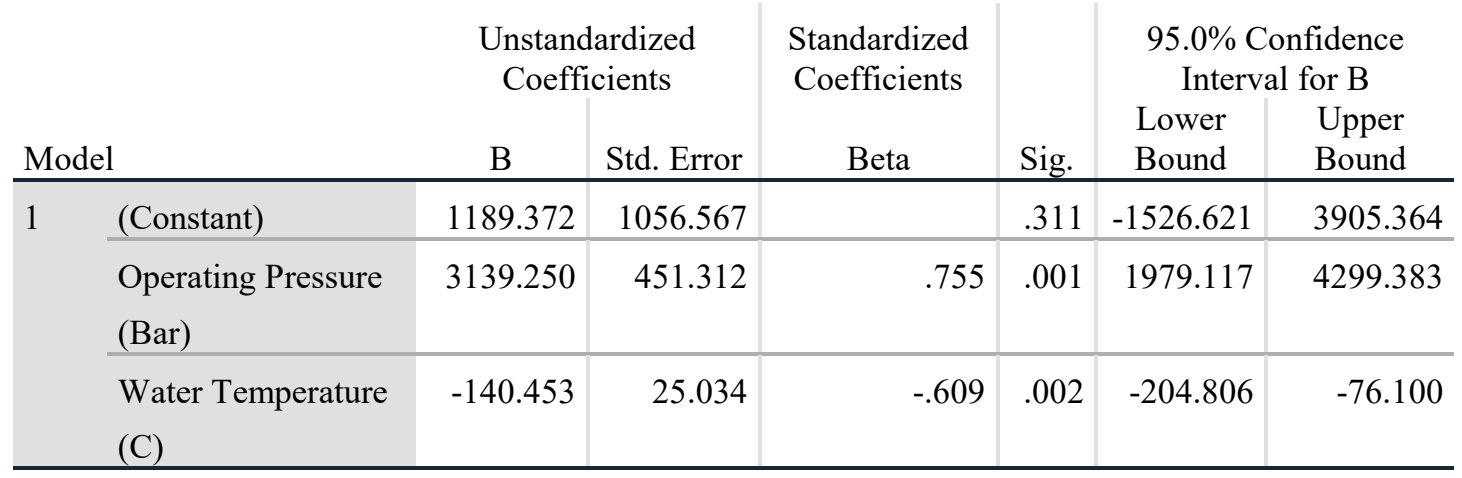

a. Dependent Variable: Sodium Alginate MFIUF

\section{ANOVA}

\begin{tabular}{ll|r|r|r|r|r} 
Model & & Sum of Squares & df & Mean Square & F & \multicolumn{1}{c}{ Sig. } \\
\hline 1 & Regression & 44583554.169 & 2 & 22291777.084 & 89.699 & $.000^{\mathrm{b}}$ \\
\cline { 2 - 7 } & Residual & 1242585.249 & 5 & 248517.050 & & \\
\cline { 2 - 7 } & Total & 45826139.418 & 7 & & & \\
\hline
\end{tabular}

a. Dependent Variable: NOM Mixture MFIUF

b. Predictors: (Constant), Water Temperature (C), Operating Pressure (Bar)

\section{Coefficients $^{\mathbf{a}}$}

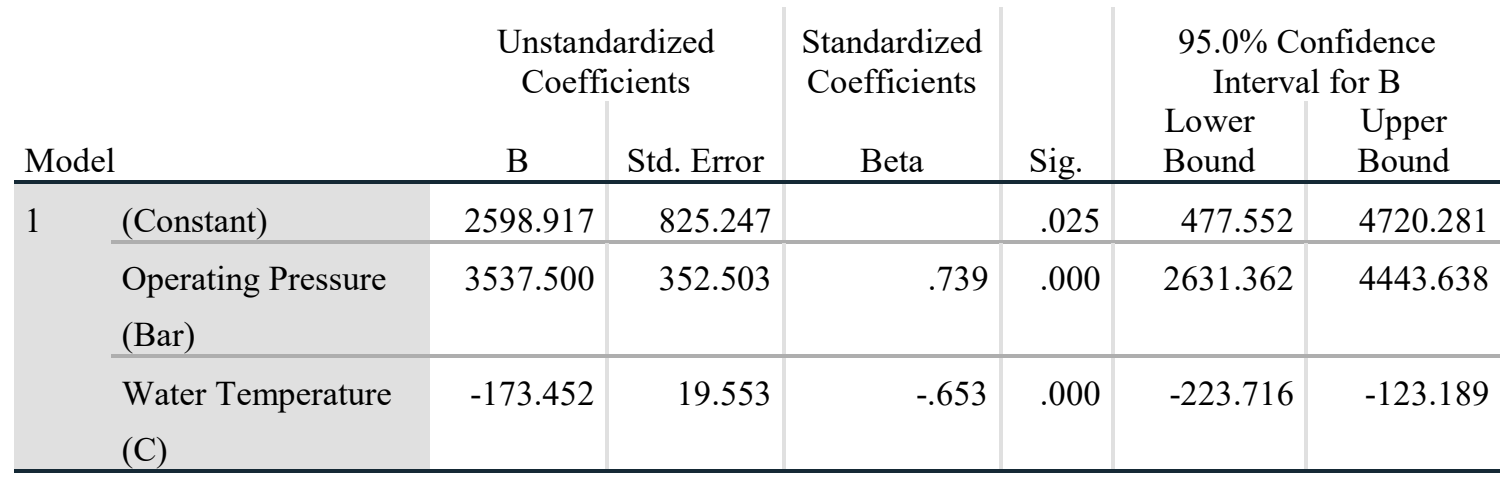

a. Dependent Variable: NOM Mixture MFIUF 


\section{Appendix (C)}

\section{Macro Filter Code used in Fouling Data Analysis}

Sub BWRfilt()

(Created by Kelly Alder and Nigel De Souza)

' BWRfilt Macro

' Authors: Kelly Alder and Nigel De Souza

' Keyboard Shortcut: Ctrl+n

' Create a variable to hold the column id

Dim sColumn As String

Dim sColumnHeader As String

' Create a new variable to hold the cell value

Dim nValue As Double

' Create a new variable to hold percent difference

Dim nPercentDiff As Double

' Create a new variable to hold previos value

Dim nPrevValue As Double

' Create a string representation for the Column Id

sColumn $=$ Chr(ActiveCell.Column +64$)+" 1 "$

' Go to the top of the selected column

Range(sColumn).Select

' Get the text of the column header

sColumnHeader $=$ ActiveCell.Text

' Trim all leading and trailing whitespaces

sColumnHeader $=$ Trim $($ sColumnHeader $)$

' Make the Column HeEader uppercase

' for case-insensitive comparison

sColumnHeader $=$ UCase $($ sColumnHeader $)$

' Make sure we're looking at a Pressure Reasing Column

If sColumnHeader $=$ "PRESSURE_READING" Then

' Move the selected cell one row down

ActiveCell.Offset $(2,0)$.Select

' Check that we haven't reached the end by checking that it's not blank 
While (ActiveCell.Text $<>$ "')

' Get the cell value and previous cell value

nValue $=$ ActiveCell. Value

' Check to see if a row is positive

If $n$ Value $>0$ Then

' Delete the positive row

Rows(ActiveCell.Row).EntireRow.Delete

Else

' Get the value of the previous cell

nPrevValue $=$ ActiveCell.Offset $(-1,0)$.Value

' Check to see if the cell value is greater than

' the previous value

If $n$ Value $>$ nPrevValue Then

'Calculate percent difference

nPercentDiff $=($ nPrevValue - nValue $) /$ nPrevValue $* 100$

' Check to see if percent difference is larger that our tolerance If $n$ PercentDiff $>5$ Then

' Delete this row, we dont want it

Rows(ActiveCell.Row).EntireRow.Delete

Else

' Move the selected cell one row down

ActiveCell.Offset $(1,0)$. Select

End If

Else

' Move the selected cell one row down

ActiveCell.Offset $(1,0)$.Select

End If

End If

Wend

Else

' Display Warning

MsgBox ("Macro Must Be Run On a PRESSURE_READING Column!")

End If

End Sub 


\section{$\underline{\text { Appendix (D) }}$}

\section{Randomized Block Design (RBD) (SPSS Output)}

\section{Between-Subjects Factors}

\begin{tabular}{ccc|c} 
& & Value Label & $\mathrm{N}$ \\
\hline Treatment Membrane Type & 10.00 & Ceramic & 5 \\
\cline { 2 - 4 } & 20.00 & Polymeric & 5 \\
\hline \multirow{2}{*}{ NOM Type } & 1.00 & Humic & 2 \\
\cline { 2 - 4 } & 2.00 & BSA & 2 \\
\hline 3.00 & Alginate+Ca & 2 \\
\hline 4.00 & Alginate-Ca & 2 \\
\hline 5.00 & Mixture & 2 \\
\hline
\end{tabular}

Tests of Between-Subjects Effects

Dependent Variable: \%Removal

\begin{tabular}{cc|c|c|c|c} 
& $\begin{array}{c}\text { Type III Sum of } \\
\text { Squares }\end{array}$ & df & Mean Square & F & Sig. \\
\hline Corrected Model & $495.500^{\mathrm{a}}$ & 5 & 99.100 & 25.740 & .004 \\
\hline Intercept & 50268.100 & 1 & 50268.100 & 13056.649 & .000 \\
\hline Block NOM Type & 399.400 & 4 & 99.850 & 25.935 & .004 \\
\hline Treatment Membrane Type & 96.100 & 1 & 96.100 & 24.961 & .008 \\
\hline Error & 15.400 & 4 & 3.850 & & \\
\hline Total & 50779.000 & 10 & & & \\
\hline Corrected Total & 510.900 & 9 & & & \\
\hline
\end{tabular}

a. R Squared $=.970($ Adjusted R Squared $=.932)$ 


\section{Multiple Comparisons}

\begin{tabular}{|c|c|c|c|c|c|c|}
\hline \multirow[b]{3}{*}{ (I) NOM Type } & \multicolumn{4}{|c|}{$\begin{array}{c}\text { Dependent Variable: \%Removal } \\
\text { Tukey HSD }\end{array}$} & \multirow{2}{*}{\multicolumn{2}{|c|}{$95 \%$ Confidence Interval }} \\
\hline & \multirow[b]{2}{*}{ (J) NOM Type } & \multirow{2}{*}{$\begin{array}{c}\text { Mean } \\
\text { Difference (I-J) }\end{array}$} & \multirow[b]{2}{*}{ Std. Error } & \multirow[b]{2}{*}{ Sig. } & & \\
\hline & & & & & Lower Bound & Upper Bound \\
\hline \multirow[t]{4}{*}{ Humic } & BSA & $-16.0000^{*}$ & 1.96214 & .006 & -24.7229 & -7.2771 \\
\hline & Alginate $+\mathrm{Ca}$ & $-11.5000^{*}$ & 1.96214 & .019 & -20.2229 & -2.7771 \\
\hline & Alginate-Ca & -.5000 & 1.96214 & .039 & -9.2229 & 8.2229 \\
\hline & Mixture & -4.0000 & 1.96214 & .046 & -12.7229 & 4.7229 \\
\hline \multirow[t]{4}{*}{ BSA } & Humic & $16.0000^{*}$ & 1.96214 & .006 & 7.2771 & 24.7229 \\
\hline & Alginate $+\mathrm{Ca}$ & 4.5000 & 1.96214 & .047 & -4.2229 & 13.2229 \\
\hline & Alginate-Ca & $15.5000^{*}$ & 1.96214 & .007 & 6.7771 & 24.2229 \\
\hline & Mixture & $12.0000^{*}$ & 1.96214 & .017 & 3.2771 & 20.7229 \\
\hline \multirow[t]{4}{*}{ Alginate $+\mathrm{Ca}$} & Humic & $11.5000^{*}$ & 1.96214 & .019 & 2.7771 & 20.2229 \\
\hline & BSA & -4.5000 & 1.96214 & .047 & -13.2229 & 4.2229 \\
\hline & Alginate-Ca & $11.0000^{*}$ & 1.96214 & .023 & 2.2771 & 19.7229 \\
\hline & Mixture & 7.5000 & 1.96214 & .031 & -1.2229 & 16.2229 \\
\hline \multirow[t]{4}{*}{ Alginate-Ca } & Humic & .5000 & 1.96214 & .039 & -8.2229 & 9.2229 \\
\hline & BSA & $-15.5000^{*}$ & 1.96214 & .007 & -24.2229 & -6.7771 \\
\hline & Alginate $+\mathrm{Ca}$ & $-11.0000^{*}$ & 1.96214 & .023 & -19.7229 & -2.2771 \\
\hline & Mixture & -3.5000 & 1.96214 & .048 & -12.2229 & 5.2229 \\
\hline \multirow[t]{4}{*}{ Mixture } & Humic & 4.0000 & 1.96214 & .046 & -4.7229 & 12.7229 \\
\hline & BSA & $-12.0000^{*}$ & 1.96214 & .017 & -20.7229 & -3.2771 \\
\hline & Alginate $+\mathrm{Ca}$ & -7.5000 & 1.96214 & .031 & -16.2229 & 1.2229 \\
\hline & Alginate-Ca & 3.5000 & 1.96214 & .048 & -5.2229 & 12.2229 \\
\hline
\end{tabular}

*. The mean difference is significant at the .05 level. 